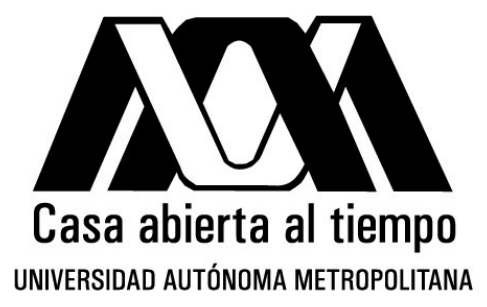

Universidad Autónoma Metropolitana - Unidad Iztapalapa

División de Ciencias Básicas e Ingeniería

Departamento de Matemáticas

\title{
Estabilidad De Equilibrios Relativos en Espacios Curvados
}

Tesis para obtener el grado de

Maestro en Ciencias Matemáticas

presenta

Juan Manuel Sánchez Cerritos

bajo la dirección de

Dr. Ernesto Pérez Chavela

México DF.

Diciembre de 2013 
A mis papás y hermanos, como testimonio de cariño y eterno agradecimiento. 


\section{Agradecimientos}

Agradezco de manera sincera al Profesor Ernesto Pérez Chavela por haber aceptado la dirección de esta tesis de maestría. Su orientación, apoyo y confianza en mí han sido un aporte invaluable para el desarrollo de este trabajo. Le agradezco también el haberme facilitado los medios suficientes para llevar a cabo las actividades académicas que mejoran mi formación.

Gracias a los profesores: Dr. Florin Diacu, Dr. Eduardo Piña Garza y Dr. Manuel Jesús Falconi Magaña por sus valiosos comentarios y sugerencias que ayudaron a mejorar el presente trabajo. Les agradezco por todo su tiempo invertido en la revisión de esta tesis.

A mis papás José Cruz y Gloria Felisa, y a mis hermanos Gloria Andrea y José Cruz, por todo su apoyo incondicional que me proporcionan día a día, además de su confianza depositada en mí.

Quiero agradecer a mi tía Lupe, a mi prima Heidy, a mis tíos Edgar y Martha, a mi abuelito Juan y a mis primos Edgar y Marycruz por haber ayudado a sentar las bases para el logro de este éxito.

Gracias a mis compañeros y amigos por haber influido en mi vida y el haber compartido conmigo sus conocimientos. En especial a Blanca por haber estado ahí en momentos fáciles y difíciles.

Agracezco al CONACYT por la beca otorgada a lo largo del programa de maestría. Número de becario 265709. 



\section{Índice general}

Dedicatoria

Agradecimientos

Índice general $\quad$ V

1. Introducción 1

2. Problema de $n$-cuerpos en espacios curvados $\quad 5$

2.1. Potencial Cotangente . . . . . . . . . . . . . . . . . . 7

2.2. Ecuaciones de Movimiento en $S_{k}^{2}$ y $H_{k}^{2} \ldots \ldots \ldots$. . . . . . . . . . 9

2.3. Forma Hamiltoniana . . . . . . . . . . . . . . . . . . . . . . . . . . 12

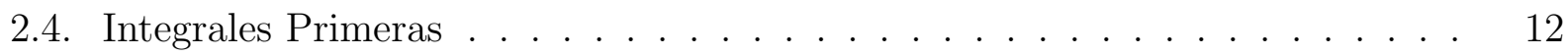

2.5. Singularidades . . . . . . . . . . . . . . . . . . 13

3. Problema de $n$-Cuerpos en $S^{2} \quad 15$

3.1. Equilibrios Relativos en $S^{2} \ldots \ldots \ldots$. . . . . . . . . . . . . . . . . . . . . . . . . .

3.2. Equilibrios Relativos Eulerianos . . . . . . . . . . . . . . . 28

4. Problema de $n$-Cuerpos en $H^{2} \quad 33$

4.1. Equilibrios Relativos en $H^{2}$. . . . . . . . . . . . . . . . . . . . . . . . . . . 33

4.1.1. Equilibrios Relativos Elípticos . . . . . . . . . . . . . . . . . 35

4.1.2. Equilibrios Relativos Hiperbólicos . . . . . . . . . . . . . . . . . . 38

4.1.3. Equilibrios Relativos Parabólicos . . . . . . . . . . . . . 41

5. Estabilidad de Equilibrios Relativos en $S^{2} \quad 43$

5.1. Problema de Dos Cuerpos . . . . . . . . . . . . . . . . . 43

5.2. Problema de Tres Cuerpos . . . . . . . . . . . . . . . 51

5.2.1. Problema Restringido . . . . . . . . . . . . . . . 52

5.2.2. Masas Iguales . . . . . . . . . . . . . . . . . . . . . . 64

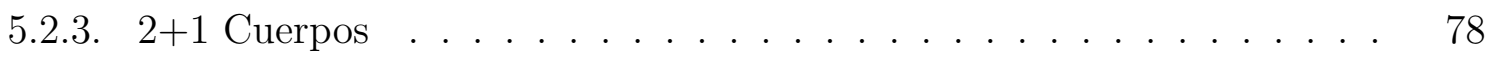


6. Estabilidad de Equilibrios Relativos en $H^{2} \quad 85$

6.1. Equilibrios Relativos Elípticos . . . . . . . . . . . . . . . . 85

6.1.1. Problema de Dos Cuerpos . . . . . . . . . . . . . . . 85

6.1.2. Problema de Tres Cuerpos . . . . . . . . . . . . . . . . . 89

6.1.2.1. Problema Restringido . . . . . . . . . . . . . . . . 89

6.1.2.2. Masas Iguales . . . . . . . . . . . . . . . . . . . . . 96

6.1.2.3. $2+1$ Cuerpos . . . . . . . . . . . . . . . 104

6.2. Equilibrios Relativos Hiperbólicos . . . . . . . . . . . . . . . . . . . . 108

6.2.1. Problema de Dos Cuerpos . . . . . . . . . . . . . . . . 108

6.2.2. Problema de Tres Cuerpos . . . . . . . . . . . . . . . . . . 115

6.2.2.1. Problema Restringido . . . . . . . . . . . . . . 116

$\begin{array}{lr}\text { 7. Conclusiones } & 129\end{array}$

$\begin{array}{ll}\text { Apéndice } & 133\end{array}$

$\begin{array}{ll}\text { Bibliografía } & 137\end{array}$

$\begin{array}{lr}\text { Índice alfabético } & 140\end{array}$ 


\section{Capítulo 1 Introducción}

Las observaciones hechas desde la antiguedad al movimiento de los planetas han hecho que algunos autores como [29,33], consideren a la astronomía como la madre de las ciencias, y le corresponde a la mecánica celeste describir la dinámica planetaria que rige no solo nuestro sistema solar, sino todo el universo.

Algunos de los resultados matemáticos más sobresalientes de la mecánica celeste se remontan al siglo XVII cuando Newton da una formulación matemática de las observaciones que Galileo y Kepler habían realizado anteriormente, sus resultados también fueron gracias a la invención del cálculo y al descubrimiento de la ley de la gravitación universal.

En general la mecánica celeste estudia el problema de los $n$-cuerpos, y aunque nace con Isaac Newton en la famosa obra Philosophiae Naturalis Principia Mathematica, [28], es con Henri Poincaré con quien adquiere su forma actual a finales del siglo XIX con su publicación On the Three-Body Problem and the Equations of Dynamics en [31], este trabajo presenta la solución de un problema propuesto por Karl Weierstrass que intentaba explicar el problema general de los $n$-cuerpos y del cual Poincaré explicó que la evolución de un sistema general era extremadamente caótica, por lo que aplicó sus conocimientos para estudiar el caso partícular con $n=3$, el problema restringido, que es aquel en donde se tiene un sistema de 3 partículas puntuales, pero que una de ellas es de masa pequeña, de tal forma que la fuerza que ejerce sobre las otras dos partículas es despreciable.

El problema de los dos cuerpos, o problema de Kepler, es el más sencillo de la mecánica celeste, las soluciones de este problema se conocen completamente. Newton demostró que las leyes de movimiento de Kepler corresponden al problema de dos cuerpos y proporcionó una solución geométrica de este problema para dos esferas moviéndose bajo su atracción gravitacional mutua [28]. Este problema, aunque es considerado como el más sencillo, en los últimos años también ha sido objeto de estudio profundo para analizar diferentes tipos de perturbaciones y así tratar de modelar problemas más complejos. Un ejemplo de ésto es el modelar el sistema Tierra-Sol-Luna, que puede considerarse como un problema restringido, Tierra y Sol, y una perturbación que es la fuerza de gravedad ejercida por la Luna. 
Una vez que se entendió por completo el problema de los dos cuerpos, los investigadores siguieron, naturalmente, con el problema de los tres cuerpos. El problema de los tres cuerpos consiste en determinar las posiciones y las velocidades, en cualquier instante, de tres masas puntuales sometidas a su atracción gravitacional mutua y partiendo de posiciones y velocidades dadas (condiciones iniciales). Este problema claramente no surge como un problema teórico, el sistema Tierra-Sol-Luna puede ser considerado como un caso próximo a éste. Charles Delaunay estudió dicho problema, entre los años 1860 y 1867, mostrando en sus trabajos que se trataba de un sistema inestable. Así, Delaunay y Poincaré fueron los primeros en introducir a la mecánica celeste, en sus trabajos del problema de los tres cuerpos, el término del caos, es decir, mostraron que pequeñas variaciones en las condiciones iniciales llevaban a resultados completamente diferentes.

El problema de los tres cuerpos es tan complejo que hasta el día de hoy sigue siendo materia de estudio profundo. Cabe señalar que las únicas soluciones explícitas que se conocen son las llamadas soluciones homográficas. Algunas soluciones muy especiales en el problema newtoniano son aquellas que se generan a partir de configuraciones centrales (caso partícular de las soluciones homográficas) son los llamados equilibrios relativos, estas son soluciones del sistema en donde de los cuerpos se encuentran moviéndose como cuerpo rígido, definiremos este concepto más detalladamente.

Como hemos mencionado, la mecánica celeste surge como una rama de las matemáticas en donde el hombre trata de entender el universo en el que vive, sin embargo hay autores que se han aventurado a estudiar casos más complejos que surgen de los problemas clásicos de la mecánica celeste, tal es el caso de Nikolai Lovachevsky, quien en 1835 propuso un problema de Kepler en el espacio hiperbólico de tres dimensiones [23], independientemente a Lovachevsky, Janos Bolyai propuso una idea similar, [2].

En 1860, Paul Joseph Serret propuso extender la fuerza gravitacional a la esfera $S^{2}$ y resolvió en ese espacio el correspondiente problema de Kepler, [35]. Otro gran matemático que se interesó por este nuevo problema fue Rudolph Lipschitz, quien definió un potencial proporcional a $1 / \sin \left(\frac{r}{R}\right)$, donde $r$ es la distancia entre los cuerpos y $R$ es el radio de curvatura de la esfera, [22].

En 1870, Ernst Schering revisó el trabajo de Lovachevsky y obtuvo expresiones analíticas dados por el potencial cotangente, mismo que mostraremos en este trabajo.

En 1885, Wilhelm Killing tomó la idea de Lovachevsky y la llevó al espacio $S^{3}$ y definió una extensión del potencial Newtoniano dado por la inversa del área de una esfera de dimensión 2, logrando una generalización de las tres leyes de Kepler, [18]. Otro gran investigador interesado en este problema fue el alemán Heinrich Liebmann quien mostró que las órbitas en el problema de los dos cuerpos en $S^{3}$ son cónicas, [21].

Otras aplicaciones a las que dio lugar este interesante problema se han dado en el área 
de física cuántica, Erwin Schroedinger, en 1940, usó en sus trabajos de mecánica cuántica el mismo potencial cotangente de Schering y Liebmann, [34].

Algunos trabajos más recientes son los de Cariñena, Rañada y Santander, [4], quienes probaron que las órbitas cónicas conocidas en el espacio Euclidiano se extienden naturalmente en espacios de curvatura constante, los cuales concuerdan con los resultados obtenidos por Liebmann.

Las ecuaciones de movimiento para el problema curvado de $\operatorname{los} n$-cuerpos $(n>2)$ en espacios de curvatura constante fueron obtenidas por Florin Diacu, Ernesto Pérez Chavela y Manuel Santoprete [10, 11], usando el potencial cotangente que mencionamos anteriormente. En esos trabajos se analizan soluciones de equilibrios relativos de tipo eulerianos y lagrangianos, mostrando condiciones para la existencia de éstos en espacios de curvatura constante, además se estudian ciertos tipos de colisiones.

El objetivo de este trabajo de tesis es estudiar, entender y lograr explicar los resultados que se tienen acerca del problema curvado de tres cuerpos usando el potencial cotangente, en especial acerca de los resultados que se tienen sobre los equilibrios relativos. También nos pusimos como objetivo obtener resultados de forma análitica sobre condiciones de estabilidad espectral de equilibrios relativos del tipo euleriano del problema de dos y tres cuerpos en espacios curvados. A pesar de las dificultades que se presentaron logramos obtener resultados originales.

Veremos que estudiar el problema en espacios de curvatura constante es lo mismo que analizar el problema de $n$-cuerpos en una esfera (para curvatura positiva) o en un hiperboloide (para curvatura negativa), ver figuras (2.1) y (2.2). El problema, expuesto en estos espacios, aparenta ser una cuestión no tan complicada, sin embargo termina siendo una tarea difícil ya que tuvimos que analizar matrices cuyas entradas son funciones en variables dadas por las masas y por las posiciones de las partículas, además de estudiar también sus respectivos polinomios característicos lo que implica una variable más en el análisis. La complejidad surge porque se estudian polinomios característicos de grado 12 en la variable $\mu$, y los coeficientes de estos polinomios son funciones racionales en variables de las masas, $m$, y en variable de la altura (posición), z, que llegan a ser de grado 6 y 9 , respectivamente.

En el capítulo 2 mostramos el planteamiento del problema de los $n$-cuerpos en espacios curvados, exponemos el potencial cotangente, la formulación hamiltoniana del problema en estos espacios, además de las singularidades dadas por las ecuaciones de movimiento y las integrales primeras.

En el capítulo 3 y 4 exhibimos algunos resultados importantes que hay en la literatura acerca del problema en espacios de curvatura positiva y negativa, respectivamente, como son condiciones para la existencia de ciertos tipos de equilibrios relativos. 
Todos los resultados que exponemos en el capítulo 5 son originales, mostramos regiones en donde los equilibrios relativos del problema de dos cuerpos son estables e inestables; sobre el problema de tres cuerpos cabe señalar que junto con los trabajos de Martínez y Simó [25] dan una idea clara del movimiento que generan los equilibrios relativos del problema de tres cuerpos en espacios de curvatura positiva. Ellos exponen regiones de estabilidad, en términos del momento angular, de los equilibrios relativos del tipo lagrangiano, y nosotros mostramos regiones de estabilidad en términos de la posición de las partículas en los equilibrios relativos del tipo euleriano.

Además en el capítulo 6 mostramos nuestros resultados sobre estabilidad de los equilibrios relativos eulerianos, del problema de dos y tres cuerpos, en espacios de curvatura negativa mismos que también son resultados originales ya que no existen trabajos realizados sobre estabilidad en estos espacios.

Terminamos el trabajo con el capítulo 7 resumiendo nuestros resultados en las conclusiones. 


\section{Capítulo 2 \\ Problema de $n$-cuerpos en espacios curvados}

En esta sección mostraremos notación, algunos conceptos y resultados para introducir el problema de los $n$-cuerpos en espacios de curvatura constante.

Consideremos la superficie generada por una función $f: \mathbb{R}^{2} \rightarrow \mathbb{R}$. Existen diferentes maneras de definir la curvatura de dicha superficie, cada una enfocándose en un aspecto diferente de la curvatura.

Definición 2.0.1 Diremos que una superficie dada por la ecuación $F(x, y, z)=0$ es no singular en el punto $P=\left(x_{0}, y_{0}, z_{0}\right)$ si el gradiente de $F$ en el punto $P$ es diferente de cero, es decir

$$
\frac{\partial F}{\partial x} e_{1}+\frac{\partial F}{\partial y} e_{2}+\frac{\partial F}{\partial z} e_{3} \neq 0
$$

en $x=x_{0}, y=y_{0}, z=z_{0}$.

Supongamos que tenemos una superficie en el espacio euclideano de dimensión 3 y un punto no singular $\left(x_{0}, y_{0}, z_{0}\right)$ en ella. Entonces la superficie alrededor del punto $\left(x_{0}, y_{0}, z_{0}\right)$ puede ser escrita, bajo una rotación adecuada, por una ecuación de la forma $z=f(x, y)$, donde $z_{0}=f\left(x_{0} \cdot y_{0}\right)$, y además

$$
\frac{\partial f}{\partial x_{x=x_{0}, y=y_{0}}}={\frac{\partial f}{\partial y_{x=x_{0}, y=y_{0}}}}=0 .
$$

Ahora consideamos la segunda diferencial de la función $z=f(x, y)$ (la cual suponemos que tiene segundas derivadas continuas), es decir $d^{2} f=f_{x x} d x^{2}+2 f_{x y} d x d y+f_{y y} d y^{2}$. La matriz de esta forma cuadrática, la denotaremos por $\left(a_{i j}\right)$, donde $a_{i j}=f_{x^{i} x^{j}}$, con $x^{1}=x, x^{2}=y$.

Definición 2.0.2 Sea una superficie $z=f(x, y)$, y un punto $P=\left(x_{0}, y_{0}, z_{0}\right)$ en ella donde $\nabla f=0$.

- Definimos la curvatura principal de la superficie en $P$ como los eigenvalores de la matriz $\left(a_{i j}\right)$ evaluada en $P$. 
- Llamamos la curvatura gaussiana de la superficie en $P$ al $\operatorname{det}\left(a_{i j}\right)$ de la matriz evaluada en $P$.

- También llamamos curvatura media de la superficie en $P$ a la traza de $\left(a_{i j}\right)$ evaluada en $P$.

De esta manera si llamamos $k_{1}, k_{2}$ a los eigenvalores de $a_{i j}$ evaluada en $x_{0}, y_{0}, z_{0}$, tenemos que $k_{1}$ y $k_{2}$ son las curvaturas principales, mientras que la curvatura gaussiana es $k=k_{1} k_{2}=a_{11} a_{22}-a_{12} a_{21}$, y la curvatura media es $a_{11}+a_{22}=k_{1}+k_{2}$. Se puede ver que la curvatura gaussiana es un invariante intrínseco de la superficie, es decir la curvatura gaussiana depende solo de las propiedades de la métrica de la superficie, tal resultado es conocido como el Teorema de Gauss [13].

Consideremos el problema de los $n$-cuerpos moviéndose sobre una superficie con curvatura gaussiana constante $k \neq 0$. Denotemos las masas de los $n$ cuerpos como $m_{1}, m_{2}, \cdots, m_{n}$. El siguiente teorema permite restringir el problema a una esfera, si la curvatura gaussiana es positiva, o a la hoja superior de un hiperboloide, si la curvatura es negativa (dotado de la métrica de Minkowski, ver apéndice), figuras (2.1) y (2.2). El siguiente es un teorema importante de geométria diferencial cuya demostración la podemos ver en [37].

Teorema 2.0.1 Dos superficies que tienen la misma curvatura Gaussiana constante son localmente isométricos.

Más adelante mostraremos que nuestro análisis lo podemos restringir a $S^{2}$ y a $H^{2}(k=1$ y $k=-1$ ), dotado con la métrica de Minkowski (ver apéndice).

Existen muchos ejemplos de superficies de curvatura constante, quizá los más sencillos son la esfera para curvatura positiva y una de las hojas del hiperboloide (dotado de la métrica de Minkowski, ver apéndice) para curvatura negativa.

En el caso de curvatura positiva, la esfera tiene curvatura constante $k=1 / R^{2}$, donde $R$ es el radio de la esfera. Dicha esfera está dada por la ecuación $x^{2}+y^{2}+z^{2}=k^{-1}$, (la denotaremos como $S_{k}^{2}$ ). En el caso negativo el hiperboloide de dos hojas (dotado con la métrica de Minkowski, ver apéndice) tiene curvatura $k=-1 / R^{2}$, cuya ecuación es $x^{2}+y^{2}-z^{2}=k^{-1}$, esta superficie la denotaremos por $H_{k}^{2}$. Para $k=0$ recuperamos el plano euclideano. Las coordenadas de posición del cuerpo $m_{i}$ están dadas por $q_{i}=\left(x_{i}, y_{i}, z_{i}\right)$ y una constante que depende del valor de $k$ que restringe el movimiento de la partícula a la superficie curvada.

\section{Sobre la trigonometría de las superficies}

Como se hace en [4] podemos generalizar las funciones trigonométricas $\sin _{k} \mathrm{y} \cos _{k}$ en espacios de curvatura constante $k$ de la siguiente forma 
Para $k>0$

$$
\begin{array}{r}
\sin _{k}(x)=k^{-1 / 2} \sin k^{1 / 2} x, \\
\cos _{k}(x)=\cos k^{1 / 2} x .
\end{array}
$$

para $k<0$

$$
\begin{array}{r}
\sin _{k}(x)=(-k)^{-1 / 2} \sinh (-k)^{1 / 2} x, \\
\cos _{k}(x)=\cosh (-k)^{1 / 2} x .
\end{array}
$$

De la misma forma que en el plano ecuideano definimos para $k>0$ y $k<0$ las funciones tangente y cotangente

$$
\tan _{k}=\frac{\sin _{k}(x)}{\cos _{k}(x)}, \quad \cot _{k}=\frac{\cos _{k}(x)}{\sin _{k}(x)} .
$$

Podemos también definir las respectivas funciones inversas, por ejemplo

$$
\begin{array}{r}
\cos _{k}^{-1}(y)=k^{1 / 2} \cos ^{-1}(y), \quad k>0 \\
\cos _{k}^{-1}(y)=(-k)^{1 / 2} \cos ^{-1}(y), \quad k<0 .
\end{array}
$$

\subsection{Potencial Cotangente}

Empecemos con un poco de notación. Sea $\widehat{\nabla}_{q_{i}}=\left(\partial_{x_{i}}, \partial_{y_{i}}, \sigma \partial_{z_{i}}\right)$ el gradiente respecto a $q_{i}$, donde $\sigma=1$ para $k \geq 0$ y $\sigma=-1$ para $k<0$. Usaremos $\nabla_{q_{i}}$ para denotar el gradiente con $k>0$, y $\bar{\nabla}_{q_{i}}$ para $k<0$. Denotemos también $\widehat{\nabla}=\left(\widehat{\nabla}_{q_{1}}, \widehat{\nabla}_{q_{2}}, \cdots, \widehat{\nabla}_{q_{n}}\right)$. Definamos el siguiente producto cruz y producto punto, dados $a=\left(a_{x}, a_{y}, a_{z}\right)$ y $b=\left(b_{x}, b_{y}, b_{z}\right)$

$$
\begin{array}{r}
a * b=\left(a_{x} b_{x}+a_{y} b_{y}+\sigma a_{z} b_{z}\right) \\
a \otimes b=\left(a_{y} b_{z}-a_{z} b_{y}, a_{z} b_{x}-a_{x} b_{z}, \sigma\left(a_{x} b_{y}-a_{y} b_{x}\right)\right) .
\end{array}
$$

Definamos la distancia entre dos puntos como sigue

$$
d_{k}(a, b)=\left\{\begin{array}{cc}
|k|^{-1 / 2} \cos _{k}^{-1}(k a * b) & k \neq 0 \\
|a-b| & k=0,
\end{array}\right.
$$

donde $|\cdot|$ es la norma euclidiana.

Además podemos hacer un reescalamiento en las unidades de las masas de tal forma que la constante de gravitación $G$ sea igual a 1. Definimos entonces el potencial cotangente del problema de $n$-cuerpos como la función $-U_{k}(q)$ donde 


$$
U_{k}=\frac{1}{2} \sum_{i=1}^{n} \sum_{j=1, i \neq j}^{n} m_{i} m_{j} \cot _{k}\left(d_{k}\left(q_{i}, q_{j}\right)\right),
$$

es la función de fuerza y $q=\left(q_{1}, q_{2}, \cdots, q_{n}\right)$ es la configuración del sistema. Si tomamos $k \neq 0$ obtenemos

$$
U_{k}(q)=\frac{1}{2} \sum_{i=1}^{n} \sum_{j=1, j \neq i}^{n} \frac{m_{i} m_{j}(\sigma k)^{1 / 2} k q_{i} * q_{j}}{\sqrt{\sigma-\sigma\left(k q_{i} * q_{j}\right)^{2}}} .
$$

Este potencial no es el único que extiende el potencial newtoniano a espacios de curvatura constante, sin embargo usaremos este potencial a lo largo del trabajo ya que como podemos ver en el trabajo de Kozlov [19] es el único potencial que extiende el potencial newtoniano que satisface que es una función armónica y que genera un flujo en donde todas las órbitas acotadas son cerradas (Teorema de Betrand).

Notemos que la función potencial satisface $U_{k}(\eta q)=U_{k}(q)=\eta^{0} U_{k}(q)$ para $\eta \neq 0$, lo que significa que el potencial es una función homogénea de grado cero.

Usaremos la fórmula de Euler para funciones homogéneas que nos servirá para deducir las ecuaciones de movimiento.

Enunciaremos la fórmula de Euler a través del siguiente

Teorema 2.1.1 $F: \mathbb{R}^{3 n} \rightarrow \mathbb{R}$ es una función diferenciable homogenea de grado $\eta$, si y solo si para cada $x \in \mathbb{R}^{3 n}$

$$
\eta F(x)=x * \widehat{\nabla} F(x)
$$

Como el potencial $U_{k}$ es una función homogénea de grado 0, entonces en nuestra notación

$$
q * \widehat{\nabla} U_{k}(q)=0
$$

Podemos escribir también a la función $U_{k}(q)=\frac{1}{2} \sum_{i=1}^{n} U_{k}^{i}\left(q_{i}\right)$, donde

$$
U_{k}^{i}\left(q_{i}\right)=\sum_{j=1, j \neq i}^{n} \frac{m_{i} m_{j}(\sigma k)^{1 / 2} k q_{i} * q_{j}}{\sqrt{\sigma-\sigma\left(k q_{i} * q_{j}\right)^{2}}}, \quad i=1, \cdots, n,
$$

también son funciones homogéneas de grado 0. Si aplicamos a estas funciones la fórmula de Euler, obtenemos $q_{i} * \widehat{\nabla}_{q_{i}} U_{k}^{i}(q)=0$. Además usando la identidad $\widehat{\nabla}_{q_{i}} U_{k}(q)=\widehat{\nabla}_{q_{i}} U_{k}^{i}\left(q_{i}\right)$, obtenemos

$$
q_{i} * \widehat{\nabla}_{q_{i}} U_{k}(q)=0, \quad i=1, \cdots, n .
$$




\subsection{Ecuaciones de Movimiento en $S_{k}^{2}$ y $H_{k}^{2}$}

Para obtener las euaciones de movimiento en $S_{k}^{2}$ y $H_{k}^{2}$ aplicamos un método variacional al la función (2.14). Notemos que el Lagrangiano tiene la forma

$$
L_{k}(q, \dot{q})=T_{k}(q, \dot{q})+U_{k}(q),
$$

donde $T_{k}(q, \dot{q})=\frac{1}{2} \sum_{i=1}^{n} m_{i}\left(\dot{q}_{i} * \dot{q}_{i}\right)\left(k q_{i} * q_{i}\right)$ es la energía cinética del sistema. Las ecuaciones de Euler-Lagrange están dadas por

$$
\frac{d}{d t}\left(\frac{\partial L_{k}}{\partial \dot{q}_{i}}\right)-\frac{\partial L_{k}}{\partial q_{i}}-\lambda_{k}^{i}(t) \frac{\partial f_{k}^{i}}{\partial q_{i}}=0, \quad i=1, \cdots, n,
$$

donde $f_{k}^{i}=q_{i} * q_{i}-k^{-1}$ es la restricción que mantiene a la partícula $i$ sobre la superficie de curvatura constante $k$, y $\lambda_{k}^{i}$ es el multiplicador de Lagrange correspondiente al cuerpo $m_{i}$. Tenemos $\dot{q}_{i} * q_{i}=0$, por lo que

$$
\frac{d}{d t}\left(\frac{\partial L_{k}}{\partial \dot{q}_{i}}\right)=m_{i} \ddot{q}_{i}\left(k q_{i} * q_{i}\right)+2 m_{i}\left(k \dot{q}_{i} * q_{i}\right)=m_{i} \ddot{q}_{i} .
$$

Esta ecuación junto con

$$
\frac{\partial L_{k}}{\partial q_{i}}=m_{i} k\left(\dot{q}_{i} * \dot{q}_{i}\right) q_{i}+\widehat{\nabla}_{q_{i}} U_{k}(q)
$$

implican que las ecuaciones (2.20) son equivalentes a

$$
m_{i} \ddot{q}_{i}-m_{i} k\left(\dot{q}_{i} * \dot{q}_{i}\right) q_{i}-\widehat{\nabla}_{q_{i}} k(q)-2 \lambda_{k}^{i}(t) q_{i}=0, \quad i=1, \cdots, n .
$$

Para determinar $\lambda_{i}^{k}$ notemos que $0=\ddot{f}_{k}^{i}=2 \dot{q}_{i} * q_{i}+2\left(q_{i} * \ddot{q}_{i}\right)$, entonces

$$
q_{i} * \ddot{q}_{i}=-\dot{q}_{i} * \dot{q}_{i} .
$$

Notemos que si hacemos el producto punto de (2.23) por $q_{i}$ y usando la fórmula de Euler obtenemos

$$
m_{i}\left(q_{i} * q_{i}\right)-m_{i} k\left(\dot{q}_{i} * \dot{q}_{i}\right)-q_{i} * \widehat{\nabla}_{q_{i}} U_{k}(q)=2 \lambda_{k}^{i} q_{i} * q_{i}=2 k^{-1} \lambda_{k}^{i},
$$

que junto con (2.24) implican $\lambda_{k}^{i}=-k m_{i}\left(\dot{q}_{i} * \dot{q}_{i}\right)$. Sustituyendo estos valores de los multiplicadores de Lagrange en (2.23), las ecuaciones de movimiento y las restricciones quedan

$$
m_{i} \ddot{q}_{i}=\widehat{\nabla}_{q_{i}} U_{k}(q)-m_{i} k\left(\dot{q}_{i} * \dot{q}_{i}\right) q_{i}, \quad q_{i} * q_{i}=k^{-1}, \quad k \neq 0, \quad i=1, \cdots, n .
$$

La coordenada $q_{i}$ del gradiente $U$, obtenida de (2.14), tiene la forma

$$
\widehat{\nabla}_{q_{i}} U_{k}(q)=\sum_{j=1, j \neq i}^{n} \frac{m_{i} m_{j}(\sigma k)^{1 / 2}\left(\sigma k q_{j}-\sigma\left(k^{2} q_{i} * q_{j}\right) q_{i}\right)}{\left[\sigma-\sigma\left(k q_{i} * q_{j}\right)^{2}\right]^{3 / 2}}, k \neq 0 .
$$


Que podemos reescribir como

$$
\widehat{\nabla}_{q_{i}} U_{k}(q)=\sum_{j=1, j \neq i}^{n} \frac{m_{i} m_{j}(\sigma k)^{3 / 2}\left[q_{j}-\left(k q_{i} * q_{j}\right) q_{i}\right]}{\left[\sigma-\sigma\left(k q_{i} * q_{j}\right)^{2}\right]^{3 / 2}}, \quad k \neq 0 .
$$

A continuación veremos que podemos quitar la dependencia sobre $k$. Enfoquémonos en la ecuación (2.26), y consideremos el siguiente reescalamiento sobre el tiempo

$$
q_{i}=|k|^{-1 / 2} r_{i}, \quad i=1,2, \cdots, n, \quad d \tau=|k|^{3 / 4} d t .
$$

Denotemos por $r_{i}^{\prime}$ y $r_{i}^{\prime \prime}$ a la primera y segunda derivada de $r_{i}$ respecto a $\tau$. Entonces las ecuaciones de movimiento (2.26) toman la forma

$$
r_{i}^{\prime \prime}=\sum_{j \neq i}^{n} \frac{m_{j}\left[r_{j}-\sigma\left(r_{i} * r_{j}\right) r_{i}\right]}{\left[\sigma-\sigma\left(r_{i} * r_{j}\right)^{2}\right]^{3 / 2}}-\sigma\left(r_{i}^{\prime} * r_{i}^{\prime}\right) r_{i}, \quad i=1,2, \cdots n
$$

Tenemos las ecuaciones de movimiento para el sistema que no depende del parámetro $k$; recordemos que tenemos $\sigma=1$ para $k>0$ y $\sigma=-1$ para $k<0$. Además notemos que

$$
r_{i} * r_{j}=|k| q_{i} * q_{i}=|k| k^{-1}=\sigma .
$$

Lo que muestra las nuevas variables $r_{i} \in S^{2}$ para $k>0$ y $r_{i} \in H^{2}$ para $k<0, i=1,2, \cdots n$.

Entonces para estudiar la dinámica del problema de $n$ cuerpos en espacios de curvatura constante podemos restringirnos al estudio sobre $H^{2}$ o $S^{2}$, ver figuras (2.1) y (2.2).

Las ecuaciones de movimiento son

- Para $k>0$

$$
\ddot{q}_{i}=\sum_{j \neq i}^{n} \frac{m_{j}\left[q_{j}-\left(q_{i} \cdot q_{j}\right) q_{i}\right]}{\left[1-\left(q_{i} \cdot q_{j}\right)^{2}\right]^{3 / 2}}-\left(\dot{q}_{i} \cdot \dot{q}_{i}\right) q_{i}, \quad i=1,2, \cdots, n .
$$

- Para $k<0$

$$
\ddot{q}_{i}=\sum_{j \neq i}^{n} \frac{m_{j}\left[q_{j}+\left(q_{i} \odot q_{j}\right) q_{i}\right]}{\left[\left(q_{i} \odot q_{j}\right)^{2}-1\right]^{3 / 2}}+\left(\dot{q}_{i} \odot \dot{q}_{i}\right) q_{i}, \quad i=1,2, \cdots, n
$$

Donde $(\odot)$ denota el producto en el modelo de Minkowski, es decir si $a, b \in \mathbb{R}^{3}$, entonces $a \odot b=\left(a_{x}, a_{y}, a_{z}\right) \odot\left(b_{x}, b_{y}, b_{z}\right)=a_{x} b_{x}+a_{y} b_{y}-a_{z} b_{z}$. 


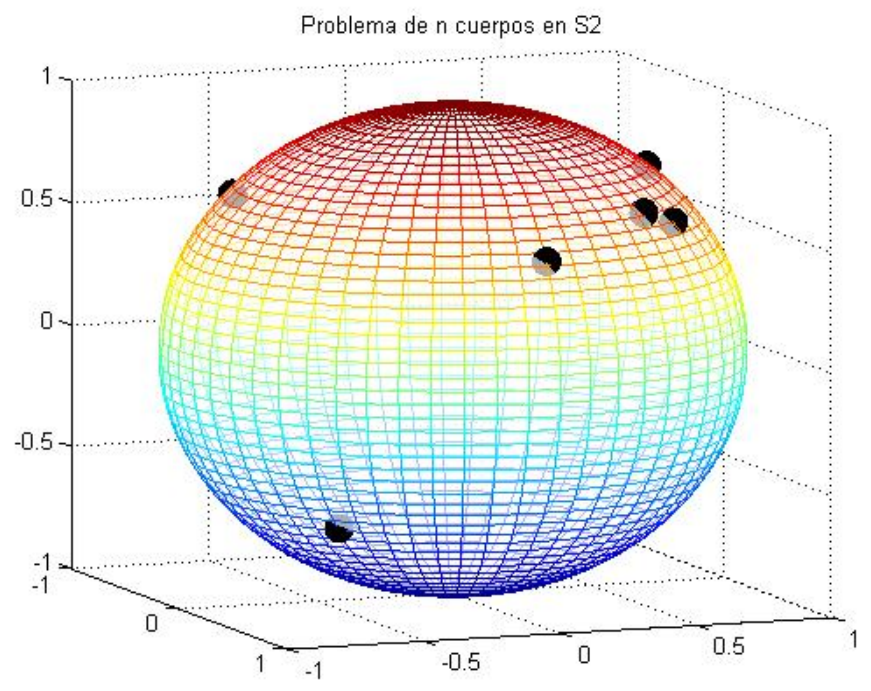

Figura 2.1: Problema de los $n$-cuerpos en $S^{2}$. Se pretende estudiar la iteracción de los cuerpos sujeta a la fuerza que generaliza el potencial newtoniano en el caso $n=3$.

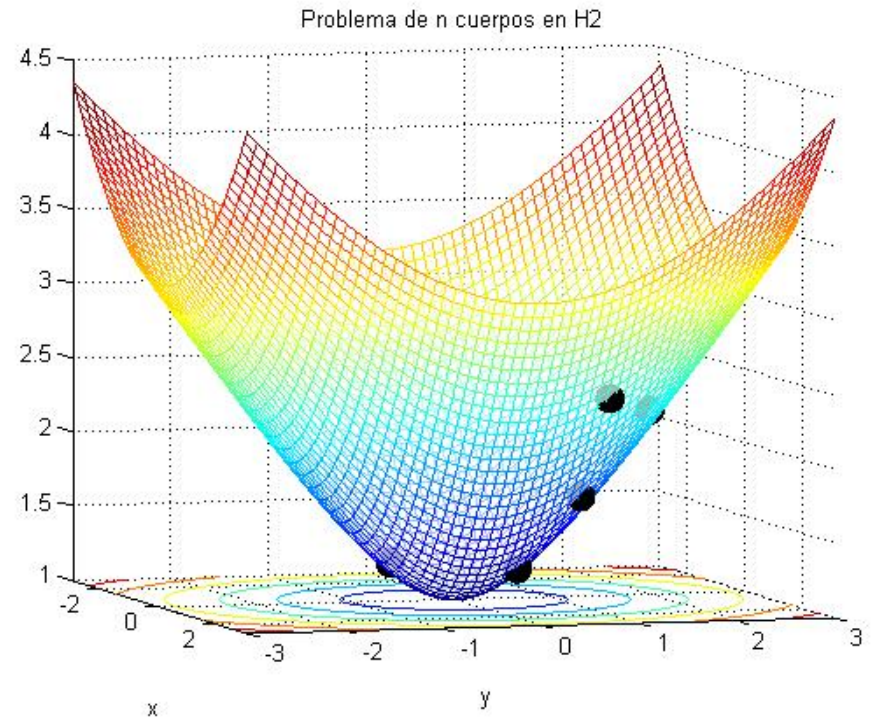

Figura 2.2: Problema de los $n$-cuerpos en $H^{2}$. Se pretende estudiar la iteracción de los cuerpos sujeta a la fuerza que generaliza el potencial newtoniano en el caso $n=3$. 


\subsection{Forma Hamiltoniana}

Las ecuaciones (2.26) generan un sistema Hamiltoniano como lo veremos a continuación. La función hamiltoniana $H_{k}$ está dada por

$$
H_{k}(q, p)=\frac{1}{2} \sum_{i=1}^{n} m_{i}^{-1}\left(p_{i} * p_{i}\right)\left(k q_{i} * q_{i}\right)-u_{k}(q), \quad q_{i} * q_{i}=k^{-1}, k \neq 0, i=1, \cdots n .
$$

Las ecuaciones (2.25) toman la forma del siguiente sistema hamiltoniano de dimensión $6 n$ con dos restricciones,

$$
\begin{aligned}
& \dot{q}_{i}=\widehat{\nabla}_{p_{i}} H_{k}(q, p)=m_{i}^{-1} p_{i}, \\
& \dot{p}_{i}=-\widehat{\nabla}_{q_{i}} H_{k}(q, p)=\widehat{\nabla}_{q_{i}} U_{k}(q)-m_{i}^{-1} k\left(p_{i} * p_{i}\right) q_{i},
\end{aligned}
$$

con

$$
q_{i} * q_{i}=k^{-1}, \quad q_{i} * p_{i}=0, \quad k \neq 0, \quad i=1, \cdots, n .
$$

Estas ecuaciones describen el movimiento de un sistema de $n$ partículas para cualquier $k \neq 0$.

\subsection{Integrales Primeras}

Primero recordemos lo que es una integral primera con la siguiente definición.

Definición 2.4.1 Una integral primera, constante de movimiento o cantidad conservada de un sistema de ecuaciones diferenciales

$$
\dot{x}=f(t, x), x \in \mathbb{R}^{n}, f: A \subset \mathbb{R}^{n} \rightarrow \mathbb{R}^{n},
$$

es una función $F(t, x)$ tal que es constante a lo largo de las curvas integrales o soluciones del sistema, es decir

$$
F(t, x(t))=\text { cte, para todo } t \in \mathbb{R},
$$

y para toda solución $x(t)$ del sistema de ecuaciones diferenciales.

Las ecuaciones de movimiento (2.26) tienen a la energía como integral primera

$$
h=T_{k}(q, p)-U_{k}(q),
$$

donde $T_{k}(q, p)=\frac{1}{2} \sum_{i=1}^{n} m_{i}^{-1}\left(p_{i} * p_{i}\right)\left(k q_{i} * q_{i}\right)$ es la energía cinética, y $p=\left(p_{1}, \cdots, p_{n}\right)$ denota el momento de el sistema, con $p_{i}=m_{i} \dot{q}_{i}$ representando el momento del cuerpo con masa $m_{i}, i=1, \cdots, n, \mathrm{y}$ h es una constante real.

Multiplicando escalarmente (2.26) por $\dot{q}_{i}$ obtenemos 


$$
\sum_{i=1}^{n} m_{i} \ddot{q}_{i} * \dot{q}_{i}=\left(\widehat{\nabla}_{q_{i}}\right) \cdot \dot{q}_{i}-\sum_{i=1}^{n} m_{i} k\left(\dot{q}_{i} * \dot{q}_{i}\right) q_{i} * \dot{q}_{i}=\frac{d}{d t} U_{k}(q(t)) .
$$

La ecuación (2.37) se sigue inmediatamente de integrar (2.38).

Otras integrales de movimiento son las que surgen del momento angular

$$
\sum_{i=1}^{n} q_{i} \otimes p_{i}=c, \quad c \in \mathbb{R}^{3} .
$$

La ecuación (2.39) surge de multiplicar vectorialmente cada ecuación de (2.26) por la respectiva $q_{i}$ y sumar las $n$ ecuaciones; al hacer estas operaciones obtenemos

$$
\begin{aligned}
\sum_{i=1}^{n} m_{i} \ddot{q}_{i} \otimes q_{i}= & \sum_{i=1}^{n} \sum_{j=1, j \neq i}^{n} \frac{m_{i} m_{j}(\sigma k)^{3 / 2} q_{i} \otimes q_{j}}{\left[\sigma-\sigma\left(k q_{i} * q_{j}\right)^{2}\right]^{3 / 2}}- \\
& \sum_{i=1}^{n}\left[\sum_{j=1, j \neq i}^{n} \frac{m_{i} m_{j}(\sigma k)^{3 / 2}\left(k q_{i} * q_{j}\right)}{\left[\sigma-\sigma\left(k q_{i} * q_{j}\right)^{2}\right]^{3 / 2}}-m_{i}(\sigma k)\left(\dot{q}_{i} * \dot{q}_{i}\right)\right] q_{i} \otimes q_{i}=0,
\end{aligned}
$$

obtenemos (2.39) de la antisimetría del producto vectorial y de que $q_{i} \otimes q_{i}=0$.

\subsection{Singularidades}

Las ecuaciones de movimiento (2.26) generan algunas restricciones sobre el movimiento de las partículas, en esta sección las explicamos con más detalles. Una de las restricciones es que las partículas están moviéndose sobre la superficie de curvatura gaussiana constante, es decir $(q, p) \in T^{*}\left(M_{k}^{2}\right)^{n}$, donde $M_{K}^{2}$ es la superficie de curvatura constante $k \neq 0$, y $T^{*}\left(M_{k}^{2}\right)^{n}$ es el haz tangente de $M_{k}^{2}$. Existe otra restricción sobre las partículas y se genera porque el gradiente (2.27) no está definido en el conjunto

$$
\left\{q \in\left(M_{k}^{2}\right)^{n} \mid\left(k q_{i} * q_{j}\right)^{2}=1\right\}=: \triangle_{i j},
$$

donde las funciones (2.14) y (2.27) tienden a infinito. Si definimos $\triangle:=\cup_{1 \leq i<j \leq n} \triangle_{i j}$, entonces $\triangle$ contiene a todas las singularidades de las ecuaciones de movimiento.

La condición $\left(k q_{i} * q_{i}\right)^{2}=1$ nos sugiere que consideremos los siguientes conjuntos $\triangle_{i j}^{+} \mathrm{y}$ $\Delta_{i j}^{-}$, donde $\Delta_{i j}^{+}=\left\{q \in\left(M_{k}^{2}\right)^{n} \mid k q_{i} * q_{j}=1\right\} \quad$ y $\triangle_{i j}^{-}=\left\{q \in\left(M_{k}^{2}\right)^{n} \mid k q_{i} * q_{j}=-1\right\}$.

Definimos $\Delta^{+}:=\cup_{1 \leq i<j \leq n} \triangle_{i j}^{+}$y $\Delta^{-}:=\cup_{1 \leq i<j \leq n} \triangle_{i j}^{-}$, entonces tenemos $\Delta=\Delta^{+} \cup \Delta^{-}$. Los elementos de $\Delta^{+}$corresponden a las colisiones para cualquier $k \neq 0$, mientras que los elementos de $\Delta^{-}$corresponden a las llamadas singularidades antipodales, que se dan cuando $k>0$. 
Las singularidades antipodales se dan cuando dos cuerpos se encuentran en los lados opuestos de un círculo máximo sobre una esfera. Para $k<0$ este tipo de singularidades nunca ocurren porque $k q_{i} * q_{j} \geq 1$.

Resumiendo podemos mencionar lo siguiente, las ecuaciones de movimiento no están definidas para configuraciones en donde existen colisiones en esferas e hiperboloides, así como configuraciones con cuerpos en posiciones antipodales es esferas con cualquier curvatura $k>0$. En ambos casos la fuerza gravitacional tiende a infinito. 


\section{Capítulo 3 Problema de $n$-Cuerpos en $S^{2}$}

En este capítulo mostraremos resultados importantes del movimiento del problema de $n$-cuerpos en $S^{2}$.

Empecemos con el movimiento de una partícula sobre la esfera con el siguiente

Teorema 3.0.1 Una partícula libre en $S^{2}$ se encuentra en reposo o se mueve a lo largo de círculos máximos en órbitas cerradas.

\section{Demostración}

Como no hay interacción gravitacional con otros cuerpos tenemos que la ecuación de movimiento es

$$
\ddot{q}=-(\dot{q} \cdot \dot{q}) q
$$

donde $q$ es el vector que describe la posición del cuerpo de masa $m$. Si $\dot{q}(0)=0$, entonces $\ddot{q}(0)=0$, entonces no hay fuerzas que actuen sobre $m$. Entonces el cuerpo estará en reposo en todo tiempo.

Si $\dot{q} \neq 0$, entonces $q$ y $\ddot{q}$ son colineales, pero en dirección opuesta. Entonces la suma entre $\ddot{q}(0)$ y $\dot{q}(0)$ jalan al cuerpo a lo largo de la geodésica correspondiente a la dirección de estos vectores.

Mostraremos que el movimiento es uniforme. Este hecho lo veremos a partir de la integral de momento angular

$$
c=(q \times \dot{q}) \cdot(q \times \dot{q})=(q \cdot q)(\dot{q} \cdot \dot{q}) \sin ^{2} \alpha,
$$

donde $c$ es la longitud del momento angular y $\alpha$ es el ángulo entre $q$ y $\dot{q}$. Como $q \cdot q=1$, podemos concluir de (3.2) que la velocidad del cuerpo es constante.

Iniciaremos el estudio donde tenemos más de una partícula con el siguiente lema, nos ayudará a analizar un resultado importante de soluciones que surgen de analizar las singularidades del problema de 3 cuerpos. 
Lema 3.0.1 Consideremos el problema de $n$ cuerpos en $S^{2}$, y supongamos que un cuerpo de masa $m$ se encuentra en reposo al tiempo $t_{0}$ sobre la geodésica $z=0$ dentro de la región con $x, y>0$. Entonces, si

(a) $\ddot{x}\left(t_{0}\right)>0$ y $\ddot{y}\left(t_{0}\right)<0$, la fuerza jala al cuerpo a lo largo de un círculo hacia el punto $(1,0)$.

(b) $\ddot{x}\left(t_{0}\right)<0$ y $\ddot{y}\left(t_{0}\right)>0$, la fuerza jala al cuerpo a lo largo del círculo hacia el punto $(0,1)$.

(c) $\ddot{x}\left(t_{0}\right) \leq 0$ y $\ddot{y}\left(t_{0}\right) \leq 0$, la fuerza jala al cuerpo a lo largo del círculo hacia el punto $(1,0)$, si $\ddot{y}\left(t_{0}\right) / \ddot{x}\left(t_{0}\right)>y\left(t_{0}\right) / x\left(t_{0}\right)$; hacia el punto $(0,1)$ si $\ddot{y}\left(t_{0}\right) / \ddot{x}\left(t_{0}\right)<y\left(t_{0}\right) / x\left(t_{0}\right)$, pero no hay fuerzas actuando sobre el cuerpo si ninguna de las desigualdades anteriores se cumple.

(d) $\ddot{x}\left(t_{0}\right)>0$ y $y\left(t_{0}\right)>0$, el movimiento no es posible.

\section{Demostración}

Por la ecuación (2.24), $x \ddot{x}+y \ddot{y}=-\left(\dot{x}^{2}+\dot{y}^{2}\right) \leq 0$, lo que significa que la fuerza que actúa sobre $m$ siempre se dirige a lo largo de la tangente en $m$ del círculo geodésico $z=0$ o dentro del medio plano que contiene a este círculo. Asumiendo que un sistema $x y$-coordinado está fijo en el origen del vector aceleración, este vector siempre se encontrará en el medio plano debajo de la linea pendiente $-x\left(t_{0}\right) / y\left(t_{0}\right)$. Probaremos los siguientes casos de manera separada.

(a) Si $\ddot{x}\left(t_{0}\right)>0$ y $\ddot{y}\left(t_{0}\right)<0$, la fuerza que actúa sobre $m$ está representada por un vector que se encuentra en la región dada por la intersección del cuarto cuadrante y el medio plano debajo de la linea de pendiente $-x\left(t_{0}\right) / y\left(t_{0}\right)$. Entonces la fuerza jala al cuerpo a lo largo del círculo en la dirección del punto $(1,0)$.

(b) Si $\ddot{x}\left(t_{0}\right)<0$ y $\ddot{y}\left(t_{0}\right)>0$, la fuerza que actúa sobre $m$ está representada por un vector que cae en la región dada por la intersección del segundo cuadrante y el medio plano que se encuentra debajo de la linea con pendiente $-x\left(t_{0}\right) / y\left(t_{0}\right)$, entonces la fuerza jala al cuerpo a lo largo del círculo en la dirección del punto $(1,0)$.

(c) Si $\ddot{x}\left(t_{0}\right) \leq 0$ y $\ddot{y}\left(t_{0}\right) \leq 0$, la fuerza que actúa sobre $m$ está representada por un vector que se encuentra en el tercer cuadrante. Entonces la dirección con la cual la fuerza actúa depende del lugar donde se encuentre el vector aceleración: $(i)$ debajo de la linea con pendiente $x\left(t_{0}\right) / y\left(t_{0}\right),(i i)$ por arriba de la linea con pendiente $x\left(t_{0}\right) / y\left(t_{0}\right)$, $(i i i)$ sobre la linea con pendiente $x\left(t_{0}\right) / y\left(t_{0}\right)$. El último caso incluye cuando la aceleración es cero.

En el caso $(i)$, el vector aceleración se encuentra en una linea cuya pendiente es mayor que $x\left(t_{0}\right) / y\left(t_{0}\right)$, es decir $\ddot{y}\left(t_{0}\right) / \ddot{x}\left(t_{0}\right)>y\left(t_{0}\right) x\left(t_{0}\right)$, entonces la fuerza jala al cuerpo $m$ hacia $(1,0)$. En el caso $(i i)$, el vector aceleración se encuentra en una linea de pendiente menor que $x\left(t_{0}\right) / y\left(t_{0}\right)$, es decir $\ddot{y}\left(t_{0}\right) / \ddot{x}\left(t_{0}\right)<y\left(t_{0}\right) x\left(t_{0}\right)$, entonces la fuerza jala al 
cuerpo $m$ hacia $(1,0)$. En el caso (iii), el vector aceleración se encuentra en la linea de pendiente $x\left(t_{0}\right) / y\left(t_{0}\right)$, es decir $\ddot{y}\left(t_{0}\right) / \ddot{x}\left(t_{0}\right)=y\left(t_{0}\right) x\left(t_{0}\right)$. Pero el último caso no puede ocurrir. Este hecho se sigue de las ecuaciones de movimiento, las cuales muestran que la aceleración es la diferencia entre el gradiente de la función de fuerza y un mútiplo del vector de posición. Pero de acuerdo a la fórmula de Euler para funciones homogéneas, y el hecho de que las velocidades son cero, estos vectores son ortogonales, entonces su diferencia puede tener la misma dirección de uno de ellos sólo si es cero. Este argumento vectorial concuerda con el hecho de energía cinética, que muestra que si $\dot{x}(t)=\dot{y}(t)=0$ y la aceleración tiene la misma dirección que el vector posición, entonces $m$ no se mueve, luego $\dot{x}(t)=\dot{y}(t)=0$, y así $\ddot{x}(t)=\ddot{y}(t)=0$, ninguna fuerza actúa sobre $m$, el cuerpo se mantiene fijo.

(d) Si $\ddot{x}\left(t_{0}\right)>0$ y $\ddot{y}\left(t_{0}\right)>0$, la fuerza que actúa sobre $m$ está representada por el vector que se encuentra en la región dada por la intersección entre el primer cuadrante y el medio plano que se encuentra debajo de la linea con pendiente $-x\left(t_{0}\right) / y\left(t_{0}\right)$. Pero esta región es vacia, así que no existe movimiento.

En el problema de dos cuerpos, los conjuntos $\Delta^{+}$y $\Delta^{-}$son disjuntos, ya que como sólo hay dos cuerpos, tenemos $k q_{1} * q_{2}$ es 1 o -1 , pero no puede tomar los dos valores al mismo tiempo. Sin embargo para mas de tres cuerpos estos conjuntos no son disjuntos. En el problema de tres cuerpos, la configuración en donde dos cuerpos están en colisión y el tercero se encuentra en el lado opuesto del correspondiente círculo máximo es lo que se conoce como la singularidad de colisión antipodal. A continuación mostramos un resultado importante acerca de esta clase de colisiones.

Teorema 3.0.2 Consideremos el problema de 3 cuerpos en $S^{2}$ en donde los cuerpos $m_{1}$ y $m_{2}$ tienen masas $M>0$ y el cuerpo $m_{3}$ tiene masa $m>0$. Existen valores de $m$ y $M$, asi como condiciones iniciales para las cuales, las soluciones terminan en tiempo finito en una singularidad de colisión antipodal. Otras elecciones de masas y condiciones iniciales generan soluciones en donde los cuerpos se repelen de la singularidad de colisión antipodal.

\section{Demostración}

Las singularidades de colisión antipodal se dan cuando los cuerpos con masas $M$ colisionan en un punto diametralmente opuesto al cuerpo con masa $m$ sobre un circulo a altura $z$.

Consideremos las siguientes condiciones iniciales 


$$
\begin{array}{lll}
x_{1}(0)=-x(0), & y_{1}(0)=y(0), & z_{1}(0)=0 \\
x_{2}(0)=x(0), & y_{2}(0)=y(0), & z_{2}(0)=0 \\
x_{3}(0)=0, & y_{3}(0)=-1, & z_{3}(0)=0
\end{array}
$$

así como velocidades iniciales iguales a cero, y además $0<x(t), y(t)<1$ son funciones donde $x(t)^{2}+y(t)^{2}=1$. Como todas las coordenadas son cero, tomaremos en cuenta solo las coordenadas $x, y$ para el movimiento de las partículas. la simetría de las condiciones iniciales implican que $m_{3}$ se mantiene fija en todo tiempo $t$, que el momento angular es cero, y que es suficiente ver que pasa con $m_{2}$ porque $m_{1}$ se mueve simetricamente respecto al eje $y$. Si sustituimos las condiciones iniciales en las ecuaciones de movimiento obtenemos

$$
\begin{aligned}
& \ddot{x}(0)=-\frac{y(0)}{x^{2}(0)}\left(\frac{M}{4 y^{2}(0)}-m\right), \\
& \ddot{y}(0)=\frac{1}{x(0)}\left(\frac{M}{4 y^{2}(0)}-m\right) .
\end{aligned}
$$

Tenemos varias situaciones

1. Si $M \geq 4 m$ se sigue que $\ddot{x}(0)<0$ y $\ddot{y}(0)>0$ para cualquier elección de posiciones iniciales con $0<x(0), y(0)<1$.

2. Si $M<4 m$ entonces hay condiciones iniciales para las cuales
a $\ddot{x}(0)<0$ y $\ddot{y}(0)>0$,
b $\ddot{x}(0)>0$ y $\ddot{y}(0)<0$,
c $\ddot{x}(0)=\ddot{y}(0)=0$.

En el caso 2(c), las soluciones son puntos fijos del sistema, que además se debe cumplir $M=2 m$ y $x(0)=y(0)=\sqrt{2} / 2$. Lo que estudiaremos para mostrar el teorema son los casos 1 y $2(b)$. En el primer caso tenemos que $m_{2}$ comienza a moverse desde reposo hasta llegar a colisión con $m_{1}$ en el punto $(0,1)$, pero el lugar en donde llegan a colisión también depende de las velocidades que afectan a las ecuaciones de movimiento. En el último caso $m_{2}$ se mueve alejándose de la colisión, y debemos ver de nuevo como las velocidades alteran la tendencia inicial. Escribamos las ecuaciones de movimiento para $m_{2}$, con masas arbitrarias $m$ y $M$, tras algunos calculos tenemos

$$
\begin{array}{r}
\left.\ddot{x}=-\frac{M}{4 x^{2} y}+\frac{m y}{x^{2}}-\left(\dot{x}^{2}\right)+\dot{y}^{2}\right) x, \\
\left.\ddot{y}=\frac{M}{4 x y^{2}}+\frac{m}{x}-\left(\dot{x}^{2}\right)+\dot{y}^{2}\right) y,
\end{array}
$$


y con integral de energía

$$
\dot{x}^{2}+\dot{y}^{2}=\frac{h}{M}-\frac{2 m y}{x}+\frac{M\left(2 y^{2}-1\right)}{2 x y} .
$$

Usando la última ecuación en (3.6) obtenemos

$$
\begin{array}{r}
\ddot{x}=\frac{4(M-2 m) x^{4}-2(M-2 m) x^{2}-M+4 m}{4 x^{2} y}-\frac{h}{M} x, \\
\ddot{y}=\frac{M+2(M-2 m) y^{2}-4(M-2 m) y^{4}}{4 x y^{2}}-\frac{h}{M} y .
\end{array}
$$

Nos enfocaremos en las primeras órbitas que enuncia el teorema.

1. Para probar la existencia de soluciones con singularidad de colisión antipodal, examinemos el caso $M=4 m$, lo cual lleva al sistema (3.8) a la forma

$$
\begin{aligned}
& \ddot{x}=\frac{m\left(2 x^{2}-1\right)}{y}-\frac{h}{4 m} x, \\
& \ddot{y}=\frac{m x\left(2 y^{2}+1\right)}{y^{2}}-\frac{h}{4 m} y .
\end{aligned}
$$

Para estos valores de masas tenemos

$$
\dot{x}^{2}+\dot{y}^{2}+\frac{2 m x}{y}=\frac{h}{4 m} .
$$

Calculamos el valor de $h$ usando las condiciones iniciales. Para condiciones iniciales $x(0), y(0)$ y $\dot{x}(0)=\dot{x}(0)=0$, tenemos $h=8 m^{2} x(0) / y(0)>0$.

Si $x \rightarrow 0$, entonces $y \rightarrow 1$, y las ecuaciones (3.8) implican que $\ddot{x}(t) \rightarrow-m<0 \mathrm{y}$ $\ddot{y}(t) \rightarrow-h / 4 m<0$. Tenemos el caso (c) del lema (3.0.1), entonces para determinar la dirección de movimiento de $m_{2}$ cuando se acerca al punto $(0,1)$, tenemos que tomar en cuenta el radio $\ddot{y} / \ddot{x}$, que tiende a $h / 4 m^{2}$ cuando $x \rightarrow 0$. Como $h=8 m^{2} x(0) / y(0)$, $\lim _{x \rightarrow 0}(\ddot{y} / \ddot{x})=2 x(0) / y(0)$. Entonces $2 x(0) / y(0)<x(0)$ para cualquier $x(0)$ y $y(0)$ con $0<x(0)<1 / \sqrt{3}$ y la correspondiente $y(0)>0$ dada por la restricción $x^{2}(0)+y^{2}(0)=1$. Pero la desigualdad $2 x(0) / y(0)<y(0) / x(0)$ es equivalente a $\ddot{y}\left(t_{0}\right) / \ddot{x}\left(t_{0}\right)<y\left(t_{0}\right) / x\left(t_{0}\right)$ en lema (3.0.1) inciso (c), que concuerda con que la fuerza jala la partícula $m_{2}$ al punto $(0,1)$. Entonces la velocidad y la fuerza actuan sobre $m_{2}$ y hacen que el cuerpo siga la misma curva hasta llegar a la posición de colisión antipodal. 
Se tiene también de (3.9) que la velocidad es positiva y finita en colisión. Como la distancia entre la posición inicial y $(0,1)$ es acotada, $m_{2}$ colisiona con $m_{1}$ en tiempo finito. Así la elección de masas con $M=4 m$, posiciones iniciales $x(0), y(0)$ con $0<x(0)<1 / \sqrt{3}$, el correspondiente valor de $y(0)$ y velocidades iniciales $\dot{x}(0)=\dot{y}(0)$, llevan a solucion de singularidad de colisión antipodal.

Ahora demostraremos la existencia del segundo tipo de órbitas mencionadas en el teorema.

2. Para demostrar la existencia de soluciones que se repelen de una singularidad de colisión antipodal de las ecuaciones de movimiento en tiempo positivo, tomemos $M=2 \mathrm{~m}$.

Las ecuaciones (3.8) toman la forma

$$
\begin{aligned}
& \ddot{x}=\frac{m}{2 x^{2} y}-\frac{h}{2 m} x, \\
& \ddot{y}=\frac{m}{2 x y^{2}}-\frac{h}{2 m} y,
\end{aligned}
$$

con integral de energía

$$
\dot{x}^{2}+\dot{y}^{2}+\frac{m}{x y}=\frac{h}{2 m}
$$

ecuación que implica $h>0$. Como vimos en el caso 2(c) de este teorema, la posición inicial $x(0)=y(0)=\sqrt{2} / 2$ corresponde a un punto fijo de las ecuaciones de movimiento con velocidades iniciales iguales a cer. Así tenemos que buscar la solución deseada para las condiciones iniciales $0<x(0)<\sqrt{2} / 2$ y la correspondiente $y(0)$. Escojamos cualquier condición inicial para posiciones dentro de este conjunto, pero que sean lo suficientemente cerca a colisión como deseemos, y velocidades iniciales iguales a cero. Para $x \rightarrow 0$, las ecuaciones (3.10) muestran que $\ddot{x}$ y $\ddot{y}$ tienen crecimiento positivo. Pero de acuerdo al inciso (d) del lema (3.0.1), tal situación es imposible, por lo que el movimiento no puede ser infinitesimalmente cercana a la correspondiente singularidad de colisión antipodal, la cual repele cualquier solución con $M=2 m$ y condiciones iniciales escogidas como describimos anteriormente.

\subsection{Equilibrios Relativos en $S^{2}$}

En esta sección mostraremos algunos resultados importantes que se tienen del problema de los $n$-cuerpos relacionados con puntos fijos y una clase partícular de equilibrios relativos, 
mismos que definiremos en esta sección.

Los equilibrios relativos en el problema newtoniano de los $n$-cuerpos son las órbitas más simples, éstas son soluciones en donde las partículas se mueven uniformemente como cuerpo rígido. Extenderemos este concepto al problema curvado de los $n$ cuerpos.

Un teorema importante de geometría diferencial es el teorema del eje principal, que nos dice que cualquier rotación en $\mathbb{R}^{3}$ es a lo largo de un eje fijo, y podemos, sin pérdida de generalidad, tomar al eje $z$, notemos que podemos tomar las rotaciones a lo largo de este eje también gracias a que el potencial $U$ es invariante bajo el grupo de isometrías $S O(3)$. Recordemos que estas rotaciones están representadas por los subgrupos uniparamétricos de $S O(3)$ dados por la matriz

$$
A(t)=\left(\begin{array}{ccc}
\cos t & \sin t & 0 \\
-\sin t & \cos t & 0 \\
0 & 0 & 1
\end{array}\right)
$$

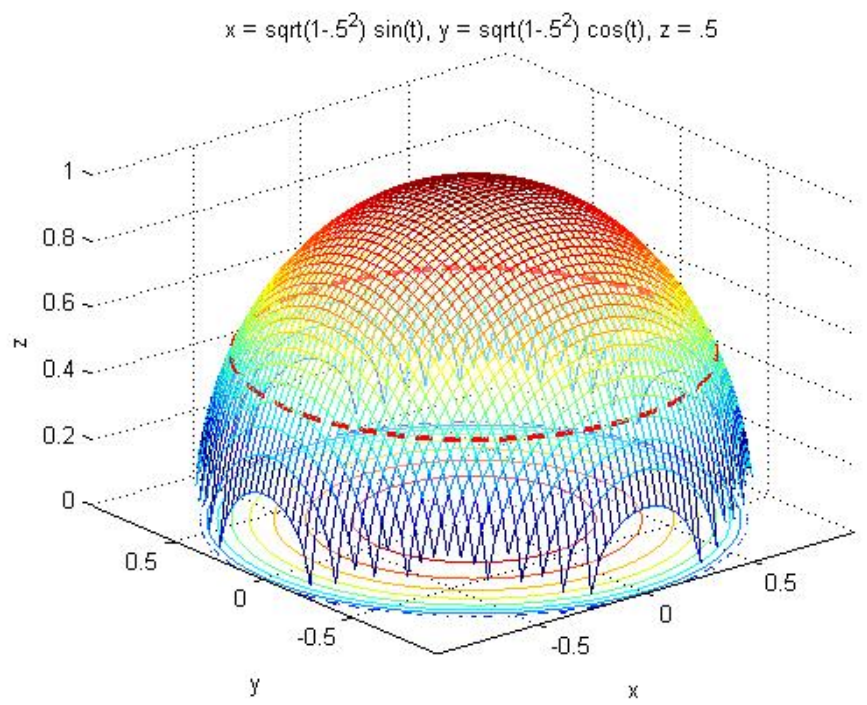

Figura 3.1: La linea punteada marca la trayectoria del punto $\left(\frac{1}{2}, \frac{1}{2}, \frac{1}{\sqrt{2}}\right)$ al aplicarle la transformación dada por la matriz $A(t)$.

Sea $S O(3)$ el grupo de isometrias de $S^{2}$, si denotamos por $\{G(t)\}$ al grupo uniparamétrico de $S O(3)$, entonces definimos a los equilibrios relativos de la siguiente forma

Definición 3.1.1 Un equilibrio relativo del problema de $n$-cuerpos en $S^{2}$ es una solución $q(t)$ de las ecuaciones de movimiento que es invariante bajo el subgrupo $\{G(t)\}$. En otras palabras, la función obtenida por la acción denotada por $w(t)=G(t) q(t)$ es también solución 
de las ecuaciones de movimiento. Dicho de otra forma, un equilibrio relativo en $S^{2}$ es una solución donde las partículas se mueven como cuerpo rígido, es decir sus distancias mutuas son invariantes durante todo el movimiento.

Un resultado de geometría diferencial nos muestra que el grupo propio de isometrias de $S^{2}$ es el cociente $S U(2) /\{ \pm I\}$ del subgrupo especial unitario

$$
S U(2)=\left\{A \in G L(2, \mathbb{C}) \mid A^{T} A=I\right),
$$

donde cada matriz $A$ tiene la forma

$$
A=\left(\begin{array}{cc}
a & b \\
-\bar{b} & \bar{a}
\end{array}\right)
$$

donde $a, b \in \mathbb{C}$ satisfacen $|a|^{2}+|b|^{2}=1$.

El álgebra de Lie de $S U(2)$ es el espacio lineal de tres dimensiones

$$
s u(2)=\left\{X \in M(2, \mathbb{C}) \mid X^{T}=-X, \operatorname{con} \operatorname{traza}(X)=0\right\} .
$$

En la primera sección de este trabajo estudiaremos algunas propiedades de una clase de equilibrios relativos, que son los siguientes

Definición 3.1.2 Un equilibrio relativo elíptico en $S^{2}$ es una solución de la forma $q_{i}=$ $\left(x_{i}, y_{i}, x_{i}\right), i=1, \ldots, n$ de las ecuaciones de movimiento con $x_{i}=r_{i} \cos \left(\omega t+\alpha_{i}\right), y y_{i}=$ $r_{i} \cos \left(\omega t+\alpha_{i}\right), z_{i}=$ constante, donde $\omega, \alpha_{i} y r_{i}, i=1, \ldots, n$ son constantes, con $0 \leq r_{i}=$ $\left(1-z_{i}^{2}\right)^{1 / 2} \leq 1$.

Definiremos tambíen el siguiente tipo de equilibrios relativos que son en los que nos enfocaremos en este trabajo.

Definición 3.1.3 Si los cuerpos giran sobre una curva geodésica, las correspondientes soluciones son llamadas equilibrios relativos eulerianos.

A continuación definiremos una configuración de punto fijo

Definición 3.1.4 Una solución de las ecuaciones de movimiento es llamada punto fijo si se cumple

$$
\nabla_{q_{i}} U(q)(t)=0, \quad t \in \mathbb{R}, \quad i=1, \ldots, n .
$$

Empezaremos mostrando el siguiente resultado que es el más simple acerca de puntos fijos en $S^{2}$.

Teorema 3.1.1 Consideremos el problema de $n$ cuerpos en $S^{2}$, con $n$ impar. Si todas las masas son iguales, entonces el polígono regular que forman los cuerpos y que se encuentra en cualquier geodésica es un punto fijo de las ecuaciones de movimiento. 


\section{Demostración}

Por hipótesis tenemos que $m_{1}=m_{2}=\cdots=m_{n}$; consideremos el $n$-ágono regular con un número impar de lados inscrito en una geodésica de $S^{2}$, con cada cuerpo inicialmente en reposo en cada vértice. En general, dos fuerzas actuan en el cuerpo de masa $m_{i}$ : la fuerza $\nabla_{q_{i}} U(q)$ que es la que se genera con la interacción con los otros cuerpos, y la fuerza $-m_{i}\left(\dot{q}_{i} \cdot \dot{q}_{i}\right) q_{i}$ que es la correspondiente a la restriccion que mantiene a las partículas sobre la superficie correspondiente. Esta última fuerza es cero al tiempo $t=0$, ya que los cuerpos se encuentran inicialmente en reposo. Como $q_{i} \cdot \nabla_{q_{i}} U(q)=0$, tenemos que $\nabla_{q_{i}} U(q)$ es ortogonal a $q_{i}, \mathrm{y}$ por lo tanto tangente a $S^{2}$. Entonces la simetría del polígono regular implica que al tiempo $t=0, \nabla_{q_{i}} U(q)$ es la suma de pares de fuerzas, cada par consiste de fuerzas opuestas que se cancelan unas con otras. Esto significa que $\nabla_{q_{i}} U(q)=0$. Así, de las ecuaciones de moviento y del hecho que los cuerpos están inicialmente en reposo tenemos que

$$
\ddot{q}_{i}(0)=-\left(\dot{q}_{i}(0) \cdot \dot{q}_{i}(0)\right) q_{i}(0)=0, \quad i=1, \ldots, n .
$$

Pero, entonces, no hay fuerzas que actuen sobre el cuerpo de masa $m_{i}$ al tiempo $t=0$, y por lo tanto el cuerpo no se mueve. Luego $\dot{q}_{i}(t)=0$ para todo $t \in \mathbb{R}$. Entonces $\ddot{q}_{i}(t)=0$ para cada $t \in \mathbb{R}$, y así $\nabla_{q_{i}} U(t)=0$ para todo $t \in \mathbb{R}$, y el $n$-ágono es un punto fijo de las ecuaciones de movimiento.

Notemos que si $n$ es par, el $n$-ágono tiene $n / 2$ pares de vértices antipodales. Como los cuerpos en posiciones antipodales generan singularidades en las ecuaciones de movimiento, los únicos polígonos regulares con un número impar de vértices son puntos fijos de las ecuaciones de movimiento.

Si las partículas inicialmente se encuentran sobre un polígono regular y se mantienen en la misma configuración en todo tiempo entonces es equivalente a decir que las fuerzas entre ellas se compensan y se eliminan, enunciaremos esto a traves del siguiente corolario, cuya demostración es inmediata del teorema anterior.

Corolario 3.1.1 Consideremos un número impar de partículas con masas iguales inicialmente sobre los vértices de un polígono regular inscrito sobre un círculo máximo de $S^{2}$, y asumamos que la solución generada por esta condición inicial mantiene a las partículas en la misma configuración en todo tiempo t. Entonces, para todo $t \in \mathbb{R}$, esta solución satisface las condiciones

$$
\nabla_{q_{i}} U(q(t))=0, i=1,2, \cdots, n .
$$

A continuación mostraremos un resultado importante que exhibe configuraciones de los cuerpos sobre $S^{2}$ en donde éstas no son puntos fijos de las ecuaciones de movimiento. 
Teorema 3.1.2 Consideremos una configuración no singular del problema de los n-cuerpos en $S^{2}$ en donde los cuerpos se encuentran en uno de los hemisferios, incluyendo al ecuador, y en donde por lo menos un cuerpo se encuentra fuera de dicho círculo máximo. Entonces esta configuración no es un punto fijo.

\section{Demostración}

Sin pérdida de generalidad consideremos la configuración inicial de los cuerpos $m_{1}, m_{2}, \cdots, m_{n}$ en el hemisferio norte. Tenemos que uno de los cuerpos tiene la coordenada $z$ menor, sea $m_{1}$ dicho cuerpo. De la misma manera por lo menos uno de los cuerpos tiene coordenada $z$ positiva, sea $m_{2}$ una de esas partículas. Como todas las velocidades iniciales son cero, solo las fuerzas mutuas actuan sobre $m_{1}$. Entonces, de acuerdo con las ecuaciones de movimiento, $m_{1} \ddot{z}_{1}(0)=\frac{\partial}{\partial z_{1}} U(q(0))$. Pero como ningun cuerpo tiene coordenada $z$ menor que $z_{1}$ los términos que se encuentran en $\frac{\partial}{\partial z_{1}} U(q(0))$ que involucran interacciones entre $m_{1}$ y $m_{i}$ son mayores o iguales a cero para $i=3,4, \cdots, n$, mientras que los términos que involucran a $m_{2}$ son estrictamente positivas. Así $\frac{\partial}{\partial z_{1}} U(q(0))>0$, entonces $m_{1}$ se mueve hacia el polo norte de la esfera. Por lo tanto la configuración inicial no es un punto fijo.

El resultado que enseguida mostraremos nos da condiciones para que un punto fijo genere equilibrios relativos.

Teorema 3.1.3 Consideremos un punto fijo dado por las masas $m_{1}, m_{2}, \cdots, m_{n}$ que se encuentran sobre un círculo máximo de $S^{2}$. Entonces si la velocidad angular es cero, esta configuración genera un equilibrio relativo a lo largo del círculo máximo.

\section{Demostración}

Sin pérdida de generalidad, asumamos que las partículas se encuentran sobre el ecuador, y que para ciertas masas dadas $m_{1}, m_{2}, \cdots, m_{n}$, existen $\alpha_{1}, \alpha_{2}, \cdots, \alpha_{n}$ tales que la configuración $q=\left(q_{1}, q_{2}, \cdots, q_{n}\right)$ dados por $q_{i}=\left(x_{i}, y_{i}, 0\right), i=1,2, \cdots, n$, con

$$
x_{i}=\cos \left(\omega t+\alpha_{i}\right), \quad y_{i}=\sin \left(\omega t+\alpha_{i}\right), \quad i=1,2, \cdots, n,
$$

es un punto fijo para $\omega=0$. Esta configuración también puede ser interpretada como $q(0)$, es decir, la solución $q$ al tiempo $t=0$, para cualquier $\omega \neq 0$. Entonces podemos concluir que $\left.\nabla_{q_{i}} U_{(}(0)\right)=0, i=1,2, \cdots, n$. Pero, entonces para $t=0$, las ecuaciones de movimiento se reducen a

$$
\begin{aligned}
\ddot{x}_{i} & =-\left(\dot{x}_{i}^{2}+\dot{y}_{i}^{2}\right) x_{i} \\
\ddot{y}_{i} & =-\left(\dot{x}_{i}^{2}+\dot{y}_{i}^{2}\right) y_{i}
\end{aligned}
$$

$i=1,2, \cdots, n$. Notemos que $\dot{x}_{i}=-\omega \sin \left(\omega t+\alpha_{i}\right), \ddot{x}_{i}=-\omega^{2} \sin \left(\omega t+\alpha_{i}\right), \dot{y}_{i}=-\omega \cos \left(\omega t+\alpha_{i}\right)$, $\ddot{y}_{i}=-\omega^{2} \sin \left(\omega t+\alpha_{i}\right)$, así $\dot{x}_{i}^{2}+\dot{y}_{i}^{2}=\omega^{2}$. Usando estos cálculos es fácil ver que $q$ dado por (3.18) 
es solución de (3.20), para todo $t$, entonces no hay fuerzas que se deriven de las restricciones que actuan sobre los cuerpos, ni al tiempo $t=0$ ni a un tiempo $t>0$. Como $\nabla_{q_{i}} U(q(0))=0$ para $i=1,2, \cdots, n$, se sigue que las fuerzas gravitaciones están en equilibrio al momento inicial, y ninguna fuerza gravitacional actua sobre los cuerpos. Consecuentemente, la rotación dada por $\omega \neq 0$ hace que el sistema se mueva como cuerpo rígido, y que las fuerzas de rotación se mantengan en equilibrio, como consecuencia tenemos $\nabla_{q_{i}} U(q(t))=0, i=1,2, \cdots, n$ para todo $t$.

Entonces $q$ dado por (3.18) satisface las ecuaciones de movimiento, y por la definición de equilibrio relativo elíptico, $q$ es uno de ellos.

A continuación mostraremos que un equlibrio relativo generado por puntos fijos que se obtienen a partir de polígonos regulares sobre un círculo máximo de $S^{2}$ puede ocurrir sólo si las partículas rotan a lo largo del círculo máximo.

Teorema 3.1.4 Consideremos un número impar de partículas de masas iguales inicialmente en los vértices de un polígono regular inscrito en un círculo máximo de $S^{2}$. Entonces el único equilibrio relativo elíptico que se puede generar a partir de esta configuración es aquel dado por la rotación de las partículas en el plano que contiene al círculo máximo en donde inicialmente se encuentran.

\section{Demostración}

Sin pérdidad de generalidad, podemos tomar al círculo máximo que está sobre $z=0$. Consideremos una solución de equilibrio relativo elíptico de la forma

$$
x_{i}=r_{i} \cos \left(\omega t+\alpha_{i}\right), \quad y_{i}=r_{i} \sin \left(\omega t+\alpha_{i}\right), z_{i}= \pm\left(1-r_{i}^{2}\right)^{1 / 2}, i=1,2, \cdots, n,
$$

en donde tomamos + para $z_{i}>0 \mathrm{y}-$ para $z_{i}<0$. La única condición que imponemos es que $r_{i}$ y $\alpha_{i}$ son escogidas de tal forma que la configuración es un polígono regular de $n$ vértices inscrito en un círculo máximo de $S^{2}$, y que se mueven sobre este círculo en todo tiempo $t$. Podemos medir el ángulo que forma el plano que contiene a las partículas con el eje $z$. La solución que pusimos anteriormente tiene derivadas

$$
\begin{gathered}
\dot{x}_{i}=-r_{i} \omega \sin \left(\omega t+\alpha_{i}\right), \quad \dot{y}_{i}=r_{i} \omega \cos \left(\omega t+\alpha_{i}\right), \dot{z}_{i}=0, i=1,2, \cdots, n, \\
\ddot{x}_{i}=-r_{i} \omega^{2} \cos \left(\omega t+\alpha_{i}\right), \quad \ddot{y}_{i}=-r_{i} \omega^{2} \sin \left(\omega t+\alpha_{i}\right), \ddot{z}_{i}=0, i=1,2, \cdots, n .
\end{gathered}
$$

Luego tenemos

$$
\dot{x}_{i}^{2}+\dot{y}_{i}^{2}+\dot{z}_{i}^{2}=r_{i}^{2} \omega^{2}, \quad i=1,2, \cdots, n .
$$


Por el primer corolario de este capítulo tenemos que el polígono regular satisface

$$
\nabla_{q_{i}} U(q)=0, \quad i=1,2, \cdots, n .
$$

El sistema de ecuaciones de movimiento se reduce a

$$
\begin{array}{r}
\ddot{x}_{i}=-\left(\dot{x}_{i}^{2}+\dot{y}_{i}^{2}+\dot{z}_{i}^{2}\right) x_{i}, \\
\ddot{y}_{i}=-\left(\dot{x}_{i}^{2}+\dot{y}_{i}^{2}+\dot{z}_{i}^{2}\right) y_{i}, \\
\ddot{z}_{i}=-\left(\dot{x}_{i}^{2}+\dot{y}_{i}^{2}+\dot{z}_{i}^{2}\right) z_{i}, \quad i=1,2, \cdots, n .
\end{array}
$$

Si sustituimos la ecuacion (3.20) en las últimas ecuaciones tenemos

$$
\begin{array}{r}
r_{i}\left(1-r_{i}^{2}\right) \omega^{2} \cos \left(\omega t+\alpha_{i}\right)=0, \\
r_{i}\left(1-r_{i}^{2}\right) \omega^{2} \sin \left(\omega t+\alpha_{i}\right)=0, \quad i=1,2, \cdots, n .
\end{array}
$$

Pero asumiendo que $\omega \neq 0$, este sistema se satisface si $r_{i}=1$, condición que es equivalente a $z_{i}=0$, por lo tanto los cuerpos tienen que rotar a lo largo del ecuador $z=0$.

En el siguiente teorema mostraremos las condiciones para que cuerpos de masas iguales que forman un polígono regular formen equilibrios relativos elípticos girando en curvas que no son geodésicas.

Teorema 3.1.5 Consideremos el problema de los $n$-cuerpos en $S^{2}$ con masas iguales. Entonces para cada $n$ impar, $m>0$ y $z \in(-1,1)$, existe un valor positivo y uno negativo de $\omega$ que generan equilibrios relativos elípticos en donde los cuerpos se encuentran en los vértices de un polígono regular en el plano $z=$ cte. Si $n$ es par, este resultado es cierto si excluimos $a z=0$.

\section{Demostración}

Discutiremos dos casos, $n$ par y $n$ impar.

Para simplificar notación denotaremos a los cuerpos por $m_{i}$ con índices $i=-s,-s+$ $1, \cdots,-1,0,1, \cdots, s-1, s$, donde $s \in \mathbb{N}$. Sin pérdida de generalidad suitituimos en las ecuaciones de movimiento (2.26) soluciones de la forma (3.20) con los índices $i$ como los acabamos de exhibir, $\alpha_{-s}=-\frac{4 s \pi}{2 s+1}, \cdots, \alpha_{-1}=-\frac{2 \pi}{2 s+1}, \alpha_{0}=0, \alpha_{1}=\frac{2 \pi}{2 s+1}, \cdot \alpha_{s}=\frac{4 s \pi}{2 s+1} \cdot r=r_{i}, z=z_{i}$. Debido a las simetrías del problema es suficiente ver que pasa considerando las ecuaciones para $i=0$.

La ecuación correspondiente a la coordenada $z_{0}$ toma la forma 


$$
\sum_{j=-s, j \neq 0}^{s} \frac{m\left(z-k_{0 j} z\right)}{\left(1-k_{0 j}^{2}\right)^{3 / 2}}-r^{2} \omega^{2} z=0
$$

donde $k_{0 j}=x_{0} x_{j}+y_{0} y_{j}+z_{0} z_{j}=\cos \alpha_{j}-z^{2} \cos \alpha_{j}+z^{2}$. Usando el hecho de que $r^{2}+z^{2}=$ $1, \cos \alpha_{j}=\cos \alpha_{-j}$, y $k_{0 j}=k_{0-(j)}$, tenemos que para la coordenada $z_{0}$

$$
\sum_{j=1}^{s}=\frac{2\left(1-\cos \alpha_{j}\right)}{\left(1-k_{0 j}^{2}\right)^{3 / 2}}=\frac{\omega^{2}}{m}
$$

Tomando en cuenta $x_{0}$ e ignorando $y_{0}$ es suficiente debido a la simetría del problema y a la dualidad de las funciones trigonométricas sin y cos. Si sustituimos éstas en las ecuaciones de movimiento tenemos

$$
\left(r^{2}-1\right) \omega^{2} \cos \omega t=\sum_{j=-s, j \neq 0}^{s} \frac{m\left[\cos \left(\omega t+\alpha_{j}\right)-k_{0 j}^{2} \cos \omega t\right]}{\left(1-k_{0 j}^{2}\right)^{3 / 2}} .
$$

Después de varios cálculos y usando el hecho de que $r^{2}+z^{2}=1, \sin \alpha_{j}=-\sin \alpha_{j}$, $\cos \alpha_{j}=$ $\cos \alpha_{-j}$, y $k_{0 j}=k_{0(-j)}$, tenemos la misma ecuación (3.27). Escribiendo el denominador de (3.27) explicitamente tenemmos

$$
\sum_{j=1}^{s} \frac{2}{\left(1-\cos \alpha_{j}\right)^{1 / 2}\left(1-z^{2}\right)^{3 / 2}\left[2-\left(1-\cos \alpha_{j}\right)\left(1-z^{2}\right)\right]^{3 / 2}}=\frac{\omega^{2}}{m}
$$

La parte izquierda de la ecuación es positiva para cualquier $m>0$ y $z \in(-1,1)$ fijos, entonces tenemos el valor de los correspondientes valores positivos y negativos de $\omega$ que son los que satisfacen la ecuación.

Ahora veamos el caso en donde $n$ es par. Denotemos de manera análoga a los cuerpos por $m_{i}$, con $i=-s+1, \cdots,-1,0,1, \cdots, s-1$, donde $s \in \mathbb{N}$ y todos los cuerpos tienen la misma masa $m$. Sin pérdida de generalidad, podemos sustituir en las ecuaciones de movimiento soluciones de la forma (3.20), con $i$ como escribimos anteriormente, y $\alpha_{-s+1}=-\frac{-(s+1) \pi}{s}, \cdots, \alpha_{-1}=-\frac{-\pi}{s}, \alpha_{0}=0, \alpha_{1}=\frac{\pi}{s}, \cdot \alpha_{s-1}=\frac{(s-1) \pi}{s}, \alpha_{s}=\pi, r=r_{i}, z=z_{i}$, y de nuevo consideraremos el caso para $i=0$. Usando el hecho de que $k_{0 j}=k_{0(-j)}, \cos \alpha_{j}=\cos \alpha_{-j}$ $\mathrm{y} \cos \pi=-1$, tenemos

$$
\sum_{j=1}^{s-1} \frac{2\left(1-\cos \alpha_{j}\right)}{1-\left(k_{0 j}^{2}\right)^{3 / 2}}+\frac{2}{\left(1-k_{0 s}^{2}\right)^{3 / 2}}=\frac{\omega^{2}}{m} .
$$

Usando las relaciones $\sin \alpha_{j}=-\sin \alpha_{-j} \mathrm{y} \sin \pi=0$ obtenemos para la ecuación correspodiente a $x_{0}$ lo mismo que (3.30), que escribiendo explicitamente tenemos 


$$
\begin{aligned}
\sum_{j=1}^{s-1} \frac{2}{\left(1-\cos \alpha_{j}\right)^{1 / 2}\left(1-z^{2}\right)^{3 / 2}\left[2-\left(1-\cos \alpha_{j}\left(1-z^{2}\right)\right)\right]^{3 / 2}} \\
\quad+\frac{1}{4 z^{2}|z|\left(1-z^{2}\right)^{3 / 2}}=\frac{\omega^{2}}{m} .
\end{aligned}
$$

Como la parte de la izquieda es positivo y finito para cualquier $m>0$, y $z \in(-1,1) /\{0\}$ entonces tenemos que existe un valor positivo y uno negativo para el que tenemos este tipo de equilibrios relativos, exceptuando el valor de $z=0$.

Es importante señalar que en el problema de tres cuerpos existen otro tipo de equilibrios relativos, son los llamados equilibrios relativos lagrangianos, y estos surgen de las siguientes soluciones.

Definición 3.1.5 Una solución de las ecuaciones 2.26 del problema de los tres cuerpos se le llama Lagrangiana si las tres masas forman un triángulo equilátero en todo tiempo $t$.

Este tipo de movimientos se estudian a fondo en [25].

\subsection{Equilibrios Relativos Eulerianos}

En esta sección mostraremos resultados acerca de equilibrios relativos elípticos eulerianos en el caso $n=3$.

Teorema 3.2.1 Consideremos el problema de 3-cuerpos en $S^{2}$ con masas $M=m_{1}$ y $m=$ $m_{2}=m_{3}$. Fijemos el cuerpo de masa $m_{1}$ en el punto $(0,0,1)$ y los cuerpos de masas $m_{2}$ y $m_{3}$ en los lados opuestos de un círculo a la altura $z=$ cte. Entonces si $4 m>M y z \in$ $(-\sqrt{M} / 2 \sqrt{m}, 0) \cup(0,1)$, existe un valor positivo y uno negativo de $\omega$ que produce equilibrios relativos elípticos.

\section{Demostración}

Tomemos en cuenta las coordenadas $x$ y $z$ de la ecuación de movimiento correspondiente a la segunda partícula, dadas por las siguientes ecuaciones

$$
\ddot{x}_{2}=\frac{m_{1}\left(x_{1}-\left(x_{1} x_{2}+y_{1} y_{2}+z_{1} z_{2}\right) x_{2}\right)}{\left(1-\left(x_{1} x_{2}+y_{1} y_{2}+z_{1} z_{2}\right)^{2}\right)^{\frac{3}{2}}}+\frac{m_{3}\left(x_{3}-\left(x_{1} x_{2}+y_{1} y_{2}+z_{1} z_{2}\right) x_{2}\right)}{\left(1-\left(x_{1} x_{2}+y_{1} y_{2}+z_{1} z_{2}\right)^{2}\right)^{\frac{3}{2}}}-\left(\dot{x}_{2}^{2}+\dot{y}_{2}^{2}+\dot{z}_{2}^{2}\right),
$$


y

$$
\ddot{z}_{2}=\frac{m_{1}\left(z_{1}-\left(x_{1} x_{2}+y_{1} y_{2}+z_{1} z_{2}\right) z_{2}\right)}{\left(1-\left(x_{1} x_{2}+y_{1} y_{2}+z_{1} z_{2}\right)^{2}\right)^{\frac{3}{2}}}+\frac{m_{3}\left(z_{3}-\left(x_{1} x_{2}+y_{1} y_{2}+z_{1} z_{2}\right) z_{2}\right)}{\left(1-\left(x_{1} x_{2}+y_{1} y_{2}+z_{1} z_{2}\right)^{2}\right)^{\frac{3}{2}}}-\left(\dot{x}_{2}^{2}+\dot{y}_{2}^{2}+\dot{z}_{2}^{2}\right) .
$$

En nuestro problema estamos interesados en soluciones en donde los cuerpos $q_{i}, 1=1,2,3$ tienen coordenadas

$$
\begin{gathered}
x_{1}=0, \quad y_{1}=0, z_{1}=1 \\
x_{2}=r \cos \omega t, \quad y_{2}=\sin \omega t, \quad z_{2}=z, \\
x_{3}=r \cos (\omega t+\pi), \quad y_{3}=\sin (\omega t+\pi), \quad z_{3}=z,
\end{gathered}
$$

en donde $\omega$ es la velocidad angular.

Sustituyendo en $\ddot{x}_{2}$

$$
r \omega^{2} \cos \omega t=\frac{m_{1} r z \cos \omega t}{\left(1-z^{2}\right)^{\frac{3}{2}}}+\frac{m_{3}\left(r \cos \omega t-r \cos \omega t\left(r^{2}-z^{2}\right)\right)}{\left(1-\left(r^{2}-z^{2}\right)^{2}\right)^{\frac{3}{2}}}+r^{3} \omega^{2} \cos \omega t .
$$

Despejando a $\omega^{2}$ obtenemos

$$
\omega^{2}=\frac{m_{1} z}{\left(1-z^{2}\right)^{\frac{3}{2}}}+\frac{m_{3}}{\left(1-\left(r^{2}-z^{2}\right)^{2}\right)^{\frac{3}{2}}}-\frac{m_{3}\left(r^{2}-z^{2}\right)}{\left(1-\left(r^{2}-z^{2}\right)^{2}\right)^{\frac{3}{2}}}+r^{2} \omega^{2} .
$$

Sustituyendo las correspondientes coordenadas de (3.35) en $\ddot{z}_{2}$ obtenemos

$$
\begin{aligned}
0 & =\frac{m_{1}}{\sqrt{1-z^{2}}}-r^{2} \omega^{2} z+\frac{m_{3}\left(z+z\left(r^{2}-z^{2}\right)\right)}{\left(1-\left(r^{2}-z^{2}\right)^{2}\right)^{\frac{3}{2}}} \\
& =\frac{\frac{1}{z} m_{1}}{\sqrt{1-z^{2}}}-r^{2} \omega^{2}+\frac{m_{3}}{\left(1-\left(r^{2}-z^{2}\right)^{2}\right)^{\frac{3}{2}}}+\frac{m_{3}\left(r^{2}-z^{2}\right)}{\left(1-\left(r^{2}-z^{2}\right)^{2}\right)^{\frac{3}{2}}} .
\end{aligned}
$$

Sumando (3.36) y (3.37) obtenemos

$$
\omega^{2}=\frac{\frac{1}{z} m_{1}}{\sqrt{1-z^{2}}}+\frac{2 m_{3}}{\left(1-\left(r^{2}-z^{2}\right)^{2}\right)^{\frac{3}{2}}}+\frac{m_{1} z}{\left(1-z^{2}\right)^{\frac{3}{2}}} .
$$

Factorizando el término $\frac{m_{1}}{\left(1-z^{2}\right)^{\frac{1}{2}}}$ llegamos a la siguiente ecuación

$$
\begin{aligned}
\omega^{2} & =\frac{2 m_{3}}{\left(1-\left(r^{2}-z^{2}\right)^{2}\right)^{\frac{3}{2}}}+\frac{m_{1}}{\left(1-z^{2}\right)^{\frac{1}{2}}}\left(\frac{1}{z}+\frac{z}{1-z^{2}}\right) \\
& =\frac{2 m_{3}}{\left(1-\left(r^{2}-z^{2}\right)^{2}\right)^{\frac{3}{2}}}+\frac{m_{1}}{\left(1-z^{2}\right)^{\frac{1}{2}}}\left(\frac{1}{z\left(1-z^{2}\right)}\right) .
\end{aligned}
$$


Para escribir todo en términos de $z$ usamos la expresión $1=r^{2}+z^{2}$.

$$
\begin{aligned}
\omega^{2} & =\frac{2 m_{3}}{\left(1-\left(1-2 z^{2}\right)^{2}\right)^{\frac{3}{2}}}+\frac{m_{1}}{\left(1-z^{2}\right)^{\frac{1}{2}}}\left(\frac{1}{z\left(1-z^{2}\right)}\right) . \\
& =\frac{m_{3}}{4|z|^{3}\left(1-z^{2}\right)^{\frac{3}{2}}}+\frac{m_{1}}{z\left(1-z^{2}\right)^{\frac{3}{2}}} \\
& =\frac{m_{3}|z|^{-1}+4 m_{1} z}{4 z^{2}\left(1-z^{2}\right)^{\frac{3}{2}}} .
\end{aligned}
$$

El numerador nos muestra que esta ecuación tiene soluciones para $z \in(-\sqrt{M} / 2 \sqrt{m}, 0) \cup$ $(0,1)$, podemos ver la gráfica de esta funcion para ciertos valores de $m$ y $M$ en las figuras (3.2) y (3.3); además se debe cumplir la relación de masas $4 m>M$. El signo de $\omega$ determina el sentido de rotación.

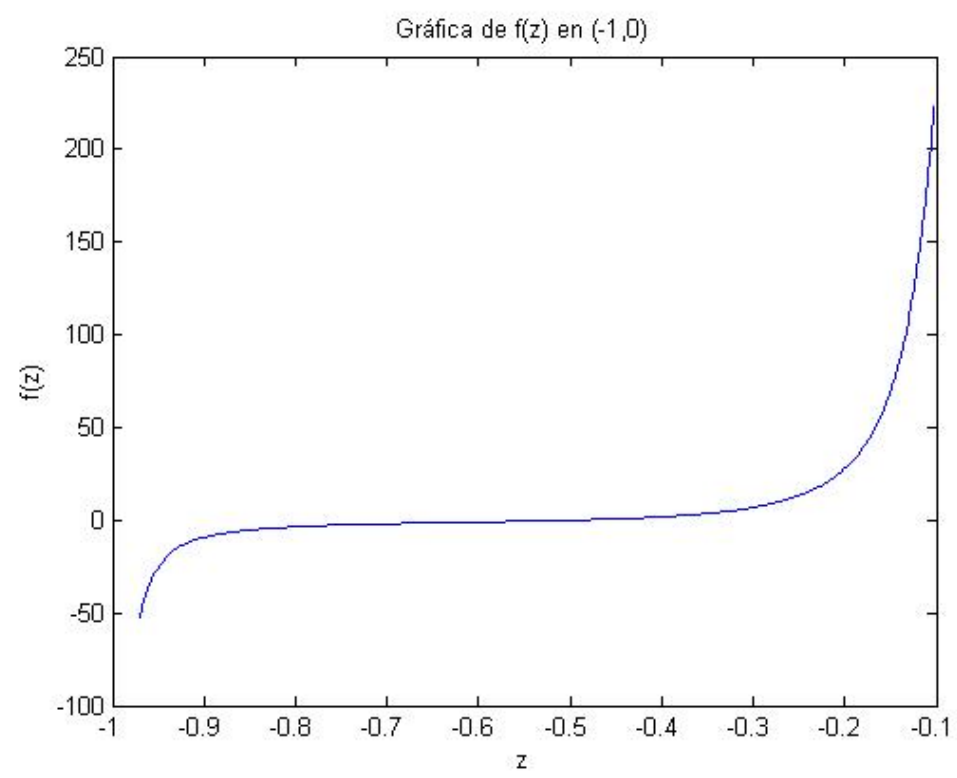

Figura 3.2: Gráfica $f(z)=\frac{m_{3}|z|^{-1}+4 m_{1} z}{4 z^{2}\left(1-z^{2}\right)^{\frac{3}{2}}}$ con $m_{3}=m_{1}=1$ en $(-1,0)$. 


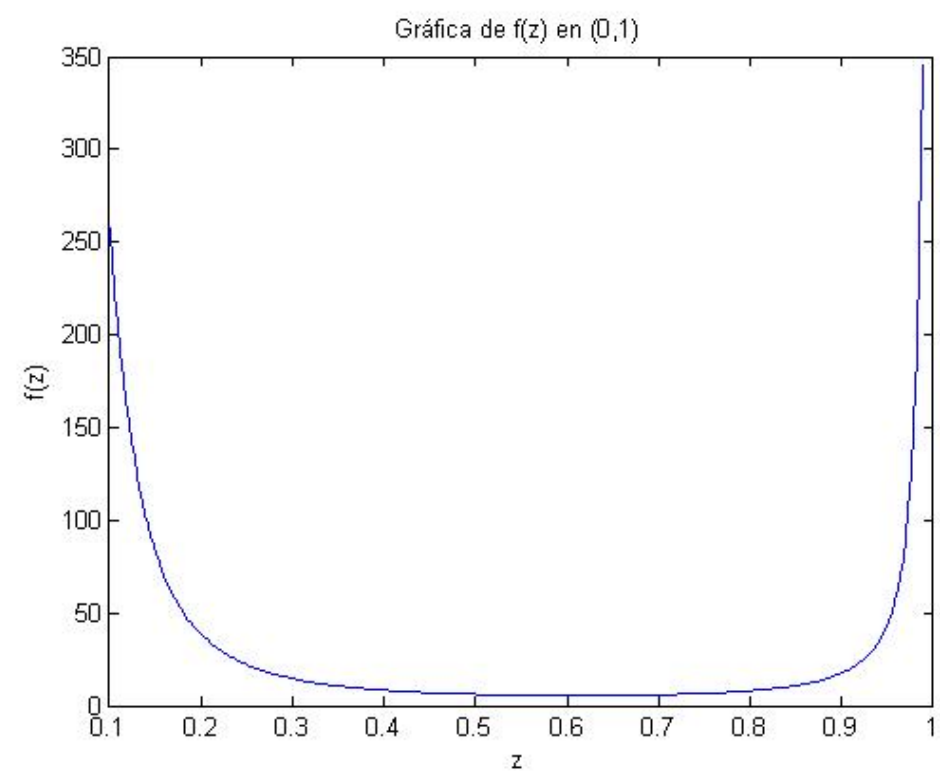

Figura 3.3: Gráfica $f(z)=\frac{m_{3}|z|^{-1}+4 m_{1} z}{4 z^{2}\left(1-z^{2}\right)^{\frac{3}{2}}}$ con $m_{3}=m_{1}=1$ en $(0,1)$. 


\section{Capítulo 4 Problema de $n$-Cuerpos en $H^{2}$}

Al igual que en $S^{2}$ mostraremos resultados referentes a equilibrios relativos del tipo euleriano del problema de dos y tres cuerpos en $H^{2}$, considerando que existe otro tipo de equilibrio relativo, llamado lagrangiano, del problema de tres cuerpos, esto, al igual que en $S^{2}$ surgen de los siguientes movimientos

Definición 4.0.1 Una solución de las ecuaciones 2.26 del problema de los tres cuerpos se le llama Lagrangiana si las tres masas forman un triángulo equilátero en todo tiempo $t$.

\subsection{Equilibrios Relativos en $H^{2}$}

En esta sección mostraremos resultados relacionados con puntos fijos, además de definir y probar algunos resultados acerca de los llamados equilibrios relativos elípticos e hiperbólicos, además demostraremos que no existen equilibrios relativos parabólicos, recordemos que en este trabajo nos enfocamos en los equlibiros relativos de tipo eulerianos, por esta razón mostraremos resultados referentes a ellos. Resultados básicos de geometría diferencial nos dicen que cada rotación de Lorentz se puede escribir, en alguna base, como una rotación elíptica alrededor del eje $z$, como una rotación hiperbólica alrededor del eje $x$, o como una rotación parabólica alrededor de la linea $x=0, y=z$. De esta manera es como se definen los equilibrios relativos elípticos, hiperbólicos y parabólicos. A continuación mostramos las definiciones en terminología más precisa.

Definición 4.1.1 Un equilibrio relativo elíptico en $H^{2}$ es una solución $q_{i}=\left(x_{i}, y_{i}, z_{i}\right), i=$ $1,2, \cdots, n$, de las ecuaciones de movimiento con $x_{i}=\rho_{i} \cos \left(\omega t+\alpha_{i}\right), y_{i}=\rho_{i} \sin \left(\omega t+\alpha_{i}\right) y$ $z_{i}=\left(\rho_{i}^{2}+1\right)^{1 / 2}$, donde $\omega, \alpha_{i} y \rho_{i}, i=1,2, \cdots, n$, son constantes.

Definición 4.1.2 Un equilibrio relativo hiperbólico en $H^{2}$ es una solución $q_{i}=\left(x_{i}, y_{i}, z_{i}\right), i=$ $1,2, \cdots, n$, de las ecuaciones de movimiento con

$$
x_{i}=c t e, y_{i}=\rho_{i} \sinh \left(\omega t+\alpha_{i}\right), z_{i}=\rho_{i} \cosh \left(\omega t+\alpha_{i}\right),
$$

donde $\omega, \alpha_{i}$ y $\rho_{i}=\left(1+x_{i}^{2}\right)^{1 / 2} \geq 1, i=1,2, \cdots, n$, son constantes. 
Definición 4.1.3 Un equilibrio relativo parabólico en $H^{2}$ es una solución $q_{i}=\left(x_{i}, y_{i}, z_{i}\right), i=$ $1,2, \cdots, n$, de las ecuaciones de movimiento con

$$
x_{i}=a_{i}-b_{i} t+c_{i} t, \quad y_{i}=a_{i} t+b_{i}\left(1-t^{2} / 2\right)+c_{i} t^{2} / 2, \quad z_{i}=a_{i} t-b_{i} t^{2} / 2+c_{i}\left(1+t^{2} / 2\right)
$$

donde $a_{i}, b_{i}$ y $c_{i}, i=1,2, \cdots, n$, son constantes, además se tiene $a_{i}^{2}+b_{i}^{2}-c_{i}^{2}=-1$.

Al igual que en $S^{2}$ nos enfocaremos en los equilibrios relativos eulerianos, definidos de la misma manera.

Definición 4.1.4 Si los cuerpos giran sobre una curva geodésica, las correspondientes soluciones son llamadas equilibrios relativos eulerianos.

Las soluciones más simples de las ecuaciones de movimiento en $H^{2}$ son los puntos fijos, éstos los definimos a continuación

Definición 4.1.5 Una solución de las ecuaciones de movimiento es llamada punto fijo si

$$
\nabla_{q_{i}} U_{-1}(q)(t)=p_{i}(t)=0
$$

para $t \in \mathbb{R}, i=1,2, \cdots, n$.

El primer resultado importante en $H^{2}$ es el siguiente.

Teorema 4.1.1 En el problema de $n$-cuerpos en $H^{2}$ con $n \geq 2$ no hay configuraciones que correspondan a puntos fijos de las ecuaciones de movimiento.

\section{Demostración}

Consideremos una configuración de $n$ cuerpos en la que evitamos las colisiones, y que inicialmente se encuentra en reposo. Esto significa que las componentes de las fuerzas actuando en sobre los cuerpos, que incluyen a los factores $\dot{x}_{i}^{2}+\dot{y}_{i}^{2}-\dot{z}_{i}^{2}, 1=1,2, \cdots, n$, son cero al tiempo $t=0$. Pero tenemos que por lo menos un cuerpo tiene a la coordenada $z$ de mayor valor. Notemos que la interacción entre $m_{i}$ y cualquier otro cuerpo es a lo largo de geodésicas, las cuales son hipérbolas que abren hacia arriba en la parte con $z>0$ del hiperboloide que modela a $H^{2}$. Entonces el cuerpo $m_{j}, j \neq i$, ejerce una atracción sobre $m_{i}$ en dirección hacia abajo sobre la hipérbola que conecta a los dos cuerpos, entonces la coordenada $z$ de la fuerza que actua sobre $m_{i}$ es negativa, independiente de si $z-j(0)<z_{i}(0)$ o $z-j(0)=z_{i}(0)$. Esto es cierto para $j=1,2, \cdots, n, j \neq i$, se sigue que $\ddot{z}_{i}<0$. Así $m_{i}$ se mueve en dirección hacia abajo sobre el hiperboloide, entonces la configuración original no es un punto fijo. 


\subsubsection{Equilibrios Relativos Elípticos}

Consideremos equilibrios relativos elípticos, mostraremos algunas condiciones sobre las velocidades y masas de los cuerpos para obtener este tipo de movimientos

Teorema 4.1.2 Consideremos el problema de $n$ cuerpos en $H^{2}$. Entonces para cualquier $m>0$ y $z>1$ hay un valor positivo y uno negativo de $\omega$ que producen equilibrios relativos elípticos en donde los cuerpos están sobre los vértices de un n-ágono que rota en el plano $z=$ cte.

\section{Demostración}

La prueba es similar que en el caso de $S^{2}$, considerando los casos pares e impares de forma separada. Las únicas diferencias es que reemplazamos la variable $r$ por la variable $\rho$, además de las respectivas expresiones $r^{2}+z^{2}=1$ y $z^{2}=\rho^{2}+1$; también reemplazamos el denominador $\left(1-k_{0 j}^{2}\right)^{3 / 2}$ por $\left(c_{0 j}^{2}-1\right)^{3 / 2}$, donde $c_{0 j}=-k_{0 j}$ reemplaza a $k_{0 j}$.

Corolario 4.1.1 Consideremos el problema de 3 cuerpos con masas iguales en $H^{2}$. Entonces para cada $m>0$ y $z>1$, existe un valor positivo y uno negativo de $\omega$ que produce equilibrios relativos en donde los cuerpos se encuentran en los vértices de un triángulo equilatero que rota en el plano $z=$ cte. Más aún, para cada $\omega^{2} / m>0$ existe un único valor de $z$.

\section{Demostración}

Si sustituimos en las correspondientes ecuaciones de movimiento para el problema de tres cuerpos en $H^{2}$ una solucíon de la forma

$$
x_{i}=\rho \cos \left(\omega t+\alpha_{i}\right), \quad y_{i}=\rho \sin \left(\omega t+\alpha_{i}\right), \quad z_{i}=z,
$$

con $z=\sqrt{\rho^{2}+1}, \alpha_{1}=0, \alpha_{2}=2 \pi / 3, \alpha_{3}=4 \pi / 3$, llegamos a la siguiente ecuación

$$
\frac{8}{\sqrt{3}\left(3 z^{4}-2 z^{2}-1\right)^{3 / 2}}=\frac{\omega^{2}}{m}
$$

Entonces para cada $z \in(1, \infty)$ existe un valor positivo y uno negativo de $\omega$ para el cual se satisface (4.5). 


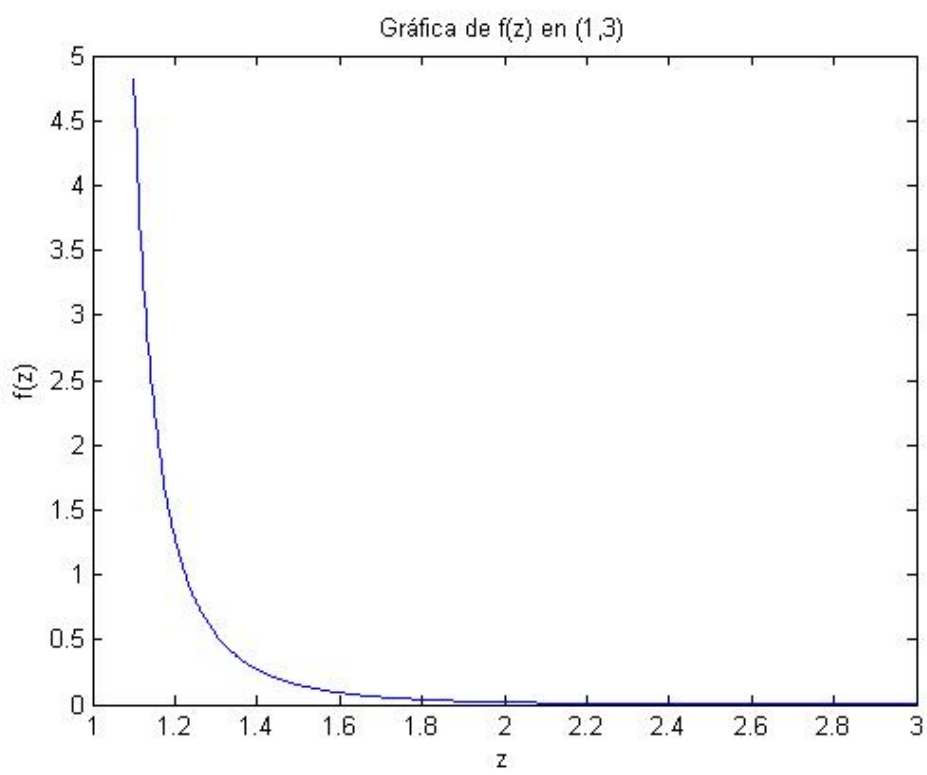

Figura 4.1: Gráfica $f(z)=\frac{8}{\sqrt{3}\left(3 z^{4}-2 z^{2}-1\right)^{3 / 2}}$ en $(1,3)$.

A continuación mostramos las condiciones sobre las masas para tener equilibrios relativos Lagrangianos del problema de tres cuerpos en $H^{2}$.

Proposición 4.1.1 En el problema de 3 cuerpos en $H^{2}$, si las partículas $m_{1}, m_{2}, m_{3}$ se encuentran inicialmente en los vértices de un triángulo equilatero, en el plano $z=$ cte $>1$, entonces existen velocidades iniciales que generan equilibrios relativos en donde el triángulo rota en su mismo plano si y solo si $m_{1}=m_{2}=m_{3}$.

\section{Demostración}

$\Rightarrow$ )

Se sigue del teorema 4.1.2.

$\Leftarrow)$

Sustituyendo en las respectivas ecuaciones de movimiento de $H^{2}$ una solución de la forma (4.4) con $i=1,2,3, \rho=\rho_{1}=\rho_{2}=\rho_{3}, z=z_{1}=z_{2}=z_{3}=\left(\rho^{2}+1\right)$ y $\alpha_{1}=0=\alpha_{2}=$ $2 \pi / 3, \alpha_{3}=4 \pi / 3$, podemos llegar al siguiente sistema

$$
\begin{aligned}
m_{1}+m_{2} & =\zeta \omega^{2}, \\
m_{2}+m_{3} & =\zeta \omega^{2}, \\
m_{3}+m_{1} & =\zeta \omega^{2},
\end{aligned}
$$


donde $\zeta=\sqrt{3}\left(3 z^{4}-2 z^{2}-1\right)^{3 / 2} / 4$. Pero para cualquier $z=$ cte $>1$ el sistema tiene solución sólo si $m_{1}=m_{2}=m_{3}=\zeta^{2} / 2$.

Tambien tenemos condiciones sobre la velodidad para tener equilibrios relativos eulerianos, la mostramos en el siguiente

Teorema 4.1.3 Consideremos el problema de 3 cuerpos en $H^{2}$ con masas iguales, $m>0$. Fijemos la masa $m_{3}$ en $(0,0,1)$ y los cuerpos con masas $m_{1}$ y $m_{2}$ en los lados opuestos de un círculo en la altura $z=$ cte $>1$. Entonces, para cada $m>0$ y $z>1$ existe un valor positivo y uno negativo de $\omega$, para los cuales se producen equilibrios relativos que rotan alrededor del eje $z$.

\section{Demostración}

Sustituyendo en las respectivas ecuaciones de movimiento una solución de la forma

$$
\begin{array}{r}
x_{1}=\rho \cos \omega t, \quad y_{1}=\rho \sin \omega t, z_{1}=z \\
x_{2}=\rho \cos (\omega t+\pi), \quad y_{1}=\rho \sin (\omega t+\pi), z_{2}=z \\
x_{3}=0, \\
y_{3}=0, \quad z_{3}=1,
\end{array}
$$

donde $\rho \geq 0$ y $z \geq 1$ son constantes que satisfacen $z^{2}=\rho^{2}+1$. Con estas expresiones podemos llegar a la siguiente

$$
\frac{4 z^{2}+1}{4 z^{3}\left(z^{2}-1\right)^{3 / 2}}=\frac{\omega^{2}}{m} .
$$

La parte izquierda de la función es positiva para $z>1$. Entonces para cada $m>0, z>1$, existe un valor positivo y uno negativo de $\omega$ los cuales generan equilibrios relativos. El signo de $\omega$ determina el sentido de rotación. 


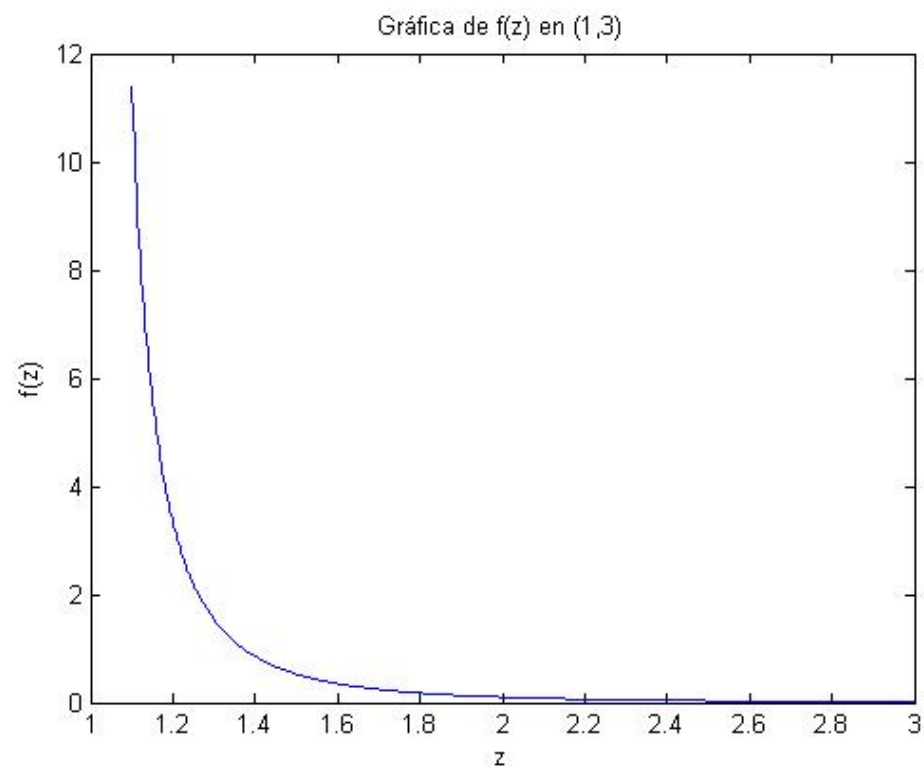

Figura 4.2: Gráfica $f(z)=\frac{4 z^{2}+1}{4 z^{3}\left(1-z^{2}\right)^{\frac{3}{2}}}$ en $(1,3)$.

\subsubsection{Equilibrios Relativos Hiperbólicos}

En esta sección mostraremos los resultados que hasta ahora se tiene sobre equilibrios relativos hiperbólicos en $H^{2}$. Iniciaremos mostrando que en el problema de $n$ cuerpos no existen equilibrios relativos hiperbólicos en el caso donde las partículas se encuentran sobre una misma geodésica

Teorema 4.1.4 A lo largo de una geódesica fija no existen equilibrios relativos hiperbólicos

\section{Demostración}

Sin pérdida de generalidad, probaremos el resultado para la geodésica que se encuentra en $x=0$. Mostraremos que las ecuaciones de movimiento en $H^{2}$ no tienen solución de la forma (4.1) con $x_{i}=0,(\rho=1), i=1,2, \cdots, n$. Sustituyendo en las ecuaciones de movimiento las siguientes expresiones

$$
x_{i}=0, \quad y_{i}=\sinh \left(\omega t+\alpha_{i}\right), \quad z_{i}=\cosh \left(\omega t+\alpha_{i}\right),
$$

la coordenada $y_{i}$ de la ecuación de movimiento del $i$-ésimo cuerpo queda como

$$
\sum_{j \neq i, j=1}^{n} \frac{m_{j}\left[\sinh \left(\omega t+\alpha_{j}\right)-\cosh \left(\alpha_{i}-\alpha_{j}\right) \sinh \left(\omega t+\alpha_{i}\right)\right]}{\left|\sinh \left(\alpha_{i}-\alpha_{j}\right)\right|^{3}}=0 .
$$


Asumamos que $\alpha_{i}>\alpha_{j}$ para $j \neq i$. Sea $\alpha_{M(i)}$ el máximo de todos los $\alpha_{j}$ con $j \neq i$. Entonces para $t \in\left(-\alpha_{M(i) / \omega},-\alpha_{i} / \omega\right)$, tenemos que $\sinh \left(\alpha t+\alpha_{j}\right)<0$ para $j \neq i$ y $\sinh \left(\alpha t+\alpha_{i}\right)>0$. Entonces la parte de la izquierda de (4.13) es negativa en este intervalo, entonces la ecuación no tiene sentido para todo $t \in \mathbb{R}$. Entonces una condición necesaria es que $\alpha_{M(i)} \geq \alpha_{i}$. Pero esta desigualdad se debe tener para todo $i=1,2, \cdots, n$. Es importante ver que esta condición nos lleva a que existe por lo menos un $i$ y un $j$ con $i \neq j$ tales que $\alpha_{i}=\alpha_{j}$. Para estos $i$ y $j$, tenemos $\sinh \left(\alpha_{i}-\alpha_{j}\right)=0$, y con este término la ecuación (4.13) no está definida. Entonces las ecuaciones de movimiento en $H^{2}$ no pueden tener soluciones de la forma (4.12). Por lo que no existen equilibrios relativos a lo largo de la geodésica $x=0$.

El siguiente resultado nos muestra la condición sobre la velocidad para generar equilibrios relativos. El teorema muestra la existencia de movimiento sobre $H^{2}$ de tres partículas que se mueven a lo largo de hipérbolas que están en planos paralelos de $\mathbb{R}^{3}$, manteniendo siempre las distancias iniciales y moviéndose sobre la misma geodésica.

Teorema 4.1.5 En el problema de tres cuerpos de masas iguales, $m=m_{1}=m_{2}=m_{3}$, en $H^{2}$, para cualquier $m>0$ y $x \neq 0$, existe un valor positivo y uno negativo de $\omega$ que generan equilibrios relativos hiperbólicos de tipo eulerianos.

\section{Demostración}

Probaremos que $q_{i}(t)=\left(x_{i}(t), y_{i}(t), z_{i}(t)\right), i=1,2,3$ es un equilibrio relativo hiperbólico para

$$
\begin{array}{r}
x_{1}=0, \quad y_{1}=\sinh \omega t, \quad z_{1}=\cosh \omega t, \\
x_{2}=x, \quad y_{1}=\rho \sinh \omega t, \quad z_{1}=\rho \cosh \omega t, \\
x_{1}=-x, \quad y_{1}=\rho \sinh \omega t, \quad z_{1}=\rho \cosh \omega t,
\end{array}
$$

donde $\rho=\left(1+x^{2}\right)^{1 / 2}$. Notemos que

$$
\begin{array}{r}
x_{1} x_{2}+y_{1} y_{2}-z_{1} z_{2}=x_{1} x_{3}+y_{1} y_{3}-z_{1} z_{3}=-\rho, \\
x_{2} x_{3}+y_{2} y_{3}-z_{2} z_{3}=-2 x^{2}-1 \\
\dot{x}_{1}^{2}+\dot{y}_{1}^{2}-\dot{z}_{1}^{2}=\omega^{2}, \quad \dot{x}_{2}^{2}+\dot{y}_{2}^{2}-\dot{z}_{2}=\dot{x}_{3}^{2}+\dot{y}_{3}^{2}-\dot{z}_{3}=\rho^{2} \omega^{2} .
\end{array}
$$

Sustituyendo estas expresiones en las ecuaciones de movimiento llegamos a

$$
\frac{4 x^{2}+1}{4 x^{2}|x|\left(x^{2}+1\right)^{3 / 2}}=\frac{\omega^{2}}{m} .
$$

El teorema queda demostrado. 


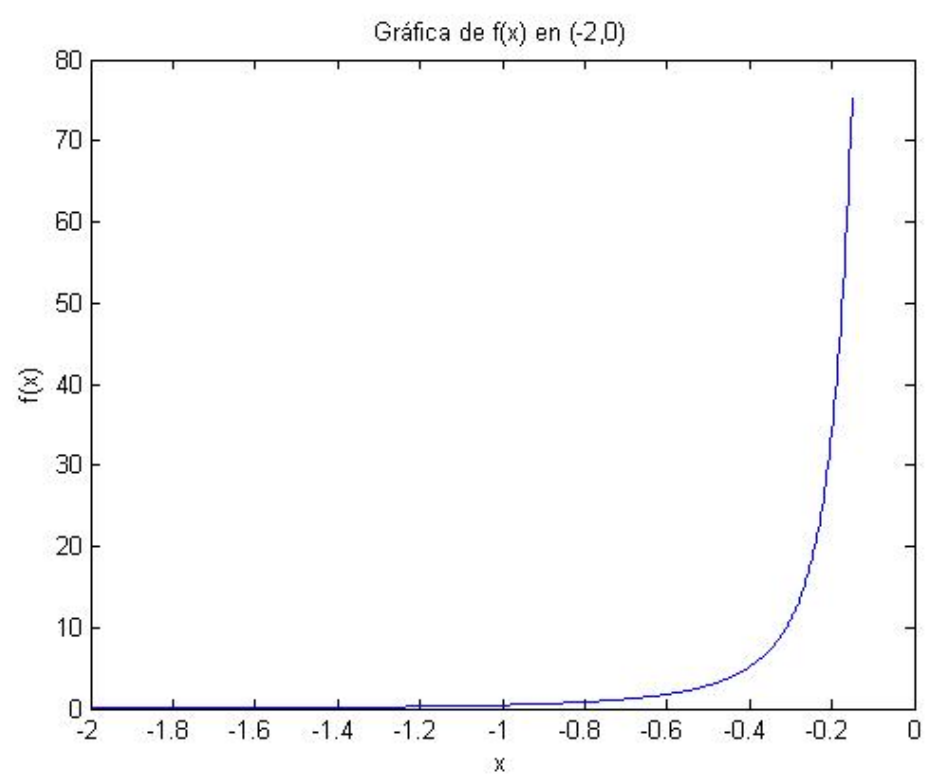

Figura 4.3: Gráfica $f(x)=\frac{4 x^{2}+1}{4 x^{3}|x|\left(1+x^{2}\right)^{\frac{3}{2}}}$ en $(-2,0)$.

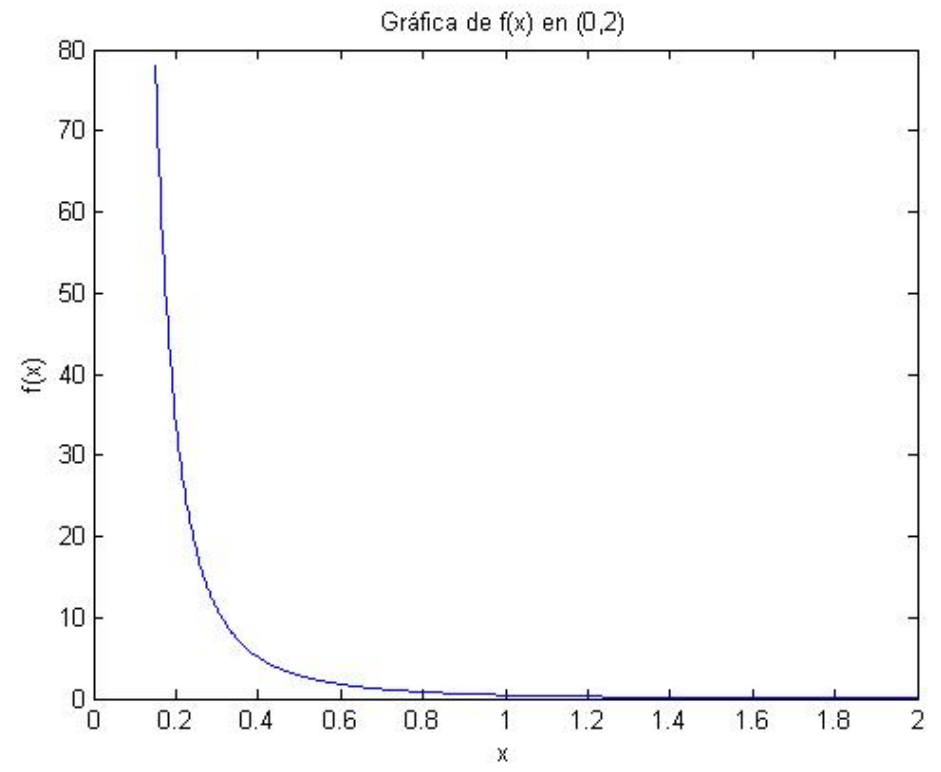

Figura 4.4: Gráfica $f(x)=\frac{4 x^{2}+1}{4 x^{2}|x|\left(1+x^{2}\right)^{\frac{3}{2}}}$ en $(0,2)$. 


\subsubsection{Equilibrios Relativos Parabólicos}

El problema de $n$ cuerpos en $H^{2}$ no tiene equilibrios relativos parabólicos, la demostración la podemos ver en el siguiente

Teorema 4.1.6 El problema de $n$ cuerpos en $\mathrm{H}^{2}$ no tiene equilibrios relativos parabólicos

\section{Demostración}

Sea $x_{i}, y_{i}, z_{i}$ como en (4.2). Entonces $\dot{x}_{i}=-b_{i}+c_{i}, \dot{y}_{i}=a_{i}+\left(c_{i}-b_{i}\right) t$ y $\dot{z}_{i}=a_{i}+\left(c_{i}-b_{i}\right) t$. La primera componente del momento angular es $\sum_{i} m_{i} a_{i}\left(b_{i}-c_{i}\right)-\sum_{i} m_{i}\left(b_{i}-c_{i}\right)^{2} t$. Como el momento angular es una primera integral, ésta debe ser constante. Entonces $b_{i}=c_{i}$. Por otra parte tenemos la condición $a_{i}^{2}+b_{i}^{2}-c_{i}^{2}=1$, entonces $a_{i}^{2}=-1$, lo que es imposible, ya que $a_{i} \in \mathbb{R}$. 


\section{Capítulo 5}

\section{Estabilidad de Equilibrios Relativos}

en $S^{2}$

En esta sección mostraremos algunos resultados acerca de la estabilidad de los equilibrios relativos elípticos eulerianos del problema de dos y tres cuerpos sobre la esfera.

Mostraremos resultados sobre estabilidad de tipo espectral, los cuales se definen de la siguiente manera

Definición 5.0.6 Un equilibrio relativo es espectralmente estable si todas las raíces del correspondiente polinomio característico $p(\lambda)=0$ satisfacen $\lambda^{2} \leq 0$.

\subsection{Problema de Dos Cuerpos}

Comencemos con el estudio del problema de dos cuerpos con masas iguales sobre $S^{2}$ con el siguiente teorema, ver figura 5.1.

Teorema 5.1.1 Consideremos órbitas periódicas del problema curvado de dos cuerpos en $S^{2}$. Si los cuerpos con masas $m_{1}=m_{2}$ se encuentran fijas en los lados opuestos de un círulo que gira uniformemente en un plano paralelo al ecuador $z=0$, entonces para $z \in(-1,1)$ las órbitas generadas son estables si $1>|z| \geq \frac{1}{\sqrt{2}}$ e inestables si $0<|z|<\frac{1}{\sqrt{2}}$. 


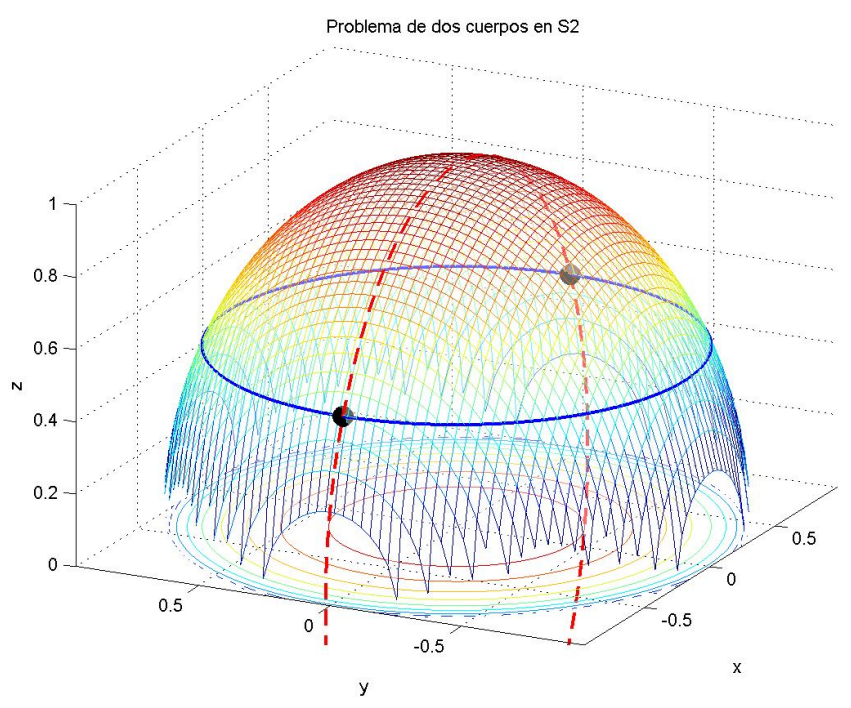

Figura 5.1: Equilibrios relativos eulerianos del problema de dos cuerpos en $S^{2}$. Dos cuerpos se encuentran girando a una altura $z$ formando una trayectoria circular (curva continua-azul) y siempre manteniendo la misma distancia, medida sobre círculos máximos (curva punteadaroja). Mostramos sólo el hemisferio norte de $S^{2}$.

\section{Demostración}

Después de normalizar las masas podemos tomar $m_{1}=m_{2}=1$. Para comenzar a analizar el problema consideremos los siguientes cambios de coordenadas que nos ayudaran a simplificar expresiones.

$$
\begin{aligned}
x_{i} & =r X_{i}, \quad y_{i}=r Y_{i}, \\
t & =r^{\frac{3}{2}} \tau, \\
Q & =(X, Y) .
\end{aligned}
$$

Hacemos tambien un reescalamiento sobre el valor de la velocidad angular, $\Omega=\frac{\omega t}{\tau}$.

De esta manera tenemos 


$$
\begin{aligned}
\Omega^{2} & =\frac{\omega^{2} t^{2}}{\tau^{2}} \\
& =\frac{\omega^{2} r^{3} \tau^{2}}{\tau^{2}} \\
& =\omega^{2} r^{3} \\
& =\frac{m_{1}|z|^{-1}+4 m_{3} z}{4 z^{2}\left(1-z^{2}\right)^{\frac{3}{2}}} r^{3} \\
& =\frac{m_{1}+4 m_{3}\left(1-r^{2}\right)}{4\left(1-r^{2}\right)^{\frac{3}{2}}} \\
& =\frac{1}{4\left(1-r^{2}\right)^{\frac{3}{2}}} .
\end{aligned}
$$

Introducimos la notación

$$
\begin{aligned}
X_{i}^{\prime}=\frac{d X_{i}}{d \tau}=\frac{d X_{i}}{d x_{i}} \frac{d x_{i}}{d \tau} & =\frac{d X_{i}}{d x i} \frac{d x_{i}}{d t} \frac{d t}{d \tau} \\
& =\frac{1}{r} \dot{x}_{i} r^{\frac{3}{2}} \\
& =r^{\frac{1}{2}} \dot{x}_{i}, \\
X_{i}^{\prime \prime}=\frac{d}{d \tau}\left(X_{i}^{\prime}\right)=\frac{d}{d \tau}\left(\dot{x}_{i} r^{\frac{1}{2}}\right) & =r^{\frac{1}{2}} \frac{d}{d \tau}\left(\dot{x}_{i}\right) \\
& =r^{\frac{1}{2} \ddot{x}_{i} r^{\frac{3}{2}}} \\
& =r^{2} \ddot{x}_{i},
\end{aligned}
$$

para $i=1,2$.

De la misma forma obtenemos las siguientes expresiones 


$$
Y_{i}^{\prime}=r^{\frac{1}{2}} \dot{y}_{i}, \quad Y_{i}^{\prime \prime}=r^{2} \ddot{y}_{i}, \quad i=1,2 .
$$

Ahora aplicamos las coordenadas reescaladas a las ecuaciones de movimiento, teniendo

$$
\begin{aligned}
\ddot{q}_{i} & =\sum_{j=1, j \neq i}^{3} \frac{m_{j}\left(q_{j}-\left(q_{i} \cdot q_{j}\right) q_{i}\right)}{\left(1-\left(q_{i} \cdot q_{j}\right)^{2}\right)^{\frac{3}{2}}}-\left(\dot{q}_{i} \cdot \dot{q}_{i}\right) q_{i} \\
& =\sum_{j=1, j \neq i}^{3} \frac{m_{j}\left(\left(x_{j}, y_{j}, z_{j}\right)-\left(\left(x_{i}, y_{i}, z_{i}\right) \cdot\left(x_{j}, y_{j}, z_{j}\right)\right)\left(x_{i}, y_{i}, z_{i}\right)\right)}{\left(1-\left(\left(x_{i}, y_{i}, z_{i}\right) \cdot\left(x_{j}, y_{j}, z_{j}\right)\right)^{2}\right)^{\frac{3}{2}}}-\left(\left(\dot{x}_{i}, \dot{y}_{i}, \dot{z}_{i}\right) \cdot\left(\dot{x}_{i}, \dot{y}_{i}, \dot{z}_{i}\right)\right)\left(x_{i}, y_{i}, z_{i}\right) \\
& =\sum_{j=1, j \neq i}^{3} \frac{m_{j}\left(\left(r X_{j}, r Y_{j}, z_{j}\right)-\left(\left(x_{i}, y_{i}, z_{i}\right) \cdot\left(x_{j}, y_{j}, z_{j}\right)\right)\left(r X_{i}, r Y_{i}, z_{i}\right)\right)}{\left(1-\left(\left(x_{i}, y_{i}, z_{i}\right) \cdot\left(x_{i}, y_{i}, z_{i}\right)\right)^{2}\right)^{\frac{3}{2}}} \\
& -\left(\left(\dot{x}_{i}, \dot{y}_{i}, \dot{z}_{i}\right) \cdot\left(\dot{x}_{i}, \dot{y}_{i}, \dot{z}_{i}\right)\right)\left(r X_{i}, r Y_{i}, z_{i}\right),
\end{aligned}
$$

para $i=1,2$.

Definimos $f_{i j}=\left(q_{i} \cdot q_{j}\right)=\left(x_{i}, y_{i}, z_{i}\right) \cdot\left(x_{i}, y_{i}, z_{i}\right)=r^{2}\left(X_{i} X_{j}+Y_{i} Y_{j}\right)+z_{i} z_{j}$. Entonces la última ecuación queda

$$
\begin{aligned}
\ddot{q}_{i} & =\sum_{j=1, j \neq i}^{3} \frac{m_{j}\left(\left(r X_{j}, r Y_{j}, z_{j}\right)-f_{i j}\left(r X_{i}, r Y_{i}, z_{i}\right)\right)}{\left(1-f_{i j}^{2}\right)^{\frac{3}{2}}}-\left(\left(\dot{x}_{i}, \dot{y}_{i}, \dot{z}_{i}\right) \cdot\left(\dot{x}_{i}, \dot{y}_{i}, \dot{z}_{i}\right)\right)\left(r X_{i}, r Y_{i}, z_{i}\right) \\
& =\sum_{j=1, j \neq i}^{3} \frac{m_{j}\left(\left(r X_{j}, r Y_{j}, z_{j}\right)-f_{i j}\left(r X_{i}, r Y_{i}, z_{i}\right)\right)}{\left(1-f_{i j}^{2}\right)^{\frac{3}{2}}}-\left(\dot{q}_{i} \cdot \dot{q}_{i}\right)\left(r X_{i}, r Y_{i}, z_{i}\right), i=1,2 .
\end{aligned}
$$

Como la coordenada $z_{i}$ del cuerpo $i$ se mantiene constante, entonces podemos ver el movimiento de cada particula sobre un plano, es decir, para el análisis podemos omitir la coordenada $z_{i}$.

$$
\ddot{q}_{i}=\sum_{j \neq i} \frac{m j\left(\left(r X_{j}, r Y_{j}\right)-f_{i j}\left(r X_{i}, r Y_{i}\right)\right)}{\left(1-f_{i j}^{2}\right)^{\frac{3}{2}}}-\left(\dot{q}_{i} \cdot \dot{q}_{i}\right)\left(r X_{i}, r Y_{i}\right), \quad i=1,2 .
$$

Ahora, tomando en cuenta que $Q_{i}^{\prime \prime}=r^{2} \ddot{q}_{i}$, tenemos

$$
\begin{aligned}
Q_{i}^{\prime \prime} & =r^{3} \sum_{j \neq i} \frac{m j\left(\left(X_{j}, Y_{j}\right)-f_{i j}\left(X_{i}, Y_{i}\right)\right)}{\left(1-f_{i j}^{2}\right)^{\frac{3}{2}}}-r^{3}\left(\dot{q}_{i} \cdot \dot{q}_{i}\right)\left(X_{i}, Y_{i}\right) \\
& =r^{3} \sum_{j \neq i} \frac{m j\left(Q_{j}-f_{i j} Q_{i}\right)}{\left(1-f_{i j}^{2}\right)^{\frac{3}{2}}}-r^{3}\left(\dot{q}_{i} \cdot \dot{q}_{i}\right) Q_{i}, \quad i=1,2 .
\end{aligned}
$$


Introducimos coordenadas rotatorias $\left(\xi_{i}, \eta_{i}\right), i=1,2$,

$$
\left(\begin{array}{c}
X_{i} \\
Y_{i}
\end{array}\right)=R(\Omega \tau)\left(\begin{array}{c}
\xi_{i} \\
\eta_{i}
\end{array}\right)
$$

donde $R(\Omega \tau)$ es la matriz de rotación

$$
R(\Omega \tau)=\left(\begin{array}{cc}
\cos \Omega \tau & -\sin \Omega \tau \\
\sin \Omega \tau & \cos \Omega \tau
\end{array}\right)
$$

Tenemos entonces

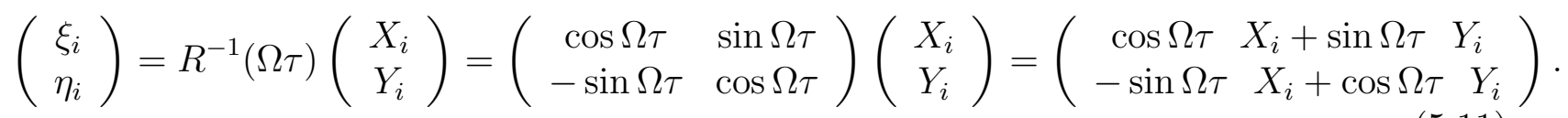

Luego

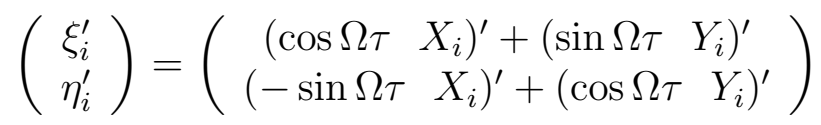

$$
\begin{aligned}
& =\left(\begin{array}{ccccc}
-\Omega \sin \Omega \tau & X_{i}+\cos \Omega \tau & X_{i}^{\prime}+\Omega \cos \Omega \tau & Y_{i}+\sin \Omega \tau & Y_{i}^{\prime} \\
-\Omega \cos \Omega \tau & X_{i}-\sin \Omega \tau & X_{i}^{\prime}-\Omega \sin \Omega \tau & Y_{i}+\cos \Omega \tau & Y_{i}^{\prime}
\end{array}\right) \\
& \left(\begin{array}{c}
\xi_{i}^{\prime \prime} \\
\eta_{i}^{\prime \prime}
\end{array}\right)=\left(\begin{array}{c}
-\Omega^{2} \cos \Omega \tau X_{i}-\Omega \sin \Omega \tau X_{i}^{\prime}-\Omega \sin \Omega \tau X_{i}^{\prime}+\cos \Omega \tau X_{i}^{\prime \prime}-\Omega^{2} \sin \Omega \tau Y_{i}+\Omega \cos \Omega \tau Y_{i}^{\prime}+ \\
\Omega \cos \Omega \tau Y_{i}^{\prime}+\sin \Omega \tau Y_{i}^{\prime \prime} \\
\Omega^{2} \sin \Omega \tau X_{i}-\Omega \cos \Omega \tau X_{i}^{\prime}-\Omega \cos \Omega \tau X_{i}^{\prime}-\sin \Omega \tau X_{i}^{\prime \prime}-\Omega^{2} \cos \Omega \tau Y_{i}-\Omega \sin \Omega \tau Y_{i}^{\prime}- \\
-\Omega \sin \Omega \tau Y_{i}^{\prime}+\cos \Omega \tau Y_{i}^{\prime \prime}
\end{array}\right) \\
& =\Omega^{2}\left(\begin{array}{c}
-\cos \Omega \tau X_{i}-\sin \Omega \tau Y_{i} \\
\sin \Omega \tau X_{i}-\cos \Omega \tau Y_{i}
\end{array}\right)+2 \Omega\left(\begin{array}{c}
-\sin \Omega \tau X_{i}^{\prime}+\cos \Omega \tau Y_{i}^{\prime} \\
-\cos \Omega \tau X_{i}^{\prime}-\sin \Omega \tau Y_{i}^{\prime}
\end{array}\right) \\
& +\left(\begin{array}{c}
\cos \Omega \tau X_{i}^{\prime \prime}+\sin \Omega \tau Y_{i}^{\prime \prime} \\
-\sin \Omega \tau X_{i}^{\prime \prime}+\cos \Omega \tau Y_{i}^{\prime \prime}
\end{array}\right) \\
& =\Omega^{2}\left(\begin{array}{c}
-\cos \Omega \tau X_{i}-\sin \Omega \tau Y_{i} \\
\sin \Omega \tau X_{i}-\cos \Omega \tau Y_{i}
\end{array}\right)+2 \Omega\left(\begin{array}{c}
-\sin \Omega \tau X_{i}^{\prime}+\cos \Omega \tau Y_{i}^{\prime} \\
-\cos \Omega \tau X_{i}^{\prime}-\sin \Omega \tau Y_{i}^{\prime}
\end{array}\right)+R^{-1}(\Omega \tau)\left(\begin{array}{c}
X_{i}^{\prime \prime} \\
Y_{i}^{\prime \prime}
\end{array}\right)
\end{aligned}
$$

Sumando y restando $2\left(\begin{array}{c}-\Omega^{2} \cos \Omega \tau X_{i}-\Omega^{2} \sin \Omega \tau Y_{i} \\ \Omega^{2} \sin \Omega \tau X_{i}-\Omega^{2} \cos \Omega \tau Y_{i}\end{array}\right)$ en la expresión anterior obtenemos 


$$
\begin{aligned}
\left(\begin{array}{c}
\xi_{i}^{\prime \prime} \\
\eta_{i}^{\prime \prime}
\end{array}\right) & =\Omega^{2}\left(\begin{array}{c}
\cos \Omega \tau X_{i}+\sin \Omega \tau Y_{i} \\
-\sin \Omega \tau X_{i}+\cos \Omega \tau Y_{i}
\end{array}\right)+2 \Omega\left(\begin{array}{c}
-\Omega \cos \Omega \tau X_{i}-\Omega \sin \Omega \tau Y_{i} \\
\Omega \sin \Omega \tau X_{i}-\Omega \cos \Omega \tau Y_{i}
\end{array}\right) \\
& +2 \Omega\left(\begin{array}{c}
-\sin \Omega \tau X_{i}^{\prime}+\cos \Omega \tau Y_{i}^{\prime} \\
-\cos \Omega \tau X_{i}^{\prime}-\sin \Omega \tau Y_{i}^{\prime}
\end{array}\right)+R^{-1}(\Omega \tau)\left(\begin{array}{c}
X_{i}^{\prime \prime} \\
Y_{i}^{\prime \prime}
\end{array}\right) \\
& =\Omega^{2}\left(\begin{array}{c}
\xi_{i} \\
\eta_{i}
\end{array}\right)+2 \Omega\left(\begin{array}{c}
\eta_{i}^{\prime} \\
-\xi_{i}^{\prime}
\end{array}\right)+R^{-1}(\Omega \tau)\left(\begin{array}{c}
X_{i}^{\prime \prime} \\
Y_{i}^{\prime \prime}
\end{array}\right) .
\end{aligned}
$$

Desarrollando los valores de $X^{\prime \prime}$ y $Y^{\prime \prime}$ en

$$
R^{-1}(\Omega \tau)\left(\begin{array}{c}
X_{i}^{\prime \prime} \\
Y_{i}^{\prime \prime}
\end{array}\right)
$$

y usando (5.8) y (5.9) obtenemos las ecuaciones de movimiento en un sistema rotatorio que gira a velocidad $\Omega$, que quedan como

$$
\begin{aligned}
\left(\begin{array}{c}
\xi_{i}^{\prime \prime} \\
\eta_{i}^{\prime \prime}
\end{array}\right) & =2 \Omega\left(\begin{array}{c}
\eta_{i}^{\prime} \\
-\xi_{i}^{\prime}
\end{array}\right)+\Omega^{2}\left(\begin{array}{c}
\xi_{i} \\
\eta_{i}
\end{array}\right)-r^{2} h_{i}\left(\begin{array}{c}
\xi_{i} \\
\eta_{i}
\end{array}\right) \\
& +m_{j}\left[\xi_{i}^{2}+\eta_{i}^{2}+\xi_{j}^{2}+\eta_{j}^{2}-2\left(\xi_{i} \xi_{j}+\eta_{i} \eta_{j}\right) \sqrt{\left(1-r^{2}\left(\xi_{i}^{2}+\eta_{i}^{2}\right)\right)\left(1-r^{2}\left(\xi_{j}^{2}+\eta_{j}^{2}\right)\right)}\right. \\
& \left.-r^{2}\left(\left(\xi_{i} \xi_{j}+\eta_{i} \eta_{j}\right)^{2}+\left(\xi_{i}^{2}+\eta_{i}^{2}\right)\left(\xi_{j}^{2}+\eta_{j}^{2}\right)\right)\right]^{-3 / 2}\left[\left(\begin{array}{c}
\xi_{j} \\
\eta_{j}
\end{array}\right)-\left(r^{2}\left(\xi_{i} \xi_{j}+\eta_{i} \eta_{j}\right)\right.\right. \\
& \left.\left.+\sqrt{\left(1-r^{2}\left(\xi_{i}^{2}+\eta_{i}^{2}\right)\right)\left(1-r^{2}\left(\xi_{j}^{2}+\eta_{j}^{2}\right)\right)}\right)\left(\begin{array}{c}
\xi_{i} \\
\eta_{i}
\end{array}\right)\right]
\end{aligned}
$$

donde

$$
h_{i}=\Omega^{2}\left(\xi_{i}^{2}+\eta_{i}^{2}\right)+2 \Omega\left(\xi_{i} \eta_{i}^{\prime}-\eta_{i} \xi_{i}^{\prime}\right)+\left(\left(\xi_{i}^{\prime 2}\right)+\left(\eta_{i}^{\prime 2}\right)\right)+\frac{r^{2}}{1-r^{2}\left(\xi_{i}^{2}+\eta_{i}^{2}\right)}\left(\xi_{i} \xi_{i}^{\prime}+\eta_{i} \eta_{i}^{\prime}\right)^{2}
$$

para $i=1,2$.

En el sistema podemos ver que un punto fijo es

$$
\xi_{1}=1, \eta_{1}=0, \xi_{2}=-1, \eta_{2}=0, \xi_{1}^{\prime}=0, \eta_{1}^{\prime},=0, \xi_{2}^{\prime}=0, \eta_{2}^{\prime}=0 .
$$

La matriz $D f$ correspondiente es una matriz de tamaño $8 \times 8$ de la forma 


$$
D f=\left(\begin{array}{ll}
0 & I \\
A & B
\end{array}\right)
$$

en donde $I$ es la matriz identidad de tamaño $4 \times 4$. La matriz $A$ en este caso tiene la forma

$$
A=\left(\begin{array}{llll}
a & 0 & b & 0 \\
0 & c & 0 & d \\
b & 0 & a & 0 \\
0 & d & 0 & c
\end{array}\right)
$$

donde

$$
\begin{aligned}
& a=\Omega^{2}-3 \Omega^{2} r^{2}-\frac{1-4 r^{2}}{\left(4-4 r^{2}\right)^{3 / 2}}+\frac{\left(6-6 r^{2}\right)\left(4-8 r^{2}\right)}{2\left(4-4 r^{2}\right)^{5 / 2}}, \\
& b=\frac{1-2 r^{2}}{\left(4-4 r^{2}\right)^{3 / 2}}-\frac{\left(6-6 r^{2}\right)\left(4-8 r^{2}\right)}{2\left(4-4 r^{2}\right)^{5 / 2}}, \\
& c=\frac{2 r^{2}-1}{\left(4-4 r^{2}\right)^{3 / 2}}+\Omega^{2}-\Omega^{2} r^{2}, \\
& d=\frac{1}{\left(4-4 r^{2}\right)^{3 / 2}} .
\end{aligned}
$$

La matriz $B$

$$
B=\left(\begin{array}{rrrr}
0 & 2 \Omega-2 \Omega r^{2} & 0 & 0 \\
-2 \Omega & 0 & 0 & 0 \\
0 & 0 & 0 & 2 \Omega-2 \Omega r^{2} \\
0 & 0 & -2 \Omega & 0
\end{array}\right)
$$

En el problema de dos cuerpos la velocidad angular debe cumplir

$$
\Omega^{2}=\frac{1}{4\left(1-r^{2}\right)^{3 / 2}}
$$

Usando (5.22) podemos reescribir a la matriz $D f$ como 


$$
\begin{aligned}
& D f=\left(\begin{array}{rrrrrrrr}
0 & 0 & 0 & 0 & 1 & 0 & 0 & 0 \\
0 & 0 & 0 & 0 & 0 & 1 & 0 & 0 \\
0 & 0 & 0 & 0 & 0 & 0 & 1 & 0 \\
0 & 0 & 0 & 0 & 0 & 0 & 0 & 1 \\
\Omega^{2}\left(2-4 r^{2}\right) & 0 & \Omega^{2}\left(-1+2 r^{2}\right) & 0 & 0 & \Omega\left(2-2 r^{2}\right) & 0 & 0 \\
0 & \frac{\Omega^{2}}{2} & 0 & \frac{\Omega^{2}}{2} & -2 \Omega & 0 & 0 & 0 \\
\Omega^{2}\left(-1+2 r^{2}\right) & 0 & \Omega^{2}\left(2-4 r^{2}\right) & 0 & 0 & 0 & 0 & \Omega\left(2-2 r^{2}\right) \\
0 & \frac{\Omega^{2}}{2} & 0 & \frac{\Omega^{2}}{2} & 0 & 0 & -2 \Omega & 0
\end{array}\right) . \\
&=\left(\begin{array}{c}
I \\
\Omega^{2} \widetilde{A} \Omega \widetilde{B}
\end{array}\right)
\end{aligned}
$$

Los eigenvalores de la matriz $D f$ son los ceros de la ecuación $\operatorname{det}(D f-\lambda I)=0$. Definamos $\mu$ de tal manera que $\lambda=\Omega \mu$, entonces buscaremos los ceros de la ecuación $\operatorname{det}(D f-\Omega \mu I)=0$.

Con la notación que hemos introducido la condición que buscamos para los valores propios puede ser escrita como

$$
\left(\begin{array}{cc}
0 & I \\
\Omega^{2} \widetilde{A} & \Omega \widetilde{B}
\end{array}\right)\left(\begin{array}{l}
u \\
v
\end{array}\right)=\Omega \mu\left(\begin{array}{l}
u \\
v
\end{array}\right),
$$

donde $u, v$ están formados por las primeras y últimas cuatro componentes, respectivamente, del eigenvector correspondiente al eigenvalor $\Omega \mu$.

Así la condición del eigenvalor con parámetro $\mu$ se sigue de

$$
v=\Omega \mu u, \quad \Omega^{2} \widetilde{A} u+\Omega \widetilde{B} \Omega u=\Omega \mu v=\Omega^{2} \mu^{2} u,
$$

diviendo entre $\Omega^{2}$, la condición debe satisfacer $D(\mu) u=0$, donde $D(\mu)=\widetilde{A}+\mu \widetilde{B}-\mu^{2} I$, y se debe tener $\operatorname{det}(D)=0$.

Denotamos a $r^{2}$ por $R$. El polinomio característico $p(\mu)$ de la matriz $\widetilde{A}+\mu \widetilde{B}-\mu^{2} I$ es

$$
p(\mu)=\mu^{8}+3 \mu^{6}-4 \mu^{4} R^{2}+3 \mu^{4}-4 \mu^{2} R^{2}+\mu^{2} .
$$

El polinomio $p(\mu)$ lo podemos escribir como

$$
p(\mu)=\mu^{2}\left(\mu^{2}+1\right)\left(\mu^{2}+2 R+1\right)\left(\mu^{2}-2 R+1\right) .
$$

Recordemos que la condición necesaria para la estabilidad espectral es que los eigenvalores de $D f$ sean iguales a cero o puramente imaginarios. 
Analicemos las raíces de $p(\mu)$ dadas por el factor $\left(\mu^{2}-2 R+1\right)=: q(\mu)$.

El factor $q(\mu)$ tiene raíces

$$
\mu= \pm \sqrt{2 R-1} .
$$

Para tener estabilidad espectral de los equilibrios relativos en el problema restringido se debe cumplir

$$
2 R-1 \leq 0,
$$

con $R \in(0,1)$.

La condición (5.29) se cumple cuando $R \in\left(0, \frac{1}{2}\right]$, es decir, cuando $z^{2} \in\left[\frac{1}{2}, 1\right)$.

Es importante señalar que este análisis es también válido para $z<0$, ya que la condición sobre la velocidad angular (5.22) es la misma para valores positivos y negativos de $z$.

\subsection{Problema de Tres Cuerpos}

Estudiaremos el problema en $S^{2}$ en donde una de las partículas se encuentra en el polo norte y las otras dos se encuentran girando en un círculo a una altura $z$, ver figura 5.2. 


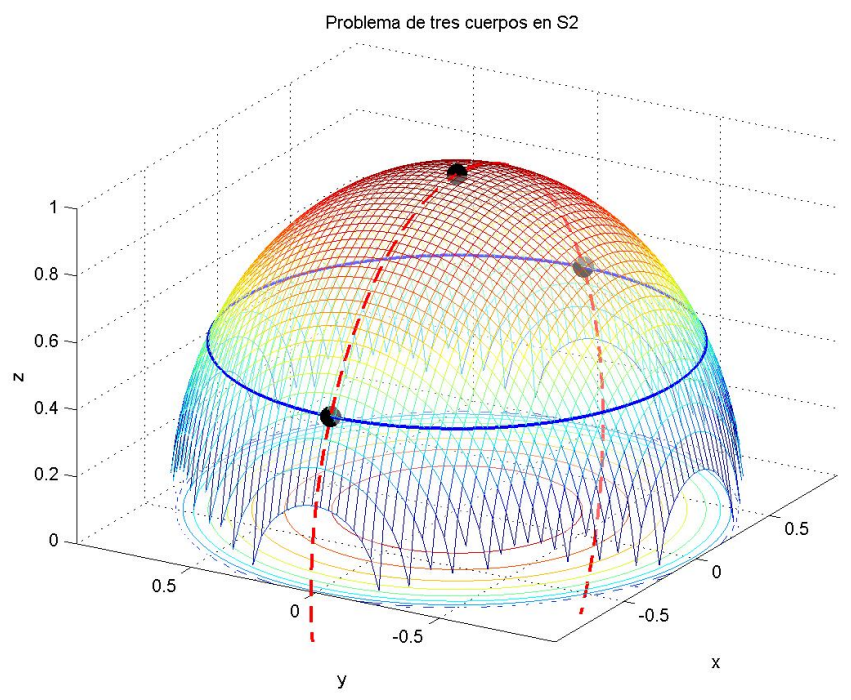

Figura 5.2: Equilibrios relativos eulerianos del problema de tres cuerpos en $S^{2}$. Una partícula se encuentra sobre el punto $(0,0,1)$ y dos cuerpos giran a una altura $z$ en trayectoria circular (curva continua-azul), manteniendo siempre la misma distancia, medida sobre geodésicas (curva punteada-roja). Mostramos sólo el hemisferio norte de $S^{2}$.

\subsubsection{Problema Restringido}

El problema restringido, como en el caso newtoniano, consiste en un sistema en donde una de las partículas tiene masa infinitesimal y la fuerza que ejerce ésta sobre las otras partículas es despreciable.

Teorema 5.2.1 Consideremos órbitas eulerianas periódicas del problema curvado de tres cuerpos en $S^{2}$ con masas $m_{1}=m_{2}$ fijas en los lados opuestos de un círculo que rota uniformemente en un plano paralelo al ecuador $z=0$, y la partícula $m_{3}$ de masa despreciable fija en el polo norte $(0,0,1)$, entonces las órbitas generadas son inestables para $z>0, y$ además este tipo de órbitas no existen si $0>z>-1$.

\section{Demostración}

Para demostrar este resultado introduciremos una partícula de masa $m_{3}=m$ fija en el punto $(0,0,1)$, y después haremos $m \rightarrow 0$, además normalizaremos el valor de las otras dos masas, es decir tomaremos $m_{1}=m_{2}=1$. Introduciremos coordenadas rotatorias para estudiar la estabilidad de equilibrios relativos, consideremos los siguientes cambios de coordenadas

$$
\begin{aligned}
x_{i} & =r X_{i}, \quad y_{i}=r Y_{i}, \\
t & =r^{\frac{3}{2}} \tau, \\
Q & =(X, Y) .
\end{aligned}
$$


Hacemos tambien un reescalamiento sobre el valor de la velocidad angular, $\Omega=\frac{\omega t}{\tau}$. Esto nos ayudará a simplificar un poco las ecuaciones.

De esta manera tenemos

$$
\begin{aligned}
\Omega^{2} & =\frac{\omega^{2} t^{2}}{\tau^{2}} \\
& =\frac{\omega^{2} r^{3} \tau^{2}}{\tau^{2}} \\
& =\omega^{2} r^{3} \\
& =\frac{m_{1}|z|^{-1}+4 m_{3} z}{4 z^{2}\left(1-z^{2}\right)^{\frac{3}{2}}} r^{3} \\
& =\frac{m_{1}+4 m_{3}\left(1-r^{2}\right)}{4\left(1-r^{2}\right)^{\frac{3}{2}}} \\
& =\frac{1+4 m_{3}\left(1-r^{2}\right)}{4\left(1-r^{2}\right)^{\frac{3}{2}}} \\
& =\frac{1+4 m\left(1-r^{2}\right)}{4\left(1-r^{2}\right)^{\frac{3}{2}}} .
\end{aligned}
$$

Introducimos la notación

$$
\begin{aligned}
X_{i}^{\prime}=\frac{d X_{i}}{d \tau}=\frac{d X_{i}}{d x_{i}} \frac{d x_{i}}{d \tau} & =\frac{d X_{i}}{d x i} \frac{d x_{i}}{d t} \frac{d t}{d \tau} \\
& =\frac{1}{r} \dot{x}_{i} r^{\frac{3}{2}} \\
& =r^{\frac{1}{2}} \dot{x}_{i},
\end{aligned}
$$




$$
\begin{aligned}
X_{i}^{\prime \prime}=\frac{d}{d \tau}\left(X_{i}^{\prime}\right)=\frac{d}{d \tau}\left(\dot{x}_{i} r^{\frac{1}{2}}\right) & =r^{\frac{1}{2}} \frac{d}{d \tau}\left(\dot{x}_{i}\right) \\
& =r^{\frac{1}{2}} \ddot{x}_{i} r^{\frac{3}{2}} \\
& =r^{2} \ddot{x}_{i}
\end{aligned}
$$

para $i=1,2,3$.

De la misma forma obtenemos las siguientes expresiones

$$
Y_{i}^{\prime}=r^{\frac{1}{2}} \dot{y}_{i}, \quad Y_{i}^{\prime \prime}=r^{2} \ddot{y}_{i}, \quad i=1,2,3 .
$$

Ahora aplicamos las coordenadas reescaladas a las ecuaciones de movimiento, teniendo

$$
\begin{aligned}
\ddot{q}_{i} & =\sum_{j=1, j \neq i}^{3} \frac{m_{j}\left(q_{j}-\left(q_{i} \cdot q_{j}\right) q_{i}\right)}{\left(1-\left(q_{i} \cdot q_{j}\right)^{2}\right)^{\frac{3}{2}}}-\left(\dot{q}_{i} \cdot \dot{q}_{i}\right) q_{i} \\
& =\sum_{j=1, j \neq i}^{3} \frac{m_{j}\left(\left(x_{j}, y_{j}, z_{j}\right)-\left(\left(x_{i}, y_{i}, z_{i}\right) \cdot\left(x_{j}, y_{j}, z_{j}\right)\right)\left(x_{i}, y_{i}, z_{i}\right)\right)}{\left(1-\left(\left(x_{i}, y_{i}, z_{i}\right) \cdot\left(x_{j}, y_{j}, z_{j}\right)\right)^{2}\right)^{\frac{3}{2}}}-\left(\left(\dot{x}_{i}, \dot{y}_{i}, \dot{z}_{i}\right) \cdot\left(\dot{x}_{i}, \dot{y}_{i}, \dot{z}_{i}\right)\right)\left(x_{i}, y_{i}, z_{i}\right) \\
& =\sum_{j=1, j \neq i}^{3} \frac{m_{j}\left(\left(r X_{j}, r Y_{j}, z_{j}\right)-\left(\left(x_{i}, y_{i}, z_{i}\right) \cdot\left(x_{j}, y_{j}, z_{j}\right)\right)\left(r X_{i}, r Y_{i}, z_{i}\right)\right)}{\left(1-\left(\left(x_{i}, y_{i}, z_{i}\right) \cdot\left(x_{i}, y_{i}, z_{i}\right)\right)^{2}\right)^{\frac{3}{2}}} \\
& -\left(\left(\dot{x}_{i}, \dot{y}_{i}, \dot{z}_{i}\right) \cdot\left(\dot{x}_{i}, \dot{y}_{i}, \dot{z}_{i}\right)\right)\left(r X_{i}, r Y_{i}, z_{i}\right),
\end{aligned}
$$

para $i=1,2,3$.

Definimos $f_{i j}=\left(q_{i} \cdot q_{j}\right)=\left(x_{i}, y_{i}, z_{i}\right) \cdot\left(x_{i}, y_{i}, z_{i}\right)=r^{2}\left(X_{i} X_{j}+Y_{i} Y_{j}\right)+z_{i} z_{j}$. Entonces la última ecuación queda

$$
\begin{aligned}
\ddot{q}_{i} & =\sum_{j=1, j \neq i}^{3} \frac{m_{j}\left(\left(r X_{j}, r Y_{j}, z_{j}\right)-f_{i j}\left(r X_{i}, r Y_{i}, z_{i}\right)\right)}{\left(1-f_{i j}^{2}\right)^{\frac{3}{2}}}-\left(\left(\dot{x}_{i}, \dot{y}_{i}, \dot{z}_{i}\right) \cdot\left(\dot{x}_{i}, \dot{y}_{i}, \dot{z}_{i}\right)\right)\left(r X_{i}, r Y_{i}, z_{i}\right) \\
& =\sum_{j=1, j \neq i}^{3} \frac{m_{j}\left(\left(r X_{j}, r Y_{j}, z_{j}\right)-f_{i j}\left(r X_{i}, r Y_{i}, z_{i}\right)\right)}{\left(1-f_{i j}^{2}\right)^{\frac{3}{2}}}-\left(\dot{q}_{i} \cdot \dot{q}_{i}\right)\left(r X_{i}, r Y_{i}, z_{i}\right), i=1,2,3 .
\end{aligned}
$$

Como la coordenada $z_{i}$ del cuerpo $i$ se mantiene constante, entonces podemos ver el movimiento de cada particula sobre un plano, es decir, para el análisis podemos omitir la coordenada $z_{i}$. 


$$
\ddot{q}_{i}=\sum_{j \neq i} \frac{m j\left(\left(r X_{j}, r Y_{j}\right)-f_{i j}\left(r X_{i}, r Y_{i}\right)\right)}{\left(1-f_{i j}^{2}\right)^{\frac{3}{2}}}-\left(\dot{q}_{i} \cdot \dot{q}_{i}\right)\left(r X_{i}, r Y_{i}\right), \quad i=1,2,3 .
$$

Ahora, tomando en cuenta que $Q_{i}^{\prime \prime}=r^{2} \ddot{q}_{i}$, tenemos

$$
\begin{aligned}
Q_{i}^{\prime \prime} & =r^{3} \sum_{j \neq i} \frac{m j\left(\left(X_{j}, Y_{j}\right)-f_{i j}\left(X_{i}, Y_{i}\right)\right)}{\left(1-f_{i j}^{2}\right)^{\frac{3}{2}}}-r^{3}\left(\dot{q}_{i} \cdot \dot{q}_{i}\right)\left(X_{i}, Y_{i}\right) \\
& =r^{3} \sum_{j \neq i} \frac{m j\left(Q_{j}-f_{i j} Q_{i}\right)}{\left(1-f_{i j}^{2}\right)^{\frac{3}{2}}}-r^{3}\left(\dot{q}_{i} \cdot \dot{q}_{i}\right) Q_{i}, \quad i=1,2,3 .
\end{aligned}
$$

Introducimos coordenadas rotatorias $\left(\xi_{i}, \eta_{i}\right), i=1,2,3$,

$$
\left(\begin{array}{c}
X_{i} \\
Y_{i}
\end{array}\right)=R(\Omega \tau)\left(\begin{array}{c}
\xi_{i} \\
\eta_{i}
\end{array}\right)
$$

en donde $R(\Omega \tau)$ es la matriz de rotación

$$
R(\Omega \tau)=\left(\begin{array}{cc}
\cos \Omega \tau & -\sin \Omega \tau \\
\sin \Omega \tau & \cos \Omega \tau
\end{array}\right)
$$

Tenemos entonces

$\left(\begin{array}{c}\xi_{i} \\ \eta_{i}\end{array}\right)=R^{-1}(\Omega \tau)\left(\begin{array}{c}X_{i} \\ Y_{i}\end{array}\right)=\left(\begin{array}{cc}\cos \Omega \tau & \sin \Omega \tau \\ -\sin \Omega \tau & \cos \Omega \tau\end{array}\right)\left(\begin{array}{c}X_{i} \\ Y_{i}\end{array}\right)=\left(\begin{array}{ccc}\cos \Omega \tau & X_{i}+\sin \Omega \tau & Y_{i} \\ -\sin \Omega \tau & X_{i}+\cos \Omega \tau & Y_{i}\end{array}\right)$.

Luego

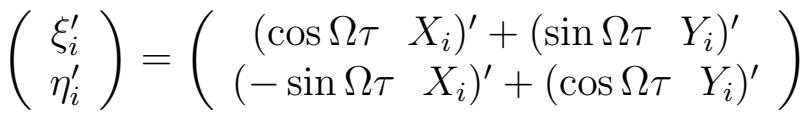

$$
\begin{aligned}
& =\left(\begin{array}{ccccc}
-\Omega \sin \Omega \tau & X_{i}+\cos \Omega \tau & X_{i}^{\prime}+\Omega \cos \Omega \tau & Y_{i}+\sin \Omega \tau & Y_{i}^{\prime} \\
-\Omega \cos \Omega \tau & X_{i}-\sin \Omega \tau & X_{i}^{\prime}-\Omega \sin \Omega \tau & Y_{i}+\cos \Omega \tau & Y_{i}^{\prime}
\end{array}\right)
\end{aligned}
$$




$$
\begin{aligned}
\left(\begin{array}{c}
\xi_{i}^{\prime \prime} \\
\eta_{i}^{\prime \prime}
\end{array}\right)= & \left(\begin{array}{cc}
-\Omega^{2} \cos \Omega \tau X_{i}-\Omega \sin \Omega \tau X_{i}^{\prime}-\Omega \sin \Omega \tau X_{i}^{\prime}+\cos \Omega \tau X_{i}^{\prime \prime}-\Omega^{2} \sin \Omega \tau Y_{i}+\Omega \cos \Omega \tau Y_{i}^{\prime}+ \\
\Omega \cos \Omega \tau Y_{i}^{\prime}+\sin \Omega \tau Y_{i}^{\prime \prime} \\
\Omega^{2} \sin \Omega \tau X_{i}-\Omega \cos \Omega \tau X_{i}^{\prime}-\Omega \cos \Omega \tau X_{i}^{\prime}-\sin \Omega \tau X_{i}^{\prime \prime}-\Omega^{2} \cos \Omega \tau Y_{i}-\Omega \sin \Omega \tau Y_{i}^{\prime}- \\
-\Omega \sin \Omega \tau Y_{i}^{\prime}+\cos \Omega \tau Y_{i}^{\prime \prime}
\end{array}\right) \\
= & \Omega^{2}\left(\begin{array}{c}
-\cos \Omega \tau X_{i}-\sin \Omega \tau Y_{i} \\
\sin \Omega \tau X_{i}-\cos \Omega \tau Y_{i}
\end{array}\right)+2 \Omega\left(\begin{array}{c}
-\sin \Omega \tau X_{i}^{\prime}+\cos \Omega \tau Y_{i}^{\prime} \\
-\cos \Omega \tau X_{i}^{\prime}-\sin \Omega \tau Y_{i}^{\prime}
\end{array}\right) \\
+ & \left(\begin{array}{c}
\cos \Omega \tau X_{i}^{\prime \prime}+\sin \Omega \tau Y_{i}^{\prime \prime} \\
-\sin \Omega \tau X_{i}^{\prime \prime}+\cos \Omega \tau Y_{i}^{\prime \prime}
\end{array}\right) \\
= & \Omega^{2}\left(\begin{array}{c}
-\cos \Omega \tau X_{i}-\sin \Omega \tau Y_{i} \\
\sin \Omega \tau X_{i}-\cos \Omega \tau Y_{i}
\end{array}\right)+2 \Omega\left(\begin{array}{c}
-\sin \Omega \tau X_{i}^{\prime}+\cos \Omega \tau Y_{i}^{\prime} \\
-\cos \Omega \tau X_{i}^{\prime}-\sin \Omega \tau Y_{i}^{\prime}
\end{array}\right)+R^{-1}(\Omega \tau)\left(\begin{array}{c}
X_{i}^{\prime \prime} \\
Y_{i}^{\prime \prime}
\end{array}\right)
\end{aligned}
$$

Sumando y restando $2\left(\begin{array}{c}-\Omega^{2} \cos \Omega \tau X_{i}-\Omega^{2} \sin \Omega \tau Y_{i} \\ \Omega^{2} \sin \Omega \tau X_{i}-\Omega^{2} \cos \Omega \tau Y_{i}\end{array}\right)$ en la expresión anterior obtenemos

$$
\begin{aligned}
\left(\begin{array}{c}
\xi_{i}^{\prime \prime} \\
\eta_{i}^{\prime \prime}
\end{array}\right) & =\Omega^{2}\left(\begin{array}{c}
\cos \Omega \tau X_{i}+\sin \Omega \tau Y_{i} \\
-\sin \Omega \tau X_{i}+\cos \Omega \tau Y_{i}
\end{array}\right)+2 \Omega\left(\begin{array}{c}
-\Omega \cos \Omega \tau X_{i}-\Omega \sin \Omega \tau Y_{i} \\
\Omega \sin \Omega \tau X_{i}-\Omega \cos \Omega \tau Y_{i}
\end{array}\right) \\
& +2 \Omega\left(\begin{array}{c}
-\sin \Omega \tau X_{i}^{\prime}+\cos \Omega \tau Y_{i}^{\prime} \\
-\cos \Omega \tau X_{i}^{\prime}-\sin \Omega \tau Y_{i}^{\prime}
\end{array}\right)+R^{-1}(\Omega \tau)\left(\begin{array}{c}
X_{i}^{\prime \prime} \\
Y_{i}^{\prime \prime}
\end{array}\right) \\
& =\Omega^{2}\left(\begin{array}{c}
\xi_{i} \\
\eta_{i}
\end{array}\right)+2 \Omega\left(\begin{array}{c}
\eta_{i}^{\prime} \\
-\xi_{i}^{\prime}
\end{array}\right)+R^{-1}(\Omega \tau)\left(\begin{array}{c}
X_{i}^{\prime \prime} \\
Y_{i}^{\prime \prime}
\end{array}\right) .
\end{aligned}
$$

Desarrollando los valores de $X^{\prime \prime}$ y $Y^{\prime \prime}$ en

$$
R^{-1}(\Omega \tau)\left(\begin{array}{c}
X_{i}^{\prime \prime} \\
Y_{i}^{\prime \prime}
\end{array}\right)
$$

y usando (5.37) y (5.38) obtenemos las ecuaciones de movimiento en un sistema rotatorio que gira a velocidad $\Omega$, que quedan como 


$$
\begin{aligned}
\left(\begin{array}{c}
\xi_{i}^{\prime \prime} \\
\eta_{i}^{\prime \prime}
\end{array}\right) & =2 \Omega\left(\begin{array}{c}
\eta_{i}^{\prime} \\
-\xi_{i}^{\prime}
\end{array}\right)+\Omega^{2}\left(\begin{array}{c}
\xi_{i} \\
\eta_{i}
\end{array}\right)-r^{2} h_{i}\left(\begin{array}{c}
\xi_{i} \\
\eta_{i}
\end{array}\right) \\
& +\sum_{j=1, j \neq i}^{3} m_{j}\left[\xi_{i}^{2}+\eta_{i}^{2}+\xi_{j}^{2}+\eta_{j}^{2}-2\left(\xi_{i} \xi_{j}+\eta_{i} \eta_{j}\right) \sqrt{\left(1-r^{2}\left(\xi_{i}^{2}+\eta_{i}^{2}\right)\right)\left(1-r^{2}\left(\xi_{j}^{2}+\eta_{j}^{2}\right)\right)}\right. \\
& \left.-r^{2}\left(\left(\xi_{i} \xi_{j}+\eta_{i} \eta_{j}\right)^{2}+\left(\xi_{i}^{2}+\eta_{i}^{2}\right)\left(\xi_{j}^{2}+\eta_{j}^{2}\right)\right)\right]^{-3 / 2}\left[\left(\begin{array}{c}
\xi_{j} \\
\eta_{j}
\end{array}\right)-\left(r^{2}\left(\xi_{i} \xi_{j}+\eta_{i} \eta_{j}\right)\right.\right. \\
& \left.\left.+\sqrt{\left(1-r^{2}\left(\xi_{i}^{2}+\eta_{i}^{2}\right)\right)\left(1-r^{2}\left(\xi_{j}^{2}+\eta_{j}^{2}\right)\right)}\right)\left(\begin{array}{c}
\xi_{i} \\
\eta_{i}
\end{array}\right)\right]
\end{aligned}
$$

donde

$$
h_{i}=\Omega^{2}\left(\xi_{i}^{2}+\eta_{i}^{2}\right)+2 \Omega\left(\xi_{i} \eta_{i}^{\prime}-\eta_{i} \xi_{i}^{\prime}\right)+\left(\left(\xi_{i}^{\prime 2}\right)+\left(\eta_{i}^{\prime 2}\right)\right)+\frac{r^{2}}{1-r^{2}\left(\xi_{i}^{2}+\eta_{i}^{2}\right)}\left(\xi_{i} \xi_{i}^{\prime}+\eta_{i} \eta_{i}^{\prime}\right)^{2}
$$

para $i=1,2,3$.

En el sistema (5.45), podemos comprobar que

$\xi_{1}=1, \eta_{1}=0, \xi_{2}=-1, \eta_{2}=0, \xi_{3}=0, \eta_{3}=0, \xi_{1}^{\prime}=0, \eta_{1}^{\prime},=0, \xi_{2}^{\prime}=0, \eta_{2}^{\prime}=0, \xi_{3}^{\prime}=0, \eta_{3}^{\prime}=0$ es punto fijo. Recordemos que $m_{1}=m_{2}=1$ y $m_{3}=m$.

Para estudiar la estabilidad espectral de este tipo de equilibrios relativos tenemos que estudiar los eigenvalores del campo vectorial de la diferencial de la función de la parte derecha de (5.45), escrito como un sistema de 12 ecuaciones evaluado en el punto fijo que exhibimos anteriormente.

La estructura de dicho campo vectorial evaluado en el punto fijo tiene la forma de la siguiente matriz cuadrada

$$
D f=\left(\begin{array}{cc}
0 & I \\
A & B
\end{array}\right)
$$

donde $I$ es la matriz identidad de tamaño $6 \times 6$, y $A$ y $B$ están dadas de la siguiente forma 


$$
\begin{aligned}
& B=\left(\begin{array}{cccccc}
0 & 2 \Omega-2 \Omega r^{2} & 0 & 0 & 0 & 0 \\
-2 \Omega & 0 & 0 & 0 & 0 & 0 \\
0 & 0 & 0 & 2 \Omega-2 \Omega r^{2} & 0 & 0 \\
0 & 0 & -2 \Omega^{2} & 0 & 0 & 0 \\
0 & 0 & 0 & 0 & 0 & 2 \Omega \\
0 & 0 & 0 & 0 & -2 \Omega & 0
\end{array}\right) \\
& A=\left(\begin{array}{cccccc}
a & 0 & b & 0 & e & 0 \\
0 & c & 0 & d & 0 & 1 \\
b & 0 & a & 0 & e & 0 \\
0 & d & 0 & c & 0 & 1 \\
-2 & 0 & -2 & 0 & f & 0 \\
0 & 1 & 0 & 1 & 0 & g
\end{array}\right)
\end{aligned}
$$

donde $a, b, c, d, e, f, g$ están dados por los siguientes valores

$$
\begin{aligned}
& a=\Omega^{2}-3 \Omega^{2} r^{2}+\frac{m\left(2-r^{2}\right)}{\sqrt{1-r^{2}}}-\frac{1-4 r^{2}}{\left(4-4 r^{2}\right)^{3 / 2}}+\frac{\left(6-6 r^{2}\right)\left(4-r^{2}\right)}{2\left(4-4 r^{2}\right)^{5 / 2}} \\
& b=-\frac{2 r^{2}-1}{\left(4-4 r^{2}\right)^{\frac{3}{2}}}-\frac{\left(6 r^{2}-6\right)\left(8 r^{2}-4\right)}{2\left(4-4 r^{2}\right)^{\frac{5}{2}}}, \\
& c=\Omega^{2}+\frac{\left(2 r^{2}-1\right)}{8\left(1-r^{2}\right)^{\frac{3}{2}}}-\Omega^{2} r^{2}-m\left(\sqrt{1-r^{2}}\right), \\
& d=\frac{1}{\left(4-4 r^{2}\right)^{\frac{3}{2}}}, \\
& e=2 m\left(r^{2}-1\right), \\
& f=\Omega^{2}+4 \sqrt{1-r^{2}} \\
& g=\Omega^{2}-2 \sqrt{1-r^{2}} .
\end{aligned}
$$

Recordemos que para la existencia de equilibrios relativos la velocidad angular debe cumplir

$$
\Omega^{2}=\frac{1+4 m\left(1-r^{2}\right)}{4\left(1-r^{2}\right)^{\frac{3}{2}}} .
$$


A la matriz $D f$ la podemos reescribir como

$$
D f=\left(\begin{array}{cc}
0 & I \\
A & \Omega \widetilde{B}
\end{array}\right)
$$

con

$$
\widetilde{B}=\left(\begin{array}{cccccc}
0 & 2-2 r^{2} & 0 & 0 & 0 & 0 \\
-2 & 0 & 0 & 0 & 0 & 0 \\
0 & 0 & 0 & 2-2 r^{2} & 0 & 0 \\
0 & 0 & -2 & 0 & 0 & 0 \\
0 & 0 & 0 & 0 & 0 & 2 \\
0 & 0 & 0 & 0 & -2 & 0
\end{array}\right)
$$

Los vectores propios de $D f$ son los ceros de la ecuación $\operatorname{det}(D f-\lambda I)=0$. Definamos $\mu$ tal que $\lambda=\Omega \mu$, por lo que buscaremos los ceros de la ecuación $\operatorname{det}(D f-\Omega \mu I)=0$. La condicón que buscaremos para los valores propios puede ser escrita como

$$
\left(\begin{array}{cc}
0 & I \\
A & \Omega \widetilde{B}
\end{array}\right)\left(\begin{array}{l}
u \\
v
\end{array}\right)=\Omega \mu\left(\begin{array}{l}
u \\
v
\end{array}\right)
$$

donde $u, v$ están formados por las primeras y últimas cuatro componentes, respectivamente, del eigenvector correspondiente al eigenvalor $\Omega \mu$.

Así la condición del eigenvalor con parámetro $\mu$ se sigue de

$$
v=\Omega \mu u, \quad A u+\Omega \widetilde{B} \Omega \mu u=\Omega \mu v=\Omega^{2} \mu^{2} u .
$$

Denotemos al polinomio caracteristico como $p(\mu)$. La condición de valor propio debe satisfacer

$$
p(\mu)=\operatorname{det}\left(A+\Omega^{2} \mu \widetilde{B}-\Omega^{2} \mu^{2}\right)=0 .
$$

Si denotamos a $r^{2}$ por $R$ entonces

$$
A+\Omega^{2} \mu \widehat{B}-\Omega^{2} \mu^{2}=\left(\begin{array}{cccccc}
f_{1} & g_{1} & g_{2} & 0 & g_{7} & 0 \\
g_{3} & f_{2} & 0 & g_{5} & 0 & m \\
g_{2} & 0 & f_{1} & g_{4} & g_{7} & 0 \\
0 & g_{5} & g_{6} & f_{2} & 0 & m \\
-2 & 0 & -2 & 0 & f_{3} & -g_{6} \\
0 & 1 & 0 & 1 & g_{6} & f_{4}
\end{array}\right),
$$

donde 


$$
\begin{aligned}
& f_{1}=\frac{4 m-4 R m+1}{4(1-R)^{\frac{3}{2}}}+\frac{4 R-1}{8(1-R)^{\frac{3}{2}}}-\frac{6 R-3}{8(1-R)^{\frac{3}{2}}}-\frac{3 R(4 m-4 R m+1)}{4(1-R)^{\frac{3}{2}}} \\
& -\frac{\mu^{2}(4 m-4 R m+1)}{4(1-R)^{\frac{3}{2}}}-\frac{m(R-2)}{\sqrt{1-R}}, \\
& f_{2}=\frac{4 m-4 R m+1}{4(1-R)^{\frac{3}{2}}}-m \sqrt{1-R}+\frac{2 R-1}{8(1-R)^{\frac{3}{2}}}-\frac{R(4 m-4 R m+1)}{4(1-R)^{\frac{3}{2}}}-\frac{\mu^{2}(4 m-4 R m+1)}{4(1-R)^{\frac{3}{2}}}, \\
& f_{3}=\frac{4 m-4 R m+1}{4(1-R)^{\frac{3}{2}}}+4 \sqrt{1-R}-\frac{\mu^{2}(4 m-4 R m+1)}{4(1-R)^{\frac{3}{2}}}, \\
& f_{4}=\frac{4 m-4 R m+1}{4(1-R)^{\frac{3}{2}}}-2 \sqrt{1-R}-\frac{\mu^{2}(4 m-4 R m+1)}{4(1-R)^{\frac{3}{2}}}, \\
& g_{1}=-\frac{\mu(2 R-2)(4 m-4 R m+1)}{4(1-R)^{\frac{3}{2}}}, \\
& g_{2}=\frac{6 R-3}{8(1-R)^{\frac{3}{2}}}-\frac{2 R-1}{8(1-R)^{\frac{3}{2}}}, \\
& g_{3}=-\frac{\mu(8 m-8 R m+2)}{4(1-R)^{\frac{3}{2}}}, \\
& g_{4}=-\frac{\mu(2 R-2)(4 m-4 R m+1)}{4(1-R)^{\frac{3}{2}}}, \\
& g_{5}=\frac{1}{8(1-R)^{\frac{3}{2}}}, \\
& g_{6}-\frac{\mu(8 m-8 R m+2)}{4(1-R)^{\frac{3}{2}}}, \\
& g_{7}=m(2 R-2) .
\end{aligned}
$$


El determinante de la matriz (5.57) es

$$
\begin{aligned}
& p(\mu)=\frac{-1}{(4096)(R-1)^{9}} \cdot \mu^{2} \cdot\left(\mu^{2}+1\right) \cdot(4 R m-4 m-1)^{3} \cdot(4 R m-4 m-2 R \\
& \left.-4 \mu^{2} m-\mu^{2}+4 \mu^{2} R m-1\right) \cdot\left(16 \mu^{6} R^{2} m^{2}-32 \mu^{6} R m^{2}-8 \mu^{6} R m+16 \mu^{6} m^{2}\right. \\
& +8 \mu^{6} m+\mu^{6}+32 \mu^{4} R^{3} m+32 \mu^{4} R^{2} m^{2}-88 \mu^{4} R^{2} m-8 \mu^{4} R^{2}-64 \mu^{4} R m^{2} \\
& +68 \mu^{4} R m+14 \mu^{4} R+32 \mu^{4} m^{2}-12 \mu^{4} m-5 \mu^{4}-128 \mu^{2} R^{4} m-128 \mu^{2} R^{4} \\
& +512 \mu^{2} R^{3} m+528 \mu^{2} R^{3}+16 \mu^{2} R^{2} m^{2}-744 \mu^{2} R^{2} m-800 \mu^{2} R^{2}-32 \mu^{2} R m^{2} \\
& +464 \mu^{2} R m+524 \mu^{2} R+16 \mu^{2} m^{2}-104 \mu^{2} m-125 \mu^{2}+256 R^{5}-128 R^{4} m-1152 R^{4} \\
& \left.+480 R^{3} m+2032 R^{3}-656 R^{2} m-1752 R^{2}+388 R m+734 R-84 m-119\right)
\end{aligned}
$$

Sin embargo, cuando tomamos $m \rightarrow 0$ obtenemos

$$
\begin{aligned}
& p(\mu)=\frac{1}{4096(R-1)^{9}} \mu^{2}\left(\mu^{2}+1\right)\left(\mu^{2}+2 R+1\right)\left(-\mu^{6}+8 \mu^{4} R^{2}-14 \mu^{4} R+5 \mu^{4}\right. \\
& +128 \mu^{2} R^{4}-528 \mu^{2} R^{3}+800 \mu^{2} R^{2}-524 \mu^{2} R+125 \mu^{2}-256 R^{5}+1152 R^{4} \\
& \left.-2032 R^{3}+1752 R^{2}-734 R+119\right) \\
& =\frac{-1}{4096(R-1)^{9}} \mu^{2}\left(\mu^{2}+1\right)\left(\mu^{2}+2 R+1\right)\left(\mu^{2}-2 R+1\right)\left(\mu^{4}-8 \mu^{2} R^{2}+16 \mu^{2} R\right. \\
& \left.-6 \mu^{2}-128 R^{4}+512 R^{3}-760 R^{2}+496 R-119\right) .
\end{aligned}
$$

El factor $\mu$ en $p(\mu)$ aparece en potencias pares, entonces hacemos el cambio de variable $M=\mu^{2}$, para obtener el polinomio $\widehat{p}(M)$, entonces para tener estabilidad del equilibrio relativo necesitamos raíces reales negativas de $\widehat{p}(M)$.

$$
\begin{aligned}
& \widehat{p}(\mu)=\frac{-1}{4096(R-1)^{9}} M(M+1)(M+2 R+1)(M-2 R+1)\left(M^{2}-8 M R^{2}+16 M R\right. \\
& \left.-6 M-128 R^{4}+512 R^{3}-760 R^{2}+496 R-119\right)
\end{aligned}
$$

La última ecuación la podemos escribir como

$$
\widehat{p}(\mu)=\frac{-1}{4096(R-1)^{9}} M(M+1)(M+2 R+1)(M-2 R+1) q_{1}(M) q_{2}(M)
$$

donde 


$$
\begin{aligned}
q_{1}(M)= & M-2 R+1 \\
q_{2}(M)= & M^{2}-8 M R^{2}+16 M R-6 M-128 R^{4}+512 R^{3} \\
& -760 R^{2}+496 R-119 \\
= & M^{2}+M\left(-8 R^{2}+16 R-6\right)-128 R^{4}+512 R^{3}-760 R^{2}+496 R-119 \\
= & M^{2}+M h_{1}(R)+h_{2}(R) .
\end{aligned}
$$

El factor $q_{1}$ nos exhibe raíces reales negativas si $R<1 / 2$, así que para analizar $q_{2}$ nos enfocaremos en valores de $R$ en el intervalo $(0,1 / 2)$, y utilizaremos la regla de los signos de Descartes. Notemos que $h_{1}(R)$ es negativo para valores en $(0,1 / 2)$, el factor $h_{2}(R)$ tiene raíces $\frac{\sqrt{2}}{4}+1 \approx 1.3536,1-\frac{\sqrt{2}}{4} \approx 0.6464,1+\frac{i}{4}, 1-\frac{i}{4}$, ver figuras (5.3) y (5.4). Conociendo estas raíces y notando que $h_{2}(0)<0$ tenemos que el factor $q_{2}(M)$ tiene un cambio de signo, es decir una raíz real positiva si $R \in(0,1 / 2)$. Luego el polinomio $\widehat{p}$ tiene siempre una raíz real positiva y las órbitas generadas son inestables.

Notemos que si $z<0$ la ecuación (5.30) se convierte en

$$
\frac{1-4 m\left(1-r^{2}\right)}{4\left(1-r^{2}\right)^{3 / 2}}
$$

expresión válida si el numerador es positivo, es decir si $1-4 m\left(1-r^{2}\right)>0$, condición que se cumple si $r^{2}>1-\frac{1}{4 m}>0$ y que no tiene ningún sentido en nuestro problema si $m \rightarrow 0$.

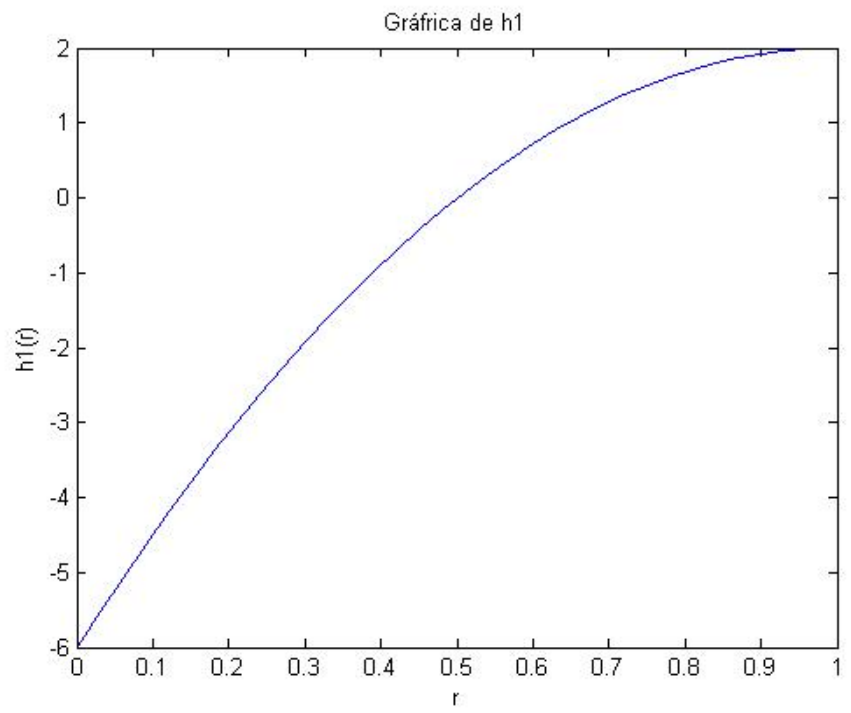

Figura 5.3: Gráfica de $h_{1}(R)$. 


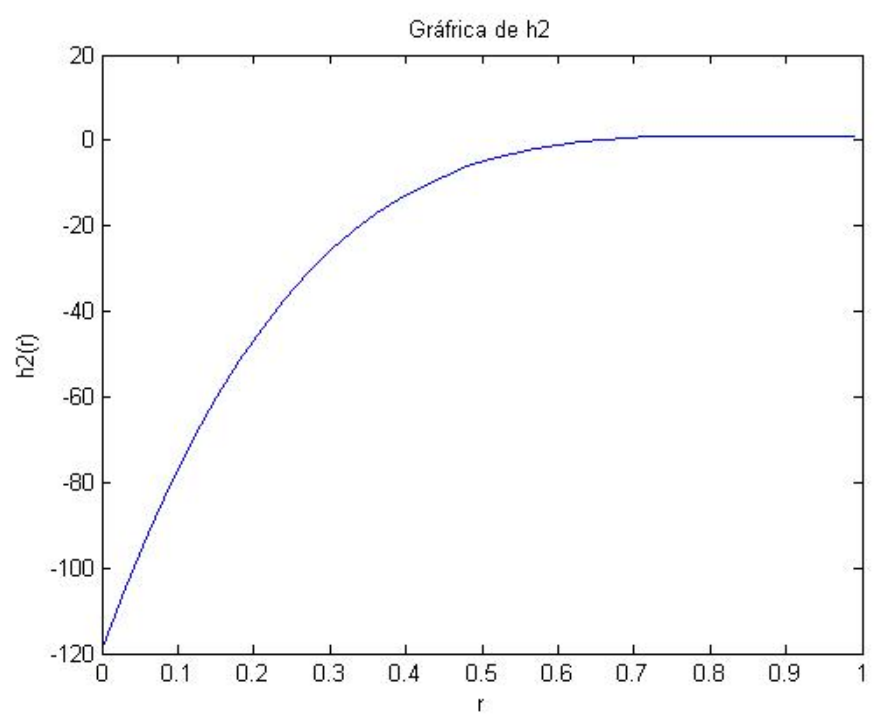

Figura 5.4: Gráfica de $h_{2}(R)$.

OBSERVACIÓN 1 Es claro que el problema restringido se convierte en un problema de dos cuerpos con masas iguales, sin embargo en los teoremas (5.1.1) y (5.2.1) obtenemos diferentes resultados, esto se debe la diferencia del espacio fase en que se exponga el problema. Primero vimos, en el teorema (5.1.1), que si consideramos el problema en donde las masas iguales giran uniformemente de forma paralela al plano xy entonces existe un intervalo sobre el eje $z$ en donde las órbitas son estables, y un intervalo en donde son inestables; por otra parte si consideramos, como lo marca el teorema (5.2.1), el problema de dos cuerpos en donde las partículas de masas iguales giran de forma paralela al eje xy y además existe otra partícula de masa $m$ fija en el polo norte de las esfera, si tomamos el límite $m \rightarrow 0$, entonces las órbitas generadas son inestables.

La razón por la que tenemos diferentes resultados es que el problema está planteado en distintos contextos. Cuando consideramos sólo dos partículas, como en el primer caso, el problema está formulado en el espacio fase

$$
\left(\mathbb{R}^{2 n} \backslash \triangle\right) \times T \mathbb{R}^{2 n} \subseteq \mathbb{R}^{8},
$$

donde $T \mathbb{R}^{2 n}$ es el haz tangente de $\mathbb{R}^{2 n},(n=2)$.

Si consideramos tres partículas, como en el segundo caso (una de masa m en el polo norte) el problema está planteado en el espacio fase

$$
\left(\mathbb{R}^{2 n} \backslash \triangle\right) \times T \mathbb{R}^{2 n} \subseteq \mathbb{R}^{12},
$$


donde $T \mathbb{R}^{2 n}$ es el haz tangente de $\mathbb{R}^{2 n},(n=3)$.

En ambos casos $\triangle$ está dado por las correspondientes singularidades, explicadas en la sección (2.5).

Dicho en otras palabras, los resultados sobre estabilidad son diferentes ya que las partículas, en ambos planteamientos, tienen diferente libertad de movimiento debido a que los espacios en donde se encuentran son diferentes.

\subsubsection{Masas Iguales}

Teorema 5.2.2 Consideremos órbitas eulerianas periódicas del problema curvado de tres cuerpos en $S^{2}$, con masas $m_{1}=m_{2}=m_{3}$, en donde $m_{1}$ y $m_{2}$ se encuentran fijas en los lados opuestos de un círculo que rota uniformemente en un plano paralelo al ecuador $z=0$, y la partícula $m_{3}$ fija en el polo norte $(0,0,1)$, entonces para $z \in(-1 / 2,0) \cup(0,1)$ las órbitas generadas son inestables, y el movimiento no es posible si $z \in(-1,-1 / 2)$. Notemos que en $z=0$ tenemos una singularidad.

\section{Demostración}

Para estudiar la estabilidad de equilibrios relativos introduciremos coordenadas rotatorias. Consideremos los siguientes cambios de coordenadas y reescalamiento de tiempo, este último para simplificar el valor de $\omega$.

$$
\begin{aligned}
x_{i} & =r X_{i}, \quad y_{i}=r Y_{i}, \\
t & =r^{\frac{3}{2}} \tau, \\
Q & =(X, Y) .
\end{aligned}
$$

Hacemos tambien un reescalamiento sobre el valor de la velocidad angular, $\Omega=\frac{\omega t}{\tau}$. De esta manera tenemos 


$$
\begin{aligned}
\Omega^{2} & =\frac{\omega^{2} t^{2}}{\tau^{2}} \\
& =\frac{\omega^{2} r^{3} \tau^{2}}{\tau^{2}} \\
& =\omega^{2} r^{3} \\
& =\frac{m_{3}|z|^{-1}+4 m_{1} z}{4 z^{2}\left(1-z^{2}\right)^{\frac{3}{2}}} r^{3} \\
& =\frac{m_{3}+4 m_{1}\left(1-r^{2}\right)}{4\left(1-r^{2}\right)^{\frac{3}{2}}} .
\end{aligned}
$$

Introducimos la notación

$$
\begin{gathered}
X_{i}^{\prime}=\frac{d X_{i}}{d \tau}=\frac{d X_{i}}{d x_{i}} \frac{d x_{i}}{d \tau}=\frac{d X_{i}}{d x i} \frac{d x_{i}}{d t} \frac{d t}{d \tau} \\
=\frac{1}{r} \dot{x}_{i} r^{\frac{3}{2}} \\
=r^{\frac{1}{2}} \dot{x}_{i}, \\
X_{i}^{\prime \prime}=\frac{d}{d \tau}\left(X_{i}^{\prime}\right)=\frac{d}{d \tau}\left(\dot{x}_{i} r^{\frac{1}{2}}\right)=r^{\frac{1}{2}} \frac{d}{d \tau}\left(\dot{x}_{i}\right) \\
=r^{\frac{1}{2}} \ddot{x}_{i} r^{\frac{3}{2}} \\
=r^{2} \ddot{x}_{i},
\end{gathered}
$$

para $i=1,2,3$.

De la misma forma obtenemos las siguientes expresiones

$$
Y_{i}^{\prime}=r^{\frac{1}{2}} \dot{y}_{i}, \quad Y_{i}^{\prime \prime}=r^{2} \ddot{y}_{i}, \quad i=1,2,3 .
$$

Ahora aplicamos las coordenadas reescaladas a las ecuaciones de movimiento, teniendo 


$$
\begin{aligned}
\ddot{q}_{i} & =\sum_{j=1, j \neq i}^{3} \frac{m_{j}\left(q_{j}-\left(q_{i} \cdot q_{j}\right) q_{i}\right)}{\left(1-\left(q_{i} \cdot q_{j}\right)^{2}\right)^{\frac{3}{2}}}-\left(\dot{q}_{i} \cdot \dot{q}_{i}\right) q_{i} \\
& =\sum_{j=1, j \neq i}^{3} \frac{m_{j}\left(\left(x_{j}, y_{j}, z_{j}\right)-\left(\left(x_{i}, y_{i}, z_{i}\right) \cdot\left(x_{j}, y_{j}, z_{j}\right)\right)\left(x_{i}, y_{i}, z_{i}\right)\right)}{\left(1-\left(\left(x_{i}, y_{i}, z_{i}\right) \cdot\left(x_{j}, y_{j}, z_{j}\right)\right)^{2}\right)^{\frac{3}{2}}}-\left(\left(\dot{x}_{i}, \dot{y}_{i}, \dot{z}_{i}\right) \cdot\left(\dot{x}_{i}, \dot{y}_{i}, \dot{z}_{i}\right)\right)\left(x_{i}, y_{i}, z_{i}\right) \\
& =\sum_{j=1, j \neq i}^{3} \frac{m_{j}\left(\left(r X_{j}, r Y_{j}, z_{j}\right)-\left(\left(x_{i}, y_{i}, z_{i}\right) \cdot\left(x_{j}, y_{j}, z_{j}\right)\right)\left(r X_{i}, r Y_{i}, z_{i}\right)\right)}{\left(1-\left(\left(x_{i}, y_{i}, z_{i}\right) \cdot\left(x_{i}, y_{i}, z_{i}\right)\right)^{2}\right)^{\frac{3}{2}}} \\
& -\left(\left(\dot{x}_{i}, \dot{y}_{i}, \dot{z}_{i}\right) \cdot\left(\dot{x}_{i}, \dot{y}_{i}, \dot{z}_{i}\right)\right)\left(r X_{i}, r Y_{i}, z_{i}\right),
\end{aligned}
$$

para $i=1,2,3$.

Definimos $f_{i j}=\left(q_{i} \cdot q_{j}\right)=\left(x_{i}, y_{i}, z_{i}\right) \cdot\left(x_{i}, y_{i}, z_{i}\right)=r^{2}\left(X_{i} X_{j}+Y_{i} Y_{j}\right)+z_{i} z_{j}$. Entonces la última ecuación queda

$$
\begin{aligned}
\ddot{q}_{i} & =\sum_{j=1, j \neq i}^{3} \frac{m j\left(\left(r X_{j}, r Y_{j}, z_{j}\right)-f_{i j}\left(r X_{i}, r Y_{i}, z_{i}\right)\right)}{\left(1-f_{i j}^{2}\right)^{\frac{3}{2}}}-\left(\left(\dot{x}_{i}, \dot{y}_{i}, \dot{z}_{i}\right) \cdot\left(\dot{x}_{i}, \dot{y}_{i}, \dot{z}_{i}\right)\right)\left(r X_{i}, r Y_{i}, z_{i}\right) \\
& =\sum_{j=1, j \neq i}^{3} \frac{m j\left(\left(r X_{j}, r Y_{j}, z_{j}\right)-f_{i j}\left(r X_{i}, r Y_{i}, z_{i}\right)\right)}{\left(1-f_{i j}^{2}\right)^{\frac{3}{2}}}-\left(\dot{q}_{i} \cdot \dot{q}_{i}\right)\left(r X_{i}, r Y_{i}, z_{i}\right), i=1,2,3 .
\end{aligned}
$$

Como la coordenada $z_{i}$ del cuerpo $i$ se mantiene constante, entonces podemos ver el movimiento de cada particula sobre un plano, es decir, para el análisis podemos omitir la coordenada $z_{i}$.

$$
\ddot{q}_{i}=\sum_{j \neq i} \frac{m j\left(\left(r X_{j}, r Y_{j}\right)-f_{i j}\left(r X_{i}, r Y_{i}\right)\right)}{\left(1-f_{i j}^{2}\right)^{\frac{3}{2}}}-\left(\dot{q}_{i} \cdot \dot{q}_{i}\right)\left(r X_{i}, r Y_{i}\right), \quad i=1,2,3
$$

Ahora, tomando en cuenta que $Q_{i}^{\prime \prime}=r^{2} \ddot{q}_{i}$, tenemos

$$
\begin{aligned}
Q_{i}^{\prime \prime} & =r^{3} \sum_{j \neq i} \frac{m j\left(\left(X_{j}, Y_{j}\right)-f_{i j}\left(X_{i}, Y_{i}\right)\right)}{\left(1-f_{i j}^{2}\right)^{\frac{3}{2}}}-r^{3}\left(\dot{q}_{i} \cdot \dot{q}_{i}\right)\left(X_{i}, Y_{i}\right) \\
& =r^{3} \sum_{j \neq i} \frac{m j\left(Q_{j}-f_{i j} Q_{i}\right)}{\left(1-f_{i j}^{2}\right)^{\frac{3}{2}}}-r^{3}\left(\dot{q}_{i} \cdot \dot{q}_{i}\right) Q_{i}, \quad i=1,2,3 .
\end{aligned}
$$


Introducimos coordenadas rotatorias $\left(\xi_{i}, \eta_{i}\right), i=1,2,3$,

$$
\left(\begin{array}{c}
X_{i} \\
Y_{i}
\end{array}\right)=R(\Omega \tau)\left(\begin{array}{c}
\xi_{i} \\
\eta_{i}
\end{array}\right)
$$

en donde $R(\Omega \tau)$ es la matriz de rotación

$$
R(\Omega \tau)=\left(\begin{array}{cc}
\cos \Omega \tau & -\sin \Omega \tau \\
\sin \Omega \tau & \cos \Omega \tau
\end{array}\right)
$$

Tenemos entonces

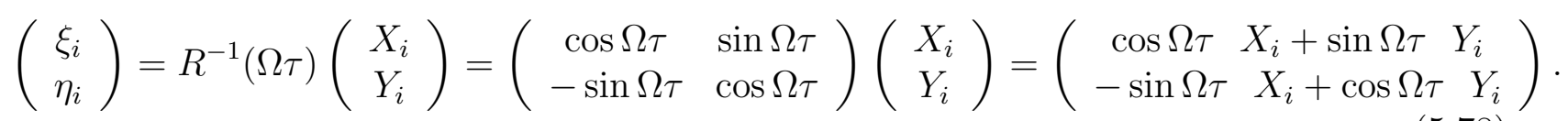

Luego

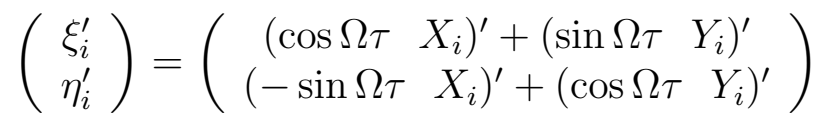

$$
\begin{aligned}
& =\left(\begin{array}{ccccc}
-\Omega \sin \Omega \tau & X_{i}+\cos \Omega \tau & X_{i}^{\prime}+\Omega \cos \Omega \tau & Y_{i}+\sin \Omega \tau & Y_{i}^{\prime} \\
-\Omega \cos \Omega \tau & X_{i}-\sin \Omega \tau & X_{i}^{\prime}-\Omega \sin \Omega \tau & Y_{i}+\cos \Omega \tau & Y_{i}^{\prime}
\end{array}\right) \\
& \left(\begin{array}{c}
\xi_{i}^{\prime \prime} \\
\eta_{i}^{\prime \prime}
\end{array}\right)=\left(\begin{array}{c}
-\Omega^{2} \cos \Omega \tau X_{i}-\Omega \sin \Omega \tau X_{i}^{\prime}-\Omega \sin \Omega \tau X_{i}^{\prime}+\cos \Omega \tau X_{i}^{\prime \prime}-\Omega^{2} \sin \Omega \tau Y_{i}+\Omega \cos \Omega \tau Y_{i}^{\prime}+ \\
\Omega \cos \Omega \tau Y_{i}^{\prime}+\sin \Omega \tau Y_{i}^{\prime \prime} \\
\Omega^{2} \sin \Omega \tau X_{i}-\Omega \cos \Omega \tau X_{i}^{\prime}-\Omega \cos \Omega \tau X_{i}^{\prime}-\sin \Omega \tau X_{i}^{\prime \prime}-\Omega^{2} \cos \Omega \tau Y_{i}-\Omega \sin \Omega \tau Y_{i}^{\prime}- \\
-\Omega \sin \Omega \tau Y_{i}^{\prime}+\cos \Omega \tau Y_{i}^{\prime \prime}
\end{array}\right) \\
& =\Omega^{2}\left(\begin{array}{c}
-\cos \Omega \tau X_{i}-\sin \Omega \tau Y_{i} \\
\sin \Omega \tau X_{i}-\cos \Omega \tau Y_{i}
\end{array}\right)+2 \Omega\left(\begin{array}{c}
-\sin \Omega \tau X_{i}^{\prime}+\cos \Omega \tau Y_{i}^{\prime} \\
-\cos \Omega \tau X_{i}^{\prime}-\sin \Omega \tau Y_{i}^{\prime}
\end{array}\right) \\
& +\left(\begin{array}{c}
\cos \Omega \tau X_{i}^{\prime \prime}+\sin \Omega \tau Y_{i}^{\prime \prime} \\
-\sin \Omega \tau X_{i}^{\prime \prime}+\cos \Omega \tau Y_{i}^{\prime \prime}
\end{array}\right) \\
& =\Omega^{2}\left(\begin{array}{c}
-\cos \Omega \tau X_{i}-\sin \Omega \tau Y_{i} \\
\sin \Omega \tau X_{i}-\cos \Omega \tau Y_{i}
\end{array}\right)+2 \Omega\left(\begin{array}{c}
-\sin \Omega \tau X_{i}^{\prime}+\cos \Omega \tau Y_{i}^{\prime} \\
-\cos \Omega \tau X_{i}^{\prime}-\sin \Omega \tau Y_{i}^{\prime}
\end{array}\right)+R^{-1}(\Omega \tau)\left(\begin{array}{c}
X_{i}^{\prime \prime} \\
Y_{i}^{\prime \prime}
\end{array}\right)
\end{aligned}
$$

Sumando y restando $2\left(\begin{array}{c}-\Omega^{2} \cos \Omega \tau X_{i}-\Omega^{2} \sin \Omega \tau Y_{i} \\ \Omega^{2} \sin \Omega \tau X_{i}-\Omega^{2} \cos \Omega \tau Y_{i}\end{array}\right)$ en la expresión anterior obtenemos 


$$
\begin{aligned}
\left(\begin{array}{c}
\xi_{i}^{\prime \prime} \\
\eta_{i}^{\prime \prime}
\end{array}\right) & =\Omega^{2}\left(\begin{array}{c}
\cos \Omega \tau X_{i}+\sin \Omega \tau Y_{i} \\
-\sin \Omega \tau X_{i}+\cos \Omega \tau Y_{i}
\end{array}\right)+2 \Omega\left(\begin{array}{c}
-\Omega \cos \Omega \tau X_{i}-\Omega \sin \Omega \tau Y_{i} \\
\Omega \sin \Omega \tau X_{i}-\Omega \cos \Omega \tau Y_{i}
\end{array}\right) \\
& +2 \Omega\left(\begin{array}{c}
-\sin \Omega \tau X_{i}^{\prime}+\cos \Omega \tau Y_{i}^{\prime} \\
-\cos \Omega \tau X_{i}^{\prime}-\sin \Omega \tau Y_{i}^{\prime}
\end{array}\right)+R^{-1}(\Omega \tau)\left(\begin{array}{c}
X_{i}^{\prime \prime} \\
Y_{i}^{\prime \prime}
\end{array}\right) \\
& =\Omega^{2}\left(\begin{array}{c}
\xi_{i} \\
\eta_{i}
\end{array}\right)+2 \Omega\left(\begin{array}{c}
\eta_{i}^{\prime} \\
-\xi_{i}^{\prime}
\end{array}\right)+R^{-1}(\Omega \tau)\left(\begin{array}{c}
X_{i}^{\prime \prime} \\
Y_{i}^{\prime \prime}
\end{array}\right) .
\end{aligned}
$$

Desarrollando los valores de $X^{\prime \prime}$ y $Y^{\prime \prime}$ en

$$
R^{-1}(\Omega \tau)\left(\begin{array}{c}
X_{i}^{\prime \prime} \\
Y_{i}^{\prime \prime}
\end{array}\right)
$$

y usando (5.75) y (5.76) obtenemos las ecuaciones de movimiento en un sistema rotatorio que gira a velocidad $\Omega$, que quedan como

$$
\begin{aligned}
\left(\begin{array}{c}
\xi_{i}^{\prime \prime} \\
\eta_{i}^{\prime \prime}
\end{array}\right) & =2 \Omega\left(\begin{array}{c}
\eta_{i}^{\prime} \\
-\xi_{i}^{\prime}
\end{array}\right)+\Omega^{2}\left(\begin{array}{c}
\xi_{i} \\
\eta_{i}
\end{array}\right)-r^{2} h_{i}\left(\begin{array}{c}
\xi_{i} \\
\eta_{i}
\end{array}\right) \\
& +\sum_{j=1, j \neq i}^{3} m_{j}\left[\xi_{i}^{2}+\eta_{i}^{2}+\xi_{j}^{2}+\eta_{j}^{2}-2\left(\xi_{i} \xi_{j}+\eta_{i} \eta_{j}\right) \sqrt{\left(1-r^{2}\left(\xi_{i}^{2}+\eta_{i}^{2}\right)\right)\left(1-r^{2}\left(\xi_{j}^{2}+\eta_{j}^{2}\right)\right)}\right. \\
& \left.-r^{2}\left(\left(\xi_{i} \xi_{j}+\eta_{i} \eta_{j}\right)^{2}+\left(\xi_{i}^{2}+\eta_{i}^{2}\right)\left(\xi_{j}^{2}+\eta_{j}^{2}\right)\right)\right]^{-3 / 2}\left[\left(\begin{array}{c}
\xi_{j} \\
\eta_{j}
\end{array}\right)-\left(r^{2}\left(\xi_{i} \xi_{j}+\eta_{i} \eta_{j}\right)\right.\right. \\
& \left.\left.+\sqrt{\left(1-r^{2}\left(\xi_{i}^{2}+\eta_{i}^{2}\right)\right)\left(1-r^{2}\left(\xi_{j}^{2}+\eta_{j}^{2}\right)\right)}\right)\left(\begin{array}{c}
\xi_{i} \\
\eta_{i}
\end{array}\right)\right]
\end{aligned}
$$

donde

$$
h_{i}=\Omega^{2}\left(\xi_{i}^{2}+\eta_{i}^{2}\right)+2 \Omega\left(\xi_{i} \eta_{i}^{\prime}-\eta_{i} \xi_{i}^{\prime}\right)+\left(\left(\xi_{i}^{\prime 2}\right)+\left(\eta_{i}^{\prime 2}\right)\right)+\frac{r^{2}}{1-r^{2}\left(\xi_{i}^{2}+\eta_{i}^{2}\right)}\left(\xi_{i} \xi_{i}^{\prime}+\eta_{i} \eta_{i}^{\prime}\right)^{2},
$$

para $i=1,2,3$.

En el sistema (5.83), podemos comprobar que

$\xi_{1}=1, \eta_{1}=0, \xi_{2}=-1, \eta_{2}=0, \xi_{3}=0, \eta_{3}=0, \xi_{1}^{\prime}=0, \eta_{1}^{\prime},=0, \xi_{2}^{\prime}=0, \eta_{2}^{\prime}=0, \xi_{3}^{\prime}=0, \eta_{3}^{\prime}=0$ 
es punto fijo. De acuerdo con estos índices los cuerpos tienen masas $m_{1}=m_{2}=m$ y $m_{3}=M$.

Para estudiar la estabilidad espectral de este tipo de equilibrios relativos tenemos que estudiar los eigenvalores del campo vectorial de la diferencial de la función de la parte derecha de (5.83), escrito como un sistema de 12 ecuaciones evaluado en el punto fijo que exhibimos anteriormente.

La estructura de dicho campo vectorial evaluado en el punto fijo tiene la forma de la siguiente matriz cuadrada

$$
D f=\left(\begin{array}{cc}
0 & I \\
A & B
\end{array}\right),
$$

donde $I$ es la matriz identidad de tamaño $6 \times 6$, y $A$ y $B$ están dadas de la siguiente forma

$$
B=\left(\begin{array}{cccccc}
0 & 2 \Omega-2 \Omega r^{2} & 0 & 0 & 0 & 0 \\
-2 \Omega & 0 & 0 & 0 & 0 & 0 \\
0 & 0 & 0 & 2 \Omega-2 \Omega r^{2} & 0 & 0 \\
0 & 0 & -2 \Omega^{2} & 0 & 0 & 0 \\
0 & 0 & 0 & 0 & 0 & 2 \\
0 & 0 & 0 & 0 & -2 \Omega & 0
\end{array}\right),
$$

$$
A=\left(\begin{array}{cccccc}
a & 0 & b & 0 & e & 0 \\
0 & c & 0 & d & 0 & 1 \\
b & 0 & a & 0 & e & 0 \\
0 & d & 0 & c & 0 & 1 \\
-2 & 0 & -2 & 0 & f & 0 \\
0 & 1 & 0 & 1 & 0 & g
\end{array}\right)
$$

donde $a, b, c, d, e, f, g$ están dados por los siguientes valores 
$a=\Omega^{2}+\frac{12 m}{\left(4-4 r^{2}\right)^{\frac{5}{2}}}-3 \Omega^{2} r^{2}+\frac{m\left(4 r^{2}-1\right)}{\left(4-4 r^{2}\right)^{\frac{3}{2}}}-\frac{36 m r^{2}}{\left(4-4 r^{2}\right)^{\frac{5}{2}}}+\frac{24 m r^{4}}{\left(4-4 r^{2}\right)^{\frac{5}{2}}}+\frac{M r^{2}}{(2 M+1) \sqrt{1-r^{2}}}$,

$b=-\frac{2 r^{2}-1}{\left(4-4 r^{2}\right)^{\frac{3}{2}}}-\frac{\left(6 r^{2}-6\right)\left(8 r^{2}-4\right)}{2\left(2-4 r^{2}\right)^{\frac{5}{2}}}$,

$c=2 M\left(r^{2}-1\right)$,

$d=\Omega^{2}+\frac{m\left(2 r^{2}-1\right)}{8\left(1-r^{2}\right)^{\frac{3}{2}}}-\Omega^{2} r^{2}-M\left(\sqrt{1-r^{2}}\right)$,

$e=\frac{1}{\left(4-4 r^{2}\right)^{\frac{3}{2}}}$

$f=\Omega^{2}+4 m \sqrt{1-r^{2}}$,

$g=\Omega^{2}-2 m \sqrt{1-r^{2}}+m$.

Recordemos que para la existencia de equilibrios relativos la velocidad angular debe cumplir

$$
\Omega^{2}=\frac{m+4 M\left(1-r^{2}\right)}{4\left(1-r^{2}\right)^{\frac{3}{2}}}
$$

A la matriz $D f$ la podemos reescribir como

$$
D f=\left(\begin{array}{cc}
0 & I \\
A & \Omega \widetilde{B}
\end{array}\right)
$$

con

$$
\widetilde{B}=\left(\begin{array}{cccccc}
0 & 2-2 r^{2} & 0 & 0 & 0 & 0 \\
-2 & 0 & 0 & 0 & 0 & 0 \\
0 & 0 & 0 & 2-2 r^{2} & 0 & 0 \\
0 & 0 & -2 & 0 & 0 & 0 \\
0 & 0 & 0 & 0 & 0 & 2 \\
0 & 0 & 0 & 0 & -2 & 0
\end{array}\right)
$$


Los vectores propios de $D f$ son los ceros de la ecuación $\operatorname{det}(D f-\lambda I)=0$. Definamos $\mu$ tal que $\lambda=\Omega \mu$, por lo que buscaremos los ceros de la ecuación $\operatorname{det}(D f-\Omega \mu I)=0$. La condicón que buscaremos para los valores propios puede ser escrita como

$$
\left(\begin{array}{cc}
0 & I \\
A & \Omega \widetilde{B}
\end{array}\right)\left(\begin{array}{l}
u \\
v
\end{array}\right)=\Omega \mu\left(\begin{array}{l}
u \\
v
\end{array}\right),
$$

donde $u, v$ están formados por las primeras y últimas cuatro componentes, respectivamente, del eigenvector correspondiente al eigenvalor $\Omega \mu$.

Así la condición del eigenvalor con parámetro $\mu$ se sigue de

$$
v=\Omega \mu u, \quad A u+\Omega \widetilde{B} \Omega \mu u=\Omega \mu v=\Omega^{2} \mu^{2} u .
$$

Denotemos al polinomio caracteristico como $p(\mu)$. La condición de valor propio debe satisfacer

$$
p(\mu)=\operatorname{det}\left(A+\Omega^{2} \mu \widetilde{B}-\Omega^{2} \mu^{2}\right)=0 .
$$

Si denotamos a $r^{2}$ por $R$ entonces

$$
A+\Omega^{2} \mu \widehat{B}-\Omega^{2} \mu^{2}=
$$

$$
\left(\begin{array}{cccccc}
f_{1} & \frac{\mu(2 R-2)(4 R-5)}{4(1-R)^{\frac{3}{2}}} & \frac{6 R-3}{8(1-R)^{\frac{3}{2}}}-\frac{2 R-1}{8(1-R)^{\frac{3}{2}}} & 0 & 2 R-2 & 0 \\
\frac{\mu(8 R-10)}{4(1-R)^{\frac{3}{2}}} & f_{2} & 0 & \frac{1}{8(1-R)^{\frac{3}{2}}} & 0 & 1 \\
\frac{6 R-3}{8(1-R)^{\frac{3}{2}}-\frac{2 R-1}{8(1-R)^{\frac{3}{2}}}} & 0 & f_{1} & \frac{\mu(2 R-2)(4 R-5)}{4(1-R)^{\frac{3}{2}}} & 2 R-2 & 0 \\
0 & \frac{1}{8(1-R)^{\frac{3}{2}}} & \frac{\mu(8 R-10)}{4(1-R)^{\frac{3}{2}}} & f_{2} & 0 & 1 \\
-2 & 0 & -2 & 0 & f_{3} & -\frac{\mu(8 R-10)}{4(1-R)^{\frac{3}{2}}} \\
0 & 1 & 0 & 1 & \frac{\mu(8 R-10)}{4(1-R)^{\frac{3}{2}}} & f_{4}
\end{array}\right),
$$

donde 


$$
\begin{aligned}
& f_{1}=\frac{4 R-1}{8(1-R)^{\frac{3}{2}}}-\frac{R-2}{\sqrt{1-R}}-\frac{4 R-5}{4(1-R)^{\frac{3}{2}}}-\frac{6 R-3}{8(1-R)^{\frac{3}{2}}}+\frac{3 R(4 R-5)}{4(1-R)^{\frac{3}{2}}}+\frac{\mu^{2}(4 R-5)}{4(1-R)^{\frac{3}{2}}} \\
& f_{2}=\frac{2 R-1}{8(1-R)^{\frac{3}{2}}}-\sqrt{1-R}-\frac{4 R-5}{4(1-R)^{\frac{3}{2}}}+\frac{R(4 R-5)}{4(1-R)^{\frac{3}{2}}}+\frac{\mu^{2}(4 R-5)}{4(1-R)^{\frac{3}{2}}}, \\
& f_{3}=4 \sqrt{1-R}-\frac{4 R-5}{4(1-R)^{\frac{3}{2}}}+\frac{\mu^{2}(4 R-5)}{4(1-R)^{\frac{3}{2}}} \\
& f_{4}=\frac{\mu^{2}(4 R-5)}{4(1-R)^{\frac{3}{2}}}-\frac{4 R-5}{4(1-R)^{\frac{3}{2}}}-2 \sqrt{1-R} .
\end{aligned}
$$

El factor $\mu$ en $p(\mu)$ aparece en potencias pares, entonces hacemos el cambio de variable $M=\mu^{2}$, para obtener el polinomio $\widehat{p}(M)$, entonces para tener estabilidad del equilibrio relativo necesitamos raíces reales negativas de $\widehat{p}(M)$. Después de multiplicar al polinomio $\widehat{p}(M)$ por el factor $-4096(1-R)^{9} /(4 R-5)^{3}$ obtenemos

$$
\widehat{p}(M)=M(M+1) Q_{1}(M) Q_{2}(M),
$$

donde

$$
\begin{aligned}
Q_{1}(M)= & 2 R+4 M R-5 M-5, \\
Q_{2}(M)= & 16 M^{3} R^{2}-40 M^{3} R+25 M^{3}+32 M^{2} R^{3}-64 M^{2} R^{2}+18 M^{2} R+15 M^{2} \\
& -256 M R^{4}+1040 M R^{3}-1528 M R^{2}+956 M R-213 M+256 R^{5} \\
& -1280 R^{4}+2512 R^{3}-2408 R^{2}+1122 R-203 \\
= & \left(16 R^{2}-40 R+25\right) M^{3} \\
& +\left(32 R^{3}-64 R^{2}+18 R+15\right) M^{2} \\
& +\left(-256 R^{4}+1040 R^{3}-1528 R^{2}+956 M-213\right) M \\
& +256 R^{5}-1280 R^{4}+2512 R^{3}-2408 R^{2}+1122 R-203 .
\end{aligned}
$$

El factor $Q_{1}(M)$ tiene raíz $M=\frac{5-2 R}{4 R-5}<0$, si $R \in(0,1)$. Analizaremos las raíces del polinomio $Q_{2}(M)$, reescribiéndolo

$$
Q_{2}(M)=g_{1}(R) M^{3}+g_{2}(R) M^{2}+g_{3}(R) M+g_{4}(R) .
$$

El factor $g_{1}(R)$ es positivo, excepto en la raíz doble $R=\frac{40}{32}>1$. El factor $g_{2}(R)$ tiene puntos críticos en $R_{1}=\frac{128+\sqrt{9472}}{192} \approx 1.173563544191518$ y en $R_{2}=\frac{128-\sqrt{9472}}{192} \approx$ 0.159769789141815 , donde $R_{2}$ es un máximo local. Tenemos $g_{2}(0), g_{2}(1), g_{2}\left(R_{2}\right)>0$ por lo que $g_{2}(R)>0$ para $R \in(0,1)$. Para analizar el comportamiento de $g_{4}(R)$ revisamos su derivada, $g_{4}^{\prime}(R)$, que tiene raíces $R_{1}=1.221606070873750+0.083431707913399 i, R_{2}=$ 
$1.221606070873750-0.083431707913399 i, R_{3}=0.924141480011802, R_{4}=0.632646378240695$. Las raíces reales de $g_{4}^{\prime}(R)$ nos muestran el mínimo y el máximo local de $g_{4}(R)$. Notemos que $g_{4}(0), g_{4}\left(R_{3}\right), g_{4}\left(R_{4}\right), g_{4}(1)<0$, por lo que $g_{4}(R)<0$ para $R \in(0,1)$, ver figuras (5.5), (5.6) $\mathrm{y}(5.7)$.

Hemos analizado el signo de los coeficientes de $Q_{2}(R)$, mostrando que, independientemente del signo del coeficiente $g_{3}(R)$, hay una variación de signo de los coeficientes de $Q_{2}(R)$. Por la regla de los signos de Descartes el polinomio $Q_{2}(R)$ tiene una raíz real positiva, $M_{0}$, misma que es ríz de $\widehat{p}(M)$. La raíz $M_{0}$ de $\widehat{p}(M)$ genera dos raíces reales de $p(\mu)$, con lo que mostramos que existen dos valores propios reales de la matriz $D f$. Así los equilibrios relativos generados son espectralmente inestables.

Lo que hemos mostrado hasta aquí es el caso donde la partícula $m_{1}$ y la partícula $m_{2}$ se encuentran en el hemisferio norte de la esfera. Mostraremos lo que sucede cuando éstas se encuentran en el hemisferio sur

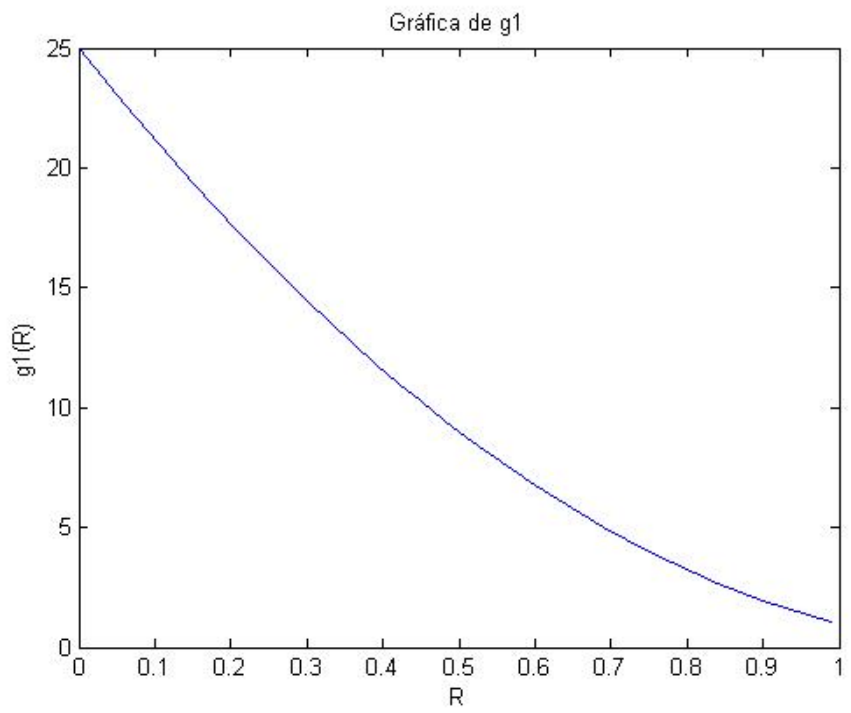

Figura 5.5: Gráfica de $g_{1}(R)$. 


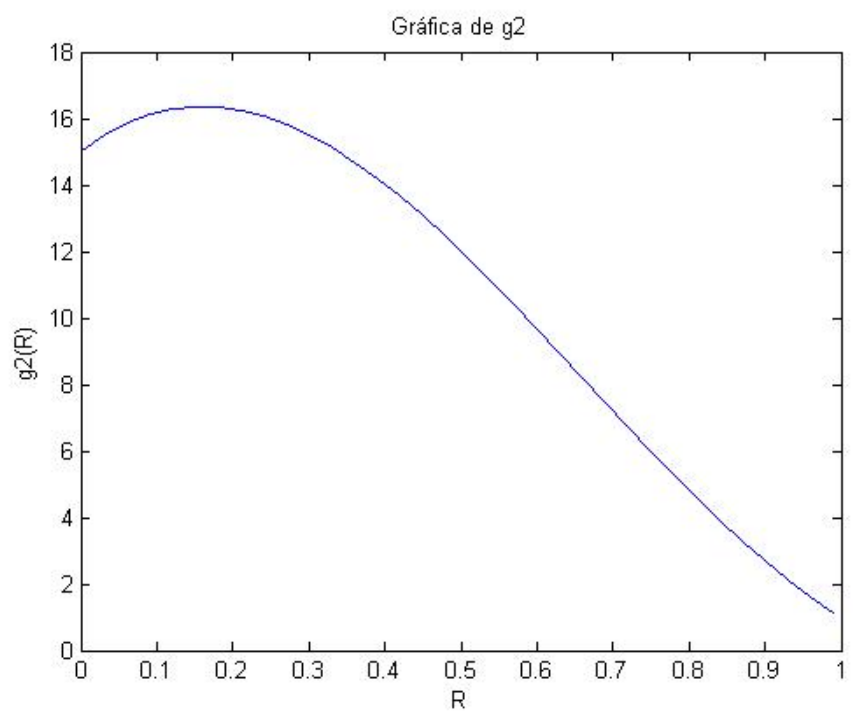

Figura 5.6: Gráfica de $g_{2}(R)$.

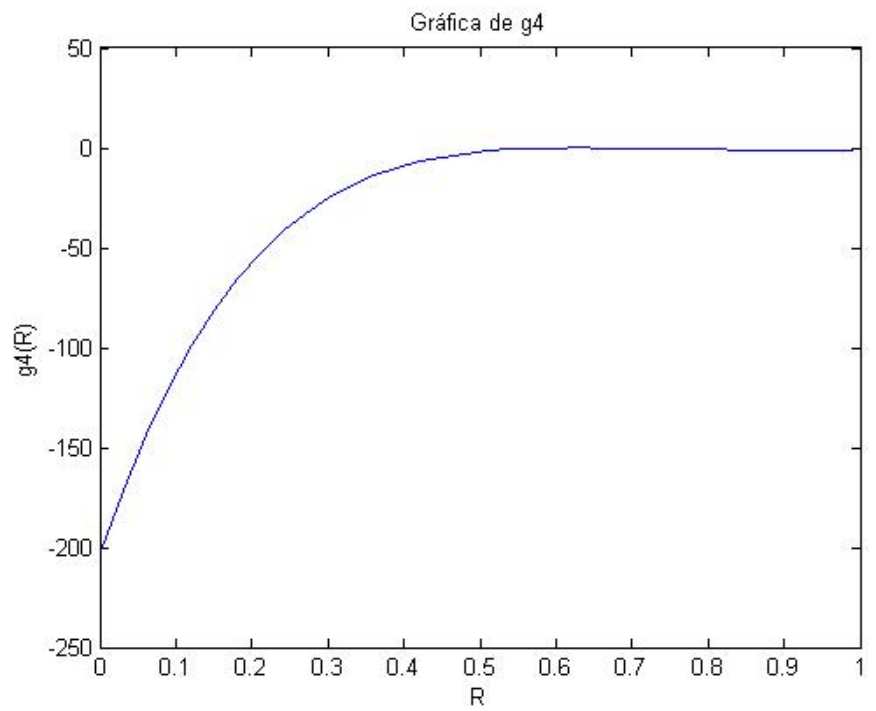

Figura 5.7: Gráfica de $g_{4}(R)$.

A continuación mostramos el análisis para el caso donde $z_{1}, z_{2}<0$, en este caso notemos que $z_{1} z_{3}<0, z_{2} z_{3}<0$ y $z_{1} z_{2}>0$ en los correspondientes términos de la ecuación (5.83). Además la condición (3.39) para $z<0$ en términos de $r$ y masas iguales a uno queda como

$$
\omega^{2}=\frac{1-4\left(1-r^{2}\right)}{4\left(1-r^{2}\right)^{3 / 2}} .
$$


Notemos que la ecuación (5.100) tiene sentido sólo si el numerador es positivo, es decir si $r^{2}>3 / 4$.

La matriz $D f$ queda como

$$
D f=\left(\begin{array}{cc}
0 & I \\
A & \Omega \widetilde{B}
\end{array}\right)
$$

con

$$
\begin{gathered}
\widetilde{B}=\left(\begin{array}{cccccc}
0 & 2-2 r^{2} & 0 & 0 & 0 & 0 \\
-2 & 0 & 0 & 0 & 0 & 0 \\
0 & 0 & 0 & 2-2 r^{2} & 0 & 0 \\
0 & 0 & -2 & 0 & 0 & 0 \\
0 & 0 & 0 & 0 & 0 & 2 \\
0 & 0 & 0 & 0 & -2 & 0
\end{array}\right), \\
A=\left(\begin{array}{cccccc}
a & 0 & b & 0 & e & 0 \\
0 & c & 0 & d & 0 & 1 \\
b & 0 & a & 0 & e & 0 \\
0 & d & 0 & c & 0 & 1 \\
-2 & 0 & -2 & 0 & f & 0 \\
0 & 1 & 0 & 1 & 0 & g
\end{array}\right)
\end{gathered}
$$

donde

$$
\begin{aligned}
a & =\Omega-\frac{1-4 r^{2}}{8\left(1-r^{2}\right)^{3 / 2}}+\frac{r^{2}-2}{\left(1-r^{2}\right)^{1 / 2}}+\frac{3\left(1-2 r^{2}\right)}{8\left(1-r^{2}\right)^{3 / 2}}-3 \Omega r^{2}, \\
b & =\frac{1-2 r^{2}}{8\left(1-r^{2}\right)^{3 / 2}}-\frac{3\left(1-2 r^{2}\right)}{\left(1-r^{2}\right)^{3 / 2}} \\
c & =\frac{2 r^{2}-1}{8\left(1-r^{2}\right)^{3 / 2}}+\Omega^{2}-\Omega^{2} r^{2}+\left(1-r^{2}\right)^{1 / 2} \\
d & =\frac{1}{8\left(1-r^{2}\right)^{3 / 2}} \\
e & =2 r^{2}-2 \\
f & =\Omega^{2}-4\left(1-r^{2}\right)^{1 / 2} \\
g & =\Omega^{2}+2\left(1-r^{2}\right)^{1 / 2}
\end{aligned}
$$

Al igual que en el caso de $z_{1}, z_{2}, z_{3}>0$, tenemos que la condición para encontrar los valores propios es resolver la ecuación

$$
p(\mu)=\operatorname{det}\left(A+\Omega^{2} \mu \widehat{B}-\Omega^{2} \mu^{2}\right)=0 .
$$

Si denotamos a $r^{2}$ por $R$ tenemos que 


$$
\begin{gathered}
A+\Omega^{2} \mu \widehat{B}-\Omega^{2} \mu^{2}= \\
\left(\begin{array}{cccccc}
f_{1} & -\frac{\mu(2 R-2)(4 R-3)}{4(1-R)^{\frac{3}{2}}} & \frac{6 R-3}{8(1-R)^{\frac{3}{2}}}-\frac{2 R-1}{8(1-R)^{\frac{3}{2}}} & 0 & 2 R-2 & 0 \\
-\frac{\mu(8 R-6)}{4(1-R)^{\frac{3}{2}}} & f_{2} & 0 & \frac{1}{8(1-R)^{\frac{3}{2}}} & 0 & 1 \\
\frac{6 R-3}{8(1-R)^{\frac{3}{2}}-\frac{2 R-1}{8(1-R)^{\frac{3}{2}}}} & 0 & f_{1} & -\frac{\mu(2 R-2)(4 R-3)}{4(1-R)^{\frac{3}{2}}} & 2 R-2 & 0 \\
0 & \frac{1}{8(1-R)^{\frac{3}{2}}} & -\frac{\mu(8 R-6)}{4(1-R)^{\frac{3}{2}}} & f_{2} & 0 & 1 \\
-2 & 0 & -2 & 0 & f_{3} & \frac{\mu(8 R-6)}{4(1-R)^{\frac{3}{2}}} \\
0 & 1 & 0 & 1 & -\frac{\mu(8 R-6)}{4(1-R)^{\frac{3}{2}}} & f_{4}
\end{array}\right)
\end{gathered}
$$

donde

$$
\begin{aligned}
& f_{1}=\frac{R-2}{\sqrt{1-R}}+\frac{4 R-1}{8(1-R)^{\frac{3}{2}}}+\frac{4 R-3}{4(1-R)^{\frac{3}{2}}}-\frac{6 R-3}{8(1-R)^{\frac{3}{2}}}-\frac{3 R(4 R-3)}{4(1-R)^{\frac{3}{2}}}-\frac{\mu^{2}(4 R-3)}{4(1-R)^{\frac{3}{2}}} \\
& f_{2}=\sqrt{1-R}+\frac{2 R-1}{8(1-R)^{\frac{3}{2}}}+\frac{4 R-3}{4(1-R)^{\frac{3}{2}}}-\frac{R(4 R-3)}{4(1-R)^{\frac{3}{2}}}-\frac{\mu^{2}(4 R-3)}{4(1-R)^{\frac{3}{2}}}, \\
& f_{3}=\frac{4 R-3}{4(1-R)^{\frac{3}{2}}}-4 \sqrt{1-R}-\frac{\mu^{2}(4 R-3)}{4(1-R)^{\frac{3}{2}}} \\
& f_{4}=2 \sqrt{1-R}+\frac{4 R-3}{4(1-R)^{\frac{3}{2}}}-\frac{\mu^{2}(4 R-3)}{4(1-R)^{\frac{3}{2}}} .
\end{aligned}
$$

De nuevo hacemos $M=\mu^{2}$ para obtener el polinomio $\widehat{p}(M)$, recordemos que necesitamos raíces reales negativas de $\widehat{p}$. Después de multiplicar al $\widehat{p}$ por el factor $-4096(1-R)^{9} /(4 R-3)^{3}$ obtenemos

$$
\widehat{p}(M)=M(M+1) Q_{1}(M) Q_{2}(M)
$$

donde

$$
\begin{aligned}
Q_{1}(M)= & 6 R+4 M R-3 M-3 \\
Q_{2}(M)= & 16 M^{3} R^{2}-24 M^{3} R+9 M^{3}+32 M^{2} R^{3}-64 M^{2} R^{2}+42 M^{2} R-9 M^{2}-256 M R^{4} \\
& +1008 M R^{3}-1512 M R^{2}+1020 M R-261 M+256 R^{5}-1280 R^{4}+2544 R^{3} \\
& -2520 R^{2}+1242 R-243 \\
= & \left(16 R^{2}-24 R+9\right) M^{3} \\
& +\left(32 R^{3}-64 R^{2}+42 R-9\right) M^{2} \\
& +\left(-256 R^{4}+1008 R^{3}-1512 R^{2}+1020 R-261\right) M \\
& +256 R^{5}-1280 R^{4}+2544 R^{3}-2520 R^{2}+1242 R-243 .
\end{aligned}
$$


El polinomio $Q_{1}(M)$ tiene raíz $M_{0}=\frac{3-6 R}{4 R-3}<0$ para $R \in\left(\frac{3}{4}, 1\right)$. Analizaremos las raíces del polinomio $Q_{2}(M)$, reescribiéndolo

$$
Q_{2}(M)=g_{1}(R) M^{3}+g_{2}(R) M^{2}+g^{3}(R) M+g_{4}(R) .
$$

Analicemos el polinomio $g_{4}(R)$, las raíces de su derivada, $g_{4}^{\prime}(R)$, son

$$
\begin{aligned}
& R_{1}=2.261009058898779+1.740181839315766 i, \\
& R_{2}=2.261009058898779-1.740181839315766 i, \\
& R_{3}=-0.261009058898779+0.198884034968046 i, \\
& R_{4}=-0.261009058898779-0.198884034968046 i,
\end{aligned}
$$

como son complejas el polinomio $g_{4}(R)$ no tiene máximos o mínimos locales, además $g_{4}\left(\frac{3}{4}\right)$, $g_{4}(1)<0$, concluyendo que $g_{4}(R)<0$ para $R \in\left(\frac{3}{4}, 1\right)$. El polinomio $g_{3}(R)$ tiene un máximo global en $R_{1}=0.920954761872277$, con $g_{3}\left(R_{1}\right)<0$, luego $g_{3}(R)<0$ para $R \in\left(\frac{3}{4}, 1\right)$. Tenemos también que $g_{1}(R)>0$, excepto para $R=\frac{3}{4}, g_{1}\left(\frac{3}{4}\right)=0$, , ver figuras (5.8), (5.9) y (5.10). Entonces independientemente del valor de $g_{2}(R)$ el polinomio $Q_{2}(M)$ tiene una variación de signo, lo que indica que existe una raíz real positiva. Esta raíz real positiva nos muestra la existencia de eigenvalores reales de la matriz $D f$. Luego podemos concluir que las órbitas que generan estos valores propios son inestables.

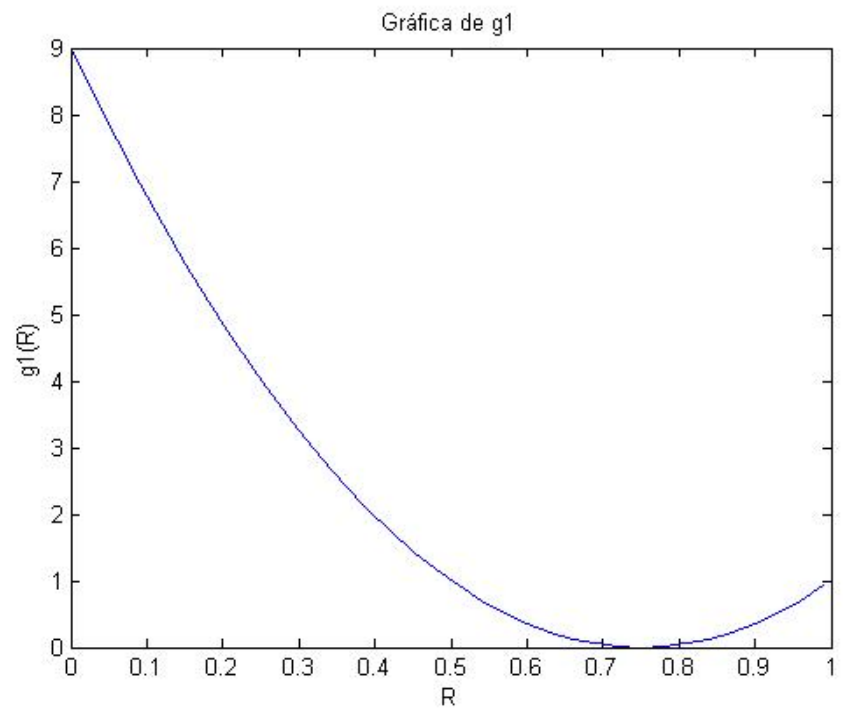

Figura 5.8: Gráfica de $g_{1}(R)$. 


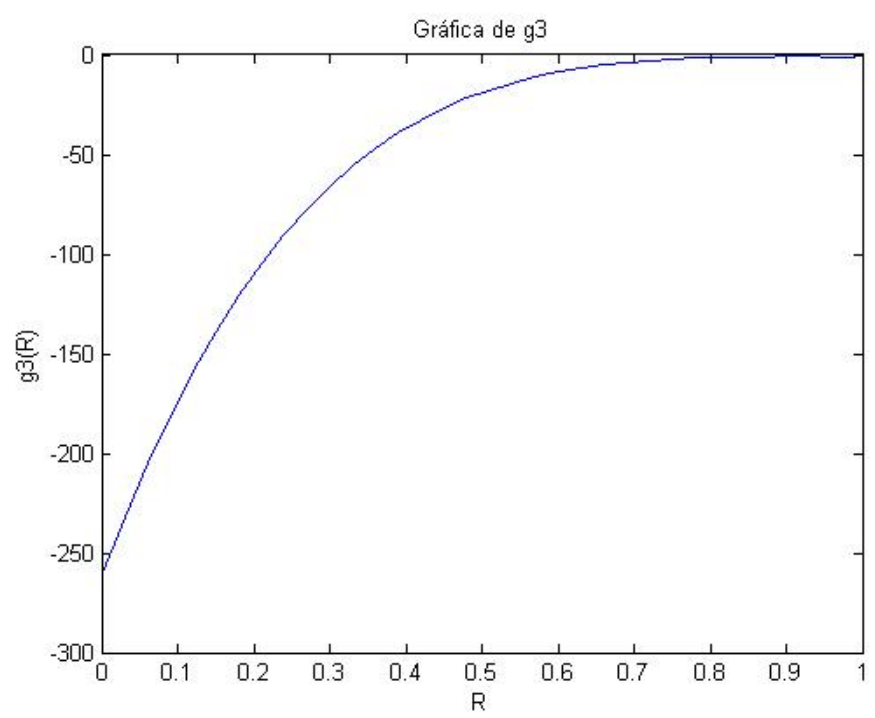

Figura 5.9: Gráfica de $g_{3}(R)$.

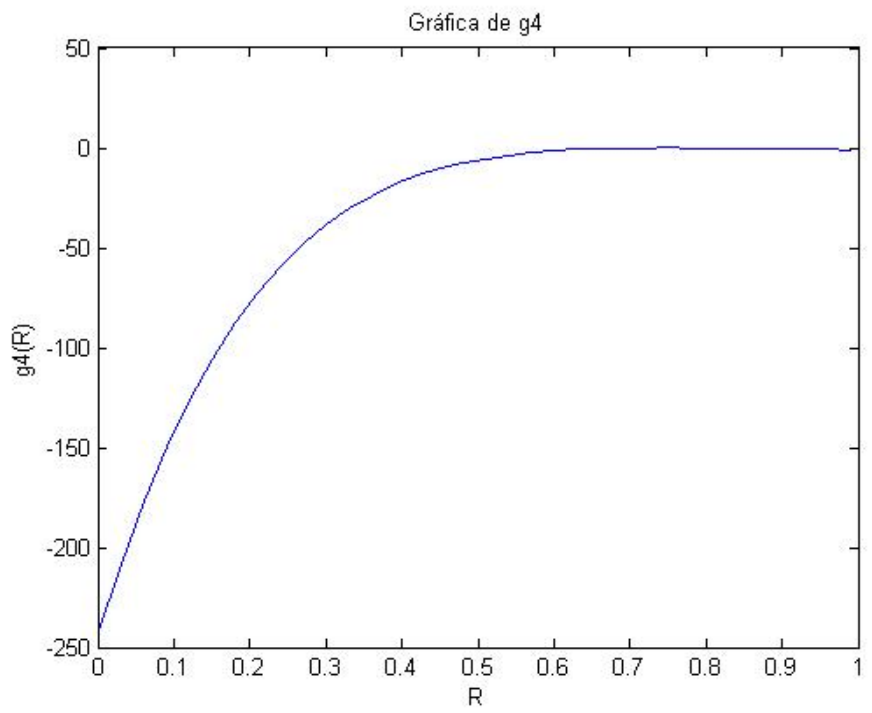

Figura 5.10: Gráfica de $g_{4}(R)$.

\subsection{3. $\quad 2+1$ Cuerpos}

Como en el problema newtoniano, el llamado problema $2+1$-cuerpos consiste en estudiar el problema de tres cuerpos, en donde la masa de dos de ellos es despreciable, y no existe 
fuerza de atracción sobre ellos. Hemos analizado los equilibrios relativos de este problema en $S^{2}$ obteniendo el siguiente resultado

Teorema 5.2.3 Consideremos órbitas eulerianas del problema curvado de tres cuerpos en $S^{2}$, con dos masas despreciables fijas en los lados opuestos de un círculo que rota uniformemente en un plano paralelo al ecuador $z=0, y$ una partícula con masa $m$ fija en el polo norte $(0,0,1)$, entonces para $z \in(0,1)$ las órbitas generadas son estables.

\section{Demostración}

En este caso tenemos $m_{1}=m_{2}=m$ con masas despreciables en los extremos de un círculo a altura $z$ y $m_{3}=1$ fija en el punto $(0,0,1)$. Como las masas $m_{1}$ y $m_{2}$ las suponemos muy pequeñas entonces no hay fuerza de atracción sobre ellas, cada una de éstas se mueve por la fuerza de atracción que ejerce la partícula $m_{3}$. Entonces tenemos dos sistemas desacoplados, uno formado por $m_{1}$ y $m_{3}$, y el otro sistema formado por $m_{2}$ y $m_{3}$.

Consideremos un sistema formado por las partículas $m_{1}$ y $m_{3}$ como anteriormente lo hicimos tomamos coordenadas rotatorias del sistema que gira a velocidad $\Omega$, aplicando la matriz de rotación a la posición de las partículas

$$
\left(\begin{array}{c}
X_{i} \\
Y_{i}
\end{array}\right)=R(\Omega \tau)\left(\begin{array}{c}
\xi_{i} \\
\eta_{i}
\end{array}\right)
$$

donde $R(\Omega \tau)$ es la matriz de rotación

$$
R(\Omega \tau)=\left(\begin{array}{cc}
\cos \Omega \tau & -\sin \Omega \tau \\
\sin \Omega \tau & \cos \Omega \tau
\end{array}\right) .
$$

De manera análoga obtenemos las ecuaciones de movimiento en el sistema rotatorio

$$
\begin{aligned}
\left(\begin{array}{c}
\xi_{i}^{\prime \prime} \\
\eta_{i}^{\prime \prime}
\end{array}\right) & =2 \Omega\left(\begin{array}{c}
\eta_{i}^{\prime} \\
-\xi_{i}^{\prime}
\end{array}\right)+\Omega^{2}\left(\begin{array}{c}
\xi_{i} \\
\eta_{i}
\end{array}\right)-r^{2} h_{i}\left(\begin{array}{c}
\xi_{i} \\
\eta_{i}
\end{array}\right) \\
& +m_{j}\left[\xi_{i}^{2}+\eta_{i}^{2}+\xi_{j}^{2}+\eta_{j}^{2}-2\left(\xi_{i} \xi_{j}+\eta_{i} \eta_{j}\right) \sqrt{\left(1-r^{2}\left(\xi_{i}^{2}+\eta_{i}^{2}\right)\right)\left(1-r^{2}\left(\xi_{j}^{2}+\eta_{j}^{2}\right)\right)}\right. \\
& \left.-r^{2}\left(\left(\xi_{i} \xi_{j}+\eta_{i} \eta_{j}\right)^{2}+\left(\xi_{i}^{2}+\eta_{i}^{2}\right)\left(\xi_{j}^{2}+\eta_{j}^{2}\right)\right)\right]^{-3 / 2}\left[\left(\begin{array}{c}
\xi_{j} \\
\eta_{j}
\end{array}\right)-\left(r^{2}\left(\xi_{i} \xi_{j}+\eta_{i} \eta_{j}\right)\right.\right. \\
& \left.\left.+\sqrt{\left(1-r^{2}\left(\xi_{i}^{2}+\eta_{i}^{2}\right)\right)\left(1-r^{2}\left(\xi_{j}^{2}+\eta_{j}^{2}\right)\right)}\right)\left(\begin{array}{c}
\xi_{i} \\
\eta_{i}
\end{array}\right)\right],
\end{aligned}
$$

donde 


$$
h_{i}=\Omega^{2}\left(\xi_{i}^{2}+\eta_{i}^{2}\right)+2 \Omega\left(\xi_{i} \eta_{i}^{\prime}-\eta_{i} \xi_{i}^{\prime}\right)+\left(\left(\xi_{i}^{\prime 2}\right)+\left(\eta_{i}^{\prime 2}\right)\right)+\frac{r^{2}}{1-r^{2}\left(\xi_{i}^{2}+\eta_{i}^{2}\right)}\left(\xi_{i} \xi_{i}^{\prime}+\eta_{i} \eta_{i}^{\prime}\right)^{2}
$$

para $i=1,2$.

En el sistema podemos ver que un punto fijo es

$$
\xi_{1}=1, \eta_{1}=0, \xi_{2}=0, \eta_{2}=0 \xi_{1}^{\prime}=0, \eta_{1}^{\prime},=0, \xi_{2}^{\prime}=0, \eta_{2}^{\prime}=0
$$

La matriz $D f$ correspondiente es una matriz de tamaño $8 \times 8$ de la forma

$$
D f=\left(\begin{array}{cc}
0 & I \\
A & B
\end{array}\right)
$$

en donde $I$ es la matriz identidad de tamaño $4 \times 4$. La matriz $A$ en este caso tiene la forma

$$
A=\left(\begin{array}{llll}
a & 0 & b & 0 \\
0 & c & 0 & d \\
b & 0 & a & 0 \\
0 & d & 0 & c
\end{array}\right)
$$

donde

$$
\begin{aligned}
a & =\frac{r^{2}}{\sqrt{1-r^{2}}}+\Omega^{2}-3 \Omega^{2} r^{2}+2 \sqrt{1-r^{2}}, \\
b & =2 r^{2}-2 \\
c & =\Omega^{2}-\Omega^{2} r^{2}-\sqrt{1-r^{2}}, \\
d & =1 .
\end{aligned}
$$

La matriz $B$

$$
B=\left(\begin{array}{rrrr}
0 & 2 \Omega-2 \Omega r^{2} & 0 & 0 \\
-2 \Omega & 0 & 0 & 0 \\
0 & 0 & 0 & 2 \Omega \\
0 & 0 & -2 \Omega & 0
\end{array}\right)
$$

En este problema la velocidad angular debe cumplir

$$
\Omega^{2}=\frac{4\left(1-r^{2}\right)+m}{4\left(1-r^{2}\right)^{3 / 2}}
$$

Los vectores propios de $D f$ son los ceros de la ecuación $\operatorname{det}(D f-\lambda I)=0$. Definamos $\mu$ tal que $\lambda=\Omega \mu$, por lo que buscaremos los ceros de la ecuación $\operatorname{det}(D f-\Omega \mu I)=0$. La condicón que buscaremos para los valores propios puede ser escrita como 


$$
\left(\begin{array}{cc}
0 & I \\
A & \Omega \widehat{B}
\end{array}\right)\left(\begin{array}{l}
u \\
v
\end{array}\right)=\Omega \mu\left(\begin{array}{l}
u \\
v
\end{array}\right),
$$

donde $u, v$ están formados por las primeras y últimas cuatro componentes, respectivamente, del eigenvector correspondiente al eigenvalor $\Omega \mu$.

Así la condición del eigenvalor con parámetro $\mu$ se sigue de

$$
v=\Omega \mu u, \quad A u+\Omega \widehat{B} \Omega \mu u=\Omega \mu v=\Omega^{2} \mu^{2} u .
$$

Denotemos al polinomio caracteristico como $p(\mu)$. La condición de valor propio debe satisfacer

$$
p(\mu)=\operatorname{det}\left(A+\Omega^{2} \mu \widehat{B}-\Omega^{2} \mu^{2} I\right)=0 .
$$

Si denotamos a $r^{2}$ por $R$ entonces

$$
A+\Omega^{2} \mu \widehat{B}-\Omega^{2} \mu^{2} I=
$$

$$
\left(\begin{array}{cccc}
f_{1} & \mu\left(\frac{m-4 R+4}{2(1-R)^{\frac{3}{2}}}-\frac{R(m-4 R+4)}{2(1-R)^{\frac{3}{2}}}\right) & 2 R-2 & 0 \\
-\frac{\mu(m-4 R+4)}{2(1-R)^{\frac{3}{2}}} & f_{2} & 0 & 1 \\
-2 m & 0 & f_{3} & \frac{\mu(m-4 R+4)}{2(1-R)^{\frac{3}{2}}} \\
0 & m & -\frac{m(m-4 R+4)}{2(1-R)^{\frac{3}{2}}} & f_{4}
\end{array}\right),
$$

donde

$$
\begin{aligned}
& f_{1}=\frac{R}{\sqrt{1-R}}+\frac{m-4 R+4}{4(1-R)^{\frac{3}{2}}}+2 \sqrt{1-R}-\frac{3 R(m-4 R+4)}{4(1-R)^{\frac{3}{2}}}-\frac{\mu^{2}(m-4 R+4)}{4(1-R)^{\frac{3}{2}}}, \\
& f_{2}=\frac{m-4 R+4}{4(1-R)^{\frac{3}{2}}}-\sqrt{1-R}-\frac{R(m-4 R+4)}{4(1-R)^{\frac{3}{2}}}-\frac{\mu^{2}(m-4 R+4)}{4(1-R)^{\frac{3}{2}}} \\
& f_{3}=2 m \sqrt{1-R}+\frac{m-4 R+4}{4(1-R)^{\frac{3}{2}}}-\frac{\mu^{2}(m-4 R+4)}{4(1-R)^{\frac{3}{2}}} \\
& f_{4}=\frac{m-4 R+4}{4(1-R)^{\frac{3}{2}}}-m \sqrt{1-R}-\frac{\mu^{2}(m-4 R+4)}{4(1-R)^{\frac{3}{2}}} .
\end{aligned}
$$

Cuyo determinante es 


$$
\begin{aligned}
& p(\mu)=-\frac{1}{256(R-1)^{6}}\left(96 m^{4} R^{6}-512 m^{4} R^{5}+32 m^{4} R^{4} \mu^{4}+76 m^{4} R^{4} \mu^{2}+1108 m^{4} R^{4}\right. \\
& -128 m^{4} R^{3} \mu^{4}-296 m^{4} R^{3} \mu^{2}-1240 m^{4} R^{3}+4 m^{4} R^{2} \mu^{6}+193 m^{4} R^{2} \mu^{4} \\
& +749 m^{4} R^{2}-8 m^{4} R \mu^{6}-132 m^{4} R \mu^{4}-256 m^{4} R \mu^{2}-228 m^{4} R-m^{4} \mu^{8}+30 m^{4} \mu^{4} \\
& +56 m^{4} \mu^{2}+27 m^{4}-256 m^{3} R^{7}+256 m^{3} R^{6} \mu^{2}+2048 m^{3} R^{6}-256 m^{3} R^{5} \mu^{4} \\
& -1840 m^{3} R^{5} \mu^{2}-6608 m^{3} R^{5}+1344 m^{3} R^{4} \mu^{4}+5456 m^{3} R^{4} \mu^{2}+11376 m^{3} R^{4} \\
& -48 m^{3} R^{3} \mu^{6}-2824 m^{3} R^{3} \mu^{4}-8400 m^{3} R^{3} \mu^{2}-11352 m^{3} R^{3}+144 m^{3} R^{2} \mu^{6} \\
& +2988 m^{3} R^{2} \mu^{4}+7064 m^{3} R^{2} \mu^{2}+6556 m^{3} R^{2}+16 m^{3} R \mu^{8}-84 m^{3} R \mu^{6} \\
& -1528 m^{3} R \mu^{4}-3060 m^{3} R \mu^{2}-2016 m^{3} R-16 m^{3} \mu^{8}-12 m^{3} \mu^{6}+276 m^{3} \mu^{4} \\
& +524 m^{3} \mu^{2}+252 m^{3}-1024 m^{2} R^{7} \mu^{2}-1024 m^{2} R^{7}+512 m^{2} R^{6} \mu^{4}+7168 m^{2} R^{6} \mu^{2} \\
& +6656 m^{2} R^{6}-3584 m^{2} R^{5} \mu^{4}-22528 m^{2} R^{5} \mu^{2}-18944 m^{2} R^{5}+192 m^{2} R^{4} \mu^{6} \\
& +10256 m^{2} R^{4} \mu^{4}+40416 m^{2} R^{4} \mu^{2}+30352 m^{2} R^{4}-768 m^{2} R^{3} \mu^{6}-15488 m^{2} R^{3} \mu^{4} \\
& -44032 m^{2} R^{3} \mu^{2}-29312 m^{2} R^{3}-96 m^{2} R^{2} \mu^{8}+816 m^{2} R^{2} \mu^{6}+12704 m^{2} R^{2} \mu^{4} \\
& +28720 m^{2} R^{2} \mu^{2}+16928 m^{2} R^{2}+192 m^{2} R \mu^{8}-96 m^{2} R \mu^{6}-5120 m^{2} R \mu^{4} \\
& -10208 m^{2} R \mu^{2}-5376 m^{2} R-96 m^{2} \mu^{8}-144 m^{2} \mu^{6}+720 m^{2} \mu^{4}+1488 m^{2} \mu^{2} \\
& +1024 m R^{6} \mu^{4}+2048 m R^{6} \mu^{2}+1024 m R^{6}-256 m R^{5} \mu^{6}-6144 m R^{5} \mu^{4} \\
& -11520 m R^{5} \mu^{2}-5632 m R^{5}+1280 m R^{4} \mu^{6}+15424 m R^{4} \mu^{4}+27008 m R^{4} \mu^{2} \\
& +12864 m R^{4}+256 m R^{3} \mu^{8}-1728 m R^{3} \mu^{6}-19840 m R^{3} \mu^{4}-33472 m R^{3} \mu^{2} \\
& -15616 m R^{3}-768 m R^{2} \mu^{8}+64 m R^{2} \mu^{6}+13056 m R^{2} \mu^{4}+22848 m R^{2} \mu^{2} \\
& +10624 m R^{2}+768 m R \mu^{8}+1216 m R \mu^{6}-3712 m R \mu^{4}-8000 m R \mu^{2}-3840 m R \\
& -256 m \mu^{8}-576 m \mu^{6}+192 m \mu^{4}+1088 m \mu^{2}+576 m-256 R^{4} \mu^{8}-768 R^{4} \mu^{6} \\
& -768 R^{4} \mu^{4}-256 R^{4} \mu^{2}+1024 R^{3} \mu^{8}+3072 R^{3} \mu^{6}+3072 R^{3} \mu^{4}+1024 R^{3} \mu^{2} \\
& -1536 R^{2} \mu^{8}-4608 R^{2} \mu^{6}-4608 R^{2} \mu^{4}-1536 R^{2} \mu^{2}+1024 R \mu^{8}+3072 R \mu^{6} \\
& \left.+3072 R \mu^{4}+1024 R \mu^{2}-256 \mu^{8}-768 \mu^{6}-768 \mu^{4}-256 \mu^{2}+418 m^{4} R^{2} \mu^{2}+720 m^{2}\right) \text {. }
\end{aligned}
$$

Suponemos que la masa $m$ es despreciable, entonces tomando $\lim _{m \rightarrow 0} p(\mu)$, obtenemos

$$
p(\mu)=\frac{\mu^{2}\left(\mu^{2}+1\right)^{3}}{(R-1)^{2}}
$$

mostrando que las órbitas generadas son estables, ya que los valores propios de la matriz $D f$ dados por las raíces de $p(\mu)$ son iguales a cero y puramente imaginarias.

Con este resultado concluimos nuestro trabajo referente a los equilibrios relativos en $S^{2}$, mostrando resultados originales sobre equilibrios relativos eulerianos del problema de dos 
y tres cuerpos. Cabe mencionar que en [25], los autores Regina Martínez y Carles Simó, estudian movimientos lagrangianos de tres cuerpos con masas iguales en $S^{2}$, entre ellos se analizan equilibrios relativos Lagrangianos, mostrando rangos de valores de momento angular en donde las órbitas son espectralmente estables. También es importante señalar que en su trabajo se utilizan algunos argumentos numéricos.

Como mencionamos en la introducción, con este trabajo en el que estamos mostrando resultados originales y con [25] se tiene un claro panorama sobre los movimientos que generan los equlibrios relativos. En [25] se estudian los equilibrios relativos del tipo lagrangiano y nosotros analizamos los equilibrios relativos del tipo euleriano del problema de tres cuerpos.

Es importante destacar que nosotros también estudiamos la estabilidad del problema de dos cuerpos, señalando que todos los resultados expuestos en este capítulo no se habían realizado antes.

Ya que analizamos el problema en espacios de curvatura constante positiva veremos qué es lo que pasa si proponemos el problema en espacios de curvatura negativa, los resultados los exponemos en el siguiente capítulo. 
Capítulo 6

\section{Estabilidad de Equilibrios Relativos en $H^{2}$}

Es esta sección mostraremos un análisis de la estabilidad espectral de los equilibrios relativos eulerianos en $H^{2}$, mostraremos la condición de estabilidad en el problema de equilibrios relativos elípticos e hiperbólicos del problema de dos y tres cuerpos. Recordemos que no existen equilibrios relativos del tipo parabólico en $H^{2}$.

\subsection{Equilibrios Relativos Elípticos}

Comenzaremos analizando los equilibrios relativos elipticos en $H^{2}$, recordemos que éstos son soluciones de las ecuaciones de movimiento en donde las partículas se encuentran girando en planos paralelos al plano $x y$, esta definición la expusimos en la sección (4.1). Mostraremos resultados sobre el problema de dos y tres cuerpos.

\subsubsection{Problema de Dos Cuerpos}

Comenzamos con el caso más sencillo que es el problema de dos cuerpos. Tenemos el siguiente resultado en donde tenemos dos masas iguales girando a una altura $z$, ver figura (6.1). 


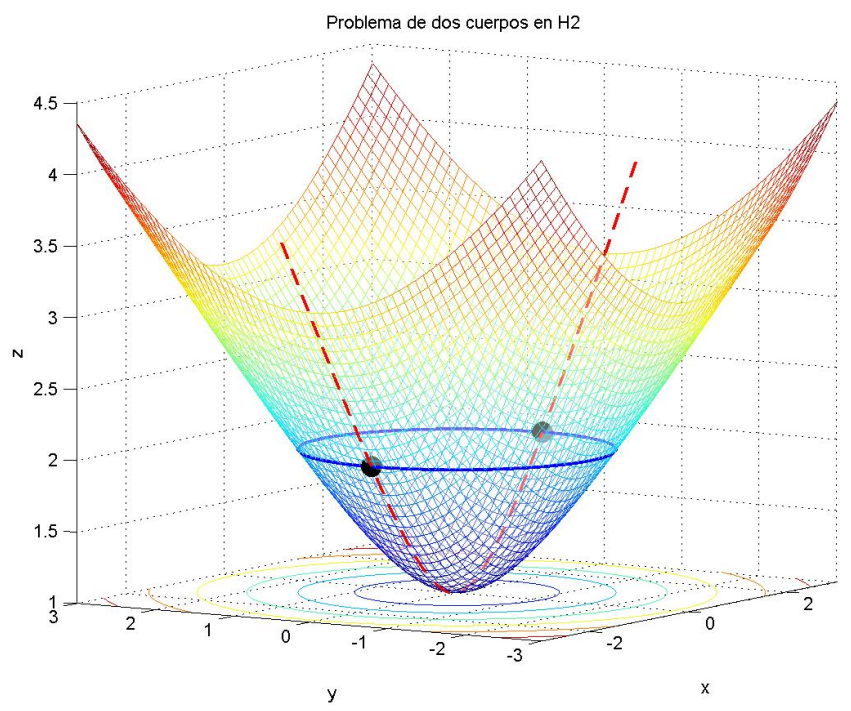

Figura 6.1: Equilibrios relativos elípticos del problema de dos cuerpos en $H^{2}$. Dos cuerpos se encuentran girando sobre órbitas circulares a una altura $z$ (curva continua-azul), manteniéndose siempre la misma distancia, medida sobre geodésicas (curva punteada-roja).

Teorema 6.1.1 Consideremos órbitas periódicas del problema curvado de dos cuerpos en $H^{2}$. Si los cuerpos con masas $m_{1}=m_{2}$ se encuentran fijos en los lados opuestos de un círulo que gira uniformemente en un plano paralelo al plano $x y$, entonces para $z \in(1, \infty)$ las órbitas generadas son estables si $1<z<\frac{\sqrt{3}}{\sqrt{2}}$ e inestables si $z>\frac{\sqrt{3}}{\sqrt{2}}$.

\section{Demostración}

Al normalizar las masas tenemos $m_{1}=m_{2}=1$. Como en $S^{2}$, introducimos coordenadas rotatorias, aplicando a las coordenadas de posición de los cuerpos la correspondiente matriz de rotación. Es decir, introducimos coordenadas $\left(\xi_{i}, \eta_{i}\right), i=1,2$, tales que

$$
\left(\begin{array}{c}
X_{i} \\
Y_{i}
\end{array}\right)=R(\Omega \tau)\left(\begin{array}{c}
\xi_{i} \\
\eta_{i}
\end{array}\right)
$$

en donde $R(\Omega \tau)$ es la matriz de rotación

$$
R(\Omega \tau)=\left(\begin{array}{cc}
\cos \Omega \tau & -\sin \Omega \tau \\
\sin \Omega \tau & \cos \Omega \tau
\end{array}\right)
$$

Reescribiendo las ecuaciones de movimiento en términos de $\left(\xi_{i}, \eta_{i}\right)$ obtenemos las ecuaciones de movimiento en $H^{2}$ en un sistema rotatorio que gira a velocidad $\Omega$ 


$$
\begin{aligned}
\left(\begin{array}{c}
\xi_{i}^{\prime \prime} \\
\eta_{i}^{\prime \prime}
\end{array}\right) & =2 \Omega\left(\begin{array}{c}
\eta_{i}^{\prime} \\
-\xi_{i}^{\prime}
\end{array}\right)+\Omega^{2}\left(\begin{array}{c}
\xi_{i} \\
\eta_{i}
\end{array}\right)+r^{2} h_{i}\left(\begin{array}{c}
\xi_{i} \\
\eta_{i}
\end{array}\right) \\
& +m_{j}\left[\xi_{i}^{2}+\eta_{i}^{2}+\xi_{j}^{2}+\eta_{j}^{2}-2\left(\xi_{i} \xi_{j}+\eta_{i} \eta_{j}\right) \sqrt{\left(1+r^{2}\left(\xi_{i}^{2}+\eta_{i}^{2}\right)\right)\left(1+r^{2}\left(\xi_{j}^{2}+\eta_{j}^{2}\right)\right)}\right. \\
& \left.+r^{2}\left(\left(\xi_{i} \xi_{j}+\eta_{i} \eta_{j}\right)^{2}+\left(\xi_{i}^{2}+\eta_{i}^{2}\right)\left(\xi_{j}^{2}+\eta_{j}^{2}\right)\right)\right]^{-3 / 2}\left[\left(\begin{array}{c}
\xi_{j} \\
\eta_{j}
\end{array}\right)+\left(r^{2}\left(\xi_{i} \xi_{j}+\eta_{i} \eta_{j}\right)\right.\right. \\
& \left.\left.-\sqrt{\left(1+r^{2}\left(\xi_{i}^{2}+\eta_{i}^{2}\right)\right)\left(1+r^{2}\left(\xi_{j}^{2}+\eta_{j}^{2}\right)\right)}\right)\left(\begin{array}{c}
\xi_{i} \\
\eta_{i}
\end{array}\right)\right]
\end{aligned}
$$

donde

$$
h_{i}=\Omega^{2}\left(\xi_{i}^{2}+\eta_{i}^{2}\right)+2 \Omega\left(\xi_{i} \eta_{i}^{\prime}-\eta_{i} \xi_{i}^{\prime}\right)+\left(\left(\xi_{i}^{\prime 2}\right)+\left(\eta_{i}^{\prime 2}\right)\right)-\frac{r^{2}}{1+r^{2}\left(\xi_{i}^{2}+\eta_{i}^{2}\right)}\left(\xi_{i} \xi_{i}^{\prime}+\eta_{i} \eta_{i}^{\prime}\right)^{2}
$$

para $i=1,2$.

En (6.3) comprobamos que

$$
\xi_{1}=1, \eta_{1}=0, \xi_{2}=-1, \eta_{2}=0, \xi_{1}^{\prime}=0, \eta_{1}^{\prime}=0, \xi_{2}^{\prime}=0, \eta_{2}^{\prime}=0
$$

es punto fijo del sistema.

La correspondiente matriz $D f$ correspondiente es una matriz de tamaño $8 \times 8$ de la forma

$$
D f=\left(\begin{array}{ll}
0 & I \\
A & B
\end{array}\right)
$$

en donde $I$ es la matriz identidad de tamaño $4 \times 4$. La matriz $A$ en este caso tiene la forma

$$
A=\left(\begin{array}{llll}
a & 0 & b & 0 \\
0 & c & 0 & d \\
b & 0 & a & 0 \\
0 & d & 0 & c
\end{array}\right)
$$

donde 


$$
\begin{aligned}
& a=\Omega^{2}+3 \Omega^{2} r^{2}-\frac{1+4 r^{2}}{\left(4+4 r^{2}\right)^{3 / 2}}+\frac{\left(6+6 r^{2}\right)\left(4+8 r^{2}\right)}{2\left(4+4 r^{2}\right)^{5 / 2}}, \\
& b=\frac{1+2 r^{2}}{\left(4+4 r^{2}\right)^{3 / 2}}-\frac{\left(1+6 r^{2}\right)\left(4+8 r^{2}\right)}{2\left(4+4 r^{2}\right)^{5 / 2}}, \\
& c=-\frac{2 r^{2}+1}{\left(4+4 r^{2}\right)^{3 / 2}}+\Omega^{2}+\Omega^{2} r^{2}, \\
& d=\frac{1}{\left(4+4 r^{2}\right)^{3 / 2}} .
\end{aligned}
$$

La matriz $B$

$$
B=\left(\begin{array}{rrrr}
0 & 2 \Omega+2 \Omega r^{2} & 0 & 0 \\
-2 \Omega & 0 & 0 & 0 \\
0 & 0 & 0 & 2 \Omega+2 \Omega r^{2} \\
0 & 0 & -2 \Omega & 0
\end{array}\right) .
$$

En el problema restringido la velodidad angular debe cumplir

$$
\Omega^{2}=\frac{1}{4\left(1+r^{2}\right)^{3 / 2}} .
$$

Usando (6.9) podemos reescribir a la matriz $D f$ como

$$
D f=\left(\begin{array}{cc}
0 & I \\
\Omega^{2} \widehat{A} & \Omega \widehat{B}
\end{array}\right) .
$$

Los eigenvalores de la matriz $D f$ son los ceros de la ecuación $\operatorname{det}(D f-\lambda I)=0$. Definamos $\mu$ de tal manera que $\lambda=\Omega \mu$, entonces buscaremos los ceros de la ecuación $\operatorname{det}(D f-\Omega \mu I)=0$.

Analogamente a la sección 5.2.1 podemos ver que la condición debe satisfacer $D(\mu) u=0$, donde $D(\mu)=\widehat{A}+\mu \widehat{B}-\mu^{2} I$, y se debe tener $D=0$.

Denotamos a $r^{2}$ por $R$. El polinomio característico $p(\mu)$ de la matriz $\widehat{A}+\mu \widehat{B}-\mu^{2} I$ es

$$
p(\mu)=\mu^{8}+3 \mu^{6}-4 \mu^{4} R^{2}+3 \mu^{4}-4 \mu^{2} R^{2}+\mu^{2} .
$$

El polinomio $p(\mu)$ lo podemos escribir como

$$
p(\mu)=\mu^{2}\left(\mu^{2}+1\right)\left(\mu^{2}-2 R+1\right)\left(\mu^{2}+2 R+1\right) .
$$


En la sección 5.2.1 hicimos el análisis del mismo polinomio y vimos que $D f$ tiene eigenvalores puramente complejos si

$$
2 R-1 \leq 0
$$

con $R \in(0,1)$.

La condición (6.13) se cumple cuando $R \in\left(0, \frac{1}{2}\right]$, es decir, cuando $z^{2} \in\left[1, \frac{3}{2}\right)$.

\subsubsection{Problema de Tres Cuerpos}

A continuación mostraremos nuestros resultados acerca de equilibrios relativos eulerianos, el caso restringido, el caso de masas iguales y el problema $2+1$ cuerpos, ver figura (6.2).

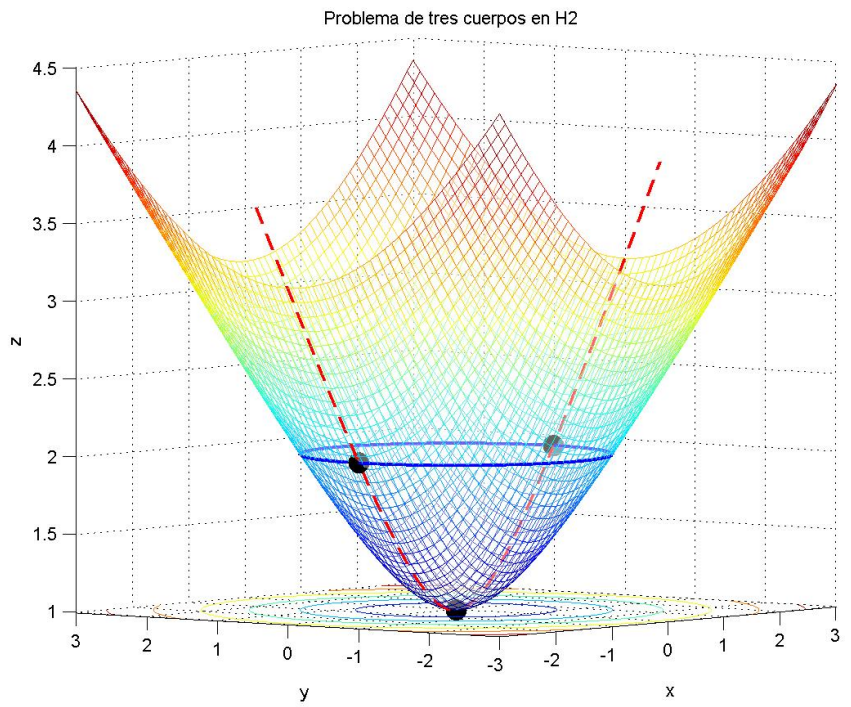

Figura 6.2: Equilibrios relativos elípticos del problema de tres cuerpos en $H^{2}$. Un cuerpo se encuentra fijo en el punto $(0,0,1)$ y los otros dos se encuentran girando a una altura $z$ formando órbitas circulares (curva continua-azul), los cuerpos se encuentran manteniendo siempre la misma distancia medida sobre geodésicas (curva punteada-roja).

\subsubsection{Problema Restringido}

Lo que obtuvimos acerca del problema restringido lo mostramos en el siguiente

Teorema 6.1.2 Consideremos órbitas eulerianas del problema curvado de tres cuerpos en $H^{2}$, con masas $m_{1}=m_{2}$ fijas en los lados opuestos de un círculo que gira uniformemete un 
un plano paralelo al plano xy a altura $z$, y la partícula $m_{3}$ con masa despreciable fija en el punto $(0,0,1)$, entonces para $z \in(1, \infty)$ las órbitas generadas son inestables.

\section{Demostración}

Para demostrar este resultado introduciremos una partícula de masa $m_{3}=m$ fija en el punto $(0,0,1)$, y después haremos $m \rightarrow 0$, además normalizaremos el valor de las otras dos masas, es decir tomaremos $m_{1}=m_{2}=1$. Introduciremos coordenadas rotatorias para estudiar la estabilidad de equilibrios relativos, consideremos los siguientes cambios de coordenadas

$$
\begin{aligned}
x_{i}=r X_{i}, \quad y_{i} & =r Y_{i}, \quad r \in(0, \infty), \\
t & =r^{\frac{3}{2}} \tau,
\end{aligned}
$$

tendremos entonces las ecuaciones de movimiento en términos de

$$
Q=(X, Y, z) \text {. }
$$

Hacemos tambien un reescalamiento sobre el valor de la velocidad angular, $\Omega=\frac{\omega t}{\tau}$. De esta manera tenemos

$$
\begin{aligned}
\Omega^{2} & =\frac{\omega^{2} t^{2}}{\tau^{2}} \\
& =\frac{\omega^{2} r^{3} \tau^{2}}{\tau^{2}} \\
& =\omega^{2} r^{3} \\
& =\frac{4 m_{3} z^{2}+m_{1}}{4 z^{3}\left(z^{2}-1\right)} r^{3} \\
& =\frac{m_{1}+4 m_{3}\left(1+r^{2}\right)}{4\left(1+r^{2}\right)^{\frac{3}{2}}} \\
& =\frac{1+4 m\left(1+r^{2}\right)}{4\left(1+r^{2}\right)^{\frac{3}{2}}} .
\end{aligned}
$$

De forma análoga que en $S^{2}$ tenemos

$$
\begin{aligned}
X_{i}^{\prime} & =r^{\frac{1}{2}} \dot{x}_{i}, \\
X_{i}^{\prime \prime} & =r^{2} \ddot{x}_{i}, \\
Y_{i}^{\prime} & =r^{\frac{1}{2}} \dot{y}_{i}, \\
Y_{i}^{\prime \prime} & =r^{2} \ddot{y}_{i},
\end{aligned}
$$


para $i=1,2,3$.

Ahora aplicamos las coordenadas reescaladas a las ecuaciones de movimiento, teniendo

$$
\begin{aligned}
\ddot{q}_{i} & =\sum_{j=1, j \neq i}^{3} \frac{m_{j}\left(q_{j}+\left(q_{i} \odot q_{j}\right) q_{i}\right)}{\left(\left(q_{i} \odot q_{j}\right)^{2}-1\right)^{\frac{3}{2}}}+\left(\dot{q}_{i} \odot \dot{q}_{i}\right) q_{i} \\
& =\sum_{j=1, j \neq i}^{3} \frac{m_{j}\left(\left(x_{j}, y_{j}, z_{j}\right)+\left(\left(x_{i}, y_{i}, z_{i}\right) \odot\left(x_{j}, y_{j}, z_{j}\right)\right)\left(x_{i}, y_{i}, z_{i}\right)\right)}{\left(\left(\left(x_{i}, y_{i}, z_{i}\right) \odot\left(x_{j}, y_{j}, z_{j}\right)\right)^{2}-1\right)^{\frac{3}{2}}} \\
& +\left(\left(\dot{x}_{i}, \dot{y}_{i}, \dot{z}_{i}\right) \odot\left(\dot{x}_{i}, \dot{y}_{i}, \dot{z}_{i}\right)\right)\left(x_{i}, y_{i}, z_{i}\right) \\
& =\sum_{j=1, j \neq i}^{3} \frac{m_{j}\left(\left(r X_{j}, r Y_{j}, z_{j}\right)+\left(\left(x_{i}, y_{i}, z_{i}\right) \odot\left(x_{j}, y_{j}, z_{j}\right)\right)\left(r X_{i}, r Y_{i}, z_{i}\right)\right)}{\left(\left(\left(x_{i}, y_{i}, z_{i}\right) \odot\left(x_{i}, y_{i}, z_{i}\right)\right)^{2}-1\right)^{\frac{3}{2}}} \\
& +\left(\left(\dot{x}_{i}, \dot{y}_{i}, \dot{z}_{i}\right) \odot\left(\dot{x}_{i}, \dot{y}_{i}, \dot{z}_{i}\right)\right)\left(r X_{i}, r Y_{i}, z_{i}\right),
\end{aligned}
$$

para $i=1,2,3$.

En los equilibrios relativos elipticos las órbitas giran de forma paralela al plano $z=0$, entonces las coorrespondientes coordenadas $z_{i}$ de las partículas se mantienen constantes, por lo que la omitiremos en el análisis.

Introducimos coordenadas rotatorias $\left(\xi_{i}, \eta_{i}\right), i=1,2,3$,

$$
\left(\begin{array}{c}
X_{i} \\
Y_{i}
\end{array}\right)=R(\Omega \tau)\left(\begin{array}{c}
\xi_{i} \\
\eta_{i}
\end{array}\right)
$$

en donde $R(\Omega \tau)$ es la matriz de rotación

$$
R(\Omega \tau)=\left(\begin{array}{cc}
\cos \Omega \tau & -\sin \Omega \tau \\
\sin \Omega \tau & \cos \Omega \tau
\end{array}\right)
$$

Reescribiendo las ecuaciones de movimiento en términos de $\left(\xi_{i}, \eta_{i}\right)$ como lo hicimos en el caso de $S^{2}$ obtenemos las ecuaciones de movimiento en $H^{2}$ en un sistema rotatorio que gira a velocidad $\Omega$ 


$$
\begin{aligned}
\left(\begin{array}{c}
\xi_{i}^{\prime \prime} \\
\eta_{i}^{\prime \prime}
\end{array}\right) & =2 \Omega\left(\begin{array}{c}
\eta_{i}^{\prime} \\
-\xi_{i}^{\prime}
\end{array}\right)+\Omega^{2}\left(\begin{array}{c}
\xi_{i} \\
\eta_{i}
\end{array}\right)+r^{2} h_{i}\left(\begin{array}{c}
\xi_{i} \\
\eta_{i}
\end{array}\right) \\
& +\sum_{j=1, j \neq i}^{3} m_{j}\left[\xi_{i}^{2}+\eta_{i}^{2}+\xi_{j}^{2}+\eta_{j}^{2}-2\left(\xi_{i} \xi_{j}+\eta_{i} \eta_{j}\right) \sqrt{\left(1+r^{2}\left(\xi_{i}^{2}+\eta_{i}^{2}\right)\right)\left(1+r^{2}\left(\xi_{j}^{2}+\eta_{j}^{2}\right)\right)}\right. \\
& \left.+r^{2}\left(\left(\xi_{i} \xi_{j}+\eta_{i} \eta_{j}\right)^{2}+\left(\xi_{i}^{2}+\eta_{i}^{2}\right)\left(\xi_{j}^{2}+\eta_{j}^{2}\right)\right)\right]^{-3 / 2}\left[\left(\begin{array}{c}
\xi_{j} \\
\eta_{j}
\end{array}\right)+\left(r^{2}\left(\xi_{i} \xi_{j}+\eta_{i} \eta_{j}\right)\right.\right. \\
& \left.\left.-\sqrt{\left(1+r^{2}\left(\xi_{i}^{2}+\eta_{i}^{2}\right)\right)\left(1+r^{2}\left(\xi_{j}^{2}+\eta_{j}^{2}\right)\right)}\right)\left(\begin{array}{c}
\xi_{i} \\
\eta_{i}
\end{array}\right)\right]
\end{aligned}
$$

donde

$$
h_{i}=\Omega^{2}\left(\xi_{i}^{2}+\eta_{i}^{2}\right)+2 \Omega\left(\xi_{i} \eta_{i}^{\prime}-\eta_{i} \xi_{i}^{\prime}\right)+\left(\left(\xi_{i}^{\prime 2}\right)+\left(\eta_{i}^{\prime 2}\right)\right)-\frac{r^{2}}{1+r^{2}\left(\xi_{i}^{2}+\eta_{i}^{2}\right)}\left(\xi_{i} \xi_{i}^{\prime}+\eta_{i} \eta_{i}^{\prime}\right)^{2},
$$

para $i=1,2,3$.

En (6.22) comprobamos que

$\xi_{1}=1, \eta_{1}=0, \xi_{2}=-1, \eta_{2}=0, \xi_{3}=0, \eta_{3}=0, \xi_{1}^{\prime}=0, \eta_{1}^{\prime},=0, \xi_{2}^{\prime}=0, \eta_{2}^{\prime}=0, \xi_{3}^{\prime}=0, \eta_{3}^{\prime}=0$

es punto fijo del sistema.

Estudiaremos los eigenvalores del campo vectorial de la diferencial de la función de la parte derecha de (6.22), escrito como un sistema de 12 ecuaciones evaluado en el punto fijo (6.23).

La estructura de dicho campo vectorial tiene la forma de la siguiente matriz cuadrada

$$
D f=\left(\begin{array}{cc}
0 & I \\
A & B
\end{array}\right)
$$

donde $I$ es la matriz identidad de tamaño $6 \times 6$, y $A$ y $B$ están dadas de la siguiente forma

$$
B=\left(\begin{array}{cccccc}
0 & 2 \Omega+2 \Omega r^{2} & 0 & 0 & 0 & 0 \\
-2 \Omega & 0 & 0 & 0 & 0 & 0 \\
0 & 0 & 0 & 2 \Omega+2 \Omega r^{2} & 0 & 0 \\
0 & 0 & -2 \Omega^{2} & 0 & 0 & 0 \\
0 & 0 & 0 & 0 & 0 & 2 \Omega \\
0 & 0 & 0 & 0 & -2 \Omega & 0
\end{array}\right)
$$




$$
A=\left(\begin{array}{cccccc}
a & 0 & b & 0 & c & 0 \\
0 & d & 0 & e & 0 & M \\
b & 0 & a & 0 & c & 0 \\
0 & e & 0 & d & 0 & M \\
-2 m & 0 & -2 m & 0 & f & 0 \\
0 & m & 0 & m & 0 & g
\end{array}\right)
$$

donde $a, b, c, d, e, f, g$ están dados por los siguientes valores

$$
\begin{aligned}
& a=\frac{6 r^{2}+3}{8\left(r^{2}+1\right)^{\frac{3}{2}}}-\frac{4 r^{2}+1}{8\left(r^{2}+1\right)^{\frac{3}{2}}}+\frac{4 m+4 r^{2} m+1}{4\left(r^{2}+1\right)^{\frac{3}{2}}}+\frac{3 r^{2}\left(4 m+4 r^{2} m+1\right)}{4\left(r^{2}+1\right)^{\frac{3}{2}}}+\frac{m\left(r^{2}+2\right)}{\sqrt{r^{2}+1}}, \\
& b=\frac{2 r^{2}+1}{8\left(r^{2}+1\right)^{\frac{3}{2}}}-\frac{6 r^{2}+3}{8\left(r^{2}+1\right)^{\frac{3}{2}}}, \\
& c=-m\left(2 r^{2}+2\right), \\
& d=\frac{4 m+4 r^{2} m+1}{4\left(r^{2}+1\right)^{\frac{3}{2}}}-\frac{2 r^{2}+1}{8\left(r^{2}+1\right)^{\frac{3}{2}}}-m \sqrt{r^{2}+1}+\frac{r^{2}\left(4 m+4 r^{2} m+1\right)}{4\left(r^{2}+1\right)^{\frac{3}{2}}}, \\
& e=\frac{1}{8\left(r^{2}+1\right)^{\frac{3}{2}}}, \\
& f=4 \sqrt{r^{2}+1}+\frac{4 m+4 r^{2} m+1}{4\left(r^{2}+1\right)^{\frac{3}{2}}}, \\
& g=\frac{4 m+4 r^{2} m+1}{4\left(r^{2}+1\right)^{\frac{3}{2}}}-2 \sqrt{r^{2}+1} .
\end{aligned}
$$

Recordemos que para la existencia que equilibrios relativos la velocidad angular debe cumplir

$$
\Omega^{2}=\frac{4 M\left(1+r^{2}\right)+m}{4\left(1+r^{2}\right)^{3 / 2}} .
$$

Consideremos masas iguales a 1. La matriz $D f$ la podemos reescribir como 


$$
D f=\left(\begin{array}{cc}
0 & I \\
A & \Omega \widehat{B}
\end{array}\right)
$$

con

$$
\widehat{B}=\left(\begin{array}{cccccc}
0 & 2+2 r^{2} & 0 & 0 & 0 & 0 \\
-2 & 0 & 0 & 0 & 0 & 0 \\
0 & 0 & 0 & 2+2 r^{2} & 0 & 0 \\
0 & 0 & -2 & 0 & 0 & 0 \\
0 & 0 & 0 & 0 & 0 & 2 \\
0 & 0 & 0 & 0 & -2 & 0
\end{array}\right)
$$

Los vectores propios de $D f$ son los ceros de la ecuación $\operatorname{det}(D f-\lambda I)=0$. Definamos $\mu$ tal que $\lambda=\Omega \mu$, por lo que buscaremos los ceros de la ecuación $\operatorname{det}(D f-\Omega \mu I)=0$. La condicón que buscaremos para los valores propios puede ser escrita como

$$
\left(\begin{array}{cc}
0 & I \\
A & \Omega \widetilde{B}
\end{array}\right)\left(\begin{array}{l}
u \\
v
\end{array}\right)=\Omega \mu\left(\begin{array}{l}
u \\
v
\end{array}\right),
$$

donde $u, v$ están formados por las primeras y últimas cuatro componentes, respectivamente, del eigenvector correspondiente al eigenvalor $\Omega \mu$.

Así la condición del eigenvalor con parámetro $\mu$ se sigue de

$$
v=\Omega \mu u, \quad A u+\Omega \widetilde{B} \Omega \mu u=\Omega \mu v=\Omega^{2} \mu^{2} u .
$$

Denotemos al polinomio caracteristico como $p(\mu)$. La condición de valor propio debe satisfacer

$$
p(\mu)=\operatorname{det}\left(A+\Omega^{2} \mu \widetilde{B}-\Omega^{2} \mu^{2} I\right)=0 .
$$

Si denotamos a $r^{2}$ por $R$ entonces

$$
\left.\begin{array}{cccccc}
\multicolumn{7}{c}{A+\Omega^{2} \mu \widetilde{B}-\Omega^{2} \mu^{2} I=} \\
\left(\begin{array}{ccccc}
c \\
f_{1}
\end{array}\right. & g_{1} & g_{2} & 0 & -m(2 R+2) & 0 \\
-\frac{\mu(8 m+8 R m+2)}{4(R+1)^{\frac{3}{2}}} & f_{2} & 0 & \frac{1}{8(R+1)^{\frac{3}{2}}} & 0 & m \\
g_{2} & 0 & f_{1} & g_{1} & -m(2 R+2) & 0 \\
0 & 1 & -\frac{\mu(8 m+8 R m+2)}{4(R+1)^{\frac{3}{2}}} & f_{2} & 0 & m \\
-2 & 0 & -2 & 0 & f_{3} & \frac{\mu(8 m+8 R m+2)}{4(R+1)^{\frac{3}{2}}} \\
0 & 1 & 0 & 1 & -\frac{\mu(8 m+8 R m+2)}{4(R+1)^{\frac{3}{2}}} & f_{4}
\end{array}\right),
$$

donde 


$$
\begin{aligned}
f_{1}= & \frac{6 R+3}{8(R+1)^{\frac{3}{2}}}-\frac{4 R+1}{8(R+1)^{\frac{3}{2}}}+\frac{4 m+4 R m+1}{4(R+1)^{\frac{3}{2}}}+\frac{3 R(4 m+4 R m+1)}{4(R+1)^{\frac{3}{2}}} \\
& -\frac{\mu^{2}(4 m+4 R m+1)}{4(R+1)^{\frac{3}{2}}+\frac{m(R+2)}{\sqrt{R+1}},} \\
f_{2}= & \frac{4 m+4 R m+1}{4(R+1)^{\frac{3}{2}}}-\frac{2 R+1}{8(R+1)^{\frac{3}{2}}}-m \sqrt{R+1}+\frac{R(4 m+4 R m+1)}{4(R+1)^{\frac{3}{2}}} \\
& -\frac{\mu^{2}(4 m+4 R m+1)}{4(R+1)^{\frac{3}{2}}}, \\
f_{3}= & 4 \sqrt{R+1}+\frac{4 m+4 R m+1}{4(R+1)^{\frac{3}{2}}}-\frac{\mu^{2}(4 m+4 R m+1)}{4(R+1)^{\frac{3}{2}}}, \\
f_{4}= & \frac{4 m+4 R m+1}{4(R+1)^{\frac{3}{2}}-2 \sqrt{R+1}-\frac{\mu^{2}(4 m+4 R m+1)}{4(R+1)^{\frac{3}{2}}},} \\
g_{1}= & \frac{\mu(2 R+2)(4 m+4 R m+1)}{4(R+1)^{\frac{3}{2}}}, \\
g_{2}= & \frac{2 R+1}{8(R+1)^{\frac{3}{2}}}-\frac{6 R+3}{8(R+1)^{\frac{3}{2}}} .
\end{aligned}
$$

Luego

$$
\begin{aligned}
p(\mu)= & \frac{1}{4096(R+1)^{9}} \cdot \mu^{2} \cdot\left(\mu^{2}+1\right) \cdot(4 m+4 R m+1)^{3} \cdot\left(4 m-2 R+4 R m+4 \mu^{2} m+\mu^{2}+\right. \\
& \left.4 \mu^{2} R m+1\right) \cdot\left(16 \mu^{6} R^{2} m^{2}+32 \mu^{6} R m^{2}+8 \mu^{6} R m+16 \mu^{6} m^{2}+8 \mu^{6} m+\mu^{6}\right. \\
& -32 \mu^{4} R^{3} m+32 \mu^{4} R^{2} m^{2}-88 \mu^{4} R^{2} m-8 \mu^{4} R^{2}+64 \mu^{4} R m^{2}-68 \mu^{4} R m \\
& -14 \mu^{4} R+32 \mu^{4} m^{2}-12 \mu^{4} m-5 \mu^{4}-128 \mu^{2} R^{4} m-128 \mu^{2} R^{4}-512 \mu^{2} R^{3} m \\
& -528 \mu^{2} R^{3}+16 \mu^{2} R^{2} m^{2}-744 \mu^{2} R^{2} m-800 \mu^{2} R^{2}+32 \mu^{2} R m^{2}-464 \mu^{2} R m \\
& -524 \mu^{2} R+16 \mu^{2} m^{2}-104 \mu^{2} m-125 \mu^{2}-256 R^{5}-128 R^{4} m-1152 R^{4}-480 R^{3} m \\
& \left.-2032 R^{3}-656 R^{2} m-1752 R^{2}-388 R m-734 R-84 m-119\right) .
\end{aligned}
$$

Cuando tomamos $m \rightarrow 0$ obtenemos

$$
\begin{aligned}
p(\mu)= & \frac{1}{4096(R+1)^{9}} \mu^{2} \cdot\left(\mu^{2}+1\right) \cdot\left(\mu^{2}-2 R+1\right) \cdot\left(\mu^{2}+2 R+1\right) \cdot\left(\mu^{4}-8 \mu^{2} R^{2}\right. \\
& \left.-16 \mu^{2} R-6 \mu^{2}-128 R^{4}-512 R^{3}-760 R^{2}-496 R-119\right) .
\end{aligned}
$$

Notemos que el factor

$$
\left(M^{4}-8 M^{2} R^{2}-16 M^{2} R-6 M^{2}-128 R^{4}-512 R^{3}-760 R^{2}-496 R-119\right)
$$


tiene un cambio de signo, entonces por la regla de los signos de Descartes el factor tiene una raíz real positiva, misma que es raíz real positiva de $p(\mu)$. Luego las órbitas generadas son inestables.

OBSERVACIÓN 2 Al igual que en $S^{2}$ mostramos que tenemos diferentes resultados acerca de estabilidad e inestabilidad del problema de dos cuerpos, dependiendo del espacio fase en que se exponga el problema. Si consideramos el problema en donde las masas iguales giran uniformemente de forma paralela al plano xy entonces existe un intervalo sobre el eje $z$ en donde las órbitas son estables, y un intervalo en donde son inestables; si consideramos el problema de dos cuerpos en donde las partículas de masas iguales giran de forma paralela al eje xy y además existe otra partícula de masa $m$ fija en el punto $(0,0,1)$, si tomamos el límite $m \rightarrow 0$, entonces las órbitas generadas son inestables. Es claro que este caso es también un problema de dos cuerpos en $\mathrm{H}^{2}$, esto es lo que hemos explicado en los teoremas (6.1.1) y (6.1.2).

La situación es la misma que en $S^{2}$, la razón por la que tenemos diferentes resultados es que el problema está planteado en distintos contextos. Cuando consideramos sólo dos partículas, como en el primer caso, el problema está formulado en el espacio fase

$$
\left(\mathbb{R}^{2 n} \backslash \triangle\right) \times T \mathbb{R}^{2 n} \subseteq \mathbb{R}^{8},
$$

donde $T \mathbb{R}^{2 n}$ es el haz tangente de $\mathbb{R}^{2 n},(n=2)$.

Si consideramos tres partículas, como en el segundo caso (una de masa $m$ en el punto $(0,0,1))$ el problema está planteado en el espacio fase

$$
\left(\mathbb{R}^{2 n} \backslash \triangle\right) \times T \mathbb{R}^{2 n} \subseteq \mathbb{R}^{12},
$$

donde $T \mathbb{R}^{2 n}$ es el haz tangente de $\mathbb{R}^{2 n},(n=3)$.

\subsubsection{Masas Iguales}

A continuación mostramos un resultado importante que obtuvimos para los equilibrios relativos eulerianos con masas iguales

Teorema 6.1.3 Consideremos órbitas eulerianas periódicas del problema curvado de tres cuerpos en $H^{2}$, con masas $m_{1}=m_{2}=m_{3}$, en donde $m_{1}$ y $m_{2}$ se encuentran fijas en los lados opuestos de un círculo que rota uniformemente y de forma paralela al plano xy, y la partícula $m_{3}$ fija en el punto $(0,0,1)$, entonces para cualquier $z>1$ las órbitas generadas son inestables. 


\section{Demostración}

En esta clase de equilibrios relativos introduciremos, como en $S^{2}$, coordenadas rotatorias. Tomemos masas normalizadas, es decir $m_{1}=m_{2}=m_{3}=1$, y consideremos los siguientes cambios de coordenadas y reescalamiento de tiempo para simplificar cálculos en las variables.

$$
\begin{gathered}
x_{i}=r X_{i}, \quad y_{i}=r Y_{i}, \quad r \in(0, \infty), \\
t=r^{\frac{3}{2}} \tau,
\end{gathered}
$$

tendremos entonces las ecuaciones de movimiento en términos de

$$
Q=(X, Y, z) .
$$

Hacemos tambien un reescalamiento sobre el valor de la velocidad angular, $\Omega=\frac{\omega t}{\tau}$. De esta manera tenemos

$$
\begin{aligned}
\Omega^{2} & =\frac{\omega^{2} t^{2}}{\tau^{2}} \\
& =\frac{\omega^{2} r^{3} \tau^{2}}{\tau^{2}} \\
& =\omega^{2} r^{3} \\
& =\frac{4 m_{3} z^{2}+m_{1}}{4 z^{3}\left(z^{2}-1\right)} r^{3} \\
& =\frac{m_{1}+4 m_{3}\left(1+r^{2}\right)}{4\left(1+r^{2}\right)^{\frac{3}{2}}} .
\end{aligned}
$$

De forma análoga que en $S^{2}$ tenemos

$$
\begin{aligned}
X_{i}^{\prime} & =r^{\frac{1}{2}} \dot{x}_{i}, \\
X_{i}^{\prime \prime} & =r^{2} \ddot{x}_{i}, \\
Y_{i}^{\prime} & =r^{\frac{1}{2}} \dot{y}_{i}, \\
Y_{i}^{\prime \prime} & =r^{2} \ddot{y}_{i},
\end{aligned}
$$

para $i=1,2,3$. 
Ahora aplicamos las coordenadas reescaladas a las ecuaciones de movimiento, teniendo

$$
\begin{aligned}
\ddot{q}_{i} & =\sum_{j=1, j \neq i}^{3} \frac{m_{j}\left(q_{j}+\left(q_{i} \odot q_{j}\right) q_{i}\right)}{\left(\left(q_{i} \odot q_{j}\right)^{2}-1\right)^{\frac{3}{2}}}+\left(\dot{q}_{i} \odot \dot{q}_{i}\right) q_{i} \\
& =\sum_{j=1, j \neq i}^{3} \frac{m_{j}\left(\left(x_{j}, y_{j}, z_{j}\right)+\left(\left(x_{i}, y_{i}, z_{i}\right) \odot\left(x_{j}, y_{j}, z_{j}\right)\right)\left(x_{i}, y_{i}, z_{i}\right)\right)}{\left(\left(\left(x_{i}, y_{i}, z_{i}\right) \odot\left(x_{j}, y_{j}, z_{j}\right)\right)^{2}-1\right)^{\frac{3}{2}}} \\
& +\left(\left(\dot{x}_{i}, \dot{y}_{i}, \dot{z}_{i}\right) \odot\left(\dot{x}_{i}, \dot{y}_{i}, \dot{z}_{i}\right)\right)\left(x_{i}, y_{i}, z_{i}\right) \\
& =\sum_{j=1, j \neq i}^{3} \frac{m_{j}\left(\left(r X_{j}, r Y_{j}, z_{j}\right)+\left(\left(x_{i}, y_{i}, z_{i}\right) \odot\left(x_{j}, y_{j}, z_{j}\right)\right)\left(r X_{i}, r Y_{i}, z_{i}\right)\right)}{\left(\left(\left(x_{i}, y_{i}, z_{i}\right) \odot\left(x_{i}, y_{i}, z_{i}\right)\right)^{2}-1\right)^{\frac{3}{2}}} \\
& +\left(\left(\dot{x}_{i}, \dot{y}_{i}, \dot{z}_{i}\right) \odot\left(\dot{x}_{i}, \dot{y}_{i}, \dot{z}_{i}\right)\right)\left(r X_{i}, r Y_{i}, z_{i}\right),
\end{aligned}
$$

para $i=1,2,3$.

En los equilibrios relativos elipticos las órbitas giran de forma paralela al plano $z=0$, entonces las coorrespondientes coordenadas $z_{i}$ de las partículas se mantienen constantes, por lo que la omitiremos en el análisis.

Introducimos coordenadas rotatorias $\left(\xi_{i}, \eta_{i}\right), i=1,2,3$,

$$
\left(\begin{array}{c}
X_{i} \\
Y_{i}
\end{array}\right)=R(\Omega \tau)\left(\begin{array}{c}
\xi_{i} \\
\eta_{i}
\end{array}\right)
$$

en donde $R(\Omega \tau)$ es la matriz de rotación

$$
R(\Omega \tau)=\left(\begin{array}{cc}
\cos \Omega \tau & -\sin \Omega \tau \\
\sin \Omega \tau & \cos \Omega \tau
\end{array}\right)
$$

Reescribiendo las ecuaciones de movimiento en términos de $\left(\xi_{i}, \eta_{i}\right)$ como lo hicimos en el caso de $S^{2}$ obtenemos las ecuaciones de movimiento en $H^{2}$ en un sistema rotatorio que gira a velocidad $\Omega$ 


$$
\begin{aligned}
\left(\begin{array}{c}
\xi_{i}^{\prime \prime} \\
\eta_{i}^{\prime \prime}
\end{array}\right) & =2 \Omega\left(\begin{array}{c}
\eta_{i}^{\prime} \\
-\xi_{i}^{\prime}
\end{array}\right)+\Omega^{2}\left(\begin{array}{c}
\xi_{i} \\
\eta_{i}
\end{array}\right)+r^{2} h_{i}\left(\begin{array}{c}
\xi_{i} \\
\eta_{i}
\end{array}\right) \\
& +\sum_{j=1, j \neq i}^{3} m_{j}\left[\xi_{i}^{2}+\eta_{i}^{2}+\xi_{j}^{2}+\eta_{j}^{2}-2\left(\xi_{i} \xi_{j}+\eta_{i} \eta_{j}\right) \sqrt{\left(1+r^{2}\left(\xi_{i}^{2}+\eta_{i}^{2}\right)\right)\left(1+r^{2}\left(\xi_{j}^{2}+\eta_{j}^{2}\right)\right)}\right. \\
& \left.+r^{2}\left(\left(\xi_{i} \xi_{j}+\eta_{i} \eta_{j}\right)^{2}+\left(\xi_{i}^{2}+\eta_{i}^{2}\right)\left(\xi_{j}^{2}+\eta_{j}^{2}\right)\right)\right]^{-3 / 2}\left[\left(\begin{array}{c}
\xi_{j} \\
\eta_{j}
\end{array}\right)+\left(r^{2}\left(\xi_{i} \xi_{j}+\eta_{i} \eta_{j}\right)\right.\right. \\
& \left.\left.-\sqrt{\left(1+r^{2}\left(\xi_{i}^{2}+\eta_{i}^{2}\right)\right)\left(1+r^{2}\left(\xi_{j}^{2}+\eta_{j}^{2}\right)\right)}\right)\left(\begin{array}{c}
\xi_{i} \\
\eta_{i}
\end{array}\right)\right]
\end{aligned}
$$

donde

$$
h_{i}=\Omega^{2}\left(\xi_{i}^{2}+\eta_{i}^{2}\right)+2 \Omega\left(\xi_{i} \eta_{i}^{\prime}-\eta_{i} \xi_{i}^{\prime}\right)+\left(\left(\xi_{i}^{\prime 2}\right)+\left(\eta_{i}^{\prime 2}\right)\right)-\frac{r^{2}}{1+r^{2}\left(\xi_{i}^{2}+\eta_{i}^{2}\right)}\left(\xi_{i} \xi_{i}^{\prime}+\eta_{i} \eta_{i}^{\prime}\right)^{2},
$$

para $i=1,2,3$.

En (6.48) comprobamos que

$\xi_{1}=1, \eta_{1}=0, \xi_{2}=-1, \eta_{2}=0, \xi_{3}=0, \eta_{3}=0, \xi_{1}^{\prime}=0, \eta_{1}^{\prime}=0, \xi_{2}^{\prime}=0, \eta_{2}^{\prime}=0, \xi_{3}^{\prime}=0, \eta_{3}^{\prime}=0$ es punto fijo del sistema.

Estudiaremos los eigenvalores del campo vectorial de la diferencial de la función de la parte derecha de (6.48), escrito como un sistema de 12 ecuaciones evaluado en el punto fijo (6.49).

La estructura de dicho campo vectorial tiene la forma de la siguiente matriz cuadrada

$$
D f=\left(\begin{array}{cc}
0 & I \\
A & B
\end{array}\right)
$$

donde $I$ es la matriz identidad de tamaño $6 \times 6$, y $A$ y $B$ están dadas de la siguiente forma

$$
B=\left(\begin{array}{cccccc}
0 & 2 \Omega+2 \Omega r^{2} & 0 & 0 & 0 & 0 \\
-2 \Omega & 0 & 0 & 0 & 0 & 0 \\
0 & 0 & 0 & 2 \Omega+2 \Omega r^{2} & 0 & 0 \\
0 & 0 & -2 \Omega^{2} & 0 & 0 & 0 \\
0 & 0 & 0 & 0 & 0 & 2 \Omega \\
0 & 0 & 0 & 0 & -2 \Omega & 0
\end{array}\right),
$$




$$
A=\left(\begin{array}{cccccc}
a & 0 & b & 0 & c & 0 \\
0 & d & 0 & e & 0 & M \\
b & 0 & a & 0 & c & 0 \\
0 & e & 0 & d & 0 & M \\
-2 m & 0 & -2 m & 0 & f & 0 \\
0 & m & 0 & m & 0 & g
\end{array}\right)
$$

donde $a, b, c, d, e, f, g$ están dados por los siguientes valores

$$
\begin{aligned}
& a=\Omega^{2}+\frac{12 m}{\left(4+4 r^{2}\right)^{\frac{5}{2}}}+3 \Omega^{2} r^{2}-\frac{m\left(4 r^{2}+1\right)}{\left(4+4 r^{2}\right)^{\frac{3}{2}}}-\frac{36 m r^{2}}{\left(4+4 r^{2}\right)^{\frac{5}{2}}}+\frac{24 m r^{4}}{\left(4+4 r^{2}\right)^{\frac{5}{2}}}+\frac{M r^{2}}{(2 M+1) \sqrt{1+r^{2}}}, \\
& b=\frac{2 r^{2}+1}{\left(4+4 r^{2}\right)^{\frac{3}{2}}}-\frac{\left(6 r^{2}-6\right)\left(8 r^{2}-4\right)}{2\left(2+4 r^{2}\right)^{\frac{5}{2}}}, \\
& c=-2 M\left(r^{2}+1\right), \\
& d=\Omega^{2}-\frac{m\left(2 r^{2}-1\right)}{8\left(1+r^{2}\right)^{\frac{3}{2}}}+\Omega^{2} r^{2}-M\left(\sqrt{1+r^{2}}\right), \\
& e=\frac{1}{\left(4+4 r^{2}\right)^{\frac{3}{2}}} \\
& f=\Omega^{2}+4 m \sqrt{1+r^{2}}, \\
& g=\Omega^{2}-2 m \sqrt{1+r^{2}}+m,
\end{aligned}
$$

donde $M=m_{3}$ y $m_{1}=m_{2}=m$.

Recordemos que para la existencia que equilibrios relativos la velocidad angular debe cumplir

$$
\Omega^{2}=\frac{4 M\left(1+r^{2}\right)+m}{4\left(1+r^{2}\right)^{3 / 2}} .
$$

Consideremos masas iguales a 1 . La matriz $D f$ la podemos reescribir como 


$$
D f=\left(\begin{array}{cc}
0 & I \\
A & \Omega \widehat{B}
\end{array}\right)
$$

con

$$
\widehat{B}=\left(\begin{array}{cccccc}
0 & 2+2 r^{2} & 0 & 0 & 0 & 0 \\
-2 & 0 & 0 & 0 & 0 & 0 \\
0 & 0 & 0 & 2+2 r^{2} & 0 & 0 \\
0 & 0 & -2 & 0 & 0 & 0 \\
0 & 0 & 0 & 0 & 0 & 2 \\
0 & 0 & 0 & 0 & -2 & 0
\end{array}\right) .
$$

Los vectores propios de $D f$ son los ceros de la ecuación $\operatorname{det}(D f-\lambda I)=0$. Definamos $\mu$ tal que $\lambda=\Omega \mu$, por lo que buscaremos los ceros de la ecuación $\operatorname{det}(D f-\Omega \mu I)=0$. La condicón que buscaremos para los valores propios puede ser escrita como

$$
\left(\begin{array}{cc}
0 & I \\
A & \Omega \widetilde{B}
\end{array}\right)\left(\begin{array}{l}
u \\
v
\end{array}\right)=\Omega \mu\left(\begin{array}{l}
u \\
v
\end{array}\right),
$$

donde $u, v$ están formados por las primeras y últimas cuatro componentes, respectivamente, del eigenvector correspondiente al eigenvalor $\Omega \mu$.

Así la condición del eigenvalor con parámetro $\mu$ se sigue de

$$
v=\Omega \mu u, \quad A u+\Omega \widetilde{B} \Omega \mu u=\Omega \mu v=\Omega^{2} \mu^{2} u .
$$

Denotemos al polinomio caracteristico como $p(\mu)$. La condición de valor propio debe satisfacer

$$
p(\mu)=\operatorname{det}\left(A+\Omega^{2} \mu \widetilde{B}-\Omega^{2} \mu^{2} I\right)=0 .
$$

Si denotamos a $r^{2}$ por $R$ entonces

$$
\begin{gathered}
A+\Omega^{2} \mu \widetilde{B}-\Omega^{2} \mu^{2} I= \\
\left(\begin{array}{cccccc}
f_{1} & \frac{\mu(2 R+2)(4 R+5)}{4(R+1)^{\frac{3}{2}}} & \frac{2 R+1}{8(R+1)^{\frac{3}{2}}}-\frac{6 R+3}{8(R+1)^{\frac{3}{2}}} & 0 & -2 R-2 & 0 \\
-\frac{\mu(8 R+10)}{4(R+1)^{\frac{3}{2}}} & f_{2} & 0 & \frac{1}{8(R+1)^{\frac{3}{2}}} & 0 & 1 \\
\frac{2 R+1}{8(R+1)^{\frac{3}{2}}-\frac{6 R+3}{8(R+1)^{\frac{3}{2}}}} & 0 & f_{1} & \frac{\mu(2 R+2)(4 R+5)}{4(R+1)^{\frac{3}{2}}} & -2 R-2 & 0 \\
0 & 1 & -\frac{\mu(8 R+10)}{4(R+1)^{\frac{3}{2}}} & f_{2} & 0 & 1 \\
-2 & 0 & -2 & 0 & f_{3} & \frac{\mu(8 R+10)}{4(R+1)^{\frac{3}{2}}} \\
0 & 1 & 0 & 1 & -\frac{\mu(8 R+10)}{4(R+1)^{\frac{3}{2}}} & f_{4}
\end{array}\right),
\end{gathered}
$$


donde

$$
\begin{aligned}
& f_{1}=\frac{R+2}{\sqrt{R+1}}-\frac{4 R+1}{8(R+1)^{\frac{3}{2}}}+\frac{4 R+5}{4(R+1)^{\frac{3}{2}}}+\frac{6 R+3}{8(R+1)^{\frac{3}{2}}}-\frac{\mu^{2}(4 R+5)}{4(R+1)^{\frac{3}{2}}}+\frac{3 R(4 R+5)}{4(R+1)^{\frac{3}{2}}}, \\
& f_{2}=\frac{4 R+5}{4(R+1)^{\frac{3}{2}}}-\frac{2 R+1}{8(R+1)^{\frac{3}{2}}}-\sqrt{R+1}-\frac{\mu^{2}(4 R+5)}{4(R+1)^{\frac{3}{2}}}+\frac{R(4 R+5)}{4(R+1)^{\frac{3}{2}}} \\
& f_{3}=4 \sqrt{R+1}+\frac{4 R+5}{4(R+1)^{\frac{3}{2}}}-\frac{\mu^{2}(4 R+5)}{4(R+1)^{\frac{3}{2}}} \\
& f_{4}=\frac{4 R+5}{4(R+1)^{\frac{3}{2}}}-2 \sqrt{R+1}-\frac{\mu^{2}(4 R+5)}{4(R+1)^{\frac{3}{2}}} .
\end{aligned}
$$

En el determinante de la matriz anterior el término $\mu$ aparece en potencias pares, por lo que haremos el cambio $M=\mu^{2}$, obteniendo el polinomio $\widehat{p}(M)$, recordando que para la estabilidad espectral necesitamos raíces negativas. Después de multiplicar al polinomio $\widehat{p}$ por el factor $4096(R+1)^{9} /(4 R+5)^{3}$ obtenemos

$$
\widetilde{p}(M)=M(M+1) Q_{1}(M) Q_{2}(M),
$$

donde

$$
\begin{aligned}
Q_{1}(M)= & 2 R+4 M R+5 M+5, \\
Q_{2}(M)= & 16 M^{3} R^{2}+40 M^{3} R+25 M^{3}-32 M^{2} R^{3}-64 M^{2} R^{2}-18 M^{2} R+15 M^{2} \\
& -256 M R^{4}-1040 M R^{3}-1528 M R^{2}-956 M R-213 M-256 R^{5}-1280 R^{4} \\
& -2512 R^{3}-2408 R^{2}-1122 R-203 \\
= & \left(R^{2}+40 r+25\right) M^{3} \\
& +\left(-32 R^{3}-64 R^{2}-18 R+15\right) M^{2} \\
& +\left(-256 R^{4}-1040 R^{3}-1528 R^{2}-956 R-213\right) M \\
& -256 R^{5}-1280 R^{4}-2512 R^{3}-2408 R^{2}-1122 R-203 .
\end{aligned}
$$

El polinomio $Q_{1}(M)$ tiene raíz $M_{0}=\frac{-2 R-5}{4 R+5}<0$ para $R>0$. Analizaremos las raíces de $Q_{2}(M)$ reescribiéndolo

$$
Q_{2}(M)=g_{1}(R) M^{3}+g_{2}(R) M^{2}+g_{3}(R) M+g_{4}(R) .
$$

Es fácil notar que para $R \in(0, \infty) g_{4}(R)<0, g_{3}(R)<0$ y que $g_{1}(R)>0$, por lo que, independientemente del signo de $g_{2}(R)$, hay una variación de signo del polinomnio $Q_{2}(M)$, por lo que éste tiene una raíz real positiva (regla de los signos de Descartes), misma que es raíz del polinomio $\widehat{p}(M)$, esta raíz real positiva nos genera dos raíces reales del polinomio característico $p(\mu)$, mostrando que las órbitas que estudiamos son espectralmente inestables. 


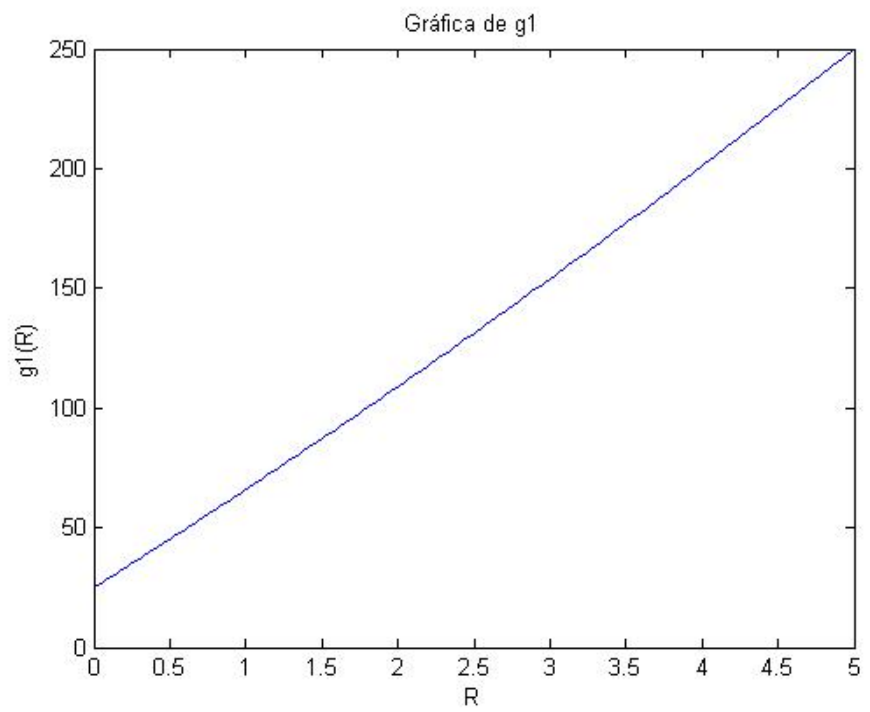

Figura 6.3: Gráfica de $g_{1}(R)$.

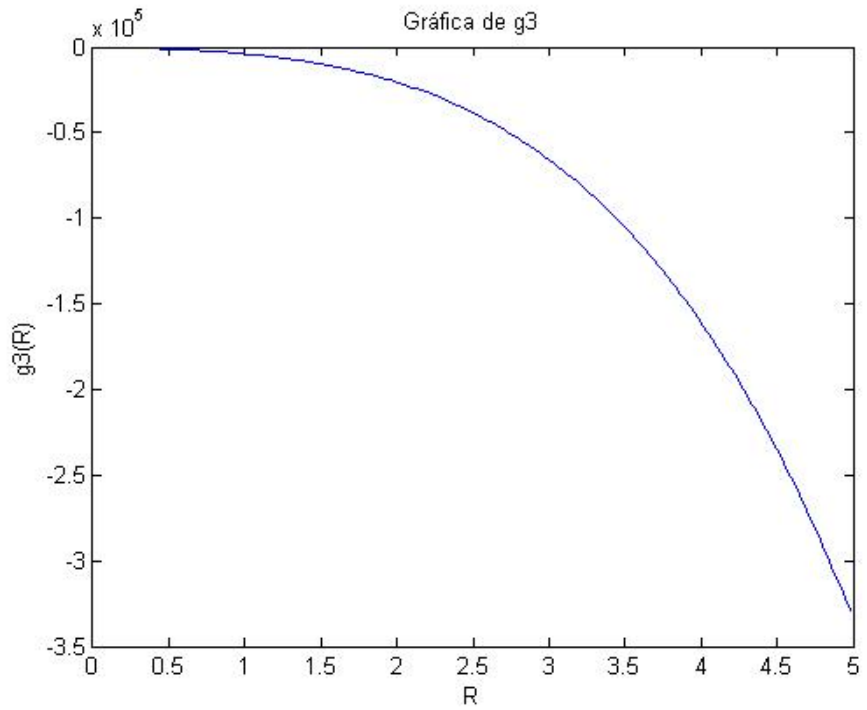

Figura 6.4: Gráfica de $g_{3}(R)$. 


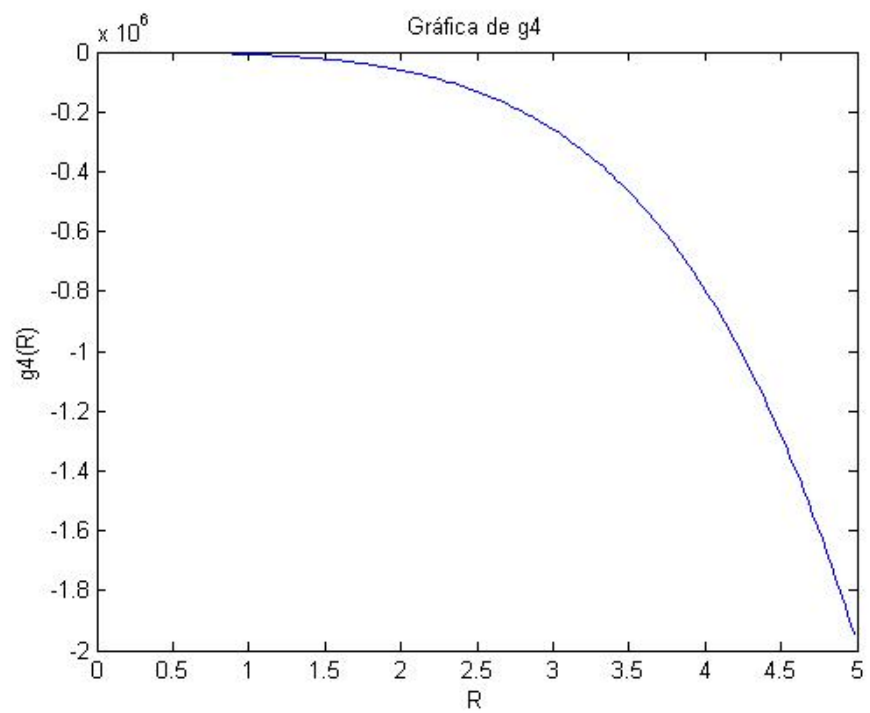

Figura 6.5: Gráfica de $g_{4}(R)$.

\subsubsection{3. $\quad 2+1$ Cuerpos}

En esta sección mostraremos el analisis que realizamos del problema $2+1$-cuerpos relacionados con los equilibrios relativos elípticos en $H^{2}$. El resultado lo mostramos en el siguiente

Teorema 6.1.4 Consideremos órbitas eulerianas elípticas del problema curvado de tres cuerpos en $\mathrm{H}^{2}$, con dos masas despreciables fijas en los lados opuestos de un círculo que rota uniformemente en un plano paralelo al plano $x y$, y una partícula de masa $m=1$ fija en el punto $(0,0,1)$, entonces para $z \in(1, \infty)$ las órbitas generadas son estables.

\section{Demostración}

Consideremos masas $m_{1} m_{2}$ despreciables en los extremos de un círculo a altura $z>1$ y masa $m_{3}$ fija en el punto $(0,0,1)$. Como las masas $m_{1}$ y $m_{2}$ suponemos que son muy pequeñas, entonces no existe fuerza de atraccíon sobre ellas y cada una de eéstas se mueve por la fuerza ejercida por el cuerpo $m_{3}$. Tenemos entonces dos sistemas desacoplados, uno formado por las masas $m_{1}$ y $m_{3}$, y otro sistema formado por $m_{2}$ y $m_{3}$.

Consideremos el sistema formado por las partículas $m_{1}$ y $m_{3}$, como anteriormente lo hicimos introducimos coordenadas rotatorias del sistema que gira a velocidad $\Omega$, aplicando la matriz de rotación a las coordenadas de las partículas

$$
\left(\begin{array}{c}
X_{i} \\
Y_{i}
\end{array}\right)=R(\Omega \tau)\left(\begin{array}{c}
\xi_{i} \\
\eta_{i}
\end{array}\right)
$$


donde $R(\Omega \tau)$ es la matriz de rotación

$$
R(\Omega \tau)=\left(\begin{array}{cc}
\cos \Omega \tau & -\sin \Omega \tau \\
\sin \Omega \tau & \cos \Omega \tau
\end{array}\right)
$$

Obtenemos las ecuaciones de movimiento en el sistema rotatorio

$$
\begin{aligned}
\left(\begin{array}{c}
\xi_{i}^{\prime \prime} \\
\eta_{i}^{\prime \prime}
\end{array}\right) & =2 \Omega\left(\begin{array}{c}
\eta_{i}^{\prime} \\
-\xi_{i}^{\prime}
\end{array}\right)+\Omega^{2}\left(\begin{array}{c}
\xi_{i} \\
\eta_{i}
\end{array}\right)+r^{2} h_{i}\left(\begin{array}{c}
\xi_{i} \\
\eta_{i}
\end{array}\right) \\
& m_{j}\left[\xi_{i}^{2}+\eta_{i}^{2}+\xi_{j}^{2}+\eta_{j}^{2}-2\left(\xi_{i} \xi_{j}+\eta_{i} \eta_{j}\right) \sqrt{\left(1+r^{2}\left(\xi_{i}^{2}+\eta_{i}^{2}\right)\right)\left(1+r^{2}\left(\xi_{j}^{2}+\eta_{j}^{2}\right)\right)}\right. \\
& \left.+r^{2}\left(\left(\xi_{i} \xi_{j}+\eta_{i} \eta_{j}\right)^{2}+\left(\xi_{i}^{2}+\eta_{i}^{2}\right)\left(\xi_{j}^{2}+\eta_{j}^{2}\right)\right)\right]^{-3 / 2}\left[\left(\begin{array}{c}
\xi_{j} \\
\eta_{j}
\end{array}\right)+\left(r^{2}\left(\xi_{i} \xi_{j}+\eta_{i} \eta_{j}\right)\right.\right. \\
& \left.\left.-\sqrt{\left(1+r^{2}\left(\xi_{i}^{2}+\eta_{i}^{2}\right)\right)\left(1+r^{2}\left(\xi_{j}^{2}+\eta_{j}^{2}\right)\right)}\right)\left(\begin{array}{c}
\xi_{i} \\
\eta_{i}
\end{array}\right)\right]
\end{aligned}
$$

donde

$$
h_{i}=\Omega^{2}\left(\xi_{i}^{2}+\eta_{i}^{2}\right)+2 \Omega\left(\xi_{i} \eta_{i}^{\prime}-\eta_{i} \xi_{i}^{\prime}\right)+\left(\left(\xi_{i}^{\prime 2}\right)+\left(\eta_{i}^{\prime 2}\right)\right)-\frac{r^{2}}{1+r^{2}\left(\xi_{i}^{2}+\eta_{i}^{2}\right)}\left(\xi_{i} \xi_{i}^{\prime}+\eta_{i} \eta_{i}^{\prime}\right)^{2},
$$

para $i=1,2$.

En el sistema podemos ver que un punto fijo es

$$
\xi_{1}=1, \eta_{1}=0, \xi_{2}=0, \eta_{2}=0 \xi_{1}^{\prime}=0, \eta_{1}^{\prime},=0, \xi_{2}^{\prime}=0, \eta_{2}^{\prime}=0 .
$$

La matriz $D f$ correspondiente es una matriz de tamaño $8 \times 8$ de la forma

$$
D f=\left(\begin{array}{cc}
0 & I \\
A & B
\end{array}\right)
$$

en donde $I$ es la matriz identidad de tamaño $4 \times 4$. La matriz $A$ en este caso tiene la forma

$$
A=\left(\begin{array}{llll}
a & 0 & b & 0 \\
0 & c & 0 & d \\
b & 0 & a & 0 \\
0 & d & 0 & c
\end{array}\right)
$$

donde 


$$
\begin{aligned}
& a=\frac{r^{2}}{\sqrt{r^{2}+1}}+\Omega^{2}+3 \Omega^{2} r^{2}+2 \sqrt{r^{2}+1}, \\
& b=-2 r^{2}-2 \\
& c=\Omega^{2}+\Omega^{2} r^{2}-\sqrt{r^{2}+1} \\
& d=1 .
\end{aligned}
$$

La matriz $B$

$$
B=\left(\begin{array}{rrrr}
0 & 2 \Omega-2 \Omega r^{2} & 0 & 0 \\
-2 \Omega & 0 & 0 & 0 \\
0 & 0 & 0 & 2 \Omega \\
0 & 0 & -2 \Omega & 0
\end{array}\right)
$$

En este problema la velodidad angular debe cumplir

$$
\Omega^{2}=\frac{4\left(1-r^{2}\right)+m}{4\left(1-r^{2}\right)^{3 / 2}} .
$$

Los vectores propios de $D f$ son los ceros de la ecuación $\operatorname{det}(D f-\lambda I)=0$. Definamos $\mu$ tal que $\lambda=\Omega \mu$, por lo que buscaremos los ceros de la ecuación $\operatorname{det}(D f-\Omega \mu I)=0$. La condicón que buscaremos para los valores propios puede ser escrita como

$$
\left(\begin{array}{cc}
0 & I \\
A & \Omega \widetilde{B}
\end{array}\right)\left(\begin{array}{l}
u \\
v
\end{array}\right)=\Omega \mu\left(\begin{array}{l}
u \\
v
\end{array}\right),
$$

donde $u, v$ están formados por las primeras y últimas cuatro componentes, respectivamente, del eigenvector correspondiente al eigenvalor $\Omega \mu$.

Así la condición del eigenvalor con parámetro $\mu$ se sigue de

$$
v=\Omega \mu u, \quad A u+\Omega \widetilde{B} \Omega \mu u=\Omega \mu v=\Omega^{2} \mu^{2} u .
$$

Denotemos al polinomio caracteristico como $p(\mu)$. La condición de valor propio debe satisfacer

$$
p(\mu)=\operatorname{det}\left(A+\Omega^{2} \mu \widetilde{B}-\Omega^{2} \mu^{2} I\right)=0 .
$$

Si denotamos a $r^{2}$ por $R$ entonces

$$
\begin{aligned}
& A+\Omega^{2} \mu \widetilde{B}-\Omega^{2} \mu^{2} I= \\
& \left.\qquad \begin{array}{cccc}
f_{1} & \mu\left(\frac{m+4 R+4}{2(R+1)^{\frac{3}{2}}}+\frac{R(m+4 R+4)}{2(R+1)^{\frac{3}{2}}}\right) & -2 R-2 & 0 \\
-\frac{\mu(m+4 R+4)}{2(R+1)^{\frac{3}{2}}} & f_{2} & 0 & 1 \\
-2 m & 0 & f_{3} & \frac{\mu(m+4 R+4)}{2(R+1)^{\frac{3}{2}}} \\
0 & m & -\frac{\mu(m+4 R+4)}{2(R+1)^{\frac{3}{2}}} & f_{4}
\end{array}\right),
\end{aligned}
$$


donde

$$
\begin{aligned}
& f_{1}=2 \sqrt{R+1}-\frac{R}{\sqrt{R+1}}+\frac{m+4 R+4}{4(R+1)^{\frac{3}{2}}}+\frac{3 R(m+4 R+4)}{4(R+1)^{\frac{3}{2}}}-\frac{\mu^{2}(m+4 R+4)}{4(R+1)^{\frac{3}{2}}}, \\
& f_{2}=\frac{m+4 R+4}{4(R+1)^{\frac{3}{2}}}-\sqrt{R+1}+\frac{R(m+4 R+4)}{4(R+1)^{\frac{3}{2}}}-\frac{\mu^{2}(m+4 R+4)}{4(R+1)^{\frac{3}{2}}}, \\
& f_{3}=2 m \sqrt{R+1}+\frac{m+4 R+4}{4(R+1)^{\frac{3}{2}}}-\frac{\mu^{2}(m+4 R+4)}{4(R+1)^{\frac{3}{2}}}, \\
& f_{4}=\frac{m+4 R+4}{4(R+1)^{\frac{3}{2}}}-m \sqrt{R+1}-\frac{\mu^{2}(m+4 R+4)}{4(R+1)^{\frac{3}{2}}} .
\end{aligned}
$$

Cuyo determinante es

$$
\begin{aligned}
& p(\mu)=-\frac{1}{256(R+1)^{6}}\left(96 m^{4} R^{6}+512 m^{4} R^{5}+32 m^{4} R^{4} \mu^{4}+76 m^{4} R^{4} \mu^{2}+1108 m^{4} R^{4}\right. \\
& +128 m^{4} R^{3} \mu^{4}+296 m^{4} R^{3} \mu^{2}+1240 m^{4} R^{3}+4 m^{4} R^{2} \mu^{6}+193 m^{4} R^{2} \mu^{4} \\
& +418 m^{4} R^{2} \mu^{2}+749 m^{4} R^{2}+8 m^{4} R \mu^{6}+132 m^{4} R \mu^{4}+256 m^{4} R \mu^{2} \\
& +228 m^{4} R-m^{4} \mu^{8}+30 m^{4} \mu^{4}+56 m^{4} \mu^{2}+27 m^{4}+256 M^{3} R^{7}+256 M^{3} R^{6} \mu^{2} \\
& +2048 m^{3} R^{6}+256 m^{3} R^{5} \mu^{4}+1840 m^{3} R^{5} \mu^{2}+6608 m^{3} R^{5}+1344 m^{3} R^{4} \mu^{4} \\
& +5456 m^{3} R^{4} \mu^{2}+11376 m^{3} R^{4}+48 m^{3} R^{3} \mu^{6}+2824 m^{3} R^{3} \mu^{4}+8400 m^{3} R^{3} \mu^{2} \\
& +11352 m^{3} R^{3}+144 m^{3} R^{2} \mu^{6}+2988 m^{3} R^{2} \mu^{4}+7064 m^{3} R^{2} \mu^{2}+6556 m^{3} R^{2} \\
& -16 m^{3} R \mu^{8}+84 m^{3} R \mu^{6}+1528 m^{3} R \mu^{4}+3060 m^{3} R \mu^{2}+2016 m^{3} R-16 m^{3} \mu^{8} \\
& -12 m^{3} \mu^{6}+276 m^{3} \mu^{4}+524 m^{3} \mu^{2}+252 m^{3}+1024 m^{2} R^{7} \mu^{2}+1024 m^{2} R^{7} \\
& +512 m^{2} R^{6} \mu^{4}+7168 m^{2} R^{6} \mu^{2}+6656 m^{2} R^{6}+3584 m^{2} R^{5} \mu^{4}+22528 m^{2} R^{5} \mu^{2} \\
& +18944 m^{2} R^{5}+192 m^{2} R^{4} \mu^{6}+10256 m^{2} R^{4} \mu^{4}+40416 m^{2} R^{4} \mu^{2}+30352 m^{2} R^{4} \\
& +768 m^{2} R^{3} \mu^{6}+15488 m^{2} R^{3} \mu^{4}+44032 m^{2} R^{3} \mu^{2}+29312 m^{2} R^{3}-96 m^{2} R^{2} \mu^{8} \\
& +816 m^{2} R^{2} \mu^{6}+12704 m^{2} R^{2} \mu^{4}+28720 m^{2} R^{2} \mu^{2}+16928 m^{2} R^{2}-192 m^{2} R \mu^{8} \\
& +96 m^{2} R \mu^{6}+5120 m^{2} R \mu^{4}+10208 m^{2} R \mu^{2}+5376 m^{2} R-96 m^{2} \mu^{8} \\
& -144 m^{2} \mu^{6}+720 m^{2} \mu^{4}+1488 m^{2} \mu^{2}+720 m^{2}+1024 m R^{6} \mu^{4}+2048 m R^{6} \mu^{2} \\
& +1024 m R^{6}+256 m R^{5} \mu^{6}+6144 m R^{5} \mu^{4}+11520 m R^{5} \mu^{2}+5632 m R^{5} \\
& +1280 m R^{4} \mu^{6}+15424 m R^{4} \mu^{4}+27008 m R^{4} \mu^{2}+12864 m R^{4}-256 m R^{3} \mu^{8} \\
& +1728 m R^{3} \mu^{6}+19840 m R^{3} \mu^{4}+33472 m R^{3} \mu^{2}+15616 m R^{3}-768 m R^{2} \mu^{8} \\
& +64 m R^{2} \mu^{6}+13056 m R^{2} \mu^{4}+22848 m R^{2} \mu^{2}+10624 m R^{2}-768 m R \mu^{8} \\
& -1216 m R \mu^{6}+3712 m R \mu^{4}+8000 m R \mu^{2}+3840 m R-256 m \mu^{8}-576 m \mu^{6}
\end{aligned}
$$




$$
\begin{aligned}
& +192 m \mu^{4}+1088 m \mu^{2}+576 m-256 R^{4} \mu^{8}-768 R^{4} \mu^{6}-768 R^{4} \mu^{4}-256 R^{4} \mu^{2} \\
& -1024 R^{3} \mu^{8}-3072 R^{3} \mu^{6}-3072 R^{3} \mu^{4}-1024 R^{3} \mu^{2}-1536 R^{2} \mu^{8}-4608 R^{2} \mu^{6} \\
& -4608 R^{2} \mu^{4}-1536 R^{2} \mu^{2}-1024 R \mu^{8}-3072 R \mu^{6}-3072 R \mu^{4}-1024 R \mu^{2}-256 \mu^{8} \\
& \left.-768 \mu^{6}-768 \mu^{4}-256 \mu^{2}\right) .
\end{aligned}
$$

Suponemos que la masa $m$ es despreciable, entonces tomando $\operatorname{lím}_{m \rightarrow 0} p(\mu)$, obtenemos

$$
p(\mu)=\frac{\mu^{2}\left(\mu^{2}+1\right)^{3}}{(R+1)^{2}},
$$

mostrando que las órbitas generadas son estables, ya que tenemos raíces del polinomio característico $p(\mu)$ que son puramente complejas e iguales a cero.

\subsection{Equilibrios Relativos Hiperbólicos}

Ahora pasamos al siguiente caso que es el estudio de equilibrios relativos eulerianos del tipo hiperbólico, recordemos que estos son soluciones de las ecuaciones de movimiento en donde las partículas se mueven a lo largo de hipérbolas, esta definición la mostramos en la sección (4.1). Mostraremos resultados sobre el problema de dos y tres cuerpos.

\subsubsection{Problema de Dos Cuerpos}

En esta sección mostraremos resultados sobre el problema de dos cuerpos, en donde estos se encuentran moviéndose sobre hipérbolas sobre $H^{2}$, en planos perpendiculares al plano $x y$, ver figura (6.6). Comencemos con el análisis del problema de dos cuerpos con el siguiente teorema. 


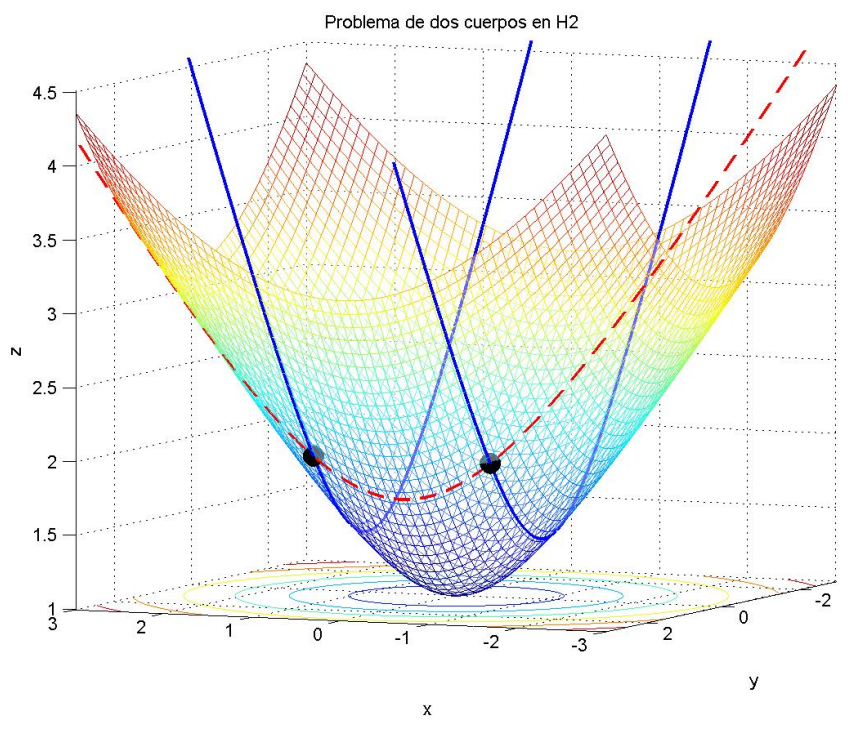

Figura 6.6: Equilibrios relativos hiperbólicos del problema de dos cuerpos en $H^{2}$. Los cuerpos se encuentran moviéndose sobre hipérbolas con $x= \pm$ cte (curvas continuas-azules), y manteniendo la misma distancia, en todo tiempo, medida sobre geodésicas (curva punteada-roja).

Teorema 6.2.1 Consideremos órbitas eulerianas del problema curvado de los dos cuerpos con masas iguales en $H^{2}$, donde el cuerpo $m_{i}$ tiene coordenadas $q_{i}=\left(x_{i}, y_{i}, z_{i}\right)$. Si las masas $m_{1}$ y $m_{2}$ se mueven a lo largo de hipérbolas de la forma $x_{1}=$ cte, $x_{2}=-x_{1}, y_{1}=y_{2}=$ $r \sinh (t), z_{1}=z_{2}=r \cosh (t), t \in \mathbb{R}$, entonces las órbitas generadas son inestables para cualquier $r>1$.

\section{Demostración}

Para estudiar la estabilidad de equilibrios relativos hiperbólicos introduciremos coordenadas rotatorias. Tomemos en cuenta valores de masas normalizadas. Además consideremos los siguientes cambios de coordenadas y reescalamiento de tiempo, este último para simplificar el valor de $\omega$.

$$
\begin{aligned}
y_{i} & =r Y_{i}, \quad z_{i}=r Z_{i}, \quad r \in(1, \infty), \\
t & =r^{\frac{3}{2}} \tau, \\
Q & =(Y, Z) .
\end{aligned}
$$

Hacemos tambien un reescalamiento sobre el valor de la velocidad angular, $\Omega=\frac{\omega t}{\tau}$. De esta manera tenemos 


$$
\begin{aligned}
\Omega^{2} & =\frac{\omega^{2} t^{2}}{\tau^{2}} \\
& =\frac{\omega^{2} r^{3} \tau^{2}}{\tau^{2}} \\
& =\omega^{2} r^{3} \\
& =\frac{1}{4 x^{2}|x|\left(x^{2}+1\right)^{\frac{3}{2}}} r^{3} \\
& =\frac{1}{4\left(r^{2}-1\right)^{\frac{3}{2}}} .
\end{aligned}
$$

Introducimos la notación

$$
\begin{aligned}
Y_{i}^{\prime} & =r^{\frac{1}{2}} \dot{y}_{i}, \\
Y_{i}^{\prime \prime} & =r^{2} \ddot{y}_{i}, \\
Z_{i}^{\prime} & =r^{\frac{1}{2}} \dot{z}_{i}, \\
Z_{i}^{\prime \prime} & =r^{2} \ddot{z}_{i},
\end{aligned}
$$

para $i=1,2$.

Ahora aplicamos las coordenadas reescaladas a las ecuaciones de movimiento, teniendo

$$
\begin{aligned}
\ddot{q}_{i} & =\sum_{j=1, j \neq i}^{3} \frac{m_{j}\left(q_{j}+\left(q_{i} \odot q_{j}\right) q_{i}\right)}{\left(\left(q_{i} \odot q_{j}\right)^{2}-1\right)^{\frac{3}{2}}}+\left(\dot{q}_{i} \odot \dot{q}_{i}\right) q_{i} \\
& =\sum_{j=1, j \neq i}^{3} \frac{m_{j}\left(\left(x_{j}, y_{j}, z_{j}\right)+\left(\left(x_{i}, y_{i}, z_{i}\right) \odot\left(x_{j}, y_{j}, z_{j}\right)\right)\left(x_{i}, y_{i}, z_{i}\right)\right)}{\left(\left(\left(x_{i}, y_{i}, z_{i}\right) \odot\left(x_{j}, y_{j}, z_{j}\right)\right)^{2}-1\right)^{\frac{3}{2}}} \\
& +\left(\left(\dot{x}_{i}, \dot{y}_{i}, \dot{z}_{i}\right) \odot\left(\dot{x}_{i}, \dot{y}_{i}, \dot{z}_{i}\right)\right)\left(x_{i}, y_{i}, z_{i}\right) \\
& =\sum_{j=1, j \neq i}^{3} \frac{m_{j}\left(\left(x_{j}, r Y_{j}, r Z_{j}\right)+\left(\left(x_{i}, y_{i}, z_{i}\right) \odot\left(x_{j}, y_{j}, z_{j}\right)\right)\left(x_{i}, r Y_{i}, r Z_{i}\right)\right)}{\left(\left(\left(x_{i}, y_{i}, z_{i}\right) \odot\left(x_{i}, y_{i}, z_{i}\right)\right)^{2}-1\right)^{\frac{3}{2}}} \\
& +\left(\left(\dot{x}_{i}, \dot{y}_{i}, \dot{z}_{i}\right) \odot\left(\dot{x}_{i}, \dot{y}_{i}, \dot{z}_{i}\right)\right)\left(x_{i}, r Y_{i}, r Z_{i}\right),
\end{aligned}
$$

para $i=1,2$. 
Definimos $f_{i j}=\left(q_{i} \odot q_{j}\right)=\left(x_{i}, y_{i}, z_{i}\right) \odot\left(x_{i}, y_{i}, z_{i}\right)=x_{i} x_{j}+r^{2}\left(Y_{i} Y_{j}-Z_{i} Z_{j}\right)$. Entonces la última ecuación queda

$$
\begin{aligned}
\ddot{q}_{i} & =\sum_{j=1, j \neq i}^{3} \frac{m j\left(\left(x_{j}, r Y_{j}, r Z_{j}\right)+f_{i j}\left(x_{i}, r Y_{i}, r Z_{i}\right)\right)}{\left(f_{i j}^{2}-1\right)^{\frac{3}{2}}}+\left(\left(\dot{x}_{i}, \dot{y}_{i}, \dot{z}_{i}\right) \odot\left(\dot{x}_{i}, \dot{y}_{i}, \dot{z}_{i}\right)\right)\left(x_{i}, r Y_{i}, r Z_{i}\right) \\
& =\sum_{j=1, j \neq i}^{3} \frac{m j\left(\left(x_{j}, r Y_{j}, r Z_{j}\right)+f_{i j}\left(x_{i}, r Y_{i}, r Z_{i}\right)\right)}{\left(f_{i j}^{2}-1\right)^{\frac{3}{2}}}+\left(\dot{q}_{i} \odot \dot{q}_{i}\right)\left(x_{i}, r Y_{i}, r Z_{i}\right), i=1,2 .
\end{aligned}
$$

Como la coordenada $x_{i}$ del cuerpo $i$ se mantiene constante, entonces podemos ver el movimiento de cada particula sobre un plano $y z$, es decir, para el análisis podemos omitir la coordenada $x_{i}$.

$$
\ddot{q}_{i}=\sum_{j \neq i} \frac{m j\left(\left(r Y_{j}, r Z_{j}\right)+f_{i j}\left(r Y_{i}, r Z_{i}\right)\right)}{\left(f_{i j}^{2}-1\right)^{\frac{3}{2}}}+\left(\dot{q}_{i} \odot \dot{q}_{i}\right)\left(r Y_{i}, r Z_{i}\right), i=1,2 .
$$

Ahora, tomando en cuenta que $Q_{i}^{\prime \prime}=r^{2} \ddot{q}_{i}$, tenemos

$$
\begin{aligned}
Q_{i}^{\prime \prime} & =r^{3} \sum_{j \neq i} \frac{m j\left(\left(Y_{j}, Z_{j}\right)+f_{i j}\left(Y_{i}, Z_{i}\right)\right)}{\left(f_{i j}^{2}-1\right)^{\frac{3}{2}}}+r^{3}\left(\dot{q}_{i} \odot \dot{q}_{i}\right)\left(Y_{i}, Z_{i}\right) \\
& =r^{3} \sum_{j \neq i} \frac{m j\left(Q_{j}+f_{i j} Q_{i}\right)}{\left(f_{i j}^{2}-1\right)^{\frac{3}{2}}}+r^{3}\left(\dot{q}_{i} \odot \dot{q}_{i}\right) Q_{i}, i=1,2,3 .
\end{aligned}
$$

Introducimos coordenadas rotatorias $\left(\xi_{i}, \eta_{i}\right), i=1,2$,

$$
\left(\begin{array}{c}
Y_{i} \\
Z_{i}
\end{array}\right)=R(\Omega \tau)\left(\begin{array}{c}
\xi_{i} \\
\eta_{i}
\end{array}\right)
$$

en donde $R(\Omega \tau)$ es la matriz de rotación hiperbólica

$$
R(\Omega \tau)=\left(\begin{array}{cc}
\cosh \Omega \tau & \sinh \Omega \tau \\
\sinh \Omega \tau & \cosh \Omega \tau
\end{array}\right)
$$

Tenemos entonces

$$
\begin{aligned}
& \left(\begin{array}{c}
\xi_{i} \\
\eta_{i}
\end{array}\right)=R^{-1}(\Omega \tau)\left(\begin{array}{c}
Y_{i} \\
Z_{i}
\end{array}\right)=\left(\begin{array}{cc}
\cosh \Omega \tau & -\sinh \Omega \tau \\
-\sinh \Omega \tau & \cosh \Omega \tau
\end{array}\right)\left(\begin{array}{c}
Y_{i} \\
Z_{i}
\end{array}\right) \\
& =\left(\begin{array}{ccc}
\cosh \Omega \tau & Y_{i}-\sinh \Omega \tau & Z_{i} \\
-\sinh \Omega \tau & Y_{i}+\cosh \Omega \tau & Z_{i}
\end{array}\right) .
\end{aligned}
$$

Luego 


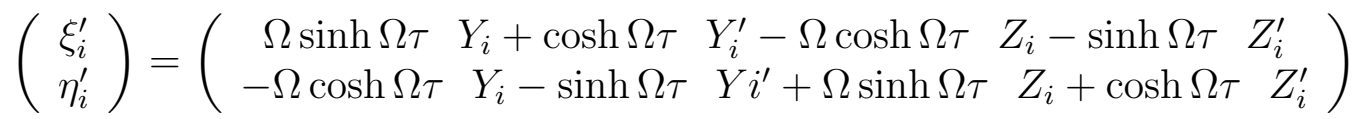

$$
\begin{aligned}
& \left(\begin{array}{c}
\xi_{i}^{\prime \prime} \\
\eta_{i}^{\prime \prime}
\end{array}\right)=\left(\begin{array}{c}
\Omega^{2} \cosh \Omega \tau Y_{i}+2 \Omega \sinh \Omega \tau X_{i}^{\prime}+\cos \Omega \tau Y_{i}^{\prime \prime}-\Omega^{2} \sinh \Omega \tau Z_{i}-2 \Omega \cosh \Omega \tau Z_{i}^{\prime}+ \\
-\sin \Omega \tau Z_{i}^{\prime \prime} \\
-\Omega^{2} \sinh \Omega \tau Y_{i}-2 \Omega \cosh \Omega \tau Y_{i}^{\prime}-\sinh \Omega \tau Y_{i}^{\prime \prime}+\Omega^{2} \cosh \Omega \tau Z_{i}-2 \Omega \sinh \Omega \tau Z_{i}^{\prime}- \\
+\cosh \Omega \tau Z_{i}^{\prime \prime}
\end{array}\right) \\
& =\Omega^{2}\left(\begin{array}{c}
\cosh \Omega \tau Y_{i}-\sinh \Omega \tau Z_{i} \\
-\sinh \Omega \tau Y_{i}+\cos \Omega \tau Z_{i}
\end{array}\right)+2 \Omega\left(\begin{array}{c}
+\sinh \Omega \tau Y_{i}^{\prime}-\cosh \Omega \tau Z_{i}^{\prime} \\
-\cosh \Omega \tau Y_{i}^{\prime}+\sinh \Omega \tau Z_{i}^{\prime}
\end{array}\right) \\
& +\left(\begin{array}{c}
\cosh \Omega \tau Y_{i}^{\prime \prime}-\sinh \Omega \tau Z_{i}^{\prime \prime} \\
-\sinh \Omega \tau Y_{i}^{\prime \prime}+\cosh \Omega \tau Z_{i}^{\prime \prime}
\end{array}\right) \\
& =\Omega^{2}\left(\begin{array}{c}
\cosh \Omega \tau Y_{i}-\sinh \Omega \tau Z_{i} \\
-\sinh \Omega \tau Y_{i}+\cos \Omega \tau Z_{i}
\end{array}\right)+2 \Omega\left(\begin{array}{c}
+\sinh \Omega \tau Y_{i}^{\prime}-\cosh \Omega \tau Z_{i}^{\prime} \\
-\cosh \Omega \tau Y_{i}^{\prime}+\sinh \Omega \tau Z_{i}^{\prime}
\end{array}\right) \\
& +R^{-1}(\Omega \tau)\left(\begin{array}{c}
Y_{i}^{\prime \prime} \\
Z_{i}^{\prime \prime}
\end{array}\right) \text {. }
\end{aligned}
$$

Sumando y restando $2\left(\begin{array}{c}\Omega^{2} \cosh \Omega \tau Y_{i}-\Omega^{2} \sinh \Omega \tau Z_{i} \\ -\Omega^{2} \sinh \Omega \tau Y_{i}+\Omega^{2} \cosh \Omega \tau Z_{i}\end{array}\right)$ obtenemos

$$
\begin{aligned}
\left(\begin{array}{c}
\xi_{i}^{\prime \prime} \\
\eta_{i}^{\prime \prime}
\end{array}\right) & =\Omega^{2}\left(\begin{array}{c}
\cosh \Omega \tau Y_{i}-\sinh \Omega \tau Z_{i} \\
-\sinh \Omega \tau Y_{i}+\cosh \Omega \tau Z_{i}
\end{array}\right)-2 \Omega^{2}\left(\begin{array}{c}
\cosh \Omega \tau Y_{i}-\sinh \Omega \tau Z_{i} \\
-\sinh \Omega \tau Y_{i}+\cosh \Omega \tau Z_{i}
\end{array}\right) \\
& +2 \Omega\left(\begin{array}{c}
\sinh \Omega \tau Y_{i}^{\prime}-\cosh \Omega \tau Z_{i}^{\prime}-\Omega \sinh \Omega \tau Z_{i}+\Omega \cosh \Omega \tau Y_{i} \\
-\cosh \Omega \tau Y_{i}^{\prime}+\sinh \Omega \tau Z_{i}^{\prime}-\Omega \sinh \Omega \tau Y_{i}+\Omega \cosh \Omega \tau Z_{i}
\end{array}\right)+R^{-1}(\Omega \tau)\left(\begin{array}{c}
Y_{i}^{\prime \prime} \\
Z_{i}^{\prime \prime}
\end{array}\right) \\
& =-\Omega^{2}\left(\begin{array}{c}
\xi_{i} \\
\eta_{i}
\end{array}\right)-2 \Omega\left(\begin{array}{c}
\eta_{i}^{\prime} \\
\xi_{i}^{\prime}
\end{array}\right)+R^{-1}(\Omega \tau)\left(\begin{array}{c}
Y_{i}^{\prime \prime} \\
Z_{i}^{\prime \prime}
\end{array}\right) .
\end{aligned}
$$

Desarrollando los valores de $Y^{\prime \prime}$ y $Z^{\prime \prime}$ en

$$
R^{-1}(\Omega \tau)\left(\begin{array}{c}
Y_{i}^{\prime \prime} \\
Z_{i}^{\prime \prime}
\end{array}\right)
$$


y usando (6.88) y (6.89) obtenemos las ecuaciones de movimiento en un sistema rotatorio que gira a velocidad $\Omega$, que quedan como

$$
\begin{aligned}
\left(\begin{array}{c}
\xi_{i}^{\prime \prime} \\
\eta_{i}^{\prime \prime}
\end{array}\right) & =-2 \Omega\left(\begin{array}{c}
\eta_{i}^{\prime} \\
\xi_{i}^{\prime}
\end{array}\right)-\Omega^{2}\left(\begin{array}{c}
\xi_{i} \\
\eta_{i}
\end{array}\right)+r^{2} h_{i}\left(\begin{array}{c}
\xi_{i} \\
\eta_{i}
\end{array}\right) \\
& +\sum_{j=1, j \neq i}^{3} m_{j}\left[\xi_{i}^{2}-\eta_{i}^{2}+\xi_{j}^{2}-\eta_{j}^{2}-2\left(\xi_{i} \xi_{j}-\eta_{i} \eta_{j}\right) \sqrt{\left(r^{2}\left(\eta_{i}^{2}-\xi_{i}^{2}\right)-1\right)\left(r^{2}\left(\eta_{j}^{2}-\xi_{j}^{2}\right)-1\right)}\right. \\
& \left.+r^{2}\left(\left(\xi_{i} \xi_{j}-\eta_{i} \eta_{j}\right)^{2}+\left(\eta_{i}^{2}-\xi_{i}^{2}\right)\left(\eta_{j}^{2}-\xi_{j}^{2}\right)\right)\right]^{-3 / 2}\left[\left(\begin{array}{c}
\xi_{j} \\
\eta_{j}
\end{array}\right)+\left(r^{2}\left(\xi_{i} \xi_{j}-\eta_{i} \eta_{j}\right)\right.\right. \\
& \left.\left.-\sqrt{\left(r^{2}\left(\eta_{i}^{2}-\xi_{i}^{2}\right)-1\right)\left(r^{2}\left(\eta_{j}^{2}-\xi_{j}^{2}\right)-1\right)}\right)\left(\begin{array}{c}
\xi_{i} \\
\eta_{i}
\end{array}\right)\right]
\end{aligned}
$$

donde

$$
h_{i}=\Omega^{2}\left(\eta_{i}^{2}-\xi_{i}^{2}\right)+2 \Omega\left(\eta_{i} \xi_{i}^{\prime}-\xi_{i} \eta_{i}^{\prime}\right)+\left(\left(\xi_{i}^{\prime 2}\right)-\left(\eta_{i}^{\prime 2}\right)\right)+\frac{r^{2}}{r^{2}\left(\eta_{i}^{2}-\xi_{i}^{2}\right)-1}\left(\eta_{i} \eta_{i}^{\prime}-\xi_{i} \xi_{i}^{\prime}\right)^{2}
$$

para $i=1,2$.

Podemos ver que un punto fijo del sistema es $\xi_{1}=0, \eta_{1}=1, \xi_{2}=0, \eta_{2}=1, \xi_{1}^{\prime}=0, \eta_{1}^{\prime},=$ $0, \xi_{2}^{\prime}=0, \eta_{2}^{\prime}=0$.

La matriz $D f$ correspodiente a los equilibrios relativos con estos valores de masas es una matriz de tamaño $8 \times 8$ de la forma

$$
D f=\left(\begin{array}{cc}
0 & I \\
A & B
\end{array}\right)
$$

en donde $I$ es la matriz identidad de tamaño $4 \times 4$. La matriz $A$ en este caso tiene la forma

$$
A=\left(\begin{array}{llll}
a & 0 & b & 0 \\
0 & c & 0 & d \\
b & 0 & a & 0 \\
0 & d & 0 & c
\end{array}\right)
$$

donde 


$$
\begin{aligned}
& a=\Omega^{2} r^{2}-\Omega^{2}-\frac{2 r^{2}-1}{\left(4 r^{2}-4\right)^{3 / 2}}, \\
& b=\frac{1}{\left(4 r^{2}-4\right)^{3 / 2}}, \\
& c=3 \Omega^{2} r^{2}-\Omega^{2}+\frac{3\left(2 r^{2}-2\right)\left(4 r^{2}-2\right)}{\left(4 r^{2}-4\right)^{5 / 2}}-\frac{4 r^{2}-1}{\left(4 r^{2}-4\right)^{3 / 2}}, \\
& d=\frac{3\left(2 r^{2}-2\right)\left(4 r^{2}-2\right)}{\left(4 r^{2}-4\right)^{5 / 2}}-\frac{2 r^{2}-1}{\left(4 r^{2}-4\right)^{3 / 2}} .
\end{aligned}
$$

La matriz $B$

$$
B=\left(\begin{array}{rrrr}
0 & -2 \Omega & 0 & 0 \\
2 \Omega r^{2}-2 \Omega & 0 & 0 & 0 \\
0 & 0 & 0 & -2 \Omega \\
0 & 0 & 2 \Omega r^{2}-2 \Omega & 0
\end{array}\right) .
$$

Recordemos que la velocidad angular debe satisfacer

$$
\Omega^{2}=\frac{1}{4\left(r^{2}-1\right)^{3 / 2}} .
$$

Usando (6.101) en las entradas de la matriz $A$ podemos reescribir

$$
\begin{aligned}
& a=-\frac{\Omega^{2}}{2}, \\
& b=\frac{\Omega^{2}}{2}, \\
& c=\Omega^{2}\left(-2+4 r^{2}\right), \\
& d=\Omega^{2}\left(2 r^{2}-1\right) .
\end{aligned}
$$

Notemos que también a la matriz $D f$ la podemos escribir de la siguiente manera

$$
D f=\left(\begin{array}{cc}
0 & I \\
\Omega^{2} \widehat{A} & \Omega \widehat{B}
\end{array}\right) .
$$


Los eigenvalores de la matriz $D f$ son los ceros de la ecuación $\operatorname{det}(D f-\lambda I)=0$. Definamos $\mu$ de tal manera que $\lambda=\Omega \mu$, y así buscaremos los ceros de la ecuación $\operatorname{det}(D f-\Omega \mu I)=0$. Como vimos en la sección 5.2.1 los eigenvalores son los ceros de la ecuación $D=0$, donde $D(\mu)=\widehat{A}+\mu \widehat{B}-\mu^{2} I$.

Si denotamos a $r^{2}$ por $R$ tenemos

$$
\operatorname{det}(D)=p(\mu)=\mu^{8}-4 \mu^{4} R^{2}-3 \mu^{6}+4 \mu^{2} R^{2}+3 \mu^{4}-\mu^{2},
$$

que podemos reescribir como sigue

$$
p(\mu)=(\mu-1)(\mu+1)\left(\mu^{2}-2 R-1\right)\left(\mu^{2}+2 R-1\right) \mu^{2} .
$$

De la última ecuación podemos ver que la matriz $D f$ tiene eigenvalores reales.

\subsubsection{Problema de Tres Cuerpos}

En el problema de los tres cuerpos se tienen resultados sobre el problema restringido, es decir en el caso en donde la partícula que está movéndose sobre la hipérbola que pasa por $(0,0,1)$ es de masa despreciable y las otras dos se encuentran girando sobre hipérbolas a una distancia $x$, ver figura (6.7). A continuación mostraremos nuestro resultado

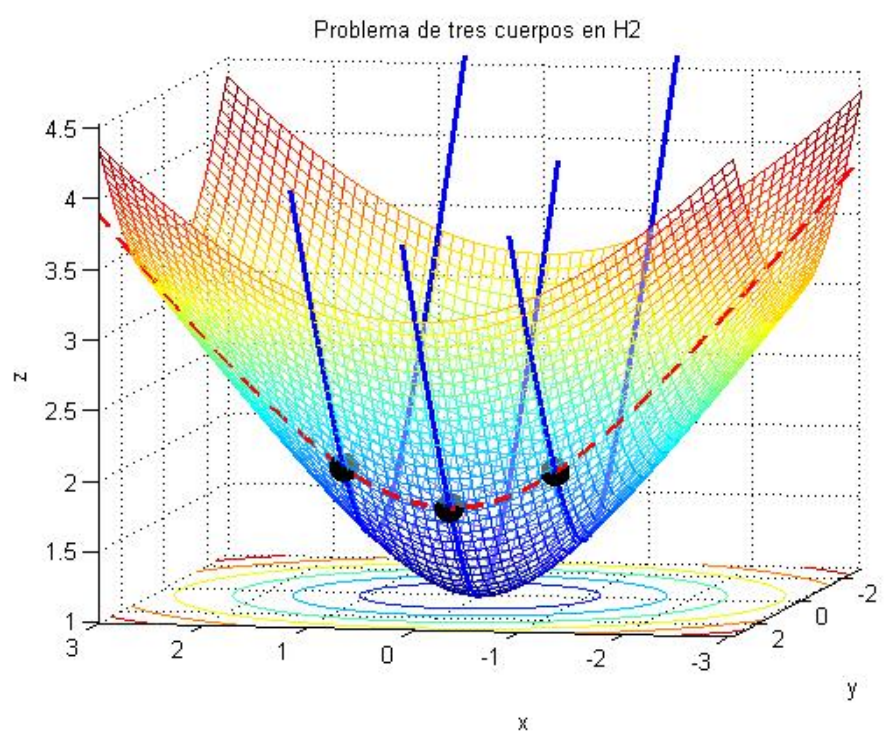

Figura 6.7: Equilibrios relativos hiperbólicos del problema de tres cuerpos en $H^{2}$. Uno de ellos se encuentra moviéndose sobre la hipérbola que pasa por $(0,0,1)$ con $x=0$ y los otros dos se encuentran moviéndose sobre hipérbolas con $x= \pm$ cte (curvas continuas-azules), los tres cuerpos mantienen la misma distancia, en todo tiempo, medida sobre geodésicas (curva punteada-roja). 


\subsubsection{Problema Restringido}

Los resultados de estabilidad de equilibrios relativos hiperbolicos de tipo eulerianos los mencionamos a través del siguiente

Teorema 6.2.2 Consideremos órbitas eulerianas del problema curvado de los tres cuerpos en $H^{2}$, donde el cuerpo $m_{i}$ tiene coordenadas $q_{i}=\left(x_{i}, y_{i}, z_{i}\right)$. Si las masas $m_{1}$ y $m_{2}$ se mueven a lo largo de hipérbolas de la forma $x_{1}=$ cte, $x_{2}=-x_{1}, y_{1}=y_{2}=r \sinh (t), z_{1}=z_{2}=$ $r \cosh (t), t \in \mathbb{R}$, y la partícula de $m_{3}$ de masa despreciable se encuentra moviéndose sobre la hipérbola que pasa por $(0,0,1)$, entonces las órbitas generadas son inestables para cualquier $r>1$.

\section{Demostración}

Para estudiar la estabilidad de equilibrios relativos hiperbólicos introduciremos coordenadas rotatorias, y consideramos una partícula de masa $m_{3}=m$ que se encuentra sobre la hipérbola que pasa por $(0,0,1)$ que después haremos tender a cero. Tomemos en cuenta valores de masas $m_{1}$ y $m_{2}$ normalizadas. Además consideremos los siguientes cambios de coordenadas y reescalamiento de tiempo, este último para simplificar el valor de $\omega$.

$$
\begin{aligned}
\Omega^{2} & =\frac{\omega^{2} t^{2}}{\tau^{2}} \\
& =\frac{\omega^{2} r^{3} \tau^{2}}{\tau^{2}} \\
& =\omega^{2} r^{3} \\
& =\left(\frac{m_{1}}{4 x^{2}|x|\left(x^{2}+1\right)^{\frac{3}{2}}}+\frac{m_{3}}{x^{2}|x|\left(x^{2}+1\right)^{\frac{1}{2}}}\right) r^{3} \\
& =\frac{m_{1}+4 r^{2} m_{3}}{4\left(r^{2}-1\right)^{\frac{3}{2}}} \\
& =\frac{1+4 r^{2} m}{4\left(r^{2}-1\right)^{\frac{3}{2}}} .
\end{aligned}
$$

Introducimos la notación 


$$
\begin{aligned}
Y_{i}^{\prime} & =r^{\frac{1}{2}} \dot{y}_{i}, \\
Y_{i}^{\prime \prime} & =r^{2} \ddot{y}_{i}, \\
Z_{i}^{\prime} & =r^{\frac{1}{2}} \dot{z}_{i}, \\
Z_{i}^{\prime \prime} & =r^{2} \ddot{z}_{i},
\end{aligned}
$$

para $i=1,2,3$.

Ahora aplicamos las coordenadas reescaladas a las ecuaciones de movimiento, teniendo

$$
\begin{aligned}
\ddot{q}_{i} & =\sum_{j=1, j \neq i}^{3} \frac{m_{j}\left(q_{j}+\left(q_{i} \odot q_{j}\right) q_{i}\right)}{\left(\left(q_{i} \odot q_{j}\right)^{2}-1\right)^{\frac{3}{2}}}+\left(\dot{q}_{i} \odot \dot{q}_{i}\right) q_{i} \\
& =\sum_{j=1, j \neq i}^{3} \frac{m_{j}\left(\left(x_{j}, y_{j}, z_{j}\right)+\left(\left(x_{i}, y_{i}, z_{i}\right) \odot\left(x_{j}, y_{j}, z_{j}\right)\right)\left(x_{i}, y_{i}, z_{i}\right)\right)}{\left(\left(\left(x_{i}, y_{i}, z_{i}\right) \odot\left(x_{j}, y_{j}, z_{j}\right)\right)^{2}-1\right)^{\frac{3}{2}}} \\
& +\left(\left(\dot{x}_{i}, \dot{y}_{i}, \dot{z}_{i}\right) \odot\left(\dot{x}_{i}, \dot{y}_{i}, \dot{z}_{i}\right)\right)\left(x_{i}, y_{i}, z_{i}\right) \\
& =\sum_{j=1, j \neq i}^{3} \frac{m_{j}\left(\left(x_{j}, r Y_{j}, r Z_{j}\right)+\left(\left(x_{i}, y_{i}, z_{i}\right) \odot\left(x_{j}, y_{j}, z_{j}\right)\right)\left(x_{i}, r Y_{i}, r Z_{i}\right)\right)}{\left(\left(\left(x_{i}, y_{i}, z_{i}\right) \odot\left(x_{i}, y_{i}, z_{i}\right)\right)^{2}-1\right)^{\frac{3}{2}}} \\
& +\left(\left(\dot{x}_{i}, \dot{y}_{i}, \dot{z}_{i}\right) \odot\left(\dot{x}_{i}, \dot{y}_{i}, \dot{z}_{i}\right)\right)\left(x_{i}, r Y_{i}, r Z_{i}\right),
\end{aligned}
$$

para $i=1,2,3$.

Definimos $f_{i j}=\left(q_{i} \odot q_{j}\right)=\left(x_{i}, y_{i}, z_{i}\right) \odot\left(x_{i}, y_{i}, z_{i}\right)=x_{i} x_{j}+r^{2}\left(Y_{i} Y_{j}-Z_{i} Z_{j}\right)$. Entonces la última ecuación queda

$$
\begin{aligned}
\ddot{q}_{i} & =\sum_{j=1, j \neq i}^{3} \frac{m j\left(\left(x_{j}, r Y_{j}, r Z_{j}\right)+f_{i j}\left(x_{i}, r Y_{i}, r Z_{i}\right)\right)}{\left(f_{i j}^{2}-1\right)^{\frac{3}{2}}}+\left(\left(\dot{x}_{i}, \dot{y}_{i}, \dot{z}_{i}\right) \odot\left(\dot{x}_{i}, \dot{y}_{i}, \dot{z}_{i}\right)\right)\left(x_{i}, r Y_{i}, r Z_{i}\right) \\
& =\sum_{j=1, j \neq i}^{3} \frac{m j\left(\left(x_{j}, r Y_{j}, r Z_{j}\right)+f_{i j}\left(x_{i}, r Y_{i}, r Z_{i}\right)\right)}{\left(f_{i j}^{2}-1\right)^{\frac{3}{2}}}+\left(\dot{q}_{i} \odot \dot{q}_{i}\right)\left(x_{i}, r Y_{i}, r Z_{i}\right), \quad i=1,2,3 .
\end{aligned}
$$

Como la coordenada $x_{i}$ del cuerpo $i$ se mantiene constante, entonces podemos ver el movimiento de cada particula sobre un plano $y z$, es decir, para el análisis podemos omitir la coordenada $x_{i}$.

$$
\ddot{q}_{i}=\sum_{j \neq i} \frac{m j\left(\left(r Y_{j}, r Z_{j}\right)+f_{i j}\left(r Y_{i}, r Z_{i}\right)\right)}{\left(f_{i j}^{2}-1\right)^{\frac{3}{2}}}+\left(\dot{q}_{i} \odot \dot{q}_{i}\right)\left(r Y_{i}, r Z_{i}\right), i=1,2,3 .
$$


Ahora, tomando en cuenta que $Q_{i}^{\prime \prime}=r^{2} \ddot{q}_{i}$, tenemos

$$
\begin{aligned}
Q_{i}^{\prime \prime} & =r^{3} \sum_{j \neq i} \frac{m j\left(\left(Y_{j}, Z_{j}\right)+f_{i j}\left(Y_{i}, Z_{i}\right)\right)}{\left(f_{i j}^{2}-1\right)^{\frac{3}{2}}}+r^{3}\left(\dot{q}_{i} \odot \dot{q}_{i}\right)\left(Y_{i}, Z_{i}\right) \\
& =r^{3} \sum_{j \neq i} \frac{m j\left(Q_{j}+f_{i j} Q_{i}\right)}{\left(f_{i j}^{2}-1\right)^{\frac{3}{2}}}+r^{3}\left(\dot{q}_{i} \odot \dot{q}_{i}\right) Q_{i}, \quad i=1,2,3 .
\end{aligned}
$$

Introducimos coordenadas rotatorias $\left(\xi_{i}, \eta_{i}\right), i=1,2,3$,

$$
\left(\begin{array}{c}
Y_{i} \\
Z_{i}
\end{array}\right)=R(\Omega \tau)\left(\begin{array}{c}
\xi_{i} \\
\eta_{i}
\end{array}\right)
$$

en donde $R(\Omega \tau)$ es la matriz de rotación hiperbólica

$$
R(\Omega \tau)=\left(\begin{array}{cc}
\cosh \Omega \tau & \sinh \Omega \tau \\
\sinh \Omega \tau & \cosh \Omega \tau
\end{array}\right)
$$

Tenemos entonces

$$
\begin{aligned}
& \left(\begin{array}{c}
\xi_{i} \\
\eta_{i}
\end{array}\right)=R^{-1}(\Omega \tau)\left(\begin{array}{c}
Y_{i} \\
Z_{i}
\end{array}\right)=\left(\begin{array}{cc}
\cosh \Omega \tau & -\sinh \Omega \tau \\
-\sinh \Omega \tau & \cosh \Omega \tau
\end{array}\right)\left(\begin{array}{c}
Y_{i} \\
Z_{i}
\end{array}\right) \\
& =\left(\begin{array}{ccc}
\cosh \Omega \tau & Y_{i}-\sinh \Omega \tau & Z_{i} \\
-\sinh \Omega \tau & Y_{i}+\cosh \Omega \tau & Z_{i}
\end{array}\right) .
\end{aligned}
$$

Luego

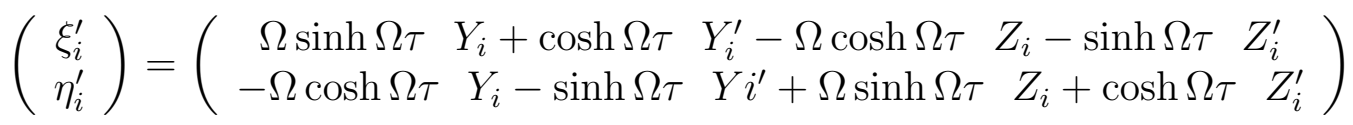




$$
\begin{aligned}
\left(\begin{array}{c}
\xi_{i}^{\prime \prime} \\
\eta_{i}^{\prime \prime}
\end{array}\right)= & \left(\begin{array}{c}
\Omega^{2} \cosh \Omega \tau Y_{i}+2 \Omega \sinh \Omega \tau X_{i}^{\prime}+\cos \Omega \tau Y_{i}^{\prime \prime}-\Omega^{2} \sinh \Omega \tau Z_{i}-2 \Omega \cosh \Omega \tau Z_{i}^{\prime}+ \\
-\sin \Omega \tau Z_{i}^{\prime \prime} \\
-\Omega^{2} \sinh \Omega \tau Y_{i}-2 \Omega \cosh \Omega \tau Y_{i}^{\prime}-\sinh \Omega \tau Y_{i}^{\prime \prime}+\Omega^{2} \cosh \Omega \tau Z_{i}-2 \Omega \sinh \Omega \tau Z_{i}^{\prime}- \\
+\cosh \Omega \tau Z_{i}^{\prime \prime}
\end{array}\right) \\
= & \Omega^{2}\left(\begin{array}{c}
\cosh \Omega \tau Y_{i}-\sinh \Omega \tau Z_{i} \\
-\sinh \Omega \tau Y_{i}+\cos \Omega \tau Z_{i}
\end{array}\right)+2 \Omega\left(\begin{array}{c}
+\sinh \Omega \tau Y_{i}^{\prime}-\cosh \Omega \tau Z_{i}^{\prime} \\
-\cosh \Omega \tau Y_{i}^{\prime}+\sinh \Omega \tau Z_{i}^{\prime}
\end{array}\right) \\
& +\left(\begin{array}{c}
\cosh \Omega \tau Y_{i}^{\prime \prime}-\sinh \Omega \tau Z_{i}^{\prime \prime} \\
-\sinh \Omega \tau Y_{i}^{\prime \prime}+\cosh \Omega \tau Z_{i}^{\prime \prime}
\end{array}\right) \\
& =\Omega^{2}\left(\begin{array}{c}
\cosh \Omega \tau Y_{i}-\sinh \Omega \tau Z_{i} \\
-\sinh \Omega \tau Y_{i}+\cos \Omega \tau Z_{i}
\end{array}\right)+2 \Omega\left(\begin{array}{c}
+\sinh \Omega \tau Y_{i}^{\prime}-\cosh \Omega \tau Z_{i}^{\prime} \\
-\cosh \Omega \tau Y_{i}^{\prime}+\sinh \Omega \tau Z_{i}^{\prime}
\end{array}\right) \\
& +R^{-1}(\Omega \tau)\left(\begin{array}{c}
Y_{i}^{\prime \prime} \\
Z_{i}^{\prime \prime}
\end{array}\right) .
\end{aligned}
$$

Sumando y restando $2\left(\begin{array}{c}\Omega^{2} \cosh \Omega \tau Y_{i}-\Omega^{2} \sinh \Omega \tau Z_{i} \\ -\Omega^{2} \sinh \Omega \tau Y_{i}+\Omega^{2} \cosh \Omega \tau Z_{i}\end{array}\right)$ obtenemos

$$
\begin{aligned}
\left(\begin{array}{c}
\xi_{i}^{\prime \prime} \\
\eta_{i}^{\prime \prime}
\end{array}\right) & =\Omega^{2}\left(\begin{array}{c}
\cosh \Omega \tau Y_{i}-\sinh \Omega \tau Z_{i} \\
-\sinh \Omega \tau Y_{i}+\cosh \Omega \tau Z_{i}
\end{array}\right)-2 \Omega^{2}\left(\begin{array}{c}
\cosh \Omega \tau Y_{i}-\sinh \Omega \tau Z_{i} \\
-\sinh \Omega \tau Y_{i}+\cosh \Omega \tau Z_{i}
\end{array}\right) \\
& +2 \Omega\left(\begin{array}{c}
\sinh \Omega \tau Y_{i}^{\prime}-\cosh \Omega \tau Z_{i}^{\prime}-\Omega \sinh \Omega \tau Z_{i}+\Omega \cosh \Omega \tau Y_{i} \\
-\cosh \Omega \tau Y_{i}^{\prime}+\sinh \Omega \tau Z_{i}^{\prime}-\Omega \sinh \Omega \tau Y_{i}+\Omega \cosh \Omega \tau Z_{i}
\end{array}\right) \\
& +R^{-1}(\Omega \tau)\left(\begin{array}{c}
Y_{i}^{\prime \prime} \\
Z_{i}^{\prime \prime}
\end{array}\right) \\
& =-\Omega^{2}\left(\begin{array}{c}
\xi_{i} \\
\eta_{i}
\end{array}\right)-2 \Omega\left(\begin{array}{c}
\eta_{i}^{\prime} \\
\xi_{i}^{\prime}
\end{array}\right)+R^{-1}(\Omega \tau)\left(\begin{array}{c}
Y_{i}^{\prime \prime} \\
Z_{i}^{\prime \prime}
\end{array}\right) .
\end{aligned}
$$

Desarrollando los valores de $Y^{\prime \prime}$ y $Z^{\prime \prime}$ en

$$
R^{-1}(\Omega \tau)\left(\begin{array}{c}
Y_{i}^{\prime \prime} \\
Z_{i}^{\prime \prime}
\end{array}\right)
$$

y usando (6.111) y (6.112) obtenemos las ecuaciones de movimiento en un sistema rotatorio que gira a velocidad $\Omega$, que quedan como 


$$
\begin{aligned}
\left(\begin{array}{c}
\xi_{i}^{\prime \prime} \\
\eta_{i}^{\prime \prime}
\end{array}\right) & =-2 \Omega\left(\begin{array}{c}
\eta_{i}^{\prime} \\
\xi_{i}^{\prime}
\end{array}\right)-\Omega^{2}\left(\begin{array}{c}
\xi_{i} \\
\eta_{i}
\end{array}\right)+r^{2} h_{i}\left(\begin{array}{c}
\xi_{i} \\
\eta_{i}
\end{array}\right) \\
& +\sum_{j=1, j \neq i}^{3} m_{j}\left[\xi_{i}^{2}-\eta_{i}^{2}+\xi_{j}^{2}-\eta_{j}^{2}-2\left(\xi_{i} \xi_{j}-\eta_{i} \eta_{j}\right) \sqrt{\left(r^{2}\left(\eta_{i}^{2}-\xi_{i}^{2}\right)-1\right)\left(r^{2}\left(\eta_{j}^{2}-\xi_{j}^{2}\right)-1\right)}\right. \\
& \left.+r^{2}\left(\left(\xi_{i} \xi_{j}-\eta_{i} \eta_{j}\right)^{2}+\left(\eta_{i}^{2}-\xi_{i}^{2}\right)\left(\eta_{j}^{2}-\xi_{j}^{2}\right)\right)\right]^{-3 / 2}\left[\left(\begin{array}{c}
\xi_{j} \\
\eta_{j}
\end{array}\right)+\left(r^{2}\left(\xi_{i} \xi_{j}-\eta_{i} \eta_{j}\right)\right.\right. \\
& \left.\left.-\sqrt{\left(r^{2}\left(\eta_{i}^{2}-\xi_{i}^{2}\right)-1\right)\left(r^{2}\left(\eta_{j}^{2}-\xi_{j}^{2}\right)-1\right)}\right)\left(\begin{array}{c}
\xi_{i} \\
\eta_{i}
\end{array}\right)\right]
\end{aligned}
$$

donde

$$
h_{i}=\Omega^{2}\left(\eta_{i}^{2}-\xi_{i}^{2}\right)+2 \Omega\left(\eta_{i} \xi_{i}^{\prime}-\xi_{i} \eta_{i}^{\prime}\right)+\left(\left(\xi_{i}^{\prime 2}\right)-\left(\eta_{i}^{\prime 2}\right)\right)+\frac{r^{2}}{r^{2}\left(\eta_{i}^{2}-\xi_{i}^{2}\right)-1}\left(\eta_{i} \eta_{i}^{\prime}-\xi_{i} \xi_{i}^{\prime}\right)^{2},
$$

para $i=1,2,3$.

Podemos ver que un punto fijo del sistema es $\xi_{1}=0, \eta_{1}=1, \xi_{2}=0, \eta_{2}=1, \xi_{3}=0, \eta_{3}=$ $1, \xi_{1}^{\prime}=0, \eta_{1}^{\prime},=0, \xi_{2}^{\prime}=0, \eta_{2}^{\prime}=0, \xi_{3}^{\prime}=0, \eta_{3}^{\prime}=0$.

Estudiaremos los eigenvalores del campo vectorial de la diferencial de la función de la parte derecha de (6.119), escrito como un sistema de 12 ecuaciones evaluado en el punto fijo que acabamos de mostrar.

La estructura de dicho campo vectorial tiene la forma de la siguiente matriz cuadrada

$$
D f=\left(\begin{array}{cc}
0 & I \\
A & B
\end{array}\right)
$$

donde $I$ es la matriz identidad de tamaño $6 \times 6$, y $A$ y $B$ están dadas de la siguiente forma

$$
B=\left(\begin{array}{cccccc}
0 & -2 \Omega & 0 & 0 & 0 & 0 \\
2 \Omega r^{2}-2 \Omega & 0 & 0 & 0 & 0 & 0 \\
0 & 0 & 0 & -2 \Omega & 0 & 0 \\
0 & 0 & 2 \Omega r^{2}-2 \Omega & 0 & 0 & 0 \\
0 & 0 & 0 & 0 & 0 & -2 \Omega \\
0 & 0 & 0 & 0 & 2 \Omega r^{2}-2 \Omega & 0
\end{array}\right)
$$




$$
A=\left(\begin{array}{cccccc}
a & 0 & b & 0 & e * m & 0 \\
0 & c & 0 & d & 0 & f * m \\
b & 0 & a & 0 & e * m & 0 \\
0 & k & 0 & v & 0 & f * m \\
g & 0 & g & 0 & h & 0 \\
0 & i & 0 & i & 0 & j
\end{array}\right),
$$

donde $a, b, c, d, e, f, g, h$ están dados por los siguientes valores

$$
\begin{aligned}
& a=\frac{r^{2}\left(4 r^{2} m+1\right)}{4\left(r^{2}-1\right)^{\frac{3}{2}}}-\frac{4 r^{2} m+1}{4\left(r^{2}-1\right)^{\frac{3}{2}}}-\frac{2 r^{2}-1}{8\left(r^{2}-1\right)^{\frac{3}{2}}}-\frac{\sqrt{2} r^{2} m}{4\left(r^{2}-1\right)^{\frac{3}{2}}}, \\
& b=\frac{1}{8\left(r^{2}-1\right)^{\frac{3}{2}}}, \\
& c=\frac{6 r^{2}-3}{8\left(r^{2}-1\right)^{\frac{3}{2}}}-\frac{4 r^{2}-1}{8\left(r^{2}-1\right)^{\frac{3}{2}}}-\frac{4 r^{2} m+1}{4\left(r^{2}-1\right)^{\frac{3}{2}}}+\frac{3 r^{2}\left(4 r^{2} m+1\right)}{4\left(r^{2}-1\right)^{\frac{3}{2}}}+\frac{3 \sqrt{2} m\left(2 r^{2}-1\right)}{8\left(r^{2}-1\right)^{\frac{3}{2}}}-\frac{\sqrt{2} r^{2} m}{2\left(r^{2}-1\right)^{\frac{3}{2}}}, \\
& d=\frac{6 r^{2}-3}{8\left(r^{2}-1\right)^{\frac{3}{2}}}-\frac{2 r^{2}-1}{8\left(r^{2}-1\right)^{\frac{3}{2}}},
\end{aligned}
$$




$$
\begin{aligned}
& e=\frac{\sqrt{2}}{4\left(r^{2}-1\right)^{\frac{3}{2}}}, \\
& f=\frac{\sqrt{2}\left(6 r^{2}-3\right)}{8\left(r^{2}-1\right)^{\frac{3}{2}}}-\frac{\sqrt{2}}{4 \sqrt{r^{2}-1}},
\end{aligned}
$$$$
g=\frac{\sqrt{2}}{4\left(r^{2}-1\right)^{\frac{3}{2}}}
$$$$
h=\frac{r^{2}\left(4 r^{2}+1\right)}{4\left(r^{2}-1\right)^{\frac{3}{2}}}-\frac{\sqrt{2} r^{2}}{2\left(r^{2}-1\right)^{\frac{3}{2}}}-\frac{4 r^{2}+1}{4\left(r^{2}-1\right)^{\frac{3}{2}}},
$$$$
i=\frac{\sqrt{2}\left(6 r^{2}-3\right)}{8\left(r^{2}-1\right)^{\frac{3}{2}}}-\frac{\sqrt{2}}{4 \sqrt{r^{2}-1}}
$$$$
j=\frac{3 r^{2}\left(4 r^{2}+1\right)}{4\left(r^{2}-1\right)^{\frac{3}{2}}}-\frac{\sqrt{2} r^{2}}{\left(r^{2}-1\right)^{\frac{3}{2}}}-\frac{4 r^{2}+1}{4\left(r^{2}-1\right)^{\frac{3}{2}}}+\frac{\sqrt{2}\left(6 r^{2}-3\right)}{4\left(r^{2}-1\right)^{\frac{3}{2}}},
$$

$$
\begin{aligned}
& k=\frac{6 r^{2}-3}{8\left(r^{2}-1\right)^{\frac{3}{2}}}-\frac{2 r^{2}-1}{8\left(r^{2}-1\right)^{\frac{3}{2}}}-\frac{\sqrt{2} r^{2} m}{4\left(r^{2}-1\right)^{\frac{3}{2}}}, \\
& v=\frac{6 r^{2}-3}{8\left(r^{2}-1\right)^{\frac{3}{2}}}-\frac{4 r^{2}-1}{8\left(r^{2}-1\right)^{\frac{3}{2}}}-\frac{4 r^{2} m+1}{4\left(r^{2}-1\right)^{\frac{3}{2}}}+\frac{3 r^{2}\left(4 r^{2} m+1\right)}{4\left(r^{2}-1\right)^{\frac{3}{2}}}+\frac{3 \sqrt{2} m\left(2 r^{2}-1\right)}{8\left(r^{2}-1\right)^{\frac{3}{2}}}-\frac{\sqrt{2} r^{2} m}{4\left(r^{2}-1\right)^{\frac{3}{2}}} .
\end{aligned}
$$

A la matriz $D f$ la podemos reescribir como

$$
D f=\left(\begin{array}{cc}
0 & I \\
A & \Omega \widetilde{B}
\end{array}\right),
$$

con

$$
\widetilde{B}=\left(\begin{array}{cccccc}
0 & -2 & 0 & 0 & 0 & 0 \\
2 r^{2}-2 & 0 & 0 & 0 & 0 & 0 \\
0 & 0 & 0 & -2 & 0 & 0 \\
0 & 0 & 2 r^{2}-2 & 0 & 0 & 0 \\
0 & 0 & 0 & 0 & 0 & -2 \\
0 & 0 & 0 & 0 & 2 r^{2}-2 & 0
\end{array}\right)
$$


Los vectores propios de $D f$ son los ceros de la ecuación $\operatorname{det}(D f-\lambda I)=0$. Definamos $\mu$ tal que $\lambda=\Omega \mu$, por lo que buscaremos los ceros de la ecuación $\operatorname{det}(D f-\Omega \mu I)=0$. La condicón que buscaremos para los valores propios puede ser escrita como

$$
\left(\begin{array}{cc}
0 & I \\
A & \Omega \widetilde{B}
\end{array}\right)\left(\begin{array}{l}
u \\
v
\end{array}\right)=\Omega \mu\left(\begin{array}{l}
u \\
v
\end{array}\right),
$$

donde $u, v$ están formados por las primeras y últimas cuatro componentes, respectivamente, del eigenvector correspondiente al eigenvalor $\Omega \mu$.

Así la condición del eigenvalor con parámetro $\mu$ se sigue de

$$
v=\Omega \mu u, \quad A u+\Omega \widetilde{B} \Omega \mu u=\Omega \mu v=\Omega^{2} \mu^{2} u .
$$

Denotemos al polinomio caracteristico como $p(\mu)$. La condición de valor propio debe satisfacer

$$
p(\mu)=\operatorname{det}\left(A+\Omega^{2} \mu \widetilde{B}-\Omega^{2} \mu^{2}\right)=0 .
$$

Si denotamos a $r^{2}$ por $R$ entonces 
$p(\mu)=-\frac{1}{8192(R-1)^{9}}\left(24 m-228 R m+72 \sqrt{2} m+16 \mu^{2} R-48 \mu^{4} R+48 \mu^{6} R-16 \mu^{8} R\right.$

$+6 \sqrt{2} \mu^{2}-12 \sqrt{2} \mu^{4}+12 \sqrt{2} \mu^{8}-6 \sqrt{2} \mu^{10}-46 \mu^{2} m+66 \mu^{4} m-18 \mu^{6} m-26 \mu^{8} m$

$-864 R m^{2}+492 R^{2} m+1496 R m^{3}+360 R^{3} m+480 R m^{4}-2088 R^{4} m+1920 R^{5} m$

$-480 R^{6} m+48 \sqrt{2} m^{2}+96 \sqrt{2} m^{3}+2 \mu^{2}-10 \mu^{4}+20 \mu^{6}-20 \mu^{8}+10 \mu^{10}-2 \mu^{12}$

$-18 \mu^{2} R^{2}-64 \mu^{2} R^{3}+40 \mu^{2} R^{4}+54 \mu^{4} R^{2}+64 \mu^{4} R^{3}-40 \mu^{4} R^{4}-54 \mu^{6} R^{2}+18 \mu^{8} R^{2}$

$+280 m^{2}+36 m^{3}+151 \mu^{2} m^{2}+72 \mu^{2} m^{3}+145 \mu^{4} m^{2}+36 \mu^{4} m^{3}+9 \mu^{6} m^{2}-9 \mu^{8} m^{2}$

$-1880 R^{2} m^{2}-8980 R^{2} m^{3}+10648 R^{3} m^{2}-210 R^{2} m^{4}+4284 R^{3} m^{3}-9808 R^{4} m^{2}+576 R^{2} m^{5}$

$-18068 R^{3} m^{4}+62324 R^{4} m^{3}-12280 R^{5} m^{2}-6096 R^{3} m^{5}+43882 R^{4} m^{4}-150632 R^{5} m^{3}$

$+23888 R^{6} m^{2}+5664 R^{4} m^{5}+106556 R^{5} m^{4}+116624 R^{6} m^{3}-11904 R^{7} m^{2}-2592 R^{4} m^{6}$

$+45072 R^{5} m^{5}-570816 R^{6} m^{4}-6592 R^{7} m^{3}+1920 R^{8} m^{2}+14016 R^{5} m^{6}+31840 R^{6} m^{5}$

$+921184 R^{7} m^{4}-24704 R^{8} m^{3}-14688 R^{6} m^{6}-744192 R^{7} m^{5}-733376 R^{8} m^{4}+6144 R^{9} m^{3}$

$+17600 R^{7} m^{6}+1823744 R^{8} m^{5}+319488 R^{9} m^{4}-299520 R^{8} m^{6}-1998336 R^{9} m^{5}-69120 R^{10} m^{4}$

$+1000960 R^{9} m^{6}+1099776 R^{10} m^{5}-1379328 R^{10} m^{6}-258048 R^{11} m^{5}+884736 R^{11} m^{6}$

$-221184 R^{12} m^{6}-32 \sqrt{2} \mu^{2} R^{2}+24 \sqrt{2} \mu^{2} R^{3}+32 \sqrt{2} \mu^{2} R^{4}+24 \sqrt{2} \mu^{4} R^{2}$

$-24 \sqrt{2} \mu^{4} R^{3}-32 \sqrt{2} \mu^{4} R^{4}+8 \sqrt{2} \mu^{8} R^{2}-1598 \mu^{2} R m^{2}-840 \mu^{2} R^{2} m+248 \mu^{2} R m^{3}$

$-1408 \mu^{2} R^{3} m+576 \mu^{2} R m^{4}+2544 \mu^{2} R^{4} m+882 \mu^{4} R m^{2}+128 \mu^{4} R^{2} m-1472 \mu^{2} R^{5} m$

$+1148 \mu^{4} R m^{3}+1152 \mu^{4} R^{3} m+320 \mu^{2} R^{6} m+288 \mu^{4} R m^{4}-792 \mu^{4} R^{4} m-326 \mu^{6} R m^{2}$

$+92 \mu^{6} R^{2} m+192 \mu^{4} R^{5} m+108 \mu^{6} R m^{3}-424 \mu^{6} R^{3} m-64 \mu^{6} R^{4} m-422 \mu^{8} R m^{2}$

$-40 \mu^{8} R^{2} m-144 \mu^{8} R m^{3}+176 \mu^{8} R^{3} m+96 \sqrt{2} \mu^{2} m^{3}-48 \sqrt{2} \mu^{4} m^{2}-4422 \sqrt{2} R^{2} m^{2}$

$-3610 \sqrt{2} R^{2} m^{3}+14836 \sqrt{2} R^{3} m^{2}-148 \sqrt{2} R^{2} m^{4}-5103 \sqrt{2} R^{3} m^{3}-19888 \sqrt{2} R^{4} m^{2}$

$+144 \sqrt{2} R^{2} m^{5}-15340 \sqrt{2} R^{3} m^{4}+62230 \sqrt{2} R^{4} m^{3}+7016 \sqrt{2} R^{5} m^{2}-1632 \sqrt{2} R^{3} m^{5}$

$+47140 \sqrt{2} R^{4} m^{4}-131364 \sqrt{2} R^{5} m^{3}+9616 \sqrt{2} R^{6} m^{2}-12016 \sqrt{2} R^{4} m^{5}+15960 \sqrt{2} R^{5} m^{4}$

$+93432 \sqrt{2} R^{6} m^{3}+1152 \sqrt{2} R^{5} m^{6}-215776 \sqrt{2} R^{6} m^{5}+317072 \sqrt{2} R^{7} m^{4}-59200 \sqrt{2} R^{8} m^{3}$

$-9024 \sqrt{2} R^{7} m^{2}-1152 \sqrt{2} R^{4} m^{6}+102880 \sqrt{2} R^{5} m^{5}-239280 \sqrt{2} R^{6} m^{4}+24608 \sqrt{2} R^{7} m^{3}$

$+42880 \sqrt{2} R^{6} m^{6}+1536 \sqrt{2} R^{8} m^{2}+64192 \sqrt{2} R^{7} m^{5}-54528 \sqrt{2} R^{8} m^{4}+18432 \sqrt{2} R^{9} m^{3}$

$-186752 \sqrt{2} R^{7} m^{6}+257280 \sqrt{2} R^{8} m^{5}-138880 \sqrt{2} R^{9} m^{4}+282880 \sqrt{2} R^{8} m^{6}-207360 \sqrt{2} R^{9} m^{5}$

$+67584 \sqrt{2} R^{10} m^{4}-120576 \sqrt{2} R^{9} m^{6}-61440 \sqrt{2} R^{10} m^{5}-73728 \sqrt{2} R^{10} m^{6}+73728 \sqrt{2} R^{11} m^{5}$

$+55296 \sqrt{2} R^{11} m^{6}-414 \sqrt{2} R m+10777 \mu^{2} R^{2} m^{2}-9268 \mu^{2} R^{2} m^{3}-21502 \mu^{2} R^{3} m^{2}$

$+68136 \mu^{2} R^{3} m^{3}+3588 \mu^{2} R^{4} m^{2}-9135 \mu^{4} R^{2} m^{2}+1152 \mu^{2} R^{2} m^{5}-7832 \mu^{2} R^{3} m^{4}$ 
$+13344 \mu^{2} R^{5} m^{2}+5392 \mu^{4} R^{2} m^{3}+13870 \mu^{4} R^{3} m^{2}-7968 \mu^{2} R^{3} m^{5}+167264 \mu^{2} R^{4} m^{4}$

$+160672 \mu^{2} R^{5} m^{3}-174104 \mu^{2} R^{4} m^{3}-3452 \mu^{2} R^{2} m^{4}-5280 \mu^{2} R^{6} m^{2}+2494 \mu^{4} R^{2} m^{4}$

$-55216 \mu^{4} R^{3} m^{3}-3516 \mu^{4} R^{4} m^{2}+9772 \mu^{6} R^{2} m^{2}+32704 \mu^{2} R^{4} m^{5}-604000 \mu^{2} R^{5} m^{4}$

$-65952 \mu^{2} R^{6} m^{3}-256 \mu^{2} R^{7} m^{2}+576 \mu^{4} R^{2} m^{5}+16676 \mu^{4} R^{3} m^{4}+127376 \mu^{4} R^{4} m^{3}$

$-6080 \mu^{4} R^{5} m^{2}-1920 \mu^{6} R^{2} m^{3}-7856 \mu^{6} R^{3} m^{2}+576 \mu^{2} R^{4} m^{6}+102848 \mu^{2} R^{5} m^{5}$

$+860672 \mu^{2} R^{6} m^{4}+32128 \mu^{2} R^{7} m^{3}+1968 \mu^{4} R^{3} m^{5}-162496 \mu^{4} R^{4} m^{4}-119168 \mu^{4} R^{5} m^{3}$

$+2880 \mu^{4} R^{6} m^{2}+432 \mu^{6} R^{2} m^{4}+59712 \mu^{6} R^{3} m^{3}+2528 \mu^{6} R^{4} m^{2}-5908 \mu^{8} R^{2} m^{2}$

$+24960 \mu^{2} R^{5} m^{6}-846336 \mu^{2} R^{6} m^{5}-587648 \mu^{2} R^{7} m^{4}-9216 \mu^{2} R^{8} m^{3}+15904 \mu^{4} R^{4} m^{5}$

$+481760 \mu^{4} R^{5} m^{4}+39040 \mu^{4} R^{6} m^{3}-3264 \mu^{6} R^{3} m^{4}-78560 \mu^{6} R^{4} m^{3}-768 \mu^{6} R^{5} m^{2}$

$-2592 \mu^{8} R^{2} m^{3}+2304 \mu^{8} R^{3} m^{2}-54784 \mu^{2} R^{6} m^{6}+1710080 \mu^{2} R^{7} m^{5}+202240 \mu^{2} R^{8} m^{4}$

$+4320 \mu^{4} R^{4} m^{6}-220160 \mu^{4} R^{5} m^{5}-609984 \mu^{4} R^{6} m^{4}-7168 \mu^{4} R^{7} m^{3}+576 \mu^{6} R^{3} m^{5}$

$+186752 \mu^{6} R^{4} m^{4}+41088 \mu^{6} R^{5} m^{3}-864 \mu^{8} R^{2} m^{4}-33728 \mu^{8} R^{3} m^{3}-352 \mu^{8} R^{4} m^{2}$

$+2560 \mu^{10} R^{2} m^{2}-285184 \mu^{2} R^{7} m^{6}-1454592 \mu^{2} R^{8} m^{5}-12288 \mu^{2} R^{9} m^{4}-16320 \mu^{4} R^{5} m^{6}$

$+832000 \mu^{4} R^{6} m^{5}+356352 \mu^{4} R^{7} m^{4}+5632 \mu^{6} R^{4} m^{5}-313984 \mu^{6} R^{5} m^{4}-3072 \mu^{6} R^{6} m^{3}$

$-7232 \mu^{8} R^{3} m^{4}+22784 \mu^{8} R^{4} m^{3}+1065984 \mu^{2} R^{8} m^{6}+485376 \mu^{2} R^{9} m^{5}-98560 \mu^{4} R^{6} m^{6}$

$-1202688 \mu^{4} R^{7} m^{5}-109056 \mu^{4} R^{8} m^{4}+282112 \mu^{6} R^{5} m^{5}+186368 \mu^{6} R^{6} m^{4}-2304 \mu^{8} R^{3} m^{5}$

$-106368 \mu^{8} R^{4} m^{4}-10752 \mu^{8} R^{5} m^{3}+14080 \mu^{10} R^{3} m^{3}-480 \mu^{12} R^{2} m^{2}-1185792 \mu^{2} R^{9} m^{6}$

$+548864 \mu^{4} R^{7} m^{6}+827392 \mu^{4} R^{8} m^{5}+16896 \mu^{6} R^{5} m^{6}-574976 \mu^{6} R^{6} m^{5}-4096 \mu^{6} R^{7} m^{4}$

$-8192 \mu^{8} R^{4} m^{5}+88064 \mu^{8} R^{5} m^{4}+442368 \mu^{2} R^{10} m^{6}-819200 \mu^{4} R^{8} m^{6}-282624 \mu^{4} R^{9} m^{5}$

$+154624 \mu^{6} R^{6} m^{6}+376832 \mu^{6} R^{7} m^{5}-2304 \mu^{8} R^{4} m^{6}-176128 \mu^{8} R^{5} m^{5}-51712 \mu^{8} R^{6} m^{4}$

$+43520 \mu^{10} R^{4} m^{4}-2560 \mu^{12} R^{3} m^{3}+589824 \mu^{4} R^{9} m^{6}-395264 \mu^{6} R^{7} m^{6}-1536 \mu^{8} R^{5} m^{6}$

$+153600 \mu^{8} R^{6} m^{5}-221184 \mu^{4} R^{10} m^{6}+294912 \mu^{6} R^{8} m^{6}-119808 \mu^{8} R^{6} m^{6}-102400 \mu^{8} R^{7} m^{5}$

$+71680 \mu^{10} R^{5} m^{5}-7680 \mu^{12} R^{4} m^{4}+98304 \mu^{8} R^{7} m^{6}-73728 \mu^{8} R^{8} m^{6}+$

$49152 \mu^{10} R^{6} m^{6}-12288 \mu^{12} R^{5} m^{5}-8192 \mu^{12} R^{6} m^{6}-6 \sqrt{2} \mu^{2} R+18 \sqrt{2} \mu^{4} R$

$-18 \sqrt{2} \mu^{6} R+6 \sqrt{2} \mu^{8} R+602 \mu^{2} R m-648 \mu^{4} R m+758 \mu^{6} R m-540 \mu^{8} R m$

$+248 \mu^{10} R m-48 \mu^{12} R m-120 \sqrt{2} \mu^{2} m+30 \sqrt{2} \mu^{4} m+6 \sqrt{2} \mu^{6} m$

$+18 \sqrt{2} \mu^{8} m-6 \sqrt{2} \mu^{10} m+282 \sqrt{2} R m^{2}+888 \sqrt{2} R^{2} m+479 \sqrt{2} R m^{3}$

$-834 \sqrt{2} R^{3} m+420 \sqrt{2} R m^{4}+72 \sqrt{2} R^{4} m+600 \sqrt{2} R^{5} m-384 \sqrt{2} R^{6} m$

$+718 \sqrt{2} \mu^{2} R m-320 \sqrt{2} \mu^{4} R m-120 \sqrt{2} \mu^{6} R m+254 \sqrt{2} \mu^{8} R m$

$-118 \sqrt{2} \mu^{10} R m-1010 \sqrt{2} \mu^{2} R m^{2}-1148 \sqrt{2} \mu^{2} R^{2} m-424 \sqrt{2} \mu^{2} R m^{3}$ 
$-30 \sqrt{2} \mu^{2} R^{3} m+420 \sqrt{2} \mu^{2} R m^{4}+988 \sqrt{2} \mu^{2} R^{4} m+760 \sqrt{2} \mu^{2} R^{4} m^{2}$

$+258 \sqrt{2} \mu^{4} R m^{2}+302 \sqrt{2} \mu^{4} R^{2} m-272 \sqrt{2} \mu^{2} R^{5} m-741 \sqrt{2} \mu^{4} R m^{3}$

$+542 \sqrt{2} \mu^{4} R^{3} m+256 \sqrt{2} \mu^{2} R^{6} m-700 \sqrt{2} \mu^{4} R^{4} m-130 \sqrt{2} \mu^{6} R m^{2}$

$-78 \sqrt{2} \mu^{6} R^{2} m-128 \sqrt{2} \mu^{4} R^{5} m-18 \sqrt{2} \mu^{6} R m^{3}-346 \sqrt{2} \mu^{6} R^{3} m$

$+360 \sqrt{2} \mu^{8} R m^{2}+132 \sqrt{2} \mu^{8} R^{2} m+160 \sqrt{2} \mu^{8} R^{3} m-120 \sqrt{2} \mu^{10} R m^{2}$

$+6600 \sqrt{2} \mu^{2} R^{2} m^{2}-1068 \sqrt{2} \mu^{2} R^{2} m^{3}-11780 \sqrt{2} \mu^{2} R^{3} m^{2}-3280 \sqrt{2} \mu^{2} R^{2} m^{4}$

$+23542 \sqrt{2} \mu^{2} R^{3} m^{3}-2978 \sqrt{2} \mu^{4} R^{2} m^{2}+144 \sqrt{2} \mu^{2} R^{2} m^{5}+13636 \sqrt{2} \mu^{2} R^{3} m^{4}$

$-59944 \sqrt{2} \mu^{2} R^{4} m^{3}+13392 \sqrt{2} \mu^{2} R^{5} m^{2}+1250 \sqrt{2} \mu^{4} R^{2} m^{3}+3196 \sqrt{2} \mu^{4} R^{3} m^{2}$

$-6048 \sqrt{2} \mu^{2} R^{3} m^{5}+25976 \sqrt{2} \mu^{2} R^{4} m^{4}+19540 \sqrt{2} \mu^{2} R^{5} m^{3}-7680 \sqrt{2} \mu^{2} R^{6} m^{2}$

$-3552 \sqrt{2} \mu^{4} R^{2} m^{4}-13836 \sqrt{2} \mu^{4} R^{3} m^{3}+2896 \sqrt{2} \mu^{4} R^{4} m^{2}-1132 \sqrt{2} \mu^{6} R^{2} m^{2}$

$+42512 \sqrt{2} \mu^{2} R^{4} m^{5}-168480 \sqrt{2} \mu^{2} R^{5} m^{4}+68384 \sqrt{2} \mu^{2} R^{6} m^{3}+2048 \sqrt{2} \mu^{2} R^{7} m^{2}$

$+4504 \sqrt{2} \mu^{4} R^{3} m^{4}+18488 \sqrt{2} \mu^{4} R^{4} m^{3}-5944 \sqrt{2} \mu^{4} R^{5} m^{2}-2304 \sqrt{2} \mu^{6} R^{2} m^{3}$

$+472 \sqrt{2} \mu^{6} R^{3} m^{2}+1152 \sqrt{2} \mu^{2} R^{4} m^{6}-50336 \sqrt{2} \mu^{2} R^{5} m^{5}+146352 \sqrt{2} \mu^{2} R^{6} m^{4}$

$-47584 \sqrt{2} \mu^{2} R^{7} m^{3}-5136 \sqrt{2} \mu^{4} R^{3} m^{5}-34800 \sqrt{2} \mu^{4} R^{4} m^{4}+1952 \sqrt{2} \mu^{4} R^{5} m^{3}$

$+2304 \sqrt{2} \mu^{4} R^{6} m^{2}-216 \sqrt{2} \mu^{6} R^{2} m^{4}-1620 \sqrt{2} \mu^{6} R^{3} m^{3}-5128 \sqrt{2} \mu^{6} R^{4} m^{2}$

$+2144 \sqrt{2} \mu^{8} R^{2} m^{2}+19200 \sqrt{2} \mu^{2} R^{5} m^{6}-171776 \sqrt{2} \mu^{2} R^{6} m^{5}+120448 \sqrt{2} \mu^{2} R^{7} m^{4}$

$+4096 \sqrt{2} \mu^{2} R^{8} m^{3}+3680 \sqrt{2} \mu^{4} R^{4} m^{5}+51248 \sqrt{2} \mu^{4} R^{5} m^{4}-19648 \sqrt{2} \mu^{4} R^{6} m^{3}$

$-11328 \sqrt{2} \mu^{6} R^{3} m^{4}+416 \sqrt{2} \mu^{6} R^{4} m^{3}+2880 \sqrt{2} \mu^{8} R^{2} m^{3}+1176 \sqrt{2} \mu^{8} R^{3} m^{2}$

$-89216 \sqrt{2} \mu^{2} R^{6} m^{6}+344256 \sqrt{2} \mu^{2} R^{7} m^{5}-126080 \sqrt{2} \mu^{2} R^{8} m^{4}+1152 \sqrt{2} \mu^{4} R^{4} m^{6}$

$-40768 \sqrt{2} \mu^{4} R^{5} m^{5}-16896 \sqrt{2} \mu^{4} R^{6} m^{4}+19456 \sqrt{2} \mu^{4} R^{7} m^{3}-864 \sqrt{2} \mu^{6} R^{3} m^{5}$

$+12432 \sqrt{2} \mu^{6} R^{4} m^{4}-28128 \sqrt{2} \mu^{6} R^{5} m^{3}+9024 \sqrt{2} \mu^{8} R^{3} m^{3}+1280 \sqrt{2} \mu^{8} R^{4} m^{2}$

$-920 \sqrt{2} \mu^{10} R^{2} m^{2}+36864 \sqrt{2} \mu^{2} R^{7} m^{6}-28160 \sqrt{2} \mu^{2} R^{8} m^{5}-12672 \sqrt{2} \mu^{4} R^{5} m^{6}$

$+64768 \sqrt{2} \mu^{4} R^{6} m^{5}-18048 \sqrt{2} \mu^{4} R^{7} m^{4}-20992 \sqrt{2} \mu^{6} R^{4} m^{5}-21120 \sqrt{2} \mu^{6} R^{5} m^{4}$

$+11520 \sqrt{2} \mu^{8} R^{3} m^{4}+5376 \sqrt{2} \mu^{8} R^{4} m^{3}-960 \sqrt{2} \mu^{10} R^{2} m^{3}+191744 \sqrt{2} \mu^{2} R^{8} m^{6}$

$-127488 \sqrt{2} \mu^{2} R^{9} m^{5}-7936 \sqrt{2} \mu^{4} R^{6} m^{6}-33792 \sqrt{2} \mu^{4} R^{7} m^{5}+53248 \sqrt{2} \mu^{4} R^{8} m^{4}$

$-1152 \sqrt{2} \mu^{6} R^{4} m^{6}+41024 \sqrt{2} \mu^{6} R^{5} m^{5}-66688 \sqrt{2} \mu^{6} R^{6} m^{4}+18944 \sqrt{2} \mu^{8} R^{4} m^{4}$

$+5120 \sqrt{2} \mu^{8} R^{5} m^{3}-3520 \sqrt{2} \mu^{10} R^{3} m^{3}-178176 \sqrt{2} \mu^{2} R^{9} m^{6}+39680 \sqrt{2} \mu^{4} R^{7} m^{6}$

$+25600 \sqrt{2} \mu^{4} R^{8} m^{5}-10752 \sqrt{2} \mu^{6} R^{5} m^{6}-78848 \sqrt{2} \mu^{6} R^{6} m^{5}+23040 \sqrt{2} \mu^{8} R^{4} m^{5}$ 


$$
\begin{aligned}
& +13056 \sqrt{2} \mu^{8} R^{5} m^{4}-3840 \sqrt{2} \mu^{10} R^{3} m^{4}+18432 \sqrt{2} \mu^{2} R^{10} m^{6}-24576 \sqrt{2} \mu^{4} R^{8} m^{6} \\
& +49152 \sqrt{2} \mu^{4} R^{9} m^{5}+28416 \sqrt{2} \mu^{6} R^{6} m^{6}-53248 \sqrt{2} \mu^{6} R^{7} m^{5}+15872 \sqrt{2} \mu^{8} R^{5} m^{5} \\
& +10240 \sqrt{2} \mu^{8} R^{6} m^{4}-6400 \sqrt{2} \mu^{10} R^{4} m^{4}+36864 \sqrt{2} \mu^{4} R^{9} m^{6}-77824 \sqrt{2} \mu^{6} R^{7} m^{6} \\
& +18432 \sqrt{2} \mu^{8} R^{5} m^{6}+15360 \sqrt{2} \mu^{8} R^{6} m^{5}-7680 \sqrt{2} \mu^{10} R^{4} m^{5}+12288 \sqrt{2} \mu^{6} R^{8} m^{6} \\
& +8192 \sqrt{2} \mu^{8} R^{7} m^{5}-3584 \sqrt{2} \mu^{10} R^{5} m^{5}+6144 \sqrt{2} \mu^{8} R^{7} m^{6}-6144 \sqrt{2} \mu^{10} R^{5} m^{6} \\
& \left.+2048 \sqrt{2} \mu^{10} R^{6} m^{6}\right) .
\end{aligned}
$$

Cuando tomamos $m \rightarrow 0$

$$
\begin{aligned}
p(\mu)= & \frac{1}{1024(R-1)^{9}} \mu^{2} \cdot(\mu+1) \cdot(\mu-1) \cdot\left(\left(2 \sqrt{2}+\frac{5}{2}\right) \mu^{2}-2 \sqrt{2}-R(4 \sqrt{2}+5)-\frac{5}{2}\right) . \\
& \left(\mu^{4}+(3 \sqrt{2}-2) \mu^{2}+(-4 \sqrt{2}-5) R^{2}+(8-3 \sqrt{2}) R+3 \sqrt{2}+1\right) . \\
& \left(\left(\frac{2 \sqrt{2}}{7}-\frac{5}{14}\right) \mu^{2}-\frac{2 \sqrt{2}}{7}+R\left(\frac{4 \sqrt{2}}{7}-\frac{5}{7}\right)+\frac{5}{14}\right) .
\end{aligned}
$$

El factor $(\mu-1)$ nos muestra una raíz real positiva, suficiente para concluir que las órbitas generadas son inestables.

OBSERVACIÓN 3 Como hemos visto en los equilibrios relativos del problema de dos cuerpos en $S^{2}$ y en los equilibrios relativos elípticos en $H^{2}$ la estabilidad se comporta muy diferente dependiendo del espacio fase que tomemos en cuenta, esto es algo que no sucede en los equilibrios relativos hiperbólicos ya que tenemos el mismo resultado independientemente del espacio fase en el que se exponga el problema.

De forma análoga a como lo hemos estado haciendo primero consideramos el espacio fase

$$
\left(\mathbb{R}^{2 n} \backslash \triangle\right) \times T \mathbb{R}^{2 n} \subseteq \mathbb{R}^{8},
$$

donde $T \mathbb{R}^{2 n}$ es el haz tangente de $\mathbb{R}^{2 n},(n=2)$.

Por otra parte si consideramos tres partículas, en donde una de ellas de masa $m$ en el punto $(0,0,1)$, el problema está planteado en el espacio fase

$$
\left(\mathbb{R}^{2 n} \backslash \triangle\right) \times T \mathbb{R}^{2 n} \subseteq \mathbb{R}^{12},
$$


$y T \mathbb{R}^{2 n}$ es el haz tangente de $\mathbb{R}^{2 n},(n=3)$.

En ambos casos $\triangle$ está dado por las correspondientes singularidades, explicadas en la sección (2.5). 


\section{Capítulo 7 Conclusiones}

Terminaremos nuestro trabajo de tesis resumiendo nuestros resultados en este capítulo. Como vimos el planteamiento del problema de estabilidad de equilibrios relativos del problema curvado de los $n$-cuerpos no es tan complicado, sin embargo su análisis se dificulta ya que se analizan matrices y sus respectivos polinomios característicos que están expresados en la variable $\mu$ (grado 12) y cuyos coeficientes son funciones racionales dependientes de las variables de las masas, $m, M$ (grado 6) y la posición de las partículas, $z$ (grado 9). A pesar de la dificultad logramos obtener las siguientes conclusiones, recordando que los resultados son referentes a estabilidad de tipo espectral.

\section{- Problema de dos y tres cuerpos en $S^{2}$}

(a) Consideremos órbitas periódicas del problema curvado de dos cuerpos en $S^{2}$. Si los cuerpos con masas $m_{1}=m_{2}$ se encuentran fijas en los lados opuestos de un círulo que gira uniformemente en un plano paralelo al ecuador $z=0$, entonces para $z \in(-1,1)$ las órbitas generadas son estables si $1>|z| \geq \frac{1}{\sqrt{2}}$ e inestables si $0<|z|<\frac{1}{\sqrt{2}}$.

(b) Consideremos órbitas eulerianas periódicas del problema curvado de tres cuerpos en $S^{2}$ con masas $m_{1}=m_{2}$ fijas en los lados opuestos de un círculo que rota uniformemente en un plano paralelo al ecuador $z=0$, y la partícula $m_{3}$ de masa despreciable fija en el polo norte $(0,0,1)$, entonces las órbitas generadas son inestables para $z>0$, y además este tipo de órbitas no existen si $0>z>-1$.

(c) Consideremos órbitas eulerianas periódicas del problema curvado de tres cuerpos en $S^{2}$, con masas $m_{1}=m_{2}=m_{3}$, en donde $m_{1}$ y $m_{2}$ se encuentran fijas en los lados opuestos de un círculo que rota uniformemente en un plano paralelo al ecuador $z=0$, y la partícula $m_{3}$ fija en el polo norte $(0,0,1)$, entonces para $z \in(-1 / 2,0) \cup(0,1)$ las órbitas generadas son inestables, y el movimiento no es posible si $z \in(-1,-1 / 2)$.

(d) Consideremos órbitas eulerianas del problema curvado de tres cuerpos en $S^{2}$, con dos masas despreciables fijas en los lados opuestos de un círculo que rota uniformemente en un plano paralelo al ecuador $z=0$, y una partícula con masa $m$ fija en el polo norte $(0,0,1)$, entonces para $z \in(0,1)$ las órbitas generadas son estables. 


\section{- Problema de dos y tres cuerpos en $H^{2}$ Equilibrios relativos elípticos}

(e) Consideremos órbitas periódicas del problema curvado de dos cuerpos en $H^{2}$. Si los cuerpos con masas $m_{1}=m_{2}$ se encuentran fijos en los lados opuestos de un círulo que gira uniformemente en un plano paralelo al plano $x y$, entonces para $z \in(1, \infty)$ las órbitas generadas son estables si $1<z<\frac{\sqrt{3}}{\sqrt{2}}$ e inestables si $z>\frac{\sqrt{3}}{\sqrt{2}}$.

(f) Consideremos órbitas eulerianas del problema curvado de tres cuerpos en $H^{2}$, con masas $m_{1}=m_{2}$ fijas en los lados opuestos de un círculo que gira uniformemete un un plano paralelo al plano $x y$ a altura $z$, y la partícula $m_{3}$ con masa despreciable fija en el punto $(0,0,1)$, entonces para $z \in(1, \infty)$ las órbitas generadas son inestables.

(g) Consideremos órbitas eulerianas periódicas del problema curvado de tres cuerpos en $H^{2}$, con masas $m_{1}=m_{2}=m_{3}$, en donde $m_{1}$ y $m_{2}$ se encuentran fijas en los lados opuestos de un círculo que rota uniformemente y de forma paralela al plano $x y$, y la partícula $m_{3}$ fija en el punto $(0,0,1)$, entonces para cualquier $z>1$ las órbitas generadas son inestables.

(h) Consideremos órbitas eulerianas elípticas del problema curvado de tres cuerpos en $H^{2}$, con dos masas despreciables fijas en los lados opuestos de un círculo que rota uniformemente en un plano paralelo al plano $x y$, y una partícula de masa $m=1$ fija en el punto $(0,0,1)$, entonces para $z \in(1, \infty)$ las órbitas generadas son estables.

\section{Equilibrios relativos hiperbólicos}

(i) Consideremos órbitas eulerianas del problema curvado de los dos cuerpos con masas iguales en $H^{2}$, donde el cuerpo $m_{i}$ tiene coordenadas $q_{i}=\left(x_{i}, y_{i}, z_{i}\right)$. Si las masas $m_{1}$ y $m_{2}$ se mueven a lo largo de hipérbolas de la forma $x_{1}=c t e, x_{2}=$ $-x_{1}, y_{1}=y_{2}=r \sinh (t), z_{1}=z_{2}=r \cosh (t), t \in \mathbb{R}$, entonces las órbitas generadas son inestables para cualquier $r>1$.

(j) Consideremos órbitas eulerianas del problema curvado de los tres cuerpos en $H^{2}$, donde el cuerpo $m_{i}$ tiene coordenadas $q_{i}=\left(x_{i}, y_{i}, z_{i}\right)$. Si las masas $m_{1}$ y $m_{2}$ se mueven a lo largo de hipérbolas de la forma $x_{1}=$ cte, $x_{2}=-x_{1}, y_{1}=y_{2}=$ $r \sinh (t), z_{1}=z_{2}=r \cosh (t), t \in \mathbb{R}$, y la partícula de $m_{3}$ de masa despreciable se encuentra moviéndose sobre la hipérbola que pasa por $(0,0,1)$, entonces las órbitas generadas son inestables para cualquier $r>1$.

\section{Perspectivas}

El estudio de los $n$-cuerpos en espacios curvados es muy reciente por lo que aún existen muchos caminos por explorar, como mencionamos en la introducción se han hecho estudios sobre movimientos de tipo lagrangiano, que junto con nuestro trabajo dan una idea más clara de lo que sucede con ciertos tipos de movimientos del problema de tres cuerpos. Como trabajo posterior a éste se tiene como proyecto analizar el problema de los tres cuerpos en espacios 
curvados de manera un poco más general, observando colisiones, escapes o valores de energía por ejemplo. Como sabemos, el problema newtoniano de tres cuerpos es un problema bastante complicado, de éste se conocen sólo las soluciones homográficas, para poder entender mejor este problema usualmente se trabaja en modelos donde se imponen ciertas simetrías. Debido a la complejidad que se presenta en espacios curvados hemos considerado estudiar la dinámica del problema en $S^{2}$ y en $H^{2}$ también tomando en cuenta algunas simetrías sobre masas y posiciones de las partículas. Un ejemplo inmediato de este tipo de problemas es el llamado problema isósceles, que consiste en estudiar la dinámica de tres partículas que forman en todo tiempo una figura de triángulo isósceles.

A pesar de las dificultades que se tienen en el problema de los tres cuerpos se han hecho algunos trabajos sobre ciertos tipos de movimientos del problema de los 4 cuerpos en espacios curvados como en [8], y en lo que también podría continuar este trabajo es en analizar también algún otro tipo de movimiento generado por el problema de los 4 cuerpos en $H^{2}$.

Debido a que el estudio de este tipo de problemas es muy reciente el camino a seguir es muy variado. 


\section{Apéndice}

En esta sección mostraremos algunas ideas básicas del modelo propuesto por Weierstrass del plano hiperbólico. Este modelo es el que se ha utilizado para mostrar resultados del problema de $n$ cuerpos en espacios de curvatura constante negativa.

El modelo de Weierstrass esta construideo a partir de una de las hojas del hiperboloide dado por la ecuación $x^{2}+y^{2}-z^{2}=-1$ en el espacio de Minkowski de dimensión $3, \mathbb{M}^{3}=\left(\mathbb{R}^{3}, \odot\right)$, en donde $(\odot)$ representa el producto interno de Lorentz, definido como $a \odot b=a_{x} b_{x}+a_{y} b_{y}-a_{z} b_{z}$, con $a=\left(a_{x}, a_{y}, a_{z}\right)$ y $b=\left(b_{x}, b_{y}, b_{z}\right)$. Escogemos la hoja del hiperboloide, identificado con el plano de Bolyai-Lovachevski, denotado por $H^{2}$.

Una transformación $T: \mathbb{M}^{3} \rightarrow \mathbb{M}^{3}$ es ortogonal si $T(a) \odot T(a)=a \odot a$ para cualquier $a \in \mathbb{M}^{3}$. El grupo de transformaciones ortogonales junto con el producto interior de Lorentz forma el grupo ortogonal $O\left(\mathbb{M}^{3}\right)$, dado por las matrices de determinante 1 y -1 . Entonces el grupo $S O\left(\mathbb{M}^{3}\right)$ de transformaciones de determinante 1 es un subgrupo de $O\left(\mathbb{M}^{3}\right)$. Otro subgrupo de $O\left(\mathbb{M}^{3}\right)$ es $G\left(\mathbb{M}^{3}\right)$, formado por las transformaciones $T$ que dejan a $H^{2}$ invariante. De hecho $G\left(\mathbb{M}^{3}\right)$ contiene al subgrupo Lor $=G\left(\mathbb{M}^{3}\right) \cap S O\left(\mathbb{M}^{3}\right)$.

Definamos las rotaciones de Lorentz alrededor de un eje como los subgrupos uniparamétricos de $\operatorname{Lor}\left(\mathbb{M}^{3}\right)$ que dejan los ejes fijos. El teorema del eje principal nos dice que todas las transformaciones de Lorentz tienen una de las siguientes formas [15]

$$
\begin{aligned}
& A=P\left(\begin{array}{ccc}
\cos \theta & -\sin \theta & 0 \\
\sin \theta & \cos \theta & 0 \\
0 & 0 & 1
\end{array}\right) P^{-1}, \\
& B=P\left(\begin{array}{ccc}
1 & 0 & 0 \\
0 & \cosh s & \sinh s \\
0 & \sinh s & \cosh s
\end{array}\right) P^{-1}
\end{aligned}
$$

$\mathrm{O}$

$$
C=P\left(\begin{array}{ccc}
1 & -t & t \\
t & 1-t^{2} / 2 & t^{2} / 2 \\
t & -t^{2} / 2 & 1+t^{2} / 2
\end{array}\right) P^{-1}
$$


donde $\theta \in[0,2 \pi), s, t \in \mathbb{R}$, y $P \in \operatorname{Lor}\left(\mathbb{M}^{3}\right)$. Éstas son llamadas transformaciones elípticas, hiperbólicas y parabólicas, respectivamente. Las transformaciones elípticas son rotaciones alrededor del eje $z$; las transformaciones hiperbólicas son rotaciones alrededor del eje $x$; y las transformaciones parabólicas son rotaciones alrededor de la linea $y=z, x=0$.

Cabe mencionar que geodésicas en $H^{2}$ son hipérbolas que se obtienen con la intersección del hiperboloide y planos que pasan por el origen del sistema coordenado. Para cualquiera dos puntos distintos $a$ y $b$ de $H^{2}$ pasa una única geodésica que los une, y la distancia entre esos puntos está dada por $d(a, b)=\cosh ^{-1}(-a \odot b)$.

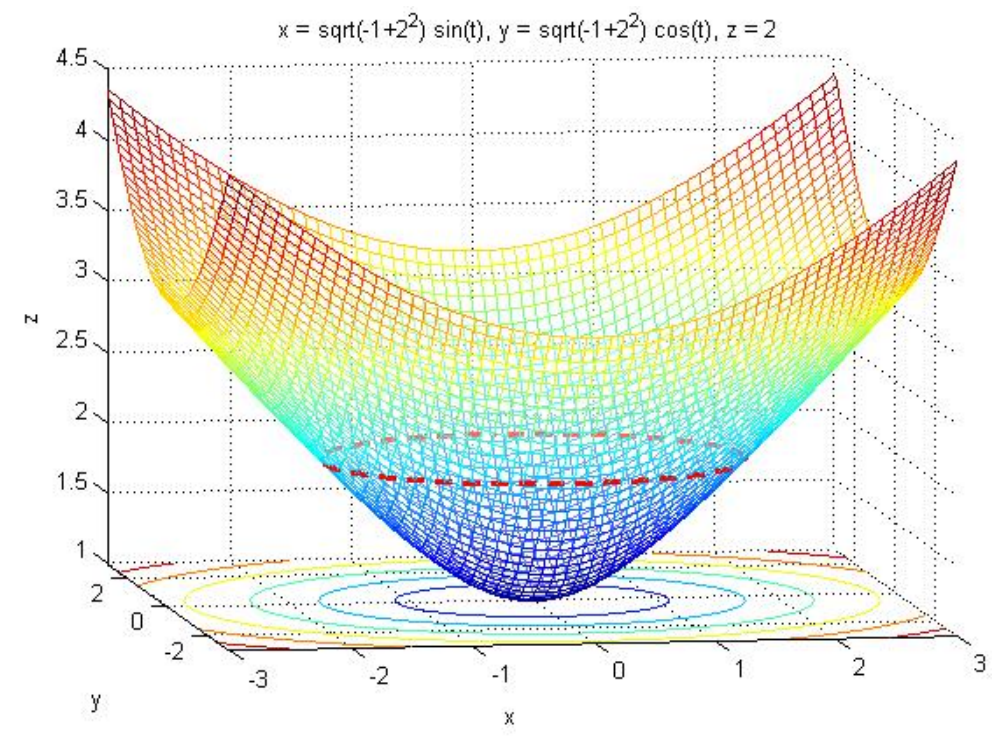

Figura 1: La linea punteada marca la trayectoria del punto $(\sqrt{3}, 0,2)$ al aplicarle la transformación $A$. 


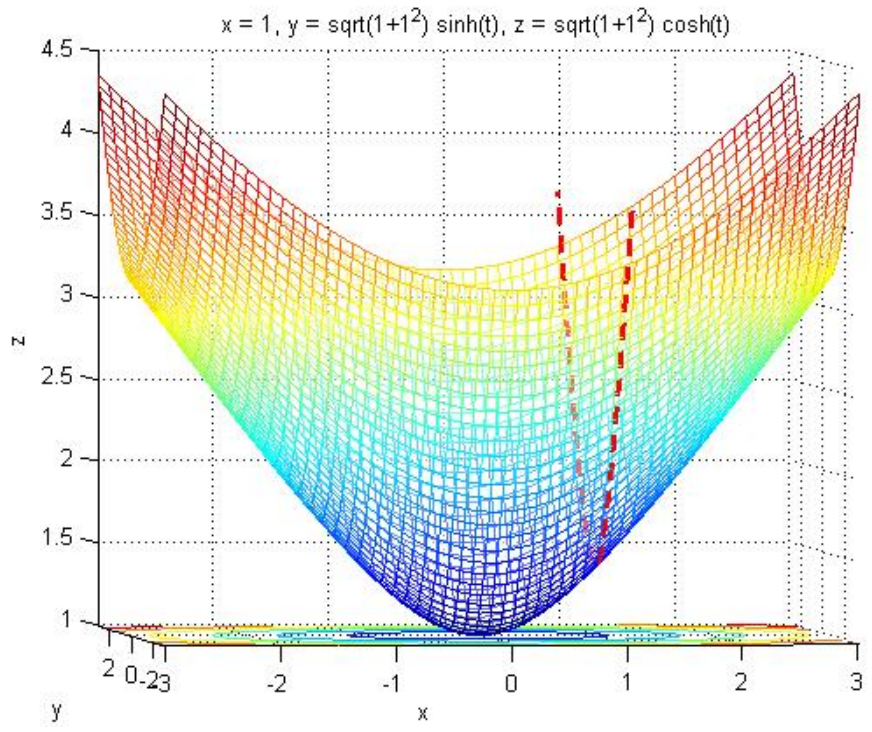

Figura 2: La linea punteada marca la trayectoria del punto $(1,0, \sqrt{2})$ al aplicarle la transformación $B$.

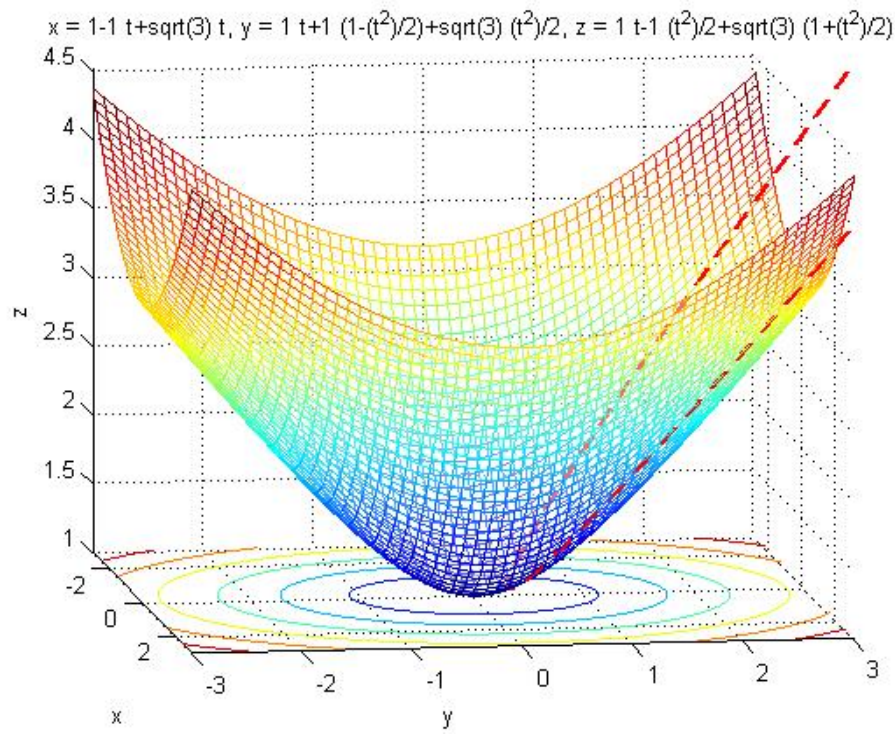

Figura 3: La linea punteada marca la trayectoria del punto $(1,1,2)$ al aplicarle la transformación $C$. 


\section{Bibliografía}

[1] ARNOLD, V. I., KOSLOV,V., AND NEISHTADT, A. Mathematical Aspects of Classical and Celestial Mechanics. Springer-Verlag. Berlin, Heidelberg, New York. (1997).

[2] BOLYAI, J., AND BOLYAI. W. Geometrische Untersuchungen. Hrsg. P. Stäckel, Teubner, Leipzig-Berlin. 1913.

[3] BRUnS, H. Über die Integrale des VielKörper- Problems. Acta Mathematica. 11. (1887).

[4] CARIÑENA, J.F., RAÑADA, M.F., AND SANTANDER, M. Central Potentials on Spaces of Constant Curvature: The Kepler Problem on the Two Dimensional Sphere $S^{2}$ and the Hyperbolic Plane $H^{2}$. Journal of Mathematical Physics. 46, 052702 (2005).

[5] DIACU, F. On the Singularities of the Curved n-Body Problem. Transactions of the American Mathematical Society. Vol 363 (2249-2264). (2011)

[6] DIACU, F. Polygonal Homographic Orbits of the Curved n-Body Problem. Transactions of the American Mathematical Society. Vol 364 (2783-2802). (2012)

[7] DIACU, F. Relative Equilibria of the Curved n-Body Problem. Atlantis Series in Dynamical Systems, 2012

[8] DiACU, F., MARTÍNEZ, R., PÉREZ-CHAVELA, E., AND SIMÓ, CARLES. On the Stability of Tetrahedral Relative Equilibria in the Positively Curved 4-Body Problem. Physica D. Vol 256-257 (21-35). (2013).

[9] DIACU, F., PÉREZ-CHAVElA, E., AND REYES-VICTORIA, G. An Intrinsic Approach in the Curved n-Body Problem. The Negative Curvature Case. Journal of Differential Equations. Vol 252 (4529-4562). (2012).

[10] DiACU, F., PÉREZ-CHAVELA, E., AND SANTOPRETE, M. The n-Body Problem in Spaces of Constant Curvature. Part I: Relative Equilibria. Journal of Nonlinear Science. 22:(247-266). (2012).

[11] DiaCU, F., PÉREZ-ChaVEla, E., AND SANTOPRETE, M. The n-Body Problem in Spaces of Constant Curvature. Part II: Singularities. Journal of Nonlinear Science. 22:(267-275). (2012). 
[12] DO CARMO, M. Differential Geometry of Curves and Surfaces. Prentice Hall, New Jersey USA, 1976.

[13] DUBrovin, B.A., FOMENKO, A.T., AND NOVIKOV S.P. Modern Geometry- Methods and Applications. Springer-Verlag. New York. Second Edition. 1992.

[14] EULER, L. De Moto Rectilineo Trium Corporum Se Mutuo Attahentium. Novi Comm. Acad. Sci. Imp. Potrop. 11, 144-151. (1767).

[15] HANO, J., NOMIZU, K., On Isometric Immersions of the Hyperbolic Plane Into the Lorent-Minkowski Space and the Monge-Ampere Equation of Certain Type. Mathematische Annalen. 262, 245-253 (1983).

[16] HSIUNG, C., A first Course in Differential Geometry. A Wiley Interscience Publication, USA, 1981.

[17] JUlliaRD-TOSEL, E. Bruns's Theorem: The Proof and Some Generalizations. Celestial Mechanics and Dynamical Astronomy. 76: 241-281. (2000).

[18] KILlinG, A. A., Die Mechanik in den Nichteuklidischen Raumformen. Journal für die Reine und Angewandte Mathematik. 89, 265-287. (1880).

[19] KOZLOV, V.V., AND HARIN, A.O. Kepler's Problem in Constant Curvature Spaces. Celestial Mechanics and Dynamical Astronomy. 54, 393-399. (1992).

[20] LAGRAnGE, J. Essai Sur Le Probleme des Trois Corps. Euvres, vol. 6, Paris, 272292. (1772).

[21] LIEBMANN, H. Die Kegelschnitte und die Planetenbewegung im Nichteuklidischen Raum. Berichte Königlich-Sächsischen Gesellschaft der Wissenschaften, Math. Phys. Klasse. 54, 393-423. (1902).

[22] LIPSCHITZ, R. Extension of the Planet-Problem to a Space of $n$ Dimensions and Constant Integral Curvature. The Quarterly Journal of Pure and Applied Mathematics. 12, 349-370. (1873).

[23] LOVACHEVSKI, N. I. The New Foundations of Geometry with Full Theory of Parallels (in Russian). 1835-1838. In Collected Works, V. 2, GITTL, Moscow. 159. 1949.

[24] MARCHAL, C. The Three Body Problem. Elsevier. 1990.

[25] MARTÍNEZ, R., AND SIMÓ, C. On the Stability of the Lagrangian Homographic Solutions in a Curved Three-Body Problem. Discrete and Continuous Dynamical Systems. Vol 33 (1157-1175). (2013)

[26] MEYER, K., AND HALL, G. Introduction to Hamiltonian Dynamical Systems and the N-Body Problem. Springer-Verlag. New York. 1992. 
[27] MOUlTON, F.R. An Introduction to Celestial Mechanics. Dover. 1970.

[28] NEWTON, I. Philosophiae Naturalis Principia Mathematica. University of California Press, Motte's translation. 1934.

[29] PEREZ-CHAVELA, E., Sobre la Profesión más Antigua del mundo. Miscelanea Matemática, Sociedad Matemática Mexicana, 46. (2005).

[30] PEREZ-CHAVELA, E., AND REYES-VICTORIA, G., An Intrinsic Approach in the Curved n-Body Problem. The Positive Curvature Case. Transactions of the American Mathematical Society, Vol 364 (3805-3827). (2012)

[31] POINCARE, H. Les Méthodes Nouvelles de la Mécanique Celeste. English Translation. History of Modern Physics and Astronomy 13, The American Institute of Physics. (1993).

[32] ROY, A. Orbital Motion. Adam Hilger, Bristol. (1968).

[33] SABADELL, M.A., Astronomía: Una Historia de Esperanzas y Temores. Mediatika. Cuadernos de Medios de Comunicación (573-581). (2002).

[34] SCHROEDINGER, E. A Method for Determining Quantum-Mechanical Eigenvalues and Eigenfunctions. Proceedings of the Royal Irish Academy, Sect. A 46, (2008), 042903,16 pp.

[35] SERRET, P.J. Théorie Nouvelle Géometrique et Mecanique des Lignes a Double Courbure. Librave de Mallet-Bachelier, Paris. 1860.

[36] SIMÓ, C. El Problema de n Cuerpos. Revistes Catalanes amb Accés Obert. Versión en linea http://www.raco.cat/index.php/PublicacionsSeccioMatematiques/ article/view/37148/37022

[37] SOMASUndARAM, D. Differential Geometry: A First Course. Alpha Science International, Ltd. 2004.

[38] SZEBEHELY, V. Theory of Orbits. Academic Press. London. 1967. 


\section{Índice alfabético}

Ecuaciones de Movimiento Espacios Curvos,

10

Energía, 13

Equilibrio Relativo, 21

Elíptico, 22, 33

Euleriano, 22

Hiperbólico, 33

Lagrangiano, 28, 33

Parabólico, 34

Estabilidad Espectral, 43

Forma Hamiltoniana, 12

Lorentz, 133

Minkowski, 133

Momento Angular, 13

Potencial Cotangente, 7

Problema Restringido, 52

Punto Fijo, 22

Singularidades, 14

Superficie, 5

Curvatura Gaussiana, 6

Curvatura Media, 6

Curvatura Principal, 5

Trigonometría Superficies Curvas, 6

Weierstrass, 133 


\title{
ESTABILIDAD DE EQUILIBRIOS RELATIVOS EN ESPACIOS CURVADOS
}

\author{
Tesis que presenta \\ Juan Manuel Sánchez Cerritos \\ Para obtener el grado de \\ Maestro en Ciencias Matemáticas
}

Dirigida por: DR. ERNESTO PÉREZ̨ CHAVELA

Sinodales:

DR. FLORIN DIACU,

DR. ERNESTO PÉREZ CHAVELA,

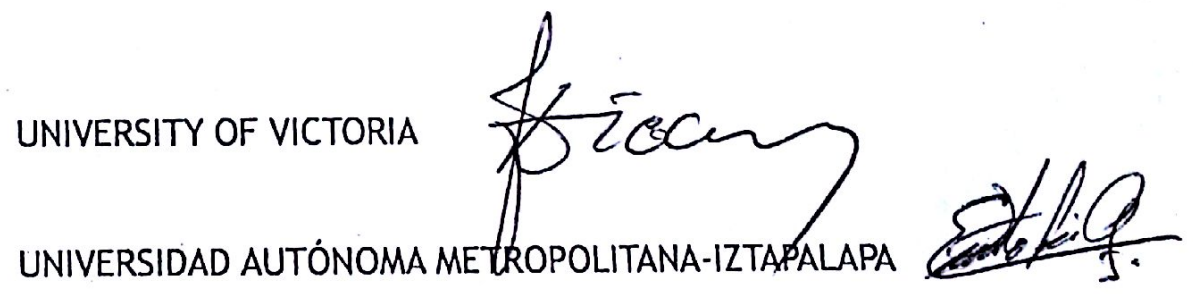

DR. EDUARDO PIÑA GARZA,

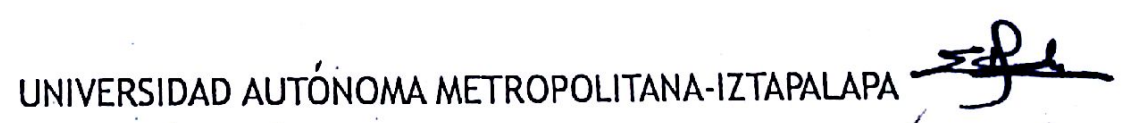

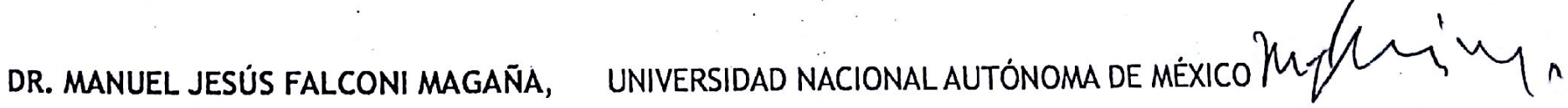

México DF., Diciembre 2013 